\title{
Density Functional Theory Simulations of Rare-Earth Hexaborides: Bulk and Surface Studies
}

\author{
Dissertation \\ zur Erlangung des mathematisch-naturwissenschaftlichen Doktorgrades \\ "Doctor rerum naturalium" \\ der Georg-August-Universität Göttingen
}

im Promotionsprogramm ProPhys

der Georg-August University School of Science (GAUSS)

vorgelegt von

Florian Sohn

aus Siegburg

Göttingen, 2021 


\section{Betreuungsausschuss}

Prof. Dr. Peter E. Blöchl

Institut für Theoretische Physik

Technische Universität Clausthal

PD Dr. Martin Wenderoth

IV. Physikalisches Institut

Georg-August-Universität Göttingen

PD Dr. Salvatore R. Manmana

Institut für Theoretische Physik

Georg-August-Universität Göttingen

\section{Mitglieder der Prüfungskommission}

Referent: Prof. Dr. Peter E. Blöchl

Institut für Theoretische Physik

Technische Universität Clausthal

Korreferent: Prof. Dr. Stefan Kehrein

Institut für Theoretische Physik

Georg-August-Universität Göttingen

\section{Weitere Mitglieder der Prüfungskommission}

PD Dr. Martin Wenderoth

IV. Physikalisches Institut

Georg-August-Universität Göttingen

PD Dr. Salvatore R. Manmana

Institut für Theoretische Physik

Georg-August-Universität Göttingen

apl. Prof. Dr. Vasile Moshnyaga

I. Physikalisches Institut

Georg-August-Universität Göttingen

Prof. Dr. Marcus Müller

Institut für Theoretische Physik

Georg-August-Universität Göttingen

Tag der mündlichen Prüfung: 25. Oktober 2021 


\section{Contents}

1 Introduction 1

2 Rare-earth hexaborides $\quad 5$

2.1 Rare earths .................. 5

2.2 Boron ......................... 5

2.3 The rare-earth hexaborides . . . . . . . . . 6

2.4 The $\mathrm{LaB}_{6}$ surface $\ldots \ldots \ldots \ldots$

3 Methods $\quad 11$

3.1 Electronic correlations . . . . . . . . . . . . . 11

3.2 Many-particle physics . . . . . . . . . . . . . . . 13

3.3 The Born-Oppenheimer approximation . . . . . . . . . . 14

3.4 The electronic Hamiltonian . . . . . . . . . . . . . . . 16

3.5 The Anderson impurity model . . . . . . . . . . . . . . . . . 18

3.6 The Rayleigh-Ritz principle . . . . . . . . . . . . . . . . . . . 19

3.7 Hartree-Fock theory . . . . . . . . . . . . . . . . . . . . . . 19

3.7.1 The Hartree-Fock approximation of the Anderson impurity model ...................... 26

3.8 Density-functional theory . . . . . . . . . . . . 28

3.8.1 The Hohenberg-Kohn-theorem . . . . . . . . . . . 28

3.8.2 Levy's constrained search functional . . . . . . . . 30

3.8.3 Lieb's ensemble state density functional . . . . . . . . 31

3.8.4 The adiabatic connection of non-interacting and interacting system .................. 32

3.8.5 Spin-polarized systems . . . . . . . . . . . . . 34

3.8.6 The Kohn-Sham equations . . . . . . . . . . . . 35

3.9 The exchange-correlation functional . . . . . . . . . . 37

3.9.1 The local density approximation . . . . . . . . 38

3.9.2 The local spin density approximation . . . . . . . 38

3.9.3 The generalized gradient approximation . . . . . . 39

3.9.4 Extensions to the LSDA and GGA . . . . . . . . 40

3.9.5 Hybrid functionals . . . . . . . . . . . . 41

3.9.6 The PBE0r hybrid functional . . . . . . . . . . . . . 43

3.10 Car-Parrinello dynamics . . . . . . . . . . . . . 45

3.11 The projector augmented-wave method . . . . . . . . . . 47

3.11 .1 Basic formalism . . . . . . . . . . . . 47

3.11.2 Expectation values, the density, and the total energy . 49

3.12 The density of states . . . . . . . . . . . . . . 51

3.13 Scanning tunneling microscopy . . . . . . . . . . . 55

3.13 .1 Tunneling theory . . . . . . . . . . . 57

3.13.2 Theory of the STM from perturbation theory . . . . 57 
3.13.3 The tunneling current . . . . . . . . . . . . 60

3.13 .4 The tunnel matrix . . . . . . . . . . . . . 61

3.13.5 The Tersoff-Hamann approximation . . . . . . . 62

3.13.6 Scanning tunneling spectroscopy . . . . . . . . . 65

4 DFT bulk simulations of rare-earth hexaborides 67

4.1 The electronic structure of $\mathrm{a}_{6}$ octahedron . . . . . . . . 67

4.1.1 Exploiting symmetries to diagonalize the Hamiltonian 68

4.1.2 Symmetrized orbitals of the $\mathrm{B}_{6}$ octahedra . . . . . . 69

4.2 Bulk simulation of the boron network . . . . . . . . 69

4.3 DFT bulk simulations of lanthanum hexaboride . . . . . . 73

4.3.1 Results with the PBE functional . . . . . . . . 73

4.3.2 Results with the PBE0r hybrid functional . . . . . 76

4.3.3 Discussion . . . . . . . . . . . . . 77

4.4 Light rare-earth hexaborides . . . . . . . . . . . . . . . . 78

4.4.1 Cerium hexaboride . . . . . . . . . . . 78

4.4.2 Praseodymium hexaboride . . . . . . . . . . 81

4.4.3 Neodymium hexaboride . . . . . . . . . . . 85

4.4.4 Dependence of the $f$-state energies on the exchange mixing factor ................. 88

4.4.5 $f$-state occupancy at an exchange mixing factor of 0.1591

4.5 Discussion . . . . . . . . . . . . . . . 91

5 The density of states of localized orbitals with fractional occupancy: study of a model system $\quad 95$

5.1 The model system . . . . . . . . . . . . . . . . . . . . 97

5.1 .1 The bath . . . . . . . . . . . . 97

5.1 .2 The impurity . . . . . . . . . . . . . 98

5.2 Many-body approach to the model . . . . . . . . . . . . 99

5.2.1 The ground state total energy of the model . . . . . . 101

5.2.2 The spectral function . . . . . . . . . . . . . . 104

5.3 Hartree-Fock approach to the model system . . . . . . . . . 111

5.3.1 The ground state total energy . . . . . . . . . . . 113

5.3.2 The spectral function in the Hartree-Fock approximation 116

5.4 Discussion of the spinless model . . . . . . . . . . . . 122

5.5 Spin-resolved model system with Hund's coupling . . . . . . . 124

5.6 Many-body approach to the spin-resolved model . . . . . . . . 126

5.6.1 The ground state total energy in the many-body approach 126

5.6.2 The impurity spectral function in the many-body approach . . . . . . . . . . . . . . 128

5.7 Hartree-Fock approach to the spin-resolved model . . . . . . . 131

5.7.1 The ground state total energy of the spin-resolved model in the Hartree-Fock approximation . . . . . . . . . . . 131

5.7.2 The ground state ensemble of the impurity in the HartreeFock approximation . . . . . . . . . . . . . . 134

5.7.3 The impurity spectral function with respect to $\hat{H}_{\text {loc }}^{\mathrm{HF}} \cdots 134$

5.7.4 The impurity spectral function with respect to $\hat{H}_{\text {loc }}$. . 136 
5.8 Discussion of the spin-resolved model . . . . . . . . . . . 139

5.9 Comparison of the spectral function of the model to the DFT

DOSs of the light rare-earth hexaborides . . . . . . . . . 143

5.9.1 Spectral function of the spinless model versus the DFT

DOSs of the light rare-earth hexaborides . . . . . . . 143

5.9.2 Including a model for the double counting . . . . . . . 147

5.10 Summary . . . . . . . . . . . . . . . . 155

6 DFT surface simulations of lanthanum hexaboride 159

6.1 Statement on the scientific collaboration . . . . . . . . . . 160

6.2 Experimental measurements on cleaved $\mathrm{LaB}_{6}$ surface samples 160

6.2.1 Experimental methods . . . . . . . . . . . . . 160

6.2 .2 Surface morphology . . . . . . . . . . . . . 161

6.2.3 Scanning tunneling spectroscopy . . . . . . . . . . 165

6.3 Surface simulations . . . . . . . . . . . . . . . . 166

6.3.1 Simulation setup . . . . . . . . . . . . 167

6.3.2 Surface structure after atomic relaxation . . . . . . . 168

6.3.3 Density of states . . . . . . . . . . . . . 169

6.3.4 Electronic states above the surface . . . . . . . . 173

6.4 Discussion . . . . . . . . . . . . . . . 176

$\begin{array}{lll}7 & \text { Summary and perspective } & 179\end{array}$

$\begin{array}{ll}\text { Appendices } & 183\end{array}$

A Supplemental material on the derivation of the Hartree-Fock approximation

A.1 Expectation value of the interaction Hamiltonian with respect to a Slater determinant by Wick's theorem . . . . . . . . . 186

A.2 Expectation values of occupation number operators in the grand canonical ensemble for a non-interacting Hamiltonian . . . . . 189

B Atomic species setups for the CP-PAW simulation code 193

C Supplemental information on the simulations of $\operatorname{PrB}_{6} \quad 195$

D Study of the radial extent of the $5 s, 5 p$, and $4 f$ orbitals of the $\mathrm{La}^{3+}$ ion

E Additional material on the scanning tunneling spectra 203

E.1 The apparent barrier height . . . . . . . . . . . . . 203

E.2 The topography normalization for spatially resolved scanning tunneling spectra . . . . . . . . . . 205

$\begin{array}{ll}\text { Bibliography } & 209\end{array}$ 



\section{Introduction}

A bit more than a hundred years ago the first models have been established, which describe atoms as a composition of positively charged atomic nuclei and negatively charged electrons. With the advent of quantum mechanics in the 1920 s, a quantum mechanical formulation of atomic models followed. However, the subdivision of matter into nuclei and electrons was found to remain sufficient for the description of materials, like solids, liquids, and gases. The interplay of nuclei and electrons on the microscale determines the basic properties of a material. For solid state materials, examples are the crystal structure, mechanical characteristics like rigidity and hardness, chemical inertness, electrical and thermal conductivity, and magnetic behavior.

Although the basic composition of materials by only two general particle species appears to be rather simple, particle-particle interactions can lead to collective physical phenomena in which case material properties can become quite difficult to grasp. One example is the electron-electron interaction. If the electron-electron interaction is weak in comparison with the kinetic energy of the electrons, it is often sufficient to approximate the electron system by a system of non-interacting particles. This is the case in simple metals, where the electrons can move rather freely. In the opposite case of a very strong interaction, electrons tend to localize, as movement would bring electrons closer together and increase the interaction energy. Thus, the system can be described in terms of a Heisenberg model of interacting spins. The most difficult case is represented by a system of electrons, in which kinetic energy and interaction energy are of roughly the same magnitude and where localization and delocalization of electrons meet. This often happens in materials with transition metal or rare-earth constituents where electrons enter $d$ or $f$ orbitals, respectively. Within these orbitals, the electrons are spatially confined and experience rather strong interactions with other electrons in the same shell. This leads to many intriguing physical phenomena, which include Mott-insulating behavior, the Kondo effect, and heavy fermion physics.

A family of materials with a rich variety of physics are the rare-earth hexaborides $R \mathrm{~B}_{6}$. Due to a partially occupied $4 f$ shell, most of the $R \mathrm{~B}_{6}$ belong to the class of strongly correlated electron systems and show features, that are typical for this class of materials. $\mathrm{CeB}_{6}$ is a Kondo lattice with an unusual magnetic phase diagram [1-3]. Other $R \mathrm{~B}_{6}$, including $\mathrm{PrB}_{6}, \mathrm{NdB}_{6}, \mathrm{GdB}_{6}, \mathrm{TbB}_{6}$, $\mathrm{DyB}_{6}$, and $\mathrm{HoB}_{6}$, exhibit antiferromagnetic ordering in the low temperature regime [4]. In contrast, europium hexaboride $\mathrm{EuB}_{6}$ is ferromagnetic and, while most rare-earth hexaborides are metallic, $\mathrm{EuB}_{6}$ exhibits semimetallic behavior $[5,6]$.

In the last decade $\mathrm{SmB}_{6}$ got into the focus of the solid state research com- 
munity when a debate started about $\mathrm{SmB}_{6}$ possibly being a topological Kondo insulator [7]. As topological insulators have an insulating bulk, but a conducting surface due to the presence of non-trivial surface states in the vicinity of the Fermi level, $\mathrm{SmB}_{6}$ was subject to several studies with surface sensitive techniques like scanning tunneling microscopy (STM), scanning tunneling spectroscopy, and also angle-resolved photoemission spectroscopy measurements [8-11]. However, the question about the existence of non-trivial surface states could not be answered satisfactorily. Moreover, even the surface structure remains a matter of debate.

A $R \mathrm{~B}_{6}$ which was associated with topological non-Kondo insulating behavior is $\mathrm{YbB}_{6}[12,13]$. However, results of another study question the presence of non-trivial topological states [14].

The $R \mathrm{~B}_{6}$ also received attention for their diverse physical properties, that make them suitable materials for technological applications. In particular, the $R \mathrm{~B}_{6}$ possess a high electrical conductivity, chemical inertness, hardness, high melting temperatures, and a low volatility at high temperatures [15]. $\mathrm{LaB}_{6}$ and, to a lesser extent, $\mathrm{CeB}_{6}$ are long known for their very low work functions [15-19]. Its excellent thermionic emission properties renders $\mathrm{LaB}_{6}$ the material of choice in high-efficiency thermionic emission applications, e.g. as cathodes in electron microscopes $[15,16,20,21]$. Furthermore, $\mathrm{LaB}_{6}$ was studied as a suitable candidate for arrays of microscale field emitters [22]. Also single-crystalline $\mathrm{LaB}_{6}$ nanowires $[23,24]$ as well as single-crystalline $\mathrm{GdB}_{6}$ nanowires [25] showed promising field-emission characteristics. Further applications include near-infrared absorption from solar light in windows by adding $R \mathrm{~B}_{6}$ nanoparticles to the glass [26, 27]. Also corrosion and wear resistant, as well as decorative $R \mathrm{~B}_{6}$ thin-film coatings have been discussed [28, 29].

Although the $R \mathrm{~B}_{6}$ have been studied over several decades, our knowledge of their physical characteristics remains incomplete. The aforementioned materials $\mathrm{SmB}_{6}$ and $\mathrm{YbB}_{6}$ are only two examples, for which further research is needed. Even for the lighter $R \mathrm{~B}_{6}$ many open questions remain. Photoemission studies [30] of $\mathrm{CeB}_{6}$ showed spectral features that match the expectations for a Kondo system. Notably, a sharp peak was found at the Fermi level, which is attributed to the Kondo resonance peak. Further measurements on $\operatorname{PrB}_{6}$ and $\mathrm{NdB}_{6}$ also showed an enhanced, albeit weaker spectral weight at the Fermi level. However, no signatures of Kondo physics have previously been observed for $\mathrm{PrB}_{6}$ and $\mathrm{NdB}_{6}$, and the origin of the increased spectral weight at the Fermi level remains unclear.

With regard to possible applications in nanotechnology, one can expect that due to the larger surface to bulk ratio in nanostructures, surface features will become more important. However, with the exception of surface investigations of selected $R \mathrm{~B}_{6}$, comprehensive surface studies of the complete rare-earth hexaboride series are lacking. Moreover, recent theoretical predictions of the ground state surface termination of $\mathrm{LaB}_{6}$ [31] disagree with previous measurements on polished and heated samples $[32,33]$. This opens the question whether sample preparations techniques affect the surface structure and how the surface behaves under thermal conditions differing from the regime of thermal 
electron emission.

Their bipartite nature of a valence band of nearly non-interacting electrons on the one hand and strongly interacting electrons within the rare-earth $4 f$ orbitals on the other hand, both of which are coupled via a hybridization, makes materials like the $R \mathrm{~B}_{6}$ quite difficult to handle from a theoretical perspective. A standard technique for material simulations is density functional theory (DFT). In DFT, the ground state energy and ground state electron density of a material are computed in an ab-initio approach. Moreover, the widely applied KohnSham formulation of DFT allows to compute an approximate band structure and an approximate density of states within a one-particle picture. In the Kohn-Sham approach, the exact form of the so-called exchange-correlation energy functional is unknown and needs to be approximated. Early functional models, like the local density approximation (LDA) and its gradient corrections (generalized gradient approximation, GGA) have been successfully applied to various materials. However, they were found unreliable in the case of systems with strongly correlated electrons. For the simulations of such systems, corrections to the simple density functional approximations are computed, usually by treating the strongly localized orbitals in a separate local orbital basis. Well-known examples for such approaches are the so-called LDA+ $U$ and the combination of DFT and dynamical mean field theory, DFT+DMFT. In $\mathrm{LDA}+U$, corrections to the LDA are obtained from a Hartree-Fock treatment of the system of localized orbitals. DFT+DMFT relies on a full many-body approach for the localized orbitals in the framework of DMFT. This has the advantage that the local orbital part is solved in a larger set of basis states in comparison to the DFT treatment. However, a drawback of the DFT+DMFT method is the double-counting problem. While the DMFT part is incorporated to account for interaction effects of the localized orbitals, some exchange and correlation is also treated by the density functional approximation and a double counting correction has to be included. Because the DFT and DMFT are based on fundamentally different formalisms, the exact form of the double counting correction is unknown. Another downside to DFT+DMFT is the large computational cost of the DMFT part, which increases steeply with system size and hence is especially large for systems, in which a full $f$-electron shell needs to be considered.

Another way to improve upon the performance of the LDA/GGA functionals are so-called hybrid functionals, which mix the exchange part of the LDA/GGA functional with a portion of exact exchange. The main improvement of hybrid functionals over LDA/GGA functionals is the correction of the unphysical interaction of a particle with itself, which is an error present in LDA/GGA functionals. Because of the large interaction in localized orbitals, this correction of the self-interaction is especially important for $d$ and $f$ electron systems.

Our research in the context of this thesis is twofold. On the one hand, it is aimed towards a computational approach that allows for reasonably fast and accurate simulations of the $R \mathrm{~B}_{6}$ with occupied $4 f$ electron levels. In recent years the interest in surface characterstics of the $R \mathrm{~B}_{6}$ has been growing. Because of the computational expense of surface simulations, special attention has to be given to the efficiency of the numerical technique. In the context of 
this thesis, we opt for the hybrid functional approach and explore the usability of DFT hybrid functionals in bulk simulations of the light $R \mathrm{~B}_{6}$. In particular, we employ the PBE0r hybrid functional [34].

On the other hand, we investigate the atomic and electronic surface structure of $\mathrm{LaB}_{6}$, which is devoid of occupied $4 f$ orbitals. This part is a joint research project together with experimental collaborators, who conduct scanning tunneling microscopy, scanning tunneling spectroscopy, and low-energy electron diffraction measurements on the cleaved (001) surface of $\mathrm{LaB}_{6}$ singlecrystals. We emphasize that the experimental sample preparation by cleavage at room temperature in an ultra high vacuum distinguishes this surface study from previous studies of the $\mathrm{LaB}_{6}$ surface in which preparation techniques like mechanical surface polishing, sample heating, and argon ion bombardment have been used. $\mathrm{LaB}_{6}$ is the first of the lanthanide hexaborides and does not contain occupied $4 f$ electron levels but captures the electronic structure of the boron constituents. The results of this study can be used as a valuable reference for later surface studies of higher $R \mathrm{~B}_{6}$.

This thesis is organized as follows. In chapter 2 a brief overview of the rareearth hexaborides is given. This includes a description of the basic structure and a summary of the previous research on the rare-earth hexaborides that is most relevant in context of this thesis. Chapter 3 comprises presentations of the theoretical methods that are used in our studies. A focus is put on density functional theory and the theory of the scanning tunneling microscope, which is used to link experimental and theoretical results. The results of DFT bulk simulations on the light rare-earth hexaborides $\mathrm{LaB}_{6}, \mathrm{CeB}_{6}, \mathrm{PrB}_{6}$, and $\mathrm{NdB}_{6}$ are presented in chapter 4 . The simulations have been performed with the PBE0r hybrid functional and the conventional PBE functional, whose results serve as a reference. Especially for the $R \mathrm{~B}_{6}$ with non-zero $4 f$ orbital occupation, the effect of the PBE0r hybrid functional is discussed. An anomalous shape of the density of states is observed. In the subsequent chapter 5, a model system is investigated to explain these findings. The results of simulations on the cleaved $\mathrm{LaB}_{6}(001)$ surface are shown in chapter 6 . These results go hand-in-hand with experimental results and a focus is put on the connection of theoretical and experimental data. The main part of this thesis is concluded with a summary and outlook on future research possibilities in chapter 7 . 


\section{Rare-earth hexaborides}

The rare-earth hexaborides are chemical compounds composed of boron and rare-earth constituents. In this chapter, a brief overview of their physical characteristics is given. Moreover, since one major part of this thesis is geared towards the study of the $\mathrm{LaB}_{6}(001)$ cleavage plane, important findings of prior studies of the $\mathrm{LaB}_{6}$ surface are summarized.

\subsection{Rare earths}

The rare earth elements are a class of materials, comprised of the elements yttrium, scandium, lanthanum, and all of the lanthanides. The discovery of the rare earths is linked to findings of new kinds of minerals in the Swedish Bastnäs mine by A. F. Crondstedt in 1751 and near the Swedish town of Ytterby by C. A. Arrhenius in 1787 [35, 36]. From these and other similar ores, new compound substances were extracted. The term "rare earths" stems from the fact, that the ore was rare and the unknown substances were called "earths" at that time. Over the course of many decades, the members of the group of the rare earth elements could eventually be isolated from these oxides. One exception is promethium, which does not occur naturally due to its radioactive nature and had to be synthesized at a later time. Although the name "rare earths", due to its historic origin, suggests that these elements are scarce, many of them actually occur at a higher concentration in the earth's crust than commonly well known elements such as copper, gold, platinum or lead.

For the lanthanides, the $4 f$ electron count is increased when moving through the lanthanide series. The $4 f$ orbitals are rather localized and have a smaller spatial extension, than the $5 p$ and $6 s$ orbitals. Hence, they are usually not involved in chemical bonding, which explains the similar chemical characteristics of the lanthanides. The lanthanides show a general trend to smaller atomic radii with increasing atomic number, the so-called lanthanide contraction. This can be explained by the larger proton count and the corresponding larger attraction of the outermost electrons towards the core [37].

For solid state material research the lanthanides are highly interesting because in many materials the presence of $4 f$ electrons leads to correlated electron behavior.

\subsection{Boron}

Although boron is the fifth element in the periodic table and thus has a rather simple atomic structure, it took scientists until after 1930 to understand the ba- 
sic physics and chemistry of boron and many of its compounds. The bonding behavior of boron $[37,38]$ is different to that of carbon, its direct neighbor in the periodic table. With its electronic configuration [He] $2 s^{2} 2 p^{1}$, i. e. three valence electrons distributed over four valence orbitals, boron is lacking electrons for chemical bonding according to classical bonding theory and was termed an "electron deficient" material. Multicenter bonds are common in boron compounds, leading to a bonding behavior best described in terms of molecular orbitals. One example is the relatively simple diborane, $\mathrm{B}_{2} \mathrm{H}_{6}$. In this molecule, there are twelve valence electrons - six coming from the boron and six more from the hydrogen atoms. For covalent bonding of the eight atoms to form the diborane molecule, at least seven covalent bonds, and thus a total of 14 electrons are necessary. The lack of electrons is compensated by forming a bridge of two molecular orbitals between the two boron atoms, where one hydrogen atoms contributes to each molecular orbital, resulting in three-center bonds.

Another example is the charged hexaborane molecule $\mathrm{B}_{6} \mathrm{H}_{6}{ }^{2-}$. The molecule is known to have the closo-structure, where the six boron atoms are positioned at the corners of an octahedron with hydrogen atoms connected to the boron corners. Thus, each boron atom has four equidistant boron neighbors and is bonded to one hydrogen atom. Two additional electrons are needed for the stabilization of the octahedral boron arrangement. However, even with the two additional electrons, there are only 26 electrons in the system which is insufficient for covalent bonding between the individual boron constituents. Instead, bonding within the octahedron occurs on the basis of molecular orbitals.
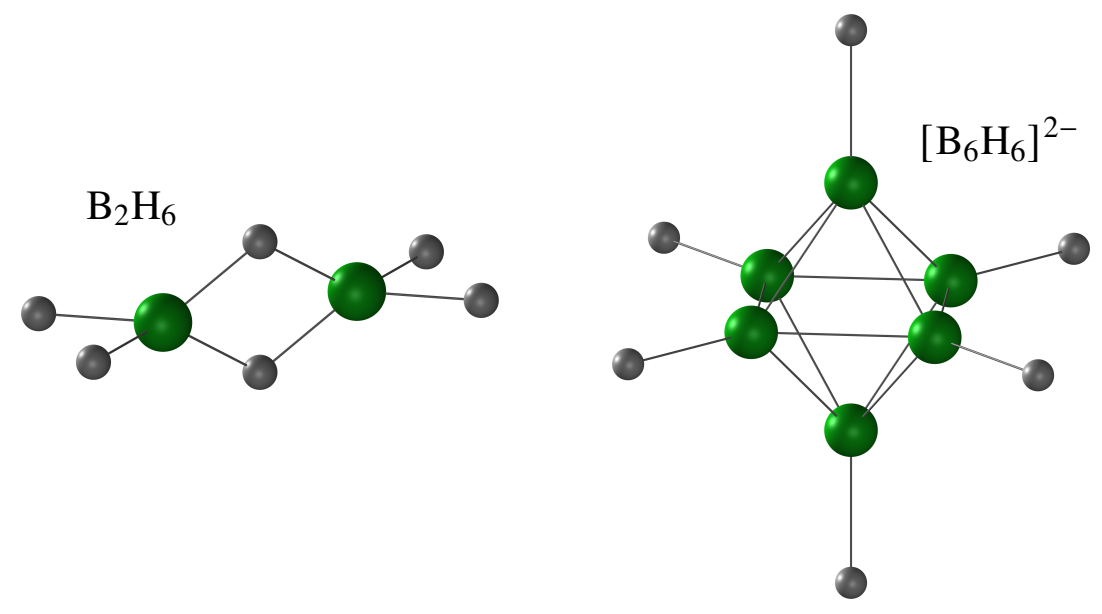

Figure 2.1: Atomic structures of the diborane molecule $\left(\mathrm{B}_{2} \mathrm{H}_{6}\right.$, left $)$ and hexaborane molecule $\left(\left[\mathrm{B}_{6} \mathrm{H}_{6}\right]^{2-}\right.$, right $)$. green: boron. grey: hydrogen.

\subsection{The rare-earth hexaborides}

The materials of interest in this thesis are the rare-earth hexaborides, $R \mathrm{~B}_{6}$, which are a subclass of the borides of type $M \mathrm{~B}_{6}$, where $M$ is a rare earth, an alkali metal, or an alkaline earth metal. All $M \mathrm{~B}_{6}$ have the same simple cubic CsCl-type structure, in which boron octahedra occupy the corners of a cube and 
the metal is located at the cube's center [15, 39]. A scheme of the hexaboride crystal structure is depicted in fig. 2.2. $\mathrm{LaB}_{6}$ has a lattice constant of $4.15 \AA$ [38-42]. When progressing through the $R \mathrm{~B}_{6}$ series towards the heavier rareearths, the lattice constant shows a general trend to shorter values, due to the lanthanide contraction and an adjustment of the boron cage to the smaller size of the rare-earth ion.

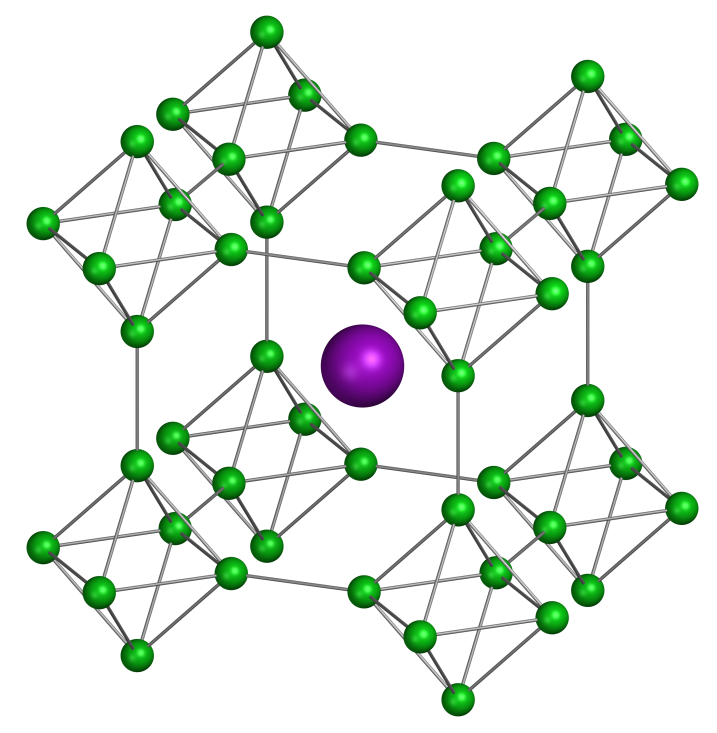

Figure 2.2: Crystal structure of the hexaborides $M \mathrm{~B}_{6}$. Boron constituents in green, the metal ion is colored purple.

Similar to the case of the hexaborane molecule $\mathrm{B}_{6} \mathrm{H}_{6}^{2-}$, the boron octahedra inside a hexaboride take additional electrons that are donated by the metal ion. Note however, that contrary to the $\mathrm{B}_{6} \mathrm{H}_{6}^{2-}$-case an electron transfer of $\sim 1$ electron per metal ion appears to be sufficient to stabilize the boron octahedra inside the hexaboride structure [43]. This explains, why also $\mathrm{KB}_{6}$ with an alkali metal parent is fairly stable. Most rare-earth ions are trivalent within the hexaboride structure. Exceptions are the divalent europium and ytterbium ions in $\mathrm{EuB}_{6}$ and $\mathrm{YbB}_{6}$, respectively. Furthermore, mixed-valency with $\mathrm{Sm}^{2+}$ and $\mathrm{Sm}^{3+}$ ions is found in $\mathrm{SmB}_{6}$.

In the $R \mathrm{~B}_{6}$, the bonding situation according to chemical bonding theory can be described as follows. Outward-pointing molecular orbitals form covalent bonds with neighboring octahedra which results in a rigid boron network structure. The bonding between negatively charged boron octahedra and the positively charged rare-earth ion is of ionic character. For the hexaboride compounds with trivalent rare-earth ions, extra electrons remain for metallic bonding and increased electrical conductivity. Indeed some metal hexaborides are better electrical conductors than their pure parent metals [37, 43]. This hints at a high electron mobility within the boron network.

A feature that makes the hexaborides of the lanthanides appealing from a scientific perspective and, possibly, also for future technological applications is the presence of strongly localized $f$-electrons in the lanthanide ions. The large electron-electron interaction in the $4 f$ shell together with the hybridization coupling between the $4 f$ states and the valence and conduction bands 
makes the rare-earth hexaborides strongly correlated electron systems. A typical signature of correlated electron behavior is the antiferromagnetic ordering at low temperatures of the $R \mathrm{~B}_{6}$ with trivalent rare-earth constituents, which include $\mathrm{PrB}_{6}, \mathrm{NdB}_{6}, \mathrm{GdB}_{6}, \mathrm{TbB}_{6}, \mathrm{DyB}_{6}$, and $\mathrm{HoB}_{6}$ [4]. In contrast, europium ions within $\mathrm{EuB}_{6}$ are divalent and $\mathrm{EuB}_{6}$ behaves as a semimetal. Moreover, two ferromagnetic phases with orientations along two different axes have been observed, with transition temperatures of $\sim 15 \mathrm{~K}$ and $\sim 12.5 \mathrm{~K}$, respectively [5, 6]. $\mathrm{CeB}_{6}$ is a Kondo lattice and shows different magnetic phases at cryogenic temperatures. Above $3.2 \mathrm{~K}$ the resistivity curve shows a $\log T$-dependence, typical for Kondo systems. At temperatures below $3.2 \mathrm{~K}$, a sharp decrease in the resistivity is found and a magnetic phase of antiferroquadrupolar ordering has been identified at temperatures temperatures $2.4 \mathrm{~K}<T<3.2 \mathrm{~K}$. Measurements on $\mathrm{CeB}_{6}$ inside a magnetic field, in which the resistivity maximum is shifted, indicate a competition between the antiferroquadrupolar phase and the Kondo state [2]. Below $2.4 \mathrm{~K}$ a modulated magnetic ordering phase was found. In the mixed-valent $\mathrm{SmB}_{6}$, samarium ions are either $\mathrm{Sm}^{2+}$ or $\mathrm{Sm}^{3+} . \mathrm{YbB}_{6}$ contains divalent ytterbium ions. Both, $\mathrm{SmB}_{6}$ and $\mathrm{YbB}_{6}$ have been associated with topological behavior. However, whether $\mathrm{SmB}_{6}$ is a topological Kondo insulator [7-11] and whether $\mathrm{YbB}_{6}$ is a non-Kondo topological insulator [12-14] is matter of debate.

\subsection{The $\mathrm{LaB}_{6}$ surface}

$\mathrm{LaB}_{6}$ is long known for its excellent thermionic electron emission properties. Some years after pioneering studies of Lafferty on boride cathodes [15], in the 1960s new types of $\mathrm{LaB}_{6}$ electron emitters arrived as an alternative for tungsten hairpin cathodes for applications in electron microscopes [16, 20, 21]. $\mathrm{LaB}_{6}$ has several characteristics that render it highly applicable as an electron beam source. First, the (001) surface of $\mathrm{LaB}_{6}$ possesses a low work function of $\sim 2.6 \mathrm{eV}$ [15-19], which allows for high-brightness electron beams. Second, $\mathrm{LaB}_{6}$ shows a high thermal stability. At operation temperatures of $\sim 1600{ }^{\circ} \mathrm{C}$, where very high electron beam current densities are available, $\mathrm{LaB}_{6}$ has a rather low evaporation rate under vacuum conditions [16]. Furthermore, $\mathrm{LaB}_{6}$ proved to be resistant against chemical poisoning [44]. Both, the high thermal and chemical stability, allow for long cathode life times.

Because of its applicability as a thermionic electron emitter, $\mathrm{LaB}_{6}$ was subject to several studies, many of which have been performed from the mid 1970s to the mid 1980s. A crucial point of these studies was the determination of the surface's atomic structure, such as surface composition and surface termination. Besides the (001) surface, other crystal surfaces, like the (011) and (111) surfaces, have been investigated. However, in this thesis we restrict the discussion to results for the La (001) surface and, in the following, give a brief overview of selected findings. It is important to note that in these studies the preparation of the $\mathrm{LaB}_{6}$ crystal samples usually involved surface polishing and heat treatment for thermal cleaning of the surface.

Auger electron spectroscopy (AES) measurements on the La (001) surface 
were indicative of a lanthanum surface termination, as the determined atomic $\mathrm{B} / \mathrm{La}$ ratios at the surface were smaller than the bulk stoichiometric value $[17,45-48]$. In a study of the $\mathrm{LaB}_{6}(001)$ surface with angle-resolved $\mathrm{X}$ ray photoemission spectroscopy (AR-XPS), the XPS intensity peak ratio of lanthanum $4 d$ and boron $1 s$ states was analyzed [49]. Values of the ratio $I(\mathrm{La} 4 d) / I(\mathrm{~B} 1 s)$ were reported to be between 1 and 2 for angles of $0^{\circ}$ to $\sim 75^{\circ}$ from the surface normal and rising sharply for larger angles. With larger angles being more sensitive to the surface and showing a larger portion of lanthanum states, it was concluded that the surface is lanthanum terminated, which supports the AES findings.

In several studies low-energy electron diffraction (LEED) measurements of the $\mathrm{LaB}_{6}$ surface were performed. For clean $\mathrm{LaB}_{6}(001)$ surfaces a square pattern was observed [18, 45, 46, 49]. Together with the AES findings this gives evidence for an unreconstructed $(1 \times 1)$ lanthanum terminated surface. For a surface termination of this kind, the covalent bonds of the boron network that are perpendicular to the surface plane are broken at the surface level, leaving unpaired boron $s p$ hybrid dangling bonds. In their study with angular-resolved ultraviolet photoelectron spectroscopy, Aono et al. found a surface feature at $\sim 2 \mathrm{eV}$ below the Fermi level, which they attributed to the boron dangling bonds [50]. Furthermore, based on their data they suggested that the boron dangling bonds bind into the states of a lanthanum ion lying above the boron octahedra at the surface level.

With the advent of scanning tunneling microscopy (STM), surface investigations with atomic resolution became feasible and first STM studies on the surface of lanthanum hexaboride have been conducted by Ozcomert and Trenary [32, 33]. With an STM they scanned the (001) surface of a $\mathrm{LaB}_{6}$ crystal that was polished and heated to temperatures of $2200 \mathrm{~K}$ several times prior to the transfer to the STM. On a large scale the STM images showed terraces separated by steps, each of roughly $4 \AA$ height, matching the lattice constant of $4.15 \AA$. As each step was found to be of one-lattice-constant height, all terraces have to have the same termination, which was claimed to be a La termination, based on the results of the previous studies. Moreover, close-up images with atomic resolution were achieved, showing a $(1 \times 1)$ structure with a spacing of $\sim 4 \AA$.

A theoretical study of the lanthanum terminated non-reconstructed $\mathrm{LaB}_{6}$ (001) surface with density functional theory was conducted by Monnier and Delley [51]. They found a surface band with contributions from the topmost boron constituents and the lanthanum surface ions in agreement with the photoemission study of Aono et al. Furthermore, in their simulation the lanthanum surface layer relaxed inwards by $6.5 \%$.

In another theoretical work Uijttewaal et al. studied unreconstructed (001), (011), (111), (112), and (012) surfaces of $\mathrm{LaB}_{6}$ with DFT, taking into account different surface terminations [52]. With a focus on work functions and surface stability, they concluded that the most stable surfaces are either the boron terminated (111) surface or the lanthanum terminated (001) surface, depending on the material's chemical potential. The (001) surface was found to have the lowest work function. 
The aforementioned experimental studies found a non-reconstructed lanthanum terminated (001) surface and are in good agreement with theoretical work. Sample preparation and measurements in the experiments were performed under conditions that are typical for the thermionic emission use case. However, preparations techniques like surface polishing, thermal treatment, and argon ion bombardment affect the surface and might be responsible for the observed surface structure. With applications apart from thermionic emission the nature of the $\mathrm{LaB}_{6}$ surface at room temperature becomes relevant.

Recently, a DFT-based study of the $\mathrm{LaB}_{6}$ surface found a rowlike arrangement of the lanthanum ions at the surface to be energetically favorable [31]. In the study the surface energies of surface slabs with four different surface terminations have been computed. The selected surface terminations are a $(1 \times 1)$ polar termination, $a(1 \times 2)$ chainlike termination, $a(\sqrt{2} \times \sqrt{2})$ diagonal gridlike termination, and a $(1 \times 4)$ termination, where rows of two adjacent lanthanum ions are separated by rows of two empty lanthanum spots. It was found that the surface energies were lowest for the $(1 \times 4)$ and the $(1 \times 2)$ terminations, with values of $14.4048(9) \mathrm{eV} / \mathrm{nm}^{2}$ and $14.5274(78) \mathrm{eV} / \mathrm{nm}^{2}$, respectively, while the surface energy of the $(1 \times 1)$ surface is stated to be $15.3477(26) \mathrm{eV} / \mathrm{nm}^{2}$, and the value for the $(\sqrt{2} \times \sqrt{2})$ termination is $15.5542(53) \mathrm{eV} / \mathrm{nm}^{2}$. With these values in mind one should expect chains of lanthanum ions at the $\mathrm{LaB}_{6}(001)$ surface instead of the commonly reported $(1 \times 1)$ structure. 


\section{$3 \mid$ Methods}

In this chapter the theoretical foundations for this thesis are presented. This includes a brief description of many-particle physics in a solid state material, which at the same time is used to introduce some basic notation. Subsequently, the Hartree-Fock method is summarized for later reference. This is followed by a description of the theoretical techniques used for the material simulations in the context of this thesis: density functional theory (DFT), Car-Parrinello dynamics, and the projector augmented-wave method (PAW). In the last section, theoretical aspects of the scanning tunneling microscope are discussed, which will be used to connect experimental results from scanning tunneling microscopy and scanning tunneling spectroscopy to the results of DFT surface simulations.

\subsection{Electronic correlations}

Two events are said to be correlated, if there is some link between them, such that one event is influenced by the other, a third event influences both of them or the two events just randomly have something in common. Not only events, but, as examples, also two states, properties, or quantities can be correlated.

One of the main fields in physics, where correlations are typically of major importance, are studies of many-particle systems. As an example, consider a system of classical particles of the same kind, where each particle can move freely and is not influenced by the presence of any other particle. Calculating a particle's trajectory is very easy, if location and momentum of the particle are known at some time. The particle's future behavior only depends on the particle's own current state. However, if collisions between particles are possible, a particle's future position and momentum also depend on the positions and momenta of the other particles, since it might be deflected from its "free" path by colliding with other particles. Thus, the particles don't move independently anymore, they are correlated. Mathematically this means that in the differential equation which describes a particle's motion, there is a term that couples one or more properties of the particle with one or more properties of the other, colliding particle. One speaks of a two-body term. Because of these two-body terms, the differential equation becomes much harder or even impossible to solve, the reason often being technical restrictions.

Analogous to the classical case described above, there are two-body terms also in the quantum regime. Most prominent for solid state materials are the electron-electron Coulomb repulsion and electron-phonon coupling (in this thesis we will not be concerned with the latter). The effect of the Coulomb interaction as a two-body term onto the electron density, which is an important 
reoccurring quantity throughout this thesis, is especially illustrative. Its influence can be clarified with the use of the one-electron density $n^{(1)}(\vec{r})$ and the two-electron density $n^{(2)}\left(\vec{r}, \vec{r}^{\prime}\right)$. The former can be regarded as the probability of an electron being at location $\vec{r}$ and the latter is a measure of the probability of having one electron at coordinates $\vec{r}$ and another electron at location $\vec{r}^{\prime}$. If the electrons were completely independent particles and were not even subject to the Pauli principle, clearly $n^{(2)}\left(\vec{r}, \vec{r}^{\prime}\right)=n^{(1)}(\vec{r}) n^{(1)}\left(\vec{r}^{\prime}\right)$, which is analogous to the case of two independent random variables. However, electrons are generally not independent particles due to exchange and their Coulomb repulsion. It is important to distinguish between exchange and electronic correlations, as exchange originates from the formulation of fermion physics in terms of indistinguishable particles and does not have a classical analog. On the other hand, electronic correlations originate from the true electron-electron repulsion due to the true Coulomb interaction. Hence, even if the electrons are assumed to be non-interacting, they are not independent particles due to the exchange.

Analogous to probability theory, the two-electron density can be decomposed into the one-electron density and the conditional density $n\left(\vec{r}^{\prime} \mid \vec{r}\right)$ to have an electron at location $\vec{r}^{\prime}$ when there is an electron at location $\vec{r}$ :

$$
n^{(2)}\left(\vec{r}, \vec{r}^{\prime}\right)=n^{(1)}(\vec{r}) n\left(\vec{r}^{\prime} \mid \vec{r}\right)
$$

Since the Coulomb interaction is repulsive, the presence of an electron at $\vec{r}$ reduces the probability of having another electron close by. This is commonly expressed through

$$
n\left(\vec{r}^{\prime} \mid \vec{r}\right)=n^{(1)}\left(\vec{r}^{\prime}\right)+h\left(\vec{r}, \vec{r}^{\prime}\right),
$$

where the hole-function $h\left(\vec{r}, \vec{r}^{\prime}\right)$ represents the so-called exchange-correlation hole due to the two-particle interaction and describes the deviation from the case of independent particles (in this context even without being subject to the Pauli principle). Hence, the two-particle density becomes

$$
n^{(2)}\left(\vec{r}, \vec{r}^{\prime}\right)=n^{(1)}(\vec{r})\left(n^{(1)}\left(\vec{r}^{\prime}\right)+h\left(\vec{r}, \vec{r}^{\prime}\right)\right)
$$

In the course of this thesis, eq. (3.3) will be used for splitting the electronelectron interaction into an independent-particle contribution and an exchangecorrelation part, which "corrects" the independent-particle term.

In the remainder of this section, for later reference, we state sum rules for the one-particle density, the two-particle density, and the hole function. The integral of the one-particle density over all space gives the electron number. For an $N$-particle system we have

$$
\int \mathrm{d} \vec{r} n^{(1)}(\vec{r})=N
$$

Moreover, the conditional density $n\left(\vec{r}^{\prime} \mid \vec{r}\right)$ has the property

$$
\int \mathrm{d} \vec{r}^{\prime} n\left(\vec{r}^{\prime} \mid \vec{r}\right)=N-1
$$


This is because if one electron is at location $\vec{r}$ we have a total of $N-1$ other electrons distributed in space. Formulated in other terms one can also say that an electron which is found at location $\vec{r}$ misses from the rest of the system. This is an explanation for the sum rule for the hole function,

$$
\int \mathrm{d} \vec{r}^{\prime} h\left(\vec{r}, \vec{r}^{\prime}\right)=-1
$$

which can be found by combining eqs. (3.2), (3.4) and (3.5). For completeness we also state that for the two-particle density we find

$$
\int \mathrm{d} \vec{r} \int \mathrm{d} \vec{r}^{\prime} n^{(2)}\left(\vec{r}, \vec{r}^{\prime}\right)=N(N-1) .
$$

\subsection{Many-particle physics}

The theoretical description of materials like solid state materials, liquids and gases usually is based on two basic types of particles: atomic nuclei and electrons. Any substance can be composed of selected nuclei with the correct nuclear charge $Z e$ for the desired material, and the right number of electrons. From a quantum mechanical point of view, this situation can be expressed by the fundamental Hamiltonian

$$
\begin{aligned}
\hat{H}= & \hat{T}_{\text {nuc }}+\hat{W}_{\text {nuc-nuc }}+\hat{T}_{\mathrm{el}}+\hat{W}_{\mathrm{el}-\mathrm{el}}+\hat{W}_{\mathrm{el}-\mathrm{el}}+\hat{W}_{\text {nuc-el }} \\
= & \underbrace{\sum_{I=1}^{N_{\text {nuc }}} \frac{\hat{\vec{P}}^{2}}{2 M_{I}}}_{\hat{T}_{\mathrm{nuc}}}+\underbrace{\frac{e^{2}}{4 \pi \varepsilon_{0}} \sum_{I=1}^{N_{\text {nuc }}} \sum_{J<I}^{N_{\text {nuc }}} \frac{Z_{I} Z_{J}}{\left|\hat{\vec{R}}_{I}-\hat{\vec{R}}_{J}\right|}}_{\hat{W}_{\text {nuc-nuc }}} \\
& +\underbrace{\sum_{i=1}^{N_{\mathrm{el}}} \frac{\hat{\vec{p}}_{i}^{2}}{2 m_{i}}}_{\hat{T}_{\mathrm{el}}}+\underbrace{\frac{e^{2}}{4 \pi \varepsilon_{0}} \sum_{i=1}^{N_{\mathrm{el}}} \sum_{j<i}^{N_{\mathrm{el}}} \frac{1}{\left|\hat{\vec{r}}_{i}-\hat{\vec{r}}_{j}\right|}}_{\hat{W}_{\mathrm{el}-\mathrm{el}}} \underbrace{-\frac{e^{2}}{4 \pi \varepsilon_{0}} \sum_{I=1}^{N_{\text {nuc }}} \sum_{j=1}^{N_{\mathrm{el}}} \frac{Z_{I}}{\left|\hat{\vec{R}}_{I}-\hat{\vec{r}}_{j}\right|}}_{\hat{W}_{\text {nuc-el }}} .
\end{aligned}
$$

$\hat{T}_{\text {nuc }}$ is the kinetic energy operator of the nuclei, where $\hat{\vec{P}}_{I}$ and $M_{I}$ denote the momentum operator and the mass of the $I$-th nucleus. Similarly, $\hat{T}_{\mathrm{el}}$ is the kinetic energy operator of the electrons, where $m_{e}$ is the electron mass and $\hat{\vec{p}}_{i}$ denotes the momentum operator of the $i$-th electron. The only fundamental force of significant magnitude is the electromagnetic force. This is expressed by the three interaction operators $\hat{W}_{\text {nuc-nuc }}, \hat{W}_{\text {el-el }}$, and $\hat{W}_{\text {nuc-el }}$, representing the Coulomb interaction between the nuclei, between the electrons, and between nuclei and electrons, respectively. $\hat{\vec{R}}_{I}$ and $\hat{\vec{r}}_{j}$ denote the position operators of the $I$-th nucleus and the $j$-th electron. $Z_{I}$ is the atomic number of the $I$-th nucleus.

Generally, the physical systems to be investigated contain so many particles that the Schrödinger equation for the raw Hamiltonian (3.8) cannot be solved. 
To proceed, often simplifications are introduced. One simplification is the treatment of the nuclei and their most tightly bound electrons as single entities. This is justified when tightly bound "core" electrons have a negligible impact on the overall physical properties of the material which is often the case in solid state materials. In this case, the nuclei translate to ions and the number of electronic degrees of freedom in the system is reduced.

\subsection{The Born-Oppenheimer approximation}

A major simplification for the many-particle problem of solid state theory is the Born-Oppenheimer approximation, which was introduced by Born and Oppenheimer [53] and refined later by Born and Huang [54]. In the BornOppenheimer approximation the electronic and nuclear degrees of freedom are separated.

For a separation of the electronic and nuclear degrees of freedom, it is sensible to define the Hamiltonian

$$
\begin{aligned}
\hat{H}_{\mathrm{BO}}(\{\vec{R}\})= & \sum_{i=1}^{N_{\mathrm{el}}} \frac{\hat{\vec{p}}_{i}^{2}}{2 m_{i}}+\frac{e^{2}}{4 \pi \varepsilon_{0}} \sum_{i=1}^{N_{\mathrm{el}}} \sum_{j<i}^{N_{\mathrm{el}}} \frac{1}{\left|\hat{\vec{r}}_{i}-\hat{\vec{r}}_{j}\right|} \\
& -\frac{e^{2}}{4 \pi \varepsilon_{0}} \sum_{I=1}^{N_{\mathrm{nuc}}} \sum_{j=1}^{N_{\mathrm{el}}} \frac{Z_{I}}{\left|\vec{R}_{I}-\hat{\vec{r}}_{j}\right|}+\frac{e^{2}}{4 \pi \varepsilon_{0}} \sum_{I=1}^{N_{\mathrm{nuc}}} \sum_{J<I}^{N_{\mathrm{nuc}}} \frac{Z_{I} Z_{J}}{\left|\vec{R}_{I}-\vec{R}_{J}\right|},
\end{aligned}
$$

which is stripped of nuclear momenta and which is a Hamiltonian for the electronic system for a fixed set of nuclear positions $\{\vec{R}\}$. Since the positions of the nuclei are fixed, the Coulomb interaction between the nuclei is reduced to a constant for the electronic problem. With the Hamiltonian (3.9) we can set up a Schrödinger equation for the electronic system,

$$
\hat{H}_{\mathrm{BO}}(\{\vec{R}\})\left|\psi_{n}^{\mathrm{BO}}(\{\vec{R}\})\right\rangle=\varepsilon_{n}^{\mathrm{BO}}(\{\vec{R}\})\left|\psi_{n}^{\mathrm{BO}}(\{\vec{R}\})\right\rangle,
$$

where $\left|\psi_{n}^{\mathrm{BO}}(\{\vec{R}\})\right\rangle$ denotes a state of the electronic system with energy $\varepsilon_{n}^{\mathrm{BO}}(\{\vec{R}\})$ for fixed nuclear positions $\{\vec{R}\}$.

Further, for an arbitrary set of nuclear coordinates $\{\vec{R}\}$ and a time $t$, a wave function of the solid state system can be expanded with respect to the eigenstates $\left|\psi_{n}^{\mathrm{BO}}(\{\vec{R}\})\right\rangle$ of the Hamiltonian (3.9) [54-56]. In the coordinate space representation we find

$$
\Psi(\{\vec{r}\},\{\vec{R}\}, t)=\sum_{n} \psi_{n}^{\mathrm{BO}}(\{\vec{r}\} ;\{\vec{R}\}) \phi_{n}(\{\vec{R}\}, t),
$$

where the functions $\phi_{n}(\{\vec{R}\}, t)$ are the expansion coefficients and $\{\vec{r}\}$ is the set of electron positions. The electronic wave functions are a complete set of functions and are chosen to be orthonormal,

$$
\iiint \mathrm{d} \vec{r}_{1} \ldots \mathrm{d} \vec{r}_{N_{\mathrm{el}}} \psi_{m}^{\mathrm{BO} *}(\{\vec{r}\} ;\{\vec{R}\}) \psi_{n}^{\mathrm{BO}}(\{\vec{r}\} ;\{\vec{R}\})=\delta_{m n} .
$$


Next, we insert the expansion (3.11) into the time-dependent Schrödinger equation of the solid state system. We let the Hamiltonian (3.8) act on eq. (3.11) and subsequently multiply with $\psi_{m}^{\mathrm{BO} *}(\{\vec{r}\} ;\{\vec{R}\})$. An integration over the electronic coordinates and exploiting eqs. (3.9) and (3.12) leads to the following equation for the nuclear functions,

$$
\begin{aligned}
i \hbar \frac{\partial}{\partial t} \phi_{m}(\{\vec{R}\}, t)=[ & \left.\hat{T}_{\text {nuc }}+\varepsilon_{m}^{\mathrm{BO}}(\{\vec{R}\})\right] \phi_{m}(\{\vec{R}\}, t) \\
& -\sum_{n}\left(\hat{A}_{m n}+\hat{B}_{m n}\right) \phi_{n}(\{\vec{R}\}, t) .
\end{aligned}
$$

Note that the chain rule of differentiation produces the last term with the operators

$$
\begin{aligned}
& \hat{A}_{m n}(\{\vec{R}\})=\sum_{I} \frac{\hbar^{2}}{M_{I}}\left\langle\psi_{m}^{\mathrm{BO}}(\{\vec{R}\})\left|\nabla_{\vec{R}_{I}}\right| \psi_{n}^{\mathrm{BO}}(\{\vec{R}\})\right\rangle \nabla_{\vec{R}_{I}}, \\
& \hat{B}_{m n}(\{\vec{R}\})=\sum_{I} \frac{\hbar^{2}}{2 M_{I}}\left\langle\psi_{m}^{\mathrm{BO}}(\{\vec{R}\})\left|\nabla_{\vec{R}_{I}}^{2}\right| \psi_{n}^{\mathrm{BO}}(\{\vec{R}\})\right\rangle \hat{1},
\end{aligned}
$$

where we exploited the coordinate space-representation of the nuclear momentum operator $\hat{\vec{P}}_{I}=-i \hbar \nabla_{\vec{R}_{I}}$. The operators $\hat{A}_{m n}$ and $\hat{B}_{m n}$ couple the electronic and nuclear wave functions that are associated with different $\varepsilon_{m}^{\mathrm{BO}}$.

So far we did not make use of any approximation. However, at this point we introduce the Born-Oppenheimer approximation, which is to neglect the last term of eq. (3.13). This removes the explicit dependence of equation eq. (3.13) on the electronic states $\left|\psi_{n}(\{\vec{R}\})\right\rangle$ and the nuclear states $\phi_{n}(\{\vec{R}\}, t)$ that do not belong to the eigenvalue $\varepsilon_{m}^{\mathrm{BO}}$ and gives a Schrödinger equation for the nuclear wave function

$$
i \hbar \frac{\partial}{\partial t} \phi_{m}(\{\vec{R}\}, t)=\left[\hat{T}_{\mathrm{nuc}}+\varepsilon_{m}^{\mathrm{BO}}(\{\vec{R}\})\right] \phi_{m}(\{\vec{R}\}, t) .
$$

In this Schrödinger equation the nuclear motion takes place on a potential surface given by the eigenvalue $\varepsilon_{m}^{\mathrm{BO}}(\{\vec{R}\})$ of the Hamiltonian (3.9). $\varepsilon_{m}^{\mathrm{BO}}(\{\vec{R}\})$ is also called a Born-Oppenheimer surface.

When the operators $\hat{A}_{m n}$ and $\hat{B}_{m n}$ are neglected, a wave function of the solid state system can be written in terms of a product wave function

$$
\Psi_{n}(\{\vec{r}\},\{\vec{R}\}, t)=\psi_{n}^{\mathrm{BO}}(\{\vec{r}\} ;\{\vec{R}\}) \phi_{n}(\{\vec{R}\}, t),
$$

where $\phi_{n}(\{\vec{R}\}, t)$ describes the motion of the nuclei and $\psi_{n}^{\mathrm{BO}}(\{\vec{r}\} ;\{\vec{R}\})$ is the electronic state of the system for the nuclei being fixed at their current positions. Upon nuclear motion the electron wave functions adapt instantly to the new set of nuclear coordinates. The electron motion is said to be adiabatic. Note that the neglect of the couplings $\hat{A}_{m n}$ and $\hat{B}_{m n}$ has the following consequence. If the system started on the Born-Oppenheimer surface $\varepsilon_{n}^{\mathrm{BO}}$, it will not leave this surface at any point of the time evolution given by eqs. (3.10) and (3.13).

Born and Oppenheimer [53, 54] used the fact that the nuclear mass is 
much larger than the electron mass and treated the Schrödinger equation with the Hamiltonian (3.8) in a perturbative approach with the small parameter $\kappa=\sqrt[4]{m_{e} / M_{0}}$, where $M_{0}$ is a mean nuclear mass. They showed that the wave function takes the form (3.17), if the perturbative expansion of the wave function is broken off after the second order in $\kappa$. Thus, the Born-Oppenheimer approximation is justified for $m_{e} \ll M_{0}$, in which case the electron dynamics is much faster than the motion of the nuclei. It is, however, important to mention that the Born-Oppenheimer approximation breaks down if different Born-Oppenheimer surfaces come close or cross.

The Born-Oppenheimer approximation is often used in conjunction with a classical approximation for the dynamics of the nuclei. In this case the dynamics of a nucleus is governed by the classical equation of motion (see e.g. $[56,57])$

$$
M_{I} \frac{\partial^{2} \vec{R}_{I}}{\partial t^{2}}=-\nabla_{\vec{R}_{I}} \varepsilon_{m}^{\mathrm{BO}}(\{\vec{R}\}) .
$$

The combination of an electronic structure method to determine the BornOppenheimer surface $\varepsilon_{m}^{\mathrm{BO}}(\{\vec{R}\})$ and the classical treatment of the nuclear motion is known as an ab-initio molecular dynamics approach. As we will see, such an approach is used in the CP-PAW simulation code that is employed in the context of this thesis.

\subsection{The electronic Hamiltonian}

In this section we establish some of the basic notation used throughout this thesis. Analogous to eq. (3.9) we define the electronic Hamiltonian as

$$
\hat{H}=\underbrace{-\sum_{i=1}^{N} \frac{\hat{\vec{p}}_{i}^{2}}{2 m}}_{\hat{T}}+\underbrace{\sum_{i=1}^{N} v_{\mathrm{ext}}\left(\hat{\vec{r}}_{i}\right)}_{\hat{V}}+\underbrace{\frac{1}{2} \sum_{i=1}^{N} \sum_{j \neq i} w\left(\hat{\vec{r}}_{i}, \hat{\vec{r}}_{j}\right)}_{\hat{W}},
$$

which also defines the operators for the kinetic energy of the electrons $\hat{T}$, the potential energy $\hat{V}$, and the interaction energy $\hat{W}$, respectively. The electronelectron Coulomb interaction is

$$
w\left(\hat{\vec{r}}_{i}, \hat{\vec{r}}_{j}\right)=\frac{e^{2}}{4 \pi \varepsilon_{0}} \frac{1}{\left|\hat{\vec{r}}_{i}-\hat{\vec{r}}_{j}\right|}
$$

and the external potential due to the electron-ion Coulomb interaction reads

$$
v_{\mathrm{ext}}(\hat{\vec{r}})=-\frac{e^{2}}{4 \pi \varepsilon_{0}} \sum_{J=1}^{N_{\mathrm{ion}}} Z_{J} w\left(\hat{\vec{r}}-\vec{R}_{J}\right),
$$

where $Z_{J}$ is the ion charge number. The ion-ion interaction in eq. (3.9) is a constant energy contribution for fixed ion positions. Hence, it only introduces a constant energy shift on the energy due to the other contributions and is 
neglected in the Hamiltonian (3.19). The Hamiltonian (3.19) consequently is composed of electronic contributions only and describes a system of electrons at fixed ion positions. Finding the ground state energy as well as the eigenstates of the electronic Hamiltonian (3.19) is often the core element in theoretical solid state research.

Often, the Hamiltonian (3.19) is represented in the formalism of second quantization, where it takes the form

$$
\hat{H}=\sum_{a b} h_{a b} \hat{c}_{a}^{\dagger} \hat{c}_{b}+\frac{1}{2} \sum_{a b c d} U_{a b d c} \hat{c}_{a}^{\dagger} \hat{c}_{b}^{\dagger} \hat{c}_{c} \hat{c}_{d}
$$

$\hat{c}_{a}$ and $\hat{c}_{a}^{\dagger}$ are creation and annihilation operators for a particle in state $a$. The matrix $h_{a b}$ in the single-particle term contains all information about the kinetic energy and the external potential and is given via

$$
h_{a b}=\sum_{\sigma} \int \mathrm{d} \vec{r} \phi_{a}^{*}(\vec{r}, \sigma)\left[-\frac{\hbar^{2}}{2 m} \nabla_{\vec{r}}^{2}+v_{\mathrm{ext}}(\vec{r})\right] \phi_{b}(\vec{r}, \sigma),
$$

where $\phi_{a}(\vec{r}, \sigma)$ denotes the one-particle wave function of a state with quantum number $a$ at local coordinate $\vec{r}$ and spin coordinate $\sigma$. The two-particle interaction $\hat{W}$ is represented by the second term in eq. (3.22), where the tensor elements $U_{a b c d}$ are given by

$$
U_{a b c d}=\sum_{\sigma_{1} \sigma_{2}} \iint \mathrm{d} \vec{r}_{1} \mathrm{~d} \vec{r}_{2} \phi_{a}^{*}\left(\vec{r}_{1}, \sigma_{1}\right) \phi_{b}^{*}\left(\vec{r}_{2}, \sigma_{2}\right) w\left(\vec{r}_{1}, \vec{r}_{2}\right) \phi_{c}\left(\vec{r}_{1}, \sigma_{1}\right) \phi_{d}\left(\vec{r}_{2}, \sigma_{2}\right) .
$$

Note that the interaction tensor with the interaction (3.20), for which $w\left(\vec{r}_{2}, \vec{r}_{1}\right)=$ $w\left(\vec{r}_{1}, \vec{r}_{2}\right)$, has the symmetry

$$
U_{a b c d}=U_{b a d c}
$$

which can be verified by relabeling the coordinates of the wave functions. Further, if the quantum numbers of the wave functions are identified with spin orbitals, $a \rightarrow m \sigma, b \rightarrow m^{\prime} \sigma^{\prime}, c \rightarrow m^{\prime \prime} \sigma^{\prime \prime}, d \rightarrow m^{\prime \prime \prime} \sigma^{\prime \prime \prime}$, where $m$ is an orbital quantum number, the elements of the interaction tensor become

$$
\begin{aligned}
U_{m \sigma, m^{\prime} \sigma^{\prime}, m^{\prime \prime} \sigma^{\prime \prime}, m^{\prime \prime \prime} \sigma^{\prime \prime \prime}=} & \sum_{\sigma_{1} \sigma_{2}} \iint \mathrm{d} \vec{r}_{1} \mathrm{~d} \vec{r}_{2} \phi_{m \sigma}^{*}\left(\vec{r}_{1}, \sigma_{1}\right) \phi_{m^{\prime} \sigma^{\prime}}^{*}\left(\vec{r}_{2}, \sigma_{2}\right) \\
& \times w\left(\vec{r}_{1}, \vec{r}_{2}\right) \phi_{m^{\prime \prime} \sigma^{\prime \prime}}\left(\vec{r}_{1}, \sigma_{1}\right) \phi_{m^{\prime \prime \prime} \sigma^{\prime \prime \prime}}\left(\vec{r}_{2}, \sigma_{2}\right) \\
= & \sum_{\sigma_{1} \sigma_{2}} \iint \mathrm{d} \vec{r}_{1} \mathrm{~d} \vec{r}_{2} \phi_{m \sigma}^{*}\left(\vec{r}_{1}, \sigma\right) \phi_{m^{\prime} \sigma^{\prime}}^{*}\left(\vec{r}_{2}, \sigma^{\prime}\right) \\
& \times w\left(\vec{r}_{1}, \vec{r}_{2}\right) \phi_{m^{\prime \prime} \sigma^{\prime \prime}}\left(\vec{r}_{1}, \sigma^{\prime \prime}\right) \phi_{m^{\prime \prime \prime} \sigma^{\prime \prime \prime}}\left(\vec{r}_{2}, \sigma^{\prime \prime \prime}\right) \\
& \times \delta_{\sigma \sigma_{1}} \delta_{\sigma^{\prime} \sigma_{2}} \delta_{\sigma^{\prime \prime} \sigma_{1}} \delta_{\sigma^{\prime \prime \prime} \sigma_{2}}
\end{aligned}
$$




$$
\begin{aligned}
& =\iint \mathrm{d} \vec{r}_{1} \mathrm{~d} \vec{r}_{2} \phi_{m \sigma}^{*}\left(\vec{r}_{1}, \sigma\right) \phi_{m^{\prime} \sigma^{\prime}}^{*}\left(\vec{r}_{2}, \sigma^{\prime}\right) \\
& \quad \times w\left(\vec{r}_{1}, \vec{r}_{2}\right) \phi_{m^{\prime \prime} \sigma}\left(\vec{r}_{1}, \sigma\right) \phi_{m^{\prime \prime \prime} \sigma^{\prime}}\left(\vec{r}_{2}, \sigma^{\prime}\right) \delta_{\sigma \sigma^{\prime \prime}} \delta_{\sigma^{\prime} \sigma^{\prime \prime \prime}}
\end{aligned}
$$

For atomic orbitals, whose shapes are independent of the spin, we can write the interaction tensor elements as

$$
U_{m \sigma, m^{\prime} \sigma^{\prime}, m^{\prime \prime} \sigma^{\prime \prime}, m^{\prime \prime \prime} \sigma^{\prime \prime \prime}}=U_{m m^{\prime} m^{\prime \prime} m^{\prime \prime \prime}} \delta_{\sigma \sigma^{\prime \prime}} \delta_{\sigma^{\prime} \sigma^{\prime \prime \prime}}
$$

where

$$
U_{m m^{\prime} m^{\prime \prime} m^{\prime \prime \prime}}=\iint \mathrm{d} \vec{r}_{1} \mathrm{~d} \vec{r}_{2} \phi_{m}^{*}\left(\vec{r}_{1}\right) \phi_{m^{\prime}}^{*}\left(\vec{r}_{2}\right) w\left(\vec{r}_{1}, \vec{r}_{2}\right) \phi_{m^{\prime \prime}}\left(\vec{r}_{1}\right) \phi_{m^{\prime \prime \prime}}\left(\vec{r}_{2}\right) .
$$

In this case the interaction part of eq. (3.22) becomes

$$
\hat{H}_{\mathrm{int}}=\frac{1}{2} \sum_{m m^{\prime} m^{\prime \prime} m^{\prime \prime \prime}} \sum_{\sigma \sigma^{\prime}} U_{m m^{\prime} m^{\prime \prime} m^{\prime \prime \prime}} \hat{c}_{m \sigma}^{\dagger} \hat{c}_{m^{\prime} \sigma^{\prime}}^{\dagger} \hat{c}_{m^{\prime \prime \prime} \sigma^{\prime}} \hat{c}_{m^{\prime \prime} \sigma}
$$

\subsection{The Anderson impurity model}

A popular model Hamiltonian for studies of systems with localized magnetic impurities embedded in a metallic bath is the Anderson impurity model, which has been introduced by P. W. Anderson [58] in 1961. In the context of this thesis, the Anderson impurity model is employed as a simple model system for the rare-earth hexaborides, whose electronic structure is composed of itinerant and localized particles. The localized particles reside in the $4 f$ orbitals of the rare-earth ions.

The full many-body Hamiltonian of the Anderson impurity model [58] consists of three contributions,

$$
\hat{H}=\hat{H}_{\mathrm{bath}}+\hat{H}_{\mathrm{loc}}+\hat{H}_{\mathrm{hyb}} \text {. }
$$

First, the bath of non-interacting particles is described by

$$
\hat{H}_{\text {bath }}=\sum_{\alpha} \varepsilon_{\alpha} \hat{c}_{\alpha}^{\dagger} \hat{c}_{\alpha}
$$

where $\hat{c}_{\alpha}^{\dagger}$ and $\hat{c}_{\alpha}$ denote the annihilation and creation operators for a bath particle with quantum number $\alpha$, respectively. The energy of a one-particle state with quantum number $\alpha$ is $\varepsilon_{\alpha}$. Second, the site of localized orbitals is modeled via a Hamiltonian of the form (3.22),

$$
\hat{H}_{\mathrm{loc}}=\sum_{a b} h_{a b} \hat{f}_{a}^{\dagger} \hat{f}_{b}+\frac{1}{2} \sum_{a b c d} U_{a b d c} \hat{f}_{a}^{\dagger} \hat{f}_{b}^{\dagger} \hat{f}_{c} \hat{f}_{d},
$$

where $h_{a b}$ is an element of the one-particle Hamiltonian matrix and $U_{a b c d}$ is an 
element of the interaction tensor. The operators $\hat{f}_{a}^{\dagger}$ and $\hat{f}_{a}$ create and annihilate a particle in a localized orbital $a$, respectively. Lastly, the bath and the site of localized orbitals are coupled via the hybridization

$$
\hat{H}_{\mathrm{hyb}}=\sum_{\alpha b}\left(V_{\alpha b} \hat{c}_{\alpha}^{\dagger} \hat{f}_{b}+V_{\alpha b}^{*} \hat{f}_{b}^{\dagger} \hat{c}_{\alpha}\right)
$$

where $V_{\alpha b}$ is the hybridization strength.

\section{| The Rayleigh-Ritz principle}

In solid state physics one is often interested in finding the ground state energy, i. e. the lowest energy a system can attain. Techniques, including the HartreeFock method and density functional theory, rely on the so-called Rayleigh-Ritz principle, which is the foundation of the ground state search for eigenvalue problems. It states that the energy corresponding to a state $|\Psi\rangle$ is larger or equal to the ground state energy,

$$
\langle\Psi|\hat{H}| \Psi\rangle \geq E_{0}\langle\Psi \mid \Psi\rangle
$$

where equality holds for $|\Psi\rangle$ being the ground state. Many algorithms for ground state search are hence based on minimization of the energy by variation of the state $|\Psi\rangle$. Usually, this leads to an upper bound for the ground state energy, which, however, often is a good approximation for the real ground state energy.

\subsection{Hartree-Fock theory}

The complicated nature of a many-particle system originates from the twobody interaction. Under the assumption that the interaction can be reasonably well described in terms of a single-particle potential, the state of the manybody system can be approximated as one of a non-interacting Hamiltonian. In this way the potential of this non-interacting Hamiltonian represents an average of the interaction effects which is why such an approach is known as a mean-field approximation. Because the effective Hamiltonian in the meanfield approximation is a non-interacting Hamiltonian, the particles inside the system are essentially regarded as being independent. A caveat of the HartreeFock approximation is that the effective potential representing the interaction depends on the solution of the effective Hamiltonian itself and the mean-field solution to a many-particle problem has to be computed self-consistently.

In this thesis we are concerned with fermionic systems. A many-particle wave function of a fermionic system has to be antisymmetric with respect to an interchange of the coordinates of two fermions. This is known as the Pauli principle and imposes an additional restriction on a system of independent fermions over a system of, e. g., independent classical particles. As a consequence the mean-field approximation for a system of fermions differs from the mean-field 
approximation for classical or bosonic systems. For fermions the mean-field approximation is often referred to as Hartree-Fock approximation. In the following we present a formulation of the Hartree-Fock approximation which is based on the minimization of the grand canonical potential. For convenience we use the framework of second quantization. In the derivation we follow the corresponding text passages in the book of Czycholl [59] (in german).

Because the derivation of the Hartree-Fock approximation is based on the minimization principle of the grand canonical potential, we remind the reader that the density operator for the grand canonical ensemble is given by

$$
\hat{\Gamma}_{\mathrm{GC}}=\frac{1}{Z_{\mathrm{GC}}} e^{-\beta(\hat{H}-\mu \hat{N})} .
$$

Here and in the remainder of this section the Hamiltonian $\hat{H}$ is the Hamiltonian of the interacting system at hand and, in general, is of the form (3.22). The operator $\hat{N}$ is the total particle number operator. Furthermore, we introduced $\beta=1 /\left(k_{\mathrm{B}} T\right)$, where $k_{\mathrm{B}}$ is the Boltzmann constant and $T$ is the temperature. $\mu$ denotes the chemical potential. The grand canonical partition function reads

$$
Z_{\mathrm{GC}}=\operatorname{Tr}\left[e^{-\beta(\hat{H}-\mu \hat{N})}\right]
$$

Moreover, the grand canonical potential is defined by

$$
\Omega_{\mathrm{GC}}=-\frac{1}{\beta} \ln Z_{\mathrm{GC}}
$$

Next, we define

$$
\Omega[\hat{\Gamma}]=\operatorname{Tr}\left[\hat{\Gamma}\left(\hat{H}-\mu \hat{N}+\frac{1}{\beta} \ln \hat{\Gamma}\right)\right]
$$

as a functional of a density operator $\hat{\Gamma}$, for which $\operatorname{Tr} \hat{\Gamma}=1$. The functional eq. (3.40) is central to the derivation of the Hartree-Fock approximation presented here. It has two important properties that we state in the following. First, for the density operator of the grand canonical ensemble, eq. (3.37), the functional (3.40) returns the grand canonical potential:

$$
\begin{aligned}
\Omega\left[\hat{\Gamma}_{\mathrm{GC}}\right] & =\operatorname{Tr}\left[\frac{1}{Z_{\mathrm{GC}}} e^{-\beta(\hat{H}-\mu \hat{N})}\left(\hat{H}-\mu \hat{N}+\frac{1}{\beta}\left\{-\ln Z_{\mathrm{GC}}-\beta(\hat{H}-\mu \hat{N})\right\}\right)\right] \\
& =-\frac{1}{\beta} \ln Z_{\mathrm{GC}} \operatorname{Tr}\left[\frac{1}{Z_{\mathrm{GC}}} e^{-\beta(\hat{H}-\mu \hat{N})}\right] \\
& =-\frac{1}{\beta} \ln Z_{\mathrm{GC}} \\
& =\Omega_{\mathrm{GC}}
\end{aligned}
$$

Second, the functional (3.40) is minimal for the grand canonical density oper- 
ator (3.37),

$$
\Omega[\hat{\Gamma}] \geq \Omega\left[\hat{\Gamma}_{\mathrm{GC}}\right]
$$

This can be proven as follows. For an arbitrary normalized density operator $\hat{\Gamma}$ the inequality (3.45) suggests

$$
\begin{aligned}
\Omega[\hat{\Gamma}] & =\operatorname{Tr}[\hat{\Gamma}(\hat{H}-\mu \hat{N})]+\frac{1}{\beta} \operatorname{Tr}[\hat{\Gamma} \ln \hat{\Gamma}] \\
& \geq \operatorname{Tr}[\hat{\Gamma}(\hat{H}-\mu \hat{N})]+\frac{1}{\beta} \operatorname{Tr}\left[\hat{\Gamma} \ln \hat{\Gamma}_{\mathrm{GC}}\right] \\
& =-\frac{1}{\beta} \ln Z_{\mathrm{GC}} \operatorname{Tr} \hat{\Gamma} \\
& =\Omega_{\mathrm{GC}} .
\end{aligned}
$$

Hence, we need to prove that

$$
\operatorname{Tr}[\hat{\Gamma} \ln \hat{\Gamma}] \geq \operatorname{Tr}\left[\hat{\Gamma} \ln \hat{\Gamma}_{\mathrm{GC}}\right]
$$

To do so, we introduce an index $\alpha$ to label the states of the eigenbasis $\{|\Psi\rangle\}$ of a density operator $\hat{\Gamma}$. Similarly, we use an index $v$ to label the states of the eigenbasis $\left\{\left|\Psi^{\prime}\right\rangle\right\}$ of a second density operator $\hat{\Gamma}^{\prime}$. Then we have

$$
\operatorname{Tr}[\hat{\Gamma} \ln \hat{\Gamma}]=\sum_{\alpha} \Gamma_{\alpha} \ln \Gamma_{\alpha}
$$

and

$$
\begin{aligned}
\operatorname{Tr}\left[\hat{\Gamma} \ln \hat{\Gamma}^{\prime}\right] & =\sum_{v}\left\langle\Psi_{v}^{\prime}|\hat{\Gamma}| \Psi_{v}^{\prime}\right\rangle \ln \Gamma_{v}^{\prime} \\
& =\sum_{\alpha v}\left|\left\langle\Psi_{v}^{\prime} \mid \Psi_{\alpha}\right\rangle\right|^{2} \Gamma_{\alpha} \ln \Gamma_{v}^{\prime}
\end{aligned}
$$

where $\Gamma_{\alpha}$ and $\Gamma_{\nu}^{\prime}$ denote the ensemble weights associated with the states $\left|\Psi_{\alpha}\right\rangle$ and $\left|\Psi_{v}^{\prime}\right\rangle$, respectively. By subtracting eq. (3.51) from eq. (3.53) and exploiting the fundamental inequality $\ln x \leq x-1$, we obtain

$$
\begin{aligned}
\operatorname{Tr}\left[\hat{\Gamma} \ln \hat{\Gamma}^{\prime}\right]-\operatorname{Tr}[\hat{\Gamma} \ln \hat{\Gamma}] & =\sum_{\alpha v} \Gamma_{\alpha}\left|\left\langle\Psi_{v}^{\prime} \mid \Psi_{\alpha}\right\rangle\right|^{2} \ln \frac{\Gamma_{v}^{\prime}}{\Gamma_{\alpha}} \\
& \leq \sum_{\alpha v} \Gamma_{\alpha}\left|\left\langle\Psi_{v}^{\prime} \mid \Psi_{\alpha}\right\rangle\right|^{2}\left(\frac{\Gamma_{v}^{\prime}}{\Gamma_{\alpha}}-1\right) \\
& =\sum_{v} \Gamma_{v}^{\prime}-\sum_{\alpha} \Gamma_{\alpha} \\
& =0 .
\end{aligned}
$$

This result is equivalent to inequality (3.50) and completes the proof of inequality (3.45).

Now we turn our attention to the general idea of the Hartree-Fock approximation, which is to model a system of interacting fermions as best as possible 
by an auxiliary system of non-interacting fermions in an effective potential. Suppose the parameters $h_{a b}$ and $U_{a b c d}$ of the interacting Hamiltonian $\hat{H}$ are known in the orthonormal one-particle basis $\{|\varphi\rangle\}$. The Hamiltonian of the effective non-interacting system in the basis $\{|\varphi\rangle\}$ reads

$$
\hat{H}_{\mathrm{HF}}=\sum_{a b} h_{a b}^{\mathrm{HF}} \hat{c}_{a}^{\dagger} \hat{c}_{b}
$$

where the creation operator $\hat{c}_{a}^{\dagger}$ creates a particle in the orbital $\left|\varphi_{a}\right\rangle$ and the annihilation operator $\hat{c}_{b}$ annihilates a particle in the orbital $\left|\varphi_{b}\right\rangle$. We can also write the Hamiltonian in diagonal form,

$$
\hat{H}_{\mathrm{HF}}=\sum_{i} \xi_{i} \hat{a}_{i}^{\dagger} \hat{a}_{i}
$$

where $\hat{a}_{i}^{\dagger}$ and $\hat{a}_{i}$ denote creation and annihilation operators for a particle in an orbital $\left|\phi_{i}\right\rangle$ of the diagonal orthonormal one-particle basis $\{|\phi\rangle\}$, respectively. A one-particle basis state of either basis can be expanded in terms of the other basis via

$$
\left|\varphi_{a}\right\rangle=\sum_{i} S_{i a}\left|\phi_{i}\right\rangle, \quad \text { and } \quad\left|\phi_{i}\right\rangle=\sum_{i} S_{i a}^{*}\left|\varphi_{a}\right\rangle,
$$

where $S_{i a}=\left\langle\phi_{i} \mid \varphi_{a}\right\rangle$.

The grand canonical density operator associated with the effective Hamiltonian (3.58) is

$$
\hat{\Gamma}_{\mathrm{HF}}=\frac{1}{Z_{\mathrm{HF}}} e^{-\beta\left(\hat{H}_{\mathrm{HF}}-\mu \hat{N}\right)},
$$

where the partition function reads

$$
Z_{\mathrm{HF}}=\operatorname{Tr}\left[e^{-\beta\left(\hat{H}_{\mathrm{HF}}-\mu \hat{N}\right)}\right]
$$

Moreover, we define the grand canonical potential of the auxiliary system via

$$
\Omega_{\mathrm{HF}}=-\frac{1}{\beta} \ln Z_{\mathrm{HF}}
$$

In the preceding paragraphs we saw that, with the Hamiltonian $\hat{H}$ of the system of interacting fermions at hand, the functional (3.40) is minimized by the grand canonical density operator of the form (3.37). Based on this observation, we define that auxiliary system of non-interacting fermions to model the interacting system "as best as possible" which minimizes the functional (3.40) over the set of all density operators of the form (3.61). In the following we derive the matrix $\mathbf{h}^{\mathrm{HF}}$ that defines the non-interacting system we are looking for.

With $\hat{\Gamma}_{\mathrm{HF}}$ as functional variable in eq. (3.40) we obtain

$$
\Omega\left[\hat{\Gamma}_{\mathrm{HF}}\right]=\operatorname{Tr}\left[\hat{\Gamma}_{\mathrm{HF}}\left(\hat{H}-\mu \hat{N}-\hat{H}_{\mathrm{HF}}+\mu \hat{N}-\frac{1}{\beta} \ln Z_{\mathrm{HF}}\right)\right]
$$




$$
\begin{aligned}
& =-\frac{1}{\beta} \ln Z_{\mathrm{HF}}+\operatorname{Tr}\left[\hat{\Gamma}_{\mathrm{HF}} \hat{H}\right]-\operatorname{Tr}\left[\hat{\Gamma}_{\mathrm{HF}} \hat{H}_{\mathrm{HF}}\right] \\
& =\Omega_{\mathrm{HF}}+\langle\hat{H}\rangle_{\mathrm{HF}}-\left\langle\hat{H}_{\mathrm{HF}}\right\rangle_{\mathrm{HF}} .
\end{aligned}
$$

We introduced the notation $\langle\hat{X}\rangle_{\mathrm{HF}}=\operatorname{Tr}\left[\hat{\Gamma}_{\mathrm{HF}} \hat{X}\right]$ for the expectation value of an operator $\hat{X}$ with respect to the density operator (3.61).

For the second term of eq. (3.66), after inserting the interacting Hamiltonian (3.22), we obtain

$$
\langle\hat{H}\rangle_{\mathrm{HF}}=\sum_{a b} h_{a b}\left\langle\hat{c}_{a}^{\dagger} \hat{c}_{b}\right\rangle_{\mathrm{HF}}+\frac{1}{2} \sum_{a b c d} U_{a b c d}\left\langle\hat{c}_{a}^{\dagger} \hat{c}_{b}^{\dagger} \hat{c}_{d} \hat{c}_{c}\right\rangle_{\mathrm{HF}} .
$$

The expectation value of the operator sequence in the interaction part of eq. (3.67) can be written as

$$
\left\langle\hat{c}_{a}^{\dagger} \hat{c}_{b}^{\dagger} \hat{c}_{d} \hat{c}_{c}\right\rangle_{\mathrm{HF}}=\left\langle\hat{c}_{a}^{\dagger} \hat{c}_{c}\right\rangle_{\mathrm{HF}}\left\langle\hat{c}_{b}^{\dagger} \hat{c}_{d}\right\rangle_{\mathrm{HF}}-\left\langle\hat{c}_{a}^{\dagger} \hat{c}_{d}\right\rangle_{\mathrm{HF}}\left\langle\hat{c}_{b}^{\dagger} \hat{c}_{c}\right\rangle_{\mathrm{HF}} .
$$

A proof for eq. (3.68) is given in appendix A. We remark that an expression of the form $\left\langle\hat{c}_{b}^{\dagger} \hat{c}_{a}\right\rangle_{\mathrm{HF}}$ is simply an element of the one-particle density matrix, obtained from the grand canonical ensemble of the effective non-interacting system. After inserting eq. (3.68) into eq. (3.67) and relabeling the indices, the expectation value $\langle\hat{H}\rangle_{\mathrm{HF}}$ becomes

$$
\langle\hat{H}\rangle_{\mathrm{HF}}=\sum_{a b} h_{a b}\left\langle\hat{c}_{a}^{\dagger} \hat{c}_{b}\right\rangle_{\mathrm{HF}}+\frac{1}{2} \sum_{a b c d}\left(U_{d a c b}-U_{d a b c}\right)\left\langle\hat{c}_{a}^{\dagger} \hat{c}_{b}\right\rangle_{\mathrm{HF}}\left\langle\hat{c}_{d}^{\dagger} \hat{c}_{c}\right\rangle_{\mathrm{HF}} .
$$

The third term of eq. (3.66) is the expectation value of the Hamiltonian of the effective non-interacting system with respect to the grand canonical density operator of the effective non-interacting system,

$$
\left\langle\hat{H}_{\mathrm{HF}}\right\rangle_{\mathrm{HF}}=\sum_{a b} h_{a b}^{\mathrm{HF}}\left\langle\hat{c}_{a}^{\dagger} \hat{c}_{b}\right\rangle_{\mathrm{HF}}=\sum_{i} \xi_{i}\left\langle\hat{a}_{i}^{\dagger} \hat{a}_{i}\right\rangle_{\mathrm{HF}}
$$

For a fixed temperature and a fixed chemical potential the density operator $\hat{\Gamma}_{\mathrm{HF}}$ depends only on the matrix $\mathbf{h}^{\mathrm{HF}}$ as a variable. We minimize the functional (3.66) with respect to the matrix $\mathbf{h}^{\mathrm{HF}}$ :

$$
\begin{aligned}
\frac{\partial \Omega\left[\hat{\Gamma}_{\mathrm{HF}}\right]}{\partial h_{m n}^{\mathrm{HF}}}=\frac{\partial \Omega_{\mathrm{HF}}^{\mathrm{HF}}}{\partial h_{m n}^{\mathrm{HF}}}+\sum_{a b} h_{a b} \frac{\partial\left\langle\hat{c}_{a}^{\dagger} \hat{c}_{b}\right\rangle_{\mathrm{HF}}}{\partial h_{m n}^{\mathrm{HF}}}+\frac{1}{2} \sum_{a b c d}\left(U_{d a c b}-U_{d a b c}\right) \times \\
\quad \times\left(\left\langle\hat{c}_{a}^{\dagger} \hat{c}_{b}\right\rangle_{\mathrm{HF}} \frac{\partial\left\langle\hat{c}_{d}^{\dagger} \hat{c}_{c}\right\rangle_{\mathrm{HF}}}{\partial h_{m n}^{\mathrm{HF}}}+\left\langle\hat{c}_{d}^{\dagger} \hat{c}_{c}\right\rangle_{\mathrm{HF}} \frac{\partial\left\langle\hat{c}_{a}^{\dagger} \hat{c}_{b}\right\rangle_{\mathrm{HF}}}{\partial h_{m n}^{\mathrm{HF}}}\right) \\
-\left\langle\hat{c}_{m}^{\dagger} \hat{c}_{n}\right\rangle_{\mathrm{HF}}-\sum_{a b} h_{a b}^{\mathrm{HF}} \frac{\partial\left\langle\hat{c}_{a}^{\dagger} \hat{c}_{b}\right\rangle_{\mathrm{HF}}}{\partial h_{m n}^{\mathrm{HF}}} .
\end{aligned}
$$

This can be simplified by a relabeling of indices in the third term and by 
exploiting the symmetry of the interaction tensor, $U_{a b c d}=U_{b a d c}$. We arrive at

$$
\begin{aligned}
\frac{\partial \Omega\left[\hat{\Gamma}_{\mathrm{HF}}\right]}{\partial h_{m n}^{\mathrm{HF}}}= & \frac{\partial \Omega_{\mathrm{HF}}^{\mathrm{HF}}}{\partial h_{m n}^{\mathrm{HF}}}-\left\langle\hat{c}_{m}^{\dagger} \hat{c}_{n}\right\rangle_{\mathrm{HF}} \\
& +\sum_{a b}\left[h_{a b}+\sum_{c d}\left(U_{d a c b}-U_{d a b c}\right)\left\langle\hat{c}_{d}^{\dagger} \hat{c}_{c}\right\rangle_{\mathrm{HF}}-h_{a b}^{\mathrm{HF}}\right] \frac{\partial\left\langle\hat{c}_{a}^{\dagger} \hat{c}_{b}\right\rangle_{\mathrm{HF}}}{\partial h_{m n}^{\mathrm{HF}}}
\end{aligned}
$$

Next, we evaluate the first term of eq. (3.72):

$$
\begin{aligned}
\frac{\partial \Omega_{\mathrm{HF}}}{\partial h_{m n}^{\mathrm{HF}}} & =-\frac{1}{\beta} \frac{\partial}{\partial h_{m n}^{\mathrm{HF}}} \ln Z_{\mathrm{HF}} \\
& =-\frac{1}{\beta} \frac{1}{Z_{\mathrm{HF}}} \frac{\partial}{\partial h_{m n}^{\mathrm{HF}}} \operatorname{Tr}\left[e^{-\beta \sum_{i}\left(\xi_{i}-\mu\right) \hat{a}_{i}^{\dagger} \hat{a}_{i}}\right] \\
& =\sum_{i}\left\langle\hat{a}_{i}^{\dagger} \hat{a}_{i}\right\rangle_{\mathrm{HF}} \frac{\partial \xi_{i}}{\partial h_{m n}^{\mathrm{HF}}} \\
& =\left\langle\hat{c}_{m}^{\dagger} \hat{c}_{n}\right\rangle_{\mathrm{HF}},
\end{aligned}
$$

where in the last step the basis transformation formula for the eigenvalues, $\xi_{i}=\sum_{a b} S_{a i}^{*} S_{b i} h_{a b}^{\mathrm{HF}}$, was exploited. Hence, eq. (3.72) simplifies to

$$
\frac{\partial \Omega\left[\hat{\Gamma}_{\mathrm{HF}}\right]}{\partial h_{m n}^{\mathrm{HF}}}=\sum_{a b}\left[h_{a b}+\sum_{c d}\left(U_{d a c b}-U_{d a b c}\right)\left\langle\hat{c}_{d}^{\dagger} \hat{c}_{c}\right\rangle_{\mathrm{HF}}-h_{a b}^{\mathrm{HF}}\right] \frac{\partial\left\langle\hat{c}_{a}^{\dagger} \hat{c}_{b}\right\rangle_{\mathrm{HF}}}{\partial h_{m n}^{\mathrm{HF}}}
$$

We see that the minimum condition $\partial \Omega\left[\hat{\Gamma}_{\mathrm{HF}}\right] / \partial h_{m n}^{\mathrm{HF}}=0$ is satisfied by

$$
h_{a b}^{\mathrm{HF}}=h_{a b}+\sum_{c d}\left(U_{d a c b}-U_{d a b c}\right)\left\langle\hat{c}_{d}^{\dagger} \hat{c}_{c}\right\rangle_{\mathrm{HF}} \cdot
$$

This means that the "best approximation" of an interacting Hamiltonian (3.22) in terms of an effective non-interacting Hamiltonian (3.58) is obtained, if the matrix elements $h_{a b}^{\mathrm{HF}}$ are of the form (3.78). "Best approximation" is to be understood in the sense that the value of the functional (3.40) for a density operator (3.61) is minimal with respect to variations of the non-interacting Hamiltonian (3.58). This minimum is as as close as possible to the true minimum of the functional (3.40), which is found for the grand canonical density operator (3.37), where $\hat{H}$ is the interacting Hamiltonian of the system at hand.

Note that the matrix elements (3.78) depend on the expectation value $\left\langle\hat{c}_{d}^{\dagger} \hat{c}_{c}\right\rangle_{\mathrm{HF}}$, for whose calculation typically the matrix elements (3.78) need to be known. This dependency circle can be resolved in a self-consistent calculation. Because we do not need to employ self-consistency algorithms in the context of this thesis we do not pursue this broad topic any further.

After inserting eq. (3.78) into eq. (3.58) we see that the Hamiltonian of the 
effective non-interacting system can be written as

$$
\hat{H}_{\mathrm{HF}}=\hat{H}_{0}+\hat{V}_{\mathrm{H}}+\hat{V}_{\mathrm{X}}
$$

where

$$
\hat{H}_{0}=\sum_{a b} h_{a b} \hat{c}_{a}^{\dagger} \hat{c}_{b}
$$

is the non-interacting part of the original interacting Hamiltonian,

$$
\hat{V}_{\mathrm{H}}=\sum_{a b c d} U_{d a c b}\left\langle\hat{c}_{d}^{\dagger} \hat{c}_{c}\right\rangle_{\mathrm{HF}} \hat{c}_{a}^{\dagger} \hat{c}_{b}
$$

is the Hartree potential, and

$$
\hat{V}_{\mathrm{X}}=-\sum_{a b c d} U_{d a b c}\left\langle\hat{c}_{d}^{\dagger} \hat{c}_{c}\right\rangle_{\mathrm{HF}} \hat{c}_{a}^{\dagger} \hat{c}_{b}
$$

is the exchange potential. With the effective potential operators (3.81) and (3.82) we obtain the Hartree energy

$$
E_{\mathrm{H}}=\frac{1}{2}\left\langle\hat{V}_{\mathrm{H}}\right\rangle_{\mathrm{HF}}=\frac{1}{2} \sum_{a b c d} U_{d a c b}\left\langle\hat{c}_{a}^{\dagger} \hat{c}_{b}\right\rangle_{\mathrm{HF}}\left\langle\hat{c}_{d}^{\dagger} \hat{c}_{c}\right\rangle_{\mathrm{HF}}
$$

and the exchange energy

$$
E_{\mathrm{X}}=\frac{1}{2}\left\langle\hat{V}_{\mathrm{X}}\right\rangle_{\mathrm{HF}}=-\frac{1}{2} \sum_{a b c d} U_{d a b c}\left\langle\hat{c}_{a}^{\dagger} \hat{c}_{b}\right\rangle_{\mathrm{HF}}\left\langle\hat{c}_{d}^{\dagger} \hat{c}_{c}\right\rangle_{\mathrm{HF}},
$$

respectively.

It is important to note that the grand canonical potential $\Omega_{\mathrm{HF}}$ with respect to the effective non-interacting Hamiltonian differs from the value $\Omega\left[\hat{\Gamma}_{\mathrm{HF}}\right]$. According to eq. (3.66) the difference $\Omega\left[\hat{\Gamma}_{\mathrm{HF}}\right]-\Omega_{\mathrm{HF}}$ is equal to the difference $\langle\hat{H}\rangle_{\mathrm{HF}}-\left\langle\hat{H}_{\mathrm{HF}}\right\rangle_{\mathrm{HF}}$ of the expectation values of the interacting Hamiltonian $\hat{H}$ and the effective non-interacting Hamiltonian $\hat{H}_{\mathrm{HF}}$ with respect to the density operator $\hat{\Gamma}_{\mathrm{HF}}$. To obtain an expression for this difference we use eq. (3.69), and eq. (3.70) together with eq. (3.78), and find

$$
\begin{aligned}
\Omega\left[\hat{\Gamma}_{\mathrm{HF}}\right]-\Omega_{\mathrm{HF}} & =\langle\hat{H}\rangle_{\mathrm{HF}}-\left\langle\hat{H}_{\mathrm{HF}}\right\rangle_{\mathrm{HF}} \\
& =-\frac{1}{2} \sum_{a b c d}\left(U_{d a c b}-U_{d a b c}\right)\left\langle\hat{c}_{a}^{\dagger} \hat{c}_{b}\right\rangle_{\mathrm{HF}}\left\langle\hat{c}_{d}^{\dagger} \hat{c}_{c}\right\rangle_{\mathrm{HF}} \\
& =-\left(E_{\mathrm{H}}+E_{\mathrm{X}}\right) .
\end{aligned}
$$

The expectation value $\langle\hat{H}\rangle_{\mathrm{HF}}$ can be used as an approximation to the true total energy $\langle\hat{H}\rangle_{\mathrm{GC}}$ of the interacting system. If $\langle\hat{H}\rangle_{\mathrm{HF}}$ is to be computed from the solution of the effective non-interacting system, it is important to subtract the Hartree and exchange energies from $\left\langle\hat{H}_{\mathrm{HF}}\right\rangle_{\mathrm{HF}}$ since the interaction energy will 
be counted twice otherwise:

$$
\langle\hat{H}\rangle_{\mathrm{GC}} \approx\langle\hat{H}\rangle_{\mathrm{HF}}=\left\langle\hat{H}_{\mathrm{HF}}\right\rangle_{\mathrm{HF}}-\left(E_{\mathrm{H}}+E_{\mathrm{X}}\right) .
$$

\subsubsection{The Hartree-Fock approximation of the Anderson impu- rity model}

In the forgoing section we showed the derivation of the Hartree-Fock approximation of an interacting Hamiltonian of the general form eq. (3.22). In the course of this thesis we will be interested in a simple model system, where an interacting atomic site is coupled to a non-interacting bath via particle exchange. In this situation the interacting site to which the Hartree-Fock approximation shall be applied is an open system. A similar situation occurs in an Anderson impurity model, see section 3.5, where an interaction is present only between a subset of orbitals of the complete system. Here, we briefly discuss the HartreeFock approximation to an open interacting subsystem at the example of the Anderson impurity model.

To apply the Hartree-Fock approximation to the Anderson impurity model, we write the Hamiltonian (3.32) of the Anderson impurity model in the general form

$$
\hat{H}=\sum_{v \mu} h_{v \mu} \hat{a}_{v}^{\dagger} \hat{a}_{\mu}+\frac{1}{2} \sum_{\nu \mu \lambda \kappa} U_{v \mu \kappa \lambda} \hat{a}_{\nu}^{\dagger} \hat{a}_{\mu}^{\dagger} \hat{a}_{\lambda} \hat{a}_{\kappa} .
$$

The indices $v \mu \kappa \lambda$ are general labels for all orbitals within the system and therefore run over the bath states and the localized orbitals. From section 3.7 we know that the effective non-interacting Hamiltonian of the Hartree-Fock approximation takes the form

$$
\begin{aligned}
\hat{H}_{\mathrm{HF}} & =\sum_{v \mu} h_{v \mu}^{\mathrm{HF}} \hat{a}_{\nu}^{\dagger} \hat{a}_{\mu} \\
& =\sum_{v \mu} h_{\nu \mu} \hat{a}_{\nu}^{\dagger} \hat{a}_{\mu}+\sum_{\nu \mu \lambda \kappa}\left(U_{\lambda v \kappa \mu}-U_{\lambda v \mu \kappa}\right)\left\langle\hat{a}_{\lambda}^{\dagger} \hat{a}_{\kappa}\right\rangle_{\mathrm{HF}} \hat{a}_{\nu}^{\dagger} \hat{a}_{\mu} .
\end{aligned}
$$

We split the sums over the general quantum numbers $v \mu \kappa \lambda$ into sums over the bath states and the localized states. Furthermore, we exploit that the interaction is non-zero only between localized states. This gives

$$
\begin{aligned}
\hat{H}_{\mathrm{HF}}=\sum_{\alpha} & \varepsilon_{\alpha} \hat{c}_{\alpha}^{\dagger} \hat{c}_{\alpha}+\sum_{a b} h_{a b} \hat{f}_{a}^{\dagger} \hat{f}_{b}+\sum_{a b c d}\left(U_{d a c b}-U_{d a b c}\right)\left\langle\hat{f}_{d}^{\dagger} \hat{f}_{c}\right\rangle_{\mathrm{HF}} \hat{f}_{a}^{\dagger} \hat{f}_{b} \\
& +\sum_{\alpha b}\left(V_{\alpha b} \hat{c}_{\alpha}^{\dagger} \hat{f}_{b}+V_{\alpha b}^{*} \hat{f}_{b}^{\dagger} \hat{c}_{\alpha}\right) .
\end{aligned}
$$

Hence, clearly also the effective non-interacting Hamiltonian $\hat{H}_{\mathrm{HF}}$ of the Anderson impurity model is of the form (3.79). I. e. we have a non-interacting part $\hat{H}_{0}$ which contains all non-interacting contributions of the Hamiltonian $\hat{H}$. 
Moreover, the Hartree potential

$$
\hat{V}_{\mathrm{H}}=\sum_{a b c d} U_{d a c b}\left\langle\hat{f}_{d}^{\dagger} \hat{f}_{c}\right\rangle_{\mathrm{HF}} \hat{f}_{a}^{\dagger} \hat{f}_{b}
$$

and the exchange potential

$$
\hat{V}_{\mathrm{X}}=-\sum_{a b c d} U_{d a b c}\left\langle\hat{f}_{d}^{\dagger} \hat{f}_{c}\right\rangle_{\mathrm{HF}} \hat{f}_{a}^{\dagger} \hat{f}_{b}
$$

receive contributions from the interaction between the localized orbitals only and they only act on localized orbitals. 


\subsection{Density-functional theory}

In physical and chemical studies of a material, knowledge of its electronic structure is fundamental in order to understand its behavior on both, microscopic and macroscopic scales. Various properties of a material, like the crystal structure, electronic conductivity, magnetic ordering, and optical features of a solid state material, as well as bonding properties of molecules are closely linked to the material's electronic structure. In most common theoretical approaches, information about the electronic structure is extracted from the system's wave function. However, real materials often contain so many electronic degrees of freedom that the calculation of the materials' many-particle wave functions by solving the Schrödinger equation of the electronic Hamiltonian (3.19) is an intractable problem. This is because the computational complexity grows exponentially with increasing system size. In the framework of density-functional theory (DFT), the computational effort is reduced considerably by replacing the many-electron wave function with the electron density as the fundamental variable. With DFT, simulations of many complex materials become feasible with reasonable computational effort. Hence, DFT is probably the most important computational method in the determination of real materials' electronic structures to date. The purpose of the upcoming sections is to give an overview on the theoretical foundations of DFT.

\subsubsection{The Hohenberg-Kohn-theorem}

One of the major breakthroughs in the simulation of materials is the HohenbergKohn-theorem first published in 1964, which is one of the foundations of density functional theory. It states [60]:

Let $n_{0}(\vec{r})$ be the electron charge density of the ground state of a system of interacting electrons in an external potential $v_{\text {ext }}(\vec{r})$, and let the same hold for another pair of electron charge density $n_{0}^{\prime}(\vec{r})$ and external potential $v_{\text {ext }}^{\prime}(\vec{r})$. If the densities are equal, $n_{0}(\vec{r})=$ $n_{0}^{\prime}(\vec{r})$, then the potentials coincide up to an additive constant, $v_{\mathrm{ext}}(\vec{r})=v_{\mathrm{ext}}^{\prime}(\vec{r})+$ const.

This means that the external potential is, up to a constant, uniquely determined by the ground state electron density. Because the external potential is the only system-specific quantity in the electronic Hamiltonian (3.19), the Hamiltonian, the ground state wave function as well as the wave functions of excited states and all observables are determined by the ground state electron density. While the original formulation of the corresponding proof is restricted to the case of a non-degenerate ground state, it was later shown that the theorem also holds in the case of a degenerate ground state (see e.g. [61]).

The Hohenberg-Kohn theorem implies that it is in principle possible to avoid the calculation of observables via the many particle wave function, which can be sketched as

$$
v_{\text {ext }} \stackrel{\text { Schrödinger eqn. }}{\longrightarrow}|\Psi\rangle \longrightarrow \text { observables. }
$$


Instead, for a system of $N$ particles, observables can be computed from the ground state density. In such an approach the ground state density replaces the $N$-particle wave function as the central variable. Since the latter depends on $3 N$ coordinates whereas the former depends on only 3 coordinates, this reduces the computational complexity significantly.

Hohenberg and Kohn also proposed a variational scheme for determining the ground state density $[60,61]$, which is often called the HK variational principle or the second HK theorem. The idea is to set up a total energy functional $E\left[v_{\mathrm{ext}}, n\right]$ and search for the energy minimum with respect to the density $n$. The density that minimizes the energy then is the ground state density $n_{0}$. For the energy minimization, the densities are selected from a set $\mathcal{A}_{N}$, which contains all densities that correspond to an $N$-particle ground state,

$$
\mathcal{A}_{N}=\{n \mid n \text { corresponds to an } N \text {-particle ground state }\} \text {. }
$$

Unfortunately, from the three contributions to the total energy of an interacting electronic system with the Hamiltonian (3.19), namely kinetic energy, potential energy, and interaction energy, only the expression for the potential energy in terms of the density is known explicitly,

$$
\langle\Psi|\hat{V}| \Psi\rangle=\int \mathrm{d} \vec{r} n(\vec{r}) v_{\text {ext }}(\vec{r}) .
$$

The kinetic energy and interaction energy are combined into the HohenbergKohn (HK) functional, which can formally be defined as ${ }^{1}$

$$
F_{\mathrm{HK}}[n]=E\left[v_{\mathrm{ext}}[n]\right]-\int \mathrm{d} \vec{r} v_{\mathrm{ext}}[n] n, \quad \text { where } n \in \mathcal{A}_{N}
$$

Because the kinetic energy as well as the interaction energy do not depend on the details of a specific system, neither does the HK functional (3.97). Hence, the HK functional (3.97) is a universal functional. With eq. (3.97), the ground state energy can be expressed as

$$
E_{\mathrm{GS}}\left[v_{\mathrm{ext}}\right]=\min _{n \in \mathcal{A}_{N}}\left[F_{\mathrm{HK}}[n]+\int \mathrm{d} \vec{r} v_{\mathrm{ext}}(\vec{r}) n(\vec{r})\right] .
$$

In eq. (3.98), $v_{\text {ext }}$ is picked from the set of external potentials $\mathcal{V}_{N}$, which is comprised of all external potentials $v_{\text {ext }}^{\prime}$ for which an $N$-particle system,

\footnotetext{
${ }^{1}$ In the text, I follow the work of Lieb [62] and the book of Eschrig [61], who provide a more general formulation of the original idea of Hohenberg and Kohn. For example the restriction to non-degenerate ground states is dropped. The original Hohenberg Kohn functional reads [60]

$$
F_{\mathrm{HK}}[n]=\left\langle\Psi_{0}[n]|\hat{T}+\hat{U}| \Psi_{0}[n]\right\rangle,
$$

and is only defined for densities that belong to a non-degenerate ground state. Also the functional minimization as in eq. (3.98) has to be performed over this set of densities, instead of the set $\mathcal{A}_{N}$. The density that minimizes the energy then is the ground state density.
} 
described by the Hamiltonian $\hat{H}_{v_{\mathrm{ext}}^{\prime}}$, has a ground state,

$$
\mathcal{V}_{N}=\left\{v_{\mathrm{ext}} \mid \hat{H}_{v_{\mathrm{ext}}} \text { has a ground state }\right\} \text {. }
$$

The energy (3.98) is minimized by the density that corresponds to the ground state of a system with external potential $v_{\text {ext }}$.

There are major problems for the computation of the ground state density in this fashion because the functional $F_{\mathrm{HK}}[n]$ is not known explicitly and neither are the sets $\mathcal{A}_{N}$ and $\mathcal{V}_{N}$ [61]. The restrictions on the density $n \in \mathcal{A}_{N}$ are often formulated by saying that the density is required to be $N$-representable as well as $v$-representable. A density is $N$-representable, if the density can be expressed in terms of an $N$-particle state. Moreover, a density $n$ is $v$-representable, if there exists an external potential that produces an $N$-particle ground state with the corresponding density $n$. Fortunately, it was shown by T. L. Gilbert [63] and later also by J. E. Harriman [64] that any finite non-negative differentiable function is $N$-representable. However, the set of $v$-representable densities is unknown.

In order to overcome the limitation of the Hohenberg-Kohn approach, M. Levy and E. H. Lieb extended the work of Hohenberg and Kohn by introducing new functionals as replacements for the functional $F_{\mathrm{HK}}$. These functionals also work for a larger set of trial densities than $\mathcal{A}_{N}$ and resolve the requirement of $v$-representability.

\subsubsection{Levy's constrained search functional}

In his approach to the theory of density functionals, Levy extends Percus's definition of the kinetic energy functional of a non-interacting electron system [65] to the case of interacting electrons [66]. The basic idea is to collect all wave functions that produce a certain fixed density. From all these wave functions, the one which minimizes the sum of the kinetic energy and the electron-electron interaction is selected. The resulting energy functional reads

$$
F_{\text {Levy }}[n]=\min _{|\Psi\rangle: n=\langle\Psi|\hat{n}| \Psi\rangle}\langle\Psi|(\hat{T}+\hat{U})| \Psi\rangle .
$$

and can be used as a replacement for the Hohenberg-Kohn functional $F_{\mathrm{HK}}[n]$. Since not only ground state wave functions are taken into account, the functional $F_{\text {Levy }}[n]$ is defined for all densities that belong to an $N$-particle wave function, and the minimization

$$
E_{\mathrm{GS}}\left[v_{\mathrm{ext}}\right]=\min _{n \in \mathcal{I}_{N}}\left[F_{\mathrm{Levy}}[n]+\int \mathrm{d} \vec{r} v_{\mathrm{ext}}(\vec{r}) n(\vec{r})\right] .
$$

can be performed over the set

$$
\mathcal{I}_{N}=\{n \mid n \text { is } N \text {-representable }\} \text {, }
$$

instead of $\mathcal{A}_{N}$. This is a valuable improvement, since the set $\mathcal{I}_{N}$ is known from the works of T. L. Gilbert [63] and also J. E. Harriman [64], who showed that any 
finite non-negative differentiable function is $N$-representable. Furthermore, $\mathcal{I}_{N}$ is a convex set, i. e. for $n_{1}, n_{2} \in \mathcal{I}_{N}$ and $\lambda \in \mathbb{R}, 0 \leq \lambda \leq 1, \lambda n_{1}+(1-\lambda) n_{2} \in \mathcal{I}_{N}$, which is desirable in the context of the variational principle. As discussed by Lieb [62], the functional $F_{\text {Levy }}[n]$, however, is not convex, i. e. the convexity condition $F\left[\lambda n_{1}+(1-\lambda) n_{2}\right] \leq \lambda F\left[n_{1}\right]+(1-\lambda) F\left[n_{2}\right]$ is violated. Convexity of the functional is desirable, because in this case the energy-minimizing density is clearly unique. Thus, although an improvement over the original HK functional and sufficient for practical purposes, the Levy functional still has some shortcomings. Lieb, in his work, uses convex-conjugate analysis and the Legendre-Fenchel transformation to further improve the functional. The interested reader is referred to the original publication [62].

\subsubsection{Lieb's ensemble state density functional}

The Hohenberg-Kohn functional and the Levy functional are by construction restricted to pure-state densities. In his extensive analysis of the universal density functional $F[n]$ Lieb [62] also proposed another functional, which searches the density $n$ in an ensemble of many-particle wave functions. The density for a mixed many-body state is

$$
n(\vec{r})=\sum_{i} w_{i} n_{i}(\vec{r})
$$

where $i$ labels the pure states that contribute to the mixed state. The weight associated to the $i$-th pure state is denoted $w_{i}$ and the density coming from the $i$-th pure state is

$$
n_{i}(\vec{r})=\left\langle\Psi_{i}|\hat{n}(\vec{r})| \Psi_{i}\right\rangle .
$$

With eq. (3.104) it is obvious that $n_{i} \in \mathcal{I}_{N}$. Furthermore, the ensemble weights, as usual, have to obey

$$
w_{i} \geq 0 \quad \text { and } \quad \sum_{i} w_{i}=1,
$$

which also implies $w_{i} \leq 1$. The new functional, which we refer to as Lieb's functional, is defined as

$$
F_{\text {Lieb }}[n]=\min _{\{w\},\{|\Psi\rangle\}:\left\langle\Psi_{i}|\hat{n}| \Psi_{i}\right\rangle=n_{i} \in \mathcal{I}_{N}, w_{i} \geq 0, \sum_{i} w_{i}=1, \sum_{i} w_{i} n_{i}=n} \sum_{i} w_{i}\left\langle\Psi_{i}|(\hat{T}+\hat{U})| \Psi_{i}\right\rangle .
$$

Because $n_{i} \in \mathcal{I}_{N}$ and eq. (3.103) is linear in $n_{i}$, also $n \in \mathcal{I}_{N}$. The ground state energy in analogy to eq. (3.101) reads

$$
E_{\mathrm{GS}}\left[v_{\mathrm{ext}}\right]=\min _{n \in \mathcal{I}_{N}}\left[F_{\mathrm{Lieb}}[n]+\int \mathrm{d} \vec{r} v_{\mathrm{ext}}(\vec{r}) n(\vec{r})\right] .
$$

In the case of a pure-ground state density we have $F_{\text {Lieb }}[n]=F_{\text {Levy }}[n]$. More generally, $F_{\text {Lieb }}[n] \leq F_{\text {Levy }}[n]$ because the Lieb functional searches the minimum of kinetic and interaction energy with respect to the density over all 
possible mixed states and is hence minimized over a larger set of densities than the Levy functional. By combining these arguments and the linearity of eq. (3.103), the Lieb functional is shown to be convex.

One of the important advantages of the Lieb functional of the HohenbergKohn functional and the Levy functional is that densities of mixed manyparticle states, as opposed to only pure-state densities, are considered in the energy minimization. This is especially important for computations on metallic materials, where a degeneracy of the ground state can occur.

\subsubsection{The adiabatic connection of non-interacting and interact- ing system}

Just one year after the paper by Hohenberg and Kohn, Kohn and Sham [67] introduced their well known approach to density functional theory. The KohnSham approach leads to a computation scheme for practical simulations of materials, which we present in section 3.8.6 and which is widely used nowadays. In this section, we present the adiabatic connection of a non-interacting and an interacting system, which can be used to provide a foundation for the KohnSham approach.

Before we continue, let us briefly introduce a commonly used notation for the electron-electron interaction energy. The electron-electron interaction energy can be expressed in terms of the two-particle density and is commonly split into the Hartree energy $E_{\mathrm{H}}$ and the exchange-correlation potential $U_{\mathrm{xc}}$ using eq. (3.3):

$$
\begin{aligned}
\langle\Psi|\hat{W}| \Psi\rangle= & \frac{1}{2} \int \mathrm{d} \vec{r}_{1} \int \mathrm{d} \vec{r}_{2} n^{(2)}\left(\vec{r}_{1}, \vec{r}_{2}\right) w\left(\vec{r}_{1}, \vec{r}_{2}\right) \\
= & \underbrace{\frac{1}{2} \int \mathrm{d} \vec{r}_{1} \int \mathrm{d} \vec{r}_{2} n^{(1)}\left(\vec{r}_{1}\right) n^{(1)}\left(\vec{r}_{2}\right) w\left(\vec{r}_{1}, \vec{r}_{2}\right)}_{E_{\mathrm{H}}} \\
& +\underbrace{\frac{1}{2} \int \mathrm{d} \vec{r}_{1} \int \mathrm{d} \vec{r}_{2} n^{(1)}\left(\vec{r}_{1}\right) h\left(\vec{r}_{1}, \vec{r}_{2}\right) w\left(\vec{r}_{1}, \vec{r}_{2}\right)}_{U_{\mathrm{xc}}} .
\end{aligned}
$$

The idea of Kohn and Sham is to replace the kinetic energy part $T$ of the interacting many-particle system within the functional $F[n]$ with the kinetic energy of non-interacting particles $T_{s}[n]$ and choose the single-particle wave functions in such a way that the density of the non-interacting system is equal to the density of the interacting system. The difference in the kinetic energy of interacting and non-interacting system is not ignored but integrated into the exchange-correlation part of the functional $F[n], E_{\mathrm{xc}}=U_{\mathrm{xc}}+T-T_{s}$. Then, the functional $F[n]$ can be expressed as (see e.g. $[68,69]$ )

$$
F[n]=T_{s}[n]+E_{\mathrm{H}}[n]+E_{\mathrm{xc}}[n] .
$$

Equation (3.110) is a definition of the exchange-correlation energy functional 
$E_{\mathrm{xc}}[n]$ in terms of $F[n], T_{s}[n]$, and $E_{\mathrm{H}}[n]$, which are known to be functionals of the density.

The exchange correlation energy can be obtained by the adiabatic connection of the eigenstates of a system of non-interacting particles to the eigenstates of a system of interacting particles by slowly turning on an interaction while keeping the density constant [70]. In the following, the adiabatic connection is shown, based on a formulation in terms of functionals.

First, let $\hat{H}_{\lambda}=\hat{H}_{0}+\lambda \hat{W}, \lambda \in[0,1]$ be the Hamiltonian describing the adiabatic transition from a non-interacting system with Hamiltonian $\hat{H}_{0}$ to a fully interacting system with Hamiltonian $\hat{H}_{0}+\hat{W}$ by slowly increasing the value of the parameter $\lambda$. The functional for the energy then reads

$$
E_{\lambda}[n]=\left\langle\Psi_{\lambda}\left|\hat{H}_{\lambda}\right| \Psi_{\lambda}\right\rangle=\left\langle\Psi_{\lambda}\left|\hat{H}_{0}\right| \Psi_{\lambda}\right\rangle+\lambda\left\langle\Psi_{\lambda}|\hat{W}| \Psi_{\lambda}\right\rangle .
$$

Since in the Kohn-Sham approach the electron density of the non-interacting replacement system shall be equal to the density of the interacting system, a Lagrange multiplier $v_{\mathrm{eff}, \lambda}$ is included for this constraint. In addition, another Lagrange multiplier $\Lambda_{\lambda}$ is introduced for the normalization of $\left|\Psi_{\lambda}\right\rangle$. The total functional reads

$$
\begin{aligned}
\tilde{E}_{\lambda}[n]=\left\langle\Psi_{\lambda}\left|\hat{H}_{0}\right| \Psi_{\lambda}\right\rangle+\lambda\left\langle\Psi_{\lambda}|\hat{W}| \Psi_{\lambda}\right\rangle \\
\quad+\int \mathrm{d} \vec{r} v_{\text {eff }, \lambda}\left[\left\langle\Psi_{\lambda}|\hat{n}| \Psi_{\lambda}\right\rangle-n(\vec{r})\right]+\Lambda_{\lambda}\left[\left\langle\Psi_{\lambda} \mid \Psi_{\lambda}\right\rangle-1\right]
\end{aligned}
$$

In order to find the ground state energy, this functional shall be minimized with respect to the wave function, subject to the stationary conditions due to the constraints. The resulting functional derivative is

$$
\frac{\delta \tilde{E}_{\lambda}}{\delta\left\langle\Psi_{\lambda}\right|}=\left[\hat{H}_{0}+\lambda \hat{W}+\int \mathrm{d} \vec{r} v_{\mathrm{eff}, \lambda} \hat{n}+\Lambda_{\lambda}\right]\left|\Psi_{\lambda}\right\rangle \stackrel{!}{=} 0
$$

Furthermore, exploiting the constraints $\left\langle\Psi_{\lambda}|\hat{n}(\vec{r})| \Psi_{\lambda}\right\rangle=n(\vec{r})$ and $\left\langle\Psi_{\lambda} \mid \Psi_{\lambda}\right\rangle=1$, as well as eq. (3.113), a straightforward calculation yields

$$
\frac{\partial \tilde{E}_{\lambda}}{\partial \lambda}=\left\langle\Psi_{\lambda}|\hat{W}| \Psi_{\lambda}\right\rangle
$$

Thus, employing eq. (3.109), the ground state energy difference between the interacting and the non-interacting system can be expressed by

$$
\begin{aligned}
E_{1}-E_{0} & =\int_{0}^{1} \mathrm{~d} \lambda \frac{\partial \tilde{E}_{\lambda}}{\partial \lambda}=\int_{0}^{1} \mathrm{~d} \lambda\left\langle\Psi_{\lambda}|\hat{W}| \Psi_{\lambda}\right\rangle \\
& =E_{\mathrm{H}}+\frac{1}{2} \int \mathrm{d} \vec{r} \int \mathrm{d} \vec{r}^{\prime} n(\vec{r}) w\left(\vec{r}-\vec{r}^{\prime}\right) \int_{0}^{1} \mathrm{~d} \lambda h_{\lambda}\left(\vec{r}, \vec{r}^{\prime}\right) .
\end{aligned}
$$

Turning on the interaction adds additional Hartree and exchange-correlation 
parts to the total energy. Because the density is kept fixed, the Hartreeenergy is invariant with respect to $\lambda$ and a change in $\lambda$ can only affect the exchange-correlation part through the hole function. Explicitly, instead of the hole function as in the exchange-correlation potential $U_{\mathrm{xc}}$, the second part of eq. (3.116) exhibits an integrated hole function,

$$
\bar{h}_{\mathrm{xc}}\left(\vec{r}, \vec{r}^{\prime}\right)=\int_{0}^{1} \mathrm{~d} \lambda h_{\lambda}\left(\vec{r}, \vec{r}^{\prime}\right),
$$

and is the exchange-correlation energy

$$
E_{\mathrm{xc}}[n]=\frac{1}{2} \int \mathrm{d} \vec{r} \int \mathrm{d} \vec{r}^{\prime} n(\vec{r}) w\left(\vec{r}-\vec{r}^{\prime}\right) \bar{h}_{\mathrm{xc}}\left(\vec{r}, \vec{r}^{\prime}\right)
$$

Note that $E_{\mathrm{xc}}[n]$ has an implicit dependence on the density through the hole function $\bar{h}_{\mathrm{xc}}\left(\vec{r}, \vec{r}^{\prime}\right)$.

In summary, a system of non-interacting electrons is connected to a system of interacting electrons of the same density by following a unique path through state space while the interaction is adiabatically turned on. A potential $v_{\text {eff }, \lambda}$ is added that meanwhile follows a path in the space of potentials in order to keep the density constant. The energy difference for the initial and final states of the path is given by eq. (3.118) and is added to the energy of the system of interacting electrons to keep the energy constant. This justifies the transition in eq. (3.110).

As we will see in section 3.9.5, the expression of the exchange-correlation energy in terms of the integrated hole function, eq. (3.118), is utilized in the development of hybrid functionals.

\subsubsection{Spin-polarized systems}

So far we discussed the basics of density functional theory for systems without a spin-dependence. However, often the ground state of a material is spinpolarized. This inevitably occurs in systems with an odd electron number or, e. g., for magnetic materials and happens even if the external potential is spin-independent. Furthermore, a spin-dependent theory has the advantage that external magnetic fields can be applied in material simulations. In this case the external magnetic field can be merged into the external potential which consequently receives a dependence on the spin.

The Levy and Lieb functionals can be extended to a spin-polarized formulation in terms of the electron densities of both spin directions, $n_{\uparrow}(\vec{r})$ and $n_{\downarrow}(\vec{r})$, with $n(\vec{r})=n_{\uparrow}(\vec{r})+n_{\downarrow}(\vec{r})[68,69]$. Levy's constrained search can be performed over all wave functions $|\Psi\rangle$ that at the same time produce the densities $n_{\uparrow}(\vec{r})$ and $n_{\downarrow}(\vec{r})$,

$$
F_{\text {Levy }}\left[n_{\uparrow}, n_{\downarrow}\right]=\min _{|\Psi\rangle: n_{\uparrow}=\left\langle\Psi\left|\hat{n}_{\uparrow}\right| \Psi\right\rangle, n_{\downarrow}=\left\langle\Psi\left|\hat{n}_{\downarrow}\right| \Psi\right\rangle}\langle\Psi|(\hat{T}+\hat{U})| \Psi\rangle .
$$


The extended version of Lieb's ensemble state density functional reads

$$
F_{\text {Lieb }}\left[n_{\uparrow}, n_{\downarrow}\right]=\min _{\{w\},\{|\Psi\rangle\}:\left\langle\Psi_{i}\left|\hat{n}_{\sigma}\right| \Psi_{i}\right\rangle=n_{i, \sigma}, \sum_{i} w_{i} n_{i, \sigma}=n_{\sigma}} \sum_{i} w_{i}\left\langle\Psi_{i}|(\hat{T}+\hat{U})| \Psi_{i}\right\rangle,
$$

where $\sigma \in\{\uparrow, \downarrow\}$ and the set of ensemble weights obeys the conditions $w_{i} \geq$ $0, \sum_{i} w_{i}=1$.

\subsubsection{The Kohn-Sham equations}

In the following, we show the spin-polarized and extended version of the original Kohn-Sham approach to DFT [68, 69, 71-73]. This computational scheme may be used to calculate the ground state energy and the ground state spin densities. For the remainder of this section, we employ the Lieb functional $F\left[n_{\uparrow}, n_{\downarrow}\right] \equiv F_{\text {Lieb }}\left[n_{\uparrow}, n_{\downarrow}\right]$ in the extended formulation for spin-polarized systems. The decomposition of Lieb's universal density functional for the spin-resolved case is analogous to eq. (3.110) and reads

$$
F\left[n_{\uparrow}, n_{\downarrow}\right]=T_{s}\left[n_{\uparrow}, n_{\downarrow}\right]+E_{\mathrm{H}}[n]+E_{\mathrm{xc}}\left[n_{\uparrow}, n_{\downarrow}\right] .
$$

The electron density is given as the sum of the two spin densities, $n(\vec{r})=$ $n_{\uparrow}(\vec{r})+n_{\downarrow}(\vec{r})$. We can write the non-interacting kinetic energy functional in terms of a set of non-interacting wave functions $\psi_{n}(\vec{r}, \sigma)$, called the Kohn-Sham wave functions, and their respective occupancy numbers $0 \leq f_{n} \leq 1$,

$$
T_{S}\left[n_{\uparrow}, n_{\downarrow}\right]=\min _{\{\psi\},\{f\}} \sum_{n} f_{n} \int \mathrm{d} \vec{r} \sum_{\sigma} \psi_{n}^{*}(\vec{r}, \sigma)\left(-\frac{\hbar^{2}}{2 m} \vec{\nabla}^{2}\right) \psi_{n}(\vec{r}, \sigma) .
$$

The spin densities are obtained via

$$
n_{\sigma}(\vec{r})=\sum_{n} f_{n}\left|\psi_{n}(\vec{r}, \sigma)\right|^{2}
$$

Our notation includes an index $n$, labeling the band and the spin of the KohnSham states. Moreover, the wave function coordinates are the real space vector and the spin. If the spin component of $n$ and the spin $\sigma$ do not coincide, the wave function vanishes.

The minimization in eq. (3.122) is performed subject to two constraints. First, the wave functions $\psi_{n}(\vec{r}, \sigma)$ shall obey the orthonormality condition

$$
\sum_{\sigma} \int \mathrm{d} \vec{r} \psi_{n}^{*}(\vec{r}, \sigma) \psi_{m}(\vec{r}, \sigma)=\delta_{n m}
$$

Second, the occupancies $f_{n}$ sum to the number of particles in the system,

$$
\sum_{n} f_{n}=N
$$

To derive the extended Kohn-Sham scheme, we write down a functional for 
the total energy, for which the functional variables are the wave functions $\{\psi\}$ and the respective occupancies $\{f\}$,

$$
\begin{aligned}
E[\{\psi\},\{f\}]=\sum_{n} & f_{n} \int \mathrm{d} \vec{r} \sum_{\sigma} \psi_{n}^{*}(\vec{r}, \sigma)\left(-\frac{\hbar^{2}}{2 m} \vec{\nabla}^{2}\right) \psi_{n}(\vec{r}, \sigma) \\
& +E_{\mathrm{H}}[n]+E_{\mathrm{xc}}\left[n_{\uparrow}, n_{\downarrow}\right]+\int \mathrm{d} \vec{r} \sum_{\sigma} v_{\mathrm{ext}}^{\sigma}(\vec{r}) n_{\sigma}(\vec{r}) .
\end{aligned}
$$

The spin densities are given by eq. (3.123). We include the orthonormality constraint (3.124) and the particle number constraint (3.125) via Lagrange multipliers $\boldsymbol{\Lambda}$ and $\mu$, which leads to the functional

$$
\begin{gathered}
\tilde{E}[\{\psi\},\{f\}]=E[\{\psi\},\{f\}]-\sum_{n m} \Lambda_{n m}\left[\sum_{\sigma} \int \mathrm{d} \vec{r} \psi_{n}^{*}(\vec{r}, \sigma) \psi_{m}(\vec{r}, \sigma)-\delta_{n m}\right] \\
-\mu\left[\sum_{n} f_{n}-N\right]
\end{gathered}
$$

Note that, since the energy should stay real, $\boldsymbol{\Lambda}$ needs to be a Hermitian matrix.

We independently minimize the functional (3.127) with respect to the wave functions $\{\psi\}$ and the occupancies $\{f\}$. First, for the minimization with respect to the wave functions, the Wirtinger derivative of eq. (3.127) with respect to $\psi_{n}^{*}$ and the minimization condition $\delta \tilde{E} / \delta \psi_{n}^{*} \stackrel{!}{=} 0$ lead to the Schrödinger-like equation

$$
f_{n}\left(-\frac{\hbar^{2}}{2 m} \nabla^{2}\right) \psi_{n}(\vec{r}, \sigma)+f_{n} v_{\mathrm{eff}}^{\sigma}(\vec{r}) \psi_{n}(\vec{r}, \sigma)=\sum_{n m} \Lambda_{n m} \psi_{m}(\vec{r}, \sigma),
$$

where the effective potential is defined as

$$
v_{\mathrm{eff}}^{\sigma}\left[n_{\uparrow}, n_{\downarrow}\right]=v_{\mathrm{ext}}^{\sigma}\left[n_{\uparrow}, n_{\downarrow}\right]+v_{\mathrm{H}}[n]+v_{\mathrm{xc}}^{\sigma}\left[n_{\uparrow}, n_{\downarrow}\right] .
$$

In eq. (3.129) $v_{\mathrm{H}}[n]=\delta E_{\mathrm{H}}[n] / \delta n$ is the Hartree potential and $v_{\mathrm{xc}}^{\sigma}\left[n_{\uparrow}, n_{\downarrow}\right]=$ $\delta E_{\mathrm{xc}} / \delta n_{\sigma}$ denotes the exchange-correlation potential. The left hand side of eq. (3.128) has the form $f_{n} \hat{h}_{\sigma}(\vec{r}) \psi_{n}(\vec{r}, \sigma)$, where

$$
\hat{h}_{\sigma}(\vec{r})=\left(-\frac{\hbar^{2}}{2 m} \nabla^{2}\right)+v_{\mathrm{eff}}^{\sigma}(\vec{r})
$$

Equation (3.130) is a Hermitian operator that we use to set up the Schrödinger equation

$$
\hat{h}_{\sigma}(\vec{r}) \psi_{n}(\vec{r}, \sigma)=\left[\left(-\frac{\hbar^{2}}{2 m} \nabla^{2}\right)+v_{\mathrm{eff}}^{\sigma}(\vec{r})\right] \psi_{n}(\vec{r}, \sigma)=\varepsilon_{n} \psi_{n}(\vec{r}, \sigma),
$$

which defines the eigenvalues $\varepsilon_{n}$ and is known as Kohn-Sham equation. Using eq. (3.131), a solution for eq. (3.128) is $\Lambda_{n m}=f_{n} \varepsilon_{n} \delta_{n m}$.

Note that, in the minimum of the energy functional (3.126) with respect to 
the wave functions, the equations (3.129) and (3.131) can be exploited to show that the derivative of the total energy functional (3.126) with respect to the occupancy $f_{n}$ reads

$$
\frac{\partial E}{\partial f_{n}}=\varepsilon_{n}
$$

Equation (3.132) is known as Janak's theorem [74].

Next, we minimize the functional (3.127) with respect to the occupancies $\{f\}$. Since we have $0 \leq f_{n} \leq 1$, we define

$$
f_{n}=\cos ^{2}\left(\vartheta_{n}\right)
$$

for each $n$ and, instead of varying over the set $\{f\}$, we vary over the set $\{\vartheta\}$. The minimization condition $\partial \tilde{E} / \partial \vartheta_{n} \stackrel{!}{=} 0$ yields

$$
\frac{\partial \tilde{E}}{\partial \vartheta_{n}}=\frac{\partial \tilde{E}}{\partial f_{n}} \frac{\mathrm{d} f_{n}}{\mathrm{~d} \vartheta_{n}}=\left[\varepsilon_{n}-\mu\right] \sin \left(2 \vartheta_{n}\right)=0 .
$$

From eq. (3.134) it is clear that the total energy functional (3.126) is minimized for a fixed set of Kohn-Sham wave functions if the occupancy of each KohnSham state is one of the following. If $\varepsilon_{n}=\mu$ the choice of the number $\vartheta_{n}$ is arbitrary and the occupancy is an arbitrary value $0 \leq f_{n} \leq 1$. For the case $\varepsilon_{n} \neq \mu$ we have either $\vartheta_{n}=0$ or $\vartheta_{n}=\pi / 2$, which correspond to $f_{n}=1$ and $f_{n}=0$, respectively. The minimum of the total energy is usually obtained by filling the Kohn-Sham states with the smallest energies from the bottom [68]. Only those Kohn-Sham states that lie at the Fermi level $E_{\mathrm{F}}$ can have a fractional occupancy and we have $\mu=E_{\mathrm{F}}$.

In practical DFT calculations the aim is to find the Kohn-Sham system that minimizes the total energy (3.126) and produces the ground state density. This is often done by solving eqs. (3.123), (3.129) and (3.131), and occupying the lowest Kohn-Sham wave functions in an iterative scheme until self-consistency is reached. We use an alternative approach based on a functional minimization procedure which is described in section 3.10.

Although the Kohn-Sham wave functions are auxiliary wave functions with no physical meaning, they and their corresponding one-particle energies are in practice taken as an approximation to real one-particle states and related energies of the simulated system. This allows the calculation of the band structure and the density of states.

Note that the exact form of the exchange correlation functional $E_{\mathrm{xc}}\left[n_{\uparrow}, n_{\downarrow}\right]$ remains unknown and it has to be approximated for practical calculations. In the upcoming section we summarize some of the most important approximations to $E_{\mathrm{xc}}\left[n_{\uparrow}, n_{\downarrow}\right]$ and present the exchange correlation functional that is mainly used in the context of this thesis.

\subsection{The exchange-correlation functional}

In the Kohn-Sham formulation of density functional theory, the exchangecorrelation functional is the sole unknown part of the total energy functional 
and hence has to be approximated. Since the success of DFT simulations relies heavily on the performance of the exchange-correlation functional, various approximate forms have been formulated since the advent of DFT. In the following sections, a brief overview of the development of density functional approximation for the exchange-correlation energy is given, which leads to today's most accurate forms of the exchange-correlation functional.

\subsubsection{The local density approximation}

A simple, nevertheless very important approximation for the exchange-correlation functional is the local density approximation (LDA) (see e.g. [68]). The LDA utilizes the exchange-correlation energy per particle $\varepsilon_{\mathrm{xc}}^{\mathrm{HEG}}(n)$ of the homogeneous electron gas (HEG), where $n$ is a constant density for the HEG. With the assumption that the electron density of DFT is slowly varying in space, the exchange correlation energy functional can be approximated by [67]

$$
E_{\mathrm{xc}}^{\mathrm{LDA}}[n]=\int \mathrm{d} \vec{r} n(\vec{r}) \varepsilon_{\mathrm{xc}}^{\mathrm{HEG}}(n(\vec{r}))
$$

In eq. (3.135) the constant density of the homogeneous electron gas is replaced by the local density of DFT, $n \rightarrow n(\vec{r})$, hence the name "local density approximation".

\subsubsection{The local spin density approximation}

A generalization of the LDA to spin-polarized DFT is the local spin density approximation (LSDA), see e.g. [55, 68]. In this case the exchange correlation functional depends on the partial densities of the spin directions $n_{\uparrow}$, and $n_{\downarrow}$,

$$
E_{\mathrm{xc}}^{\mathrm{LSDA}}\left[n_{\uparrow}, n_{\downarrow}\right]=\int \mathrm{d} \vec{r} n(\vec{r}) \varepsilon_{\mathrm{xc}}^{\mathrm{HEG}}\left(n_{\uparrow}(\vec{r}), n_{\downarrow}(\vec{r})\right) .
$$

or, alternatively, on the total density $n$ and the spin polarization $\zeta=\left(n_{\uparrow}-\right.$ $\left.n_{\downarrow}\right) /\left(n_{\uparrow}+n_{\downarrow}\right)$.

$\varepsilon_{\mathrm{xc}}^{\mathrm{HEG}}$ can be split into an exchange part $\varepsilon_{\mathrm{x}}^{\mathrm{HEG}}$ and a correlation part $\varepsilon_{\mathrm{c}}^{\mathrm{HEG}}$. The exchange contribution $\varepsilon_{\mathrm{x}}^{\mathrm{HEG}}$ is known analytically [75], while the correlation part is modeled via a parametrized function, for which various forms have been suggested. Three often considered parametrizations, which interpolate the results of quantum Monte Carlo calculations for the correlation energy per particle of Ceperley and Alder [76], are given by Vosko, Wilk, and Nusair [77], Perdew and Zunger [71], and Perdew and Wang [78, 79].

Because the LSDA is based on the assumption of a spatially slowly varying electron density, one might expect the LSDA to be a good approximation for rather homogeneous systems only. However, the LSDA turns out to often give reasonably accurate results also in the case of systems with rather inhomogeneous electron densities. Two common explanations for this observation [68, $69,80,81]$ are outlined in the following. 
First, it can be shown that, for an interaction $w\left(\vec{r}, \vec{r}^{\prime}\right)=w\left(\left|\vec{r}-\vec{r}^{\prime}\right|\right)$, the spherical average of the exchange-correlation hole (eq. (3.117)) enters the exchange correlation energy functional (3.118). Hence, the LSDA does not need to produce a good approximation for the exchange correlation hole, but rather only for its spherical average. Indeed, to name two examples, it was shown by Gunnarsson, Jonson, and Lundqvist for a neon atom [81] and by Jones and Gunnarsson for a nitrogen atom [82] that the spherical average of the exchange hole in the LSDA is reasonably close to the spherical average of the exact exchange hole, while the exchange hole itself is not well represented in the LSDA.

Second, the exchange correlation hole of the LDA satisfies the sum rule eq. (3.6) so that errors in the exchange correlation hole of the LSDA, which may be positive for one location $\vec{r}$ and negative for a different location $\vec{r}^{\prime}$, have to cancel to a certain degree.

While the LSDA turned out to be successful in many applications even beyond the regime of a spatially slowly varying density, it has some deficiencies, see e. g. $[68,83]$. For example, the LSDA is known to overestimate the binding energies in molecules [84], which is commonly referred to as "overbinding". This problem renders the results with the LSDA unsatisfactory in many applications in chemistry. Some of the shortcomings of the LSDA are resolved by the generalized gradient approximation, which is introduced in the next section.

\subsubsection{The generalized gradient approximation}

It is clear that the assumption of a spatially slowly varying density, as in the LSDA, does not reflect the situation in a material in which spatial variations of the density are rather large. One possibility to account for inhomogeneities of the density is to include a dependence on the density gradient in the energy $\varepsilon_{\mathrm{xc}}$. This is done in the so-called generalized gradient approximation (GGA). In the GGA, the exchange correlation energy per particle is replaced according to

$$
\varepsilon_{\mathrm{xc}}^{\mathrm{HEG}}\left(n_{\uparrow}(\vec{r}), n_{\downarrow}(\vec{r})\right) \rightarrow \varepsilon_{\mathrm{xc}}\left(n_{\uparrow}(\vec{r}), n_{\downarrow}(\vec{r}), \nabla n_{\uparrow}(\vec{r}), \nabla n_{\downarrow}(\vec{r})\right) .
$$

The new energy quantities can be represented as the energies of the homogeneous electron gas, corrected by gradient-dependent functions. A GGA exchange-correlation functional can be expressed as $[55,83]$

$$
\begin{aligned}
E_{\mathrm{xc}}^{\mathrm{GGA}}\left[n_{\uparrow}, n_{\downarrow}\right]= & \int \mathrm{d} \vec{r} n \varepsilon_{\mathrm{xc}}\left(n_{\uparrow}, n_{\downarrow}, \nabla n_{\uparrow}, \nabla n_{\downarrow}\right) \\
= & \int \mathrm{d} \vec{r} n\left[\varepsilon_{\mathrm{x}}^{\mathrm{HEG}}\left(n_{\uparrow}, n_{\downarrow}\right) F_{\mathrm{x}}\left(n_{\uparrow}, n_{\downarrow}, \nabla n_{\uparrow}, \nabla n_{\downarrow}\right)\right. \\
& \left.+\varepsilon_{\mathrm{c}}^{\mathrm{HEG}}\left(n_{\uparrow}, n_{\downarrow}\right) F_{\mathrm{c}}\left(n_{\uparrow}, n_{\downarrow}, \nabla n_{\uparrow}, \nabla n_{\downarrow}\right)\right] .
\end{aligned}
$$

There exist several different approximations for $F_{\mathrm{x}}$ and $F_{\mathrm{c}}$, each trying to recover certain physical properties in the region of large density gradients. The 
most widely known GGA functionals are the B88-functional by Becke [85], the PW91-functional by Perdew and Wang $[78,79]$, and the most widely used PBE-functional by Perdew, Burke, and Ernzerhof [86].

GGA functionals improve on some of the shortcomings of the LSDA, like the overbinding of chemical bonds, and often a GGA functional is used in DFT simulations.

\subsubsection{Extensions to the LSDA and GGA}

Many materials are surprisingly well described when using LSDA or GGA functionals. However, for materials with strong electron-electron interactions results of LSDA or GGA calculations are often unsatisfactory. Among the prominent examples of materials, for which the LSDA breaks down, are the transition-metal monoxides. For $\mathrm{NiO}$ and $\mathrm{MnO}$, the ground state magnetic structure is described correctly, however, the band gap is hugely underestimated [87-89]. Moreover, $\mathrm{FeO}$ and $\mathrm{CoO}$ are even found to be metals, although they are insulators [88].

The band gap problem was traced back to a discontinuity in the functional derivative of the total energy w.r.t. the density, $\delta E / \delta n$, that is present in an exact energy functional of the density, but missing in the LSDA [89-93]. In an open system, given by an atom that exchanges particles with a reservoir, the dependence of the ground state total energy $E$ on the number of particles $N$ is a collection of straight lines that are joined in value at integer particle numbers. When moving across an integer particle number in the total energy graph, a kink is crossed which leads to a discontinuity in $\partial E / \partial N$ and also in $\delta E / \delta n$. The size of a bandgap is directly related to the size of the derivative discontinuity. Because in the LSDA the derivative $\delta E_{\mathrm{xc}}^{\mathrm{LSDA}} / \delta n$ is continuous, no derivative discontinuity is present and the band gap is underestimated.

For the case of strongly correlated materials, a source of a potentially large error is the self-interaction of an electron. In DFT, the interaction energy is split into the Hartree part and the exchange-correlation contribution. Since the Hartree part incorporates the interaction of an electron with itself, this self-interaction has to be subtracted in the exchange-correlation contribution. However, LSDA and GGA exchange-correlation functionals do not compensate the self-interaction error exactly. Hence, in the case of a large Coulomb interaction, the self-interaction error can become severe. The self-interaction increases the energy of the occupied states so that the energy separation of occupied and unoccupied states is reduced.

To resolve the problems of DFT with LSDA or GGA functionals, several methods have been developed. Among these are the so-called hybrid functionals, which are applied in the simulations in connection with this thesis. Motivated by eqs. (3.117) and (3.118), originating from the adiabatic connection, hybrid functionals mix a portion of exact Hartree-Fock exchange to the LSDA or GGA density functional approximation. Because in Hartree-Fock the exchange term cancels the self-interaction of the Hartree term, hybrid functionals improve upon the insufficient treatment of the self-interaction in the LSDA and GGA. An overview of the hybrid functional theory is given in the next 
section.

Other approaches include the self-interaction correction method (SIC) [94], in which the LSDA is used in conjunction with an additional term for the correction of the self-interaction error of the LSDA. Moreover, the LDA+ $U$ method $[89,95,96]$ uses the LDA in conjunction with an additional orbitaldependent treatment of localized states. Extended non-interacting states of the system are handled in the LDA, while for localized states the interaction energy is evaluated in Hartree-Fock approximation in a local orbital basis. A doublecounting correction is needed to subtract those contributions for the localized orbitals that are accounted for by both the LDA and the orbital-dependent interaction term. The LDA $+U$ methods reintroduces a discontinuity in the potential, which is absent in the LSDA but important for the description of band gaps.

Moreover, dynamical mean-field theory (DMFT) $[97,98]$ is used in conjunction with DFT [99-101]. In many-particle theory, DMFT is used to perform a mapping of a many-body lattice model to a single site impurity, embedded in an effective bath. The mapping between the lattice system and the impurity is determined in a self-consistency cycle. It is exact in infinite dimensions and used for an approximation of the three-dimensional case. In a computational scheme involving DFT and DMFT, a converged DFT simulation can be used as an input for the DMFT, from which a corrected spectral function can be obtained. Both methods can also be intertwined in a self-consistency cycle, in which case a correction for the density from the DMFT is fed back into the DFT. A combination of DFT and DMFT has the advantage that the local orbitals are treated in a many-body fashion and the solution of the local problem is not restricted to a single Slater determinant. However, a double counting correction is needed that accounts for a correction of both, the exchange and the correlation part. Furthermore, solving the impurity model is computationally expensive. Even with modern impurity solvers a treatment of a full $d$ or, especially, a full $f$ shell is challenging.

\subsubsection{Hybrid functionals}

In search of an improvement on GGA functionals, eventually hybrid functionals have been introduced. The name hybrid functional stems from the binary form the exchange-correlation energy functional takes in this approach - a combination of the exactly known Hartree-Fock exchange as one constituent and a density functional approximation, like the GGA, as the other contribution.

The general idea behind the hybrid functionals was presented by Becke [102] and is based on the adiabatic connection, discussed in section 3.8.4. Becke argues that the modeling of the exchange-correlation functional via the homogeneous electron gas is inappropriate for the lower bound of eq. (3.117), where $\lambda=0$. For $\lambda=0$, the system consists of independent particles, so that correlations do not exist and $h_{0}\left(\vec{r}, \vec{r}^{\prime}\right)$ is the exchange hole known from HartreeFock theory. Using a local density functional approximation like LSDA or GGA introduces correlations, even in the uncorrelated regime and leads to inaccurate results. 
To alleviate this problem, in the integration eq. (3.117) the properties of the hole function for the complete range of interaction strengths $\lambda$ have to be accounted for more carefully. The first and simplest approach proposed by Becke [102] is to interpolate the integral (3.117) linearly, so that the integrated exchange-correlation hole becomes

$$
\bar{h}_{\mathrm{xc}}\left(\vec{r}, \vec{r}^{\prime}\right)=\frac{1}{2} h_{\lambda=0}\left(\vec{r}, \vec{r}^{\prime}\right)+\frac{1}{2} h_{\lambda=1}\left(\vec{r}, \vec{r}^{\prime}\right)
$$

According to Hartree-Fock theory, the exchange-correlation potential associated with $h_{\lambda=0}$ is the Hartree-Fock exchange energy, $U_{\mathrm{xc}, 0}=E_{\mathrm{x}}^{\mathrm{HF}}$, which is known analytically. For the case $\lambda=1$, Becke proposed to use the exchangecorrelation potential of LSDA, $U_{\mathrm{xc}}^{\mathrm{LSDA}}$, so that

$$
E_{\mathrm{xc}} \approx \frac{1}{2} E_{\mathrm{x}}^{\mathrm{HF}}+\frac{1}{2} U_{\mathrm{xc}}^{\mathrm{LSDA}}
$$

Early refinements of the hybrid functional approach are the B3PW91 functional [103] with the general form

$E_{\mathrm{xc}}=E_{\mathrm{xc}}^{\mathrm{LSDA}}+a_{0}\left(E_{\mathrm{x}}^{\mathrm{HF}}-E_{\mathrm{x}}^{\mathrm{LSDA}}\right)+a_{\mathrm{x}}\left(E_{\mathrm{x}}^{\mathrm{GGA}}-E_{\mathrm{x}}^{\mathrm{LSDA}}\right)+a_{\mathrm{c}}\left(E_{\mathrm{c}}^{\mathrm{GGA}}-E_{\mathrm{c}}^{\mathrm{LSDA}}\right)$

and the similarly shaped B3LYP functional [104]. Both functionals use the Becke88 (B88) gradient correction for exchange and depend on three parameters, $a_{0}, a_{\mathrm{x}}$, and $a_{\mathrm{c}}$ that have to be determined through fitting empirical data. Moreover, the B3PW91 functional uses the Perdew-Wang91 (PW91) correlation-correction, whereas the B3LYP functional employs a combination of the Lee-Yang-Parr (LYP) [105] and the Vosko-Wilk-Nusair (VWN) [77] correlation corrections.

In further work, the number of fit parameters was reduced to just one by employing a new correlation functional [106]. The exchange-correlation energy functional then is of the form

$$
E_{\mathrm{xc}}=E_{\mathrm{xc}}^{\mathrm{DFA}}+\eta\left(E_{\mathrm{x}}^{\mathrm{HF}}-E_{\mathrm{x}}^{\mathrm{DFA}}\right) .
$$

Fits to experimental data showed best matches for $\eta=0.16$ and $\eta=0.28$, depending on the $E_{\mathrm{xc}}^{\mathrm{DFA}}$ functional [106]. Furthermore, a mixing factor $\eta=1 / 4$ was suggested, based on perturbation theory calculations [107].

The parameters of the hybrid functionals presented so far were determined by fitting to experimental data, often from a database for molecular data. Hence, the hybrid functionals relying on these specific parameter sets are assumed to be biased towards use cases similar to that of the fitted data and less accurate for other systems. Due to this reason, a hybrid functional of the form eq. (3.143), based on the PBE functional, with $\eta=1 / 4$ was introduced: the zero-adjustableparameter PBEO functional. The PBE0 functional was found to often reach a similar level of accuracy as the fitted functionals $[108,109]$. Hybrid functionals like the PBE0 functional that treat the exchange mixing uniformly in space are often categorized as global hybrid functionals. While global hybrid functionals 
improve upon some of the shortcomings of GGA functionals, e.g. the treatment of the self-interaction, the uniform treatment of the exchange contribution leads to other problems. One major drawback is the rather high computational effort needed to perform simulations with the PBE0 functional, which is related to the long-range nature of the exact exchange [110,111].

The deficiencies of the PBE0 hybrid functional motivated the development of range-separated hybrid functionals, in which the short-range and long-range parts of the exchange are treated individually. For solid state simulations a well-known example is the HSE functional $[112,113]$. In the HSE functional only the short-range contribution is treated in the Hartree-Fock manner, while the long-range part is treated on the DFT level,

$$
E_{\mathrm{xc}}^{\mathrm{HSE}}=E_{\mathrm{x}}^{\mathrm{PBE}, \mathrm{SR}}+\eta\left(E_{\mathrm{x}}^{\mathrm{HF}, \mathrm{SR}}-E_{\mathrm{x}}^{\mathrm{PBE}, \mathrm{SR}}\right)+E_{\mathrm{x}}^{\mathrm{PBE}, \mathrm{LR}}+E_{\mathrm{c}}^{\mathrm{PBE}} .
$$

A constant factor defines the distance of the short-range to long-range transition. The range-separated treatment of the Coulomb interaction renders the HSE functional computationally less expensive than the PBE0 functional $[110,111]$.

A further class of functionals, designed to remedy the shortcomings of global hybrid functionals, are so-called local hybrid functionals (see e. g. [114, 115]) in which the exchange admixture is position-dependent. For simulations in the context of this thesis we use the local hybrid functional PBE0r, which is discussed in section 3.9.6.

\subsubsection{The PBEOr hybrid functional}

DFT hybrid functional simulations in the context of this thesis are performed with the PBE0r hybrid functional [116]. The PBE0r hybrid functional is a local hybrid functional in which the Hartree-Fock exchange admixture is computed as a sum of on-site exchange contributions that are obtained in a local orbital basis. For each site in the unit cell an individual exchange mixing factor is specified. By restricting the exact exchange contributions to the on-site terms the treatment of the long-range inter-site exchange contributions remains on the level of the density functional approximation. Rigorously stated, the PBE0r functional is of the form

$$
E_{\mathrm{xc}}^{\mathrm{PBE} 0 \mathrm{r}}=E_{\mathrm{xc}}^{\mathrm{PBE}}+\sum_{A} \eta_{A}\left(E_{\mathrm{x}, A}^{\mathrm{HF}}-E_{\mathrm{x}, A}^{\mathrm{DC}}\right)
$$

where $E_{\mathrm{xc}}^{\mathrm{PBE}}$ is the $\mathrm{PBE}$ functional and $E_{\mathrm{x}, A}^{\mathrm{HF}}$ is the on-site Hartree-Fock exchange energy of site $A . E_{\mathrm{x}, A}^{\mathrm{DC}}$ is a double counting correction, which subtracts exchange contributions that are accounted for by both $E_{\mathrm{xc}}^{\mathrm{PBE}}$ and $E_{\mathrm{x}, A}^{\mathrm{HF}}$. The mixing strength is controlled for each site $A$ by the factor $\eta_{A}$.

For determining the exchange energy contribution $E_{\mathrm{x}, A}^{\mathrm{HF}}$ and the double counting correction $E_{\mathrm{x}, A}^{\mathrm{DC}}$ the Kohn-Sham wave functions are projected onto a set of localized tight-binding orbitals that are determined for each atomic species in the simulation cell. I. e., for each atomic site $A$ a set of local orbitals $\left\{\left|\chi_{A}\right\rangle\right\}$ is constructed, where for each local orbital $\left|\chi_{A a}\right\rangle$ there exists a corresponding 
local orbital projector $\left|\pi_{A a}\right\rangle$. The local basis projectors are constructed such that the deviation of a state

$$
\left|\psi^{\prime}\right\rangle=\sum_{A} \sum_{a \in A}\left|\chi_{A a}\right\rangle\left\langle\pi_{A a} \mid \psi\right\rangle
$$

from the Kohn-Sham state $|\psi\rangle$ is minimal.

For a site $A$ of tight-binding orbitals the one-particle density matrix is obtained via a projection of the Kohn-Sham occupancies $f_{n}$ onto the tight-binding orbitals,

$$
\rho_{A, a b}=\sum_{n}\left\langle\pi_{A a} \mid \psi_{n}\right\rangle f_{n}\left\langle\psi_{n} \mid \pi_{A b}\right\rangle
$$

In the basis of the on-site tight-binding orbitals the exchange energy $E_{\mathrm{x}, A}^{\mathrm{HF}}$ of site $A$ is computed via

$$
E_{\mathrm{x}, A}^{\mathrm{HF}}=-\frac{1}{2} \sum_{a b c d \in A} \rho_{A, c d} U_{A, d a b c} \rho_{A, b a},
$$

where the Coulomb tensor elements for each site are computed by using the Laplace expansion of the Coulomb interaction (see e. g. [117]).

For later reference, let us state important derivatives of the exchange energy (3.148). We have

$$
\begin{aligned}
\mathrm{d} E_{\mathrm{x}, A}^{\mathrm{HF}}= & \sum_{a b \in A} \frac{\partial E_{\mathrm{x}, A}^{\mathrm{HF}}}{\partial \rho_{A, b a}} \mathrm{~d} \rho_{A, b a} \\
= & \sum_{a b \in A} \frac{\partial E_{\mathrm{x}, A}^{\mathrm{HF}}}{\partial \rho_{A, b a}} \sum_{n}\left[\left\langle\pi_{A b} \mid \psi_{n}\right\rangle\left\langle\psi_{n} \mid \pi_{A a}\right\rangle \mathrm{d} f_{n}\right. \\
& \left.\quad+f_{n}\left\langle\psi_{n} \mid \pi_{A a}\right\rangle\left\langle\pi_{A b}|\mathrm{~d}| \psi_{n}\right\rangle+f_{n}\left\langle\pi_{A b} \mid \psi_{n}\right\rangle\left(\mathrm{d}\left\langle\psi_{n}\right|\right)\left|\pi_{A a}\right\rangle\right],
\end{aligned}
$$

where we inserted eq. (3.147) in the second step. Moreover, the derivative of eq. (3.148) with respect to the density matrix is

$$
\frac{\partial E_{\mathrm{x}, A}^{\mathrm{HF}}}{\partial \rho_{A, b a}}=-\sum_{c d \in A} \rho_{A, c d} U_{A, d a b c} .
$$

Let us define the single-particle operator

$$
\hat{h}_{\mathrm{x}, A}^{\chi}:=\sum_{a b \in A}\left|\pi_{A a}\right\rangle \frac{\partial E_{\mathrm{x}, A}^{\mathrm{HF}}}{\partial \rho_{A, b a}}\left\langle\pi_{A b}\right| .
$$

From eq. (3.150) we obtain the derivatives

$$
\frac{\partial E_{\mathrm{x}, A}^{\mathrm{HF}}}{\partial f_{n}}=\left\langle\psi_{n}\left|\hat{h}_{\mathrm{x}, A}^{\chi}\right| \psi_{n}\right\rangle
$$


and

$$
\frac{\partial E_{\mathrm{x}, A}^{\mathrm{HF}}}{\partial\left\langle\psi_{n}\right|}=f_{n} \hat{h}_{\mathrm{x}, A}^{\chi}\left|\psi_{n}\right\rangle .
$$

According to Janak's theorem, eq. (3.132), equation (3.153) represents the contribution of the exact exchange of a site $A$ to the energy of a Kohn-Sham state. Equation (3.154) is a contribution of the exact exchange of a site $A$ that enters the Schrödinger-like equation (3.128).

Let us now come to the double-counting correction. From the sets of local orbital states, the two densities

$$
n_{A}^{\chi}(\vec{r})=\sum_{a, b \in A} \sum_{n \sigma} f_{n}\left\langle\psi_{n} \mid \pi_{A a}\right\rangle\left\langle\chi_{A a} \mid \vec{r}, \sigma\right\rangle\left\langle\vec{r}, \sigma \mid \chi_{A b}\right\rangle\left\langle\pi_{A b} \mid \psi_{n}\right\rangle
$$

and

$$
n^{\chi}(\vec{r})=\sum_{A} n_{A}^{\chi}(\vec{r})
$$

can be defined, where eq. (3.155) is the electron density coming from the local orbitals of site $A$ and eq. (3.156) is the electron density coming from all local orbitals in the unit cell. With the densities (3.155) and (3.156) of the on-site tight-binding orbitals, the double counting correction $E_{\mathrm{x}, A}^{\mathrm{DC}}$ reads (cf. [118])

$$
E_{\mathrm{x}, A}^{\mathrm{DC}}=\int \mathrm{d} \vec{r} n_{A}^{\chi}(\vec{r}) \varepsilon_{\mathrm{xc}}\left[n_{\sigma, \sigma^{\prime}}^{\chi}(\vec{r})\right] \frac{n_{A}^{\chi}(\vec{r})}{n^{\chi}(\vec{r})},
$$

where $\varepsilon_{\mathrm{xc}}$ is the exchange-correlation energy per electron as in the PBE functional.

The approach of using only on-site exchange is well suited for materials with localized $d$ or $f$ orbitals, such as transition metals or rare-earths. However, it is inappropriate in systems with strong covalent bonds [34].

\subsection{Car-Parrinello dynamics}

Until now it was mainly discussed, how to find the ground state electronic structure of an electronic many-particle system in an external potential, which is given by fixed ion positions. However, to find the ground state of a system of ions and electrons, the energy has to be minimized with respect to the electronic, as well as the ionic degrees of freedom and both are obviously coupled. One way to find a solution is to guess an initial set of ion positions and solve the electronic structure problem via the Kohn-Sham equations. Based on the result of the electronic structure calculation, the ion positions are updated and the electronic structure is computed again for the new set of ion positions. This cycle can be repeated until convergence. A major drawback of this so-called Born-Oppenheimer molecular dynamics is that the computationally expensive wave function optimization has to be performed in each step.

In 1985 R. Car and M. Parrinello introduced a different and computationally less demanding approach for ab-initio molecular dynamics [57]. Within the 
new scheme the electronic states and ion positions are optimized simultaneously rather than separately, as in the Born-Oppenheimer molecular dynamics. The key idea is to treat both, the ion positions and the electronic wave functions, as classical variables that evolve in time towards their optimum values according to their respective classical equations of motion. However, it has to be emphasized that the equation of motion for the wave function does not describe the realworld time evolution of the quantum mechanical Kohn-Sham states, but should rather be regarded as a technical instruction to optimize the Kohn-Sham states.

The optimization scheme employed in the CP-PAW simulation code, which is used in the context of this thesis, is based on the Car-Parrinello concept. At the heart of the method is the Lagrangian

$$
\begin{gathered}
L=\sum_{n} f_{n}\left\langle\dot{\psi}_{n}\left|m_{\psi}\right| \dot{\psi}_{n}\right\rangle+\frac{1}{2} \sum_{i} m_{\vec{R}} \dot{\vec{R}}_{i}^{2}-E[\{\psi\},\{\vec{R}\}] \\
-\sum_{m n} \Lambda_{m n}\left[\left\langle\psi_{m} \mid \psi_{n}\right\rangle-\delta_{m n}\right] .
\end{gathered}
$$

It consists of a fictitious kinetic energy for the wave functions, the kinetic energy of the ions, an energy expression composed of the electronic energy functional and the ion-ion interaction energy, and a constraint term that ensures the orthonormality of the electronic states. The Euler-Lagrange equations then lead to the equations of motion for the electronic states

$$
m_{\psi}\left|\ddot{\psi}_{n}\right\rangle=-\frac{1}{f_{n}} \frac{\partial E}{\partial\left\langle\psi_{n}\right|}-\alpha m_{\psi}\left|\dot{\psi}_{n}\right\rangle-\sum_{m}\left|\psi_{m}\right\rangle \frac{\Lambda_{n m}}{f_{n}},
$$

and the ions

$$
m_{\vec{R}} \ddot{\vec{R}}_{i}=-\nabla_{i} E-\gamma m_{\vec{R}} \dot{\vec{R}}_{i}
$$

Note that in both equations friction terms have been added, which are used to dampen out excess kinetic energy during the minimization procedure. $\alpha$ and $\gamma$ are technical friction constants, which are used to control the optimization procedure. In the CP-PAW code the equations of motion (3.159) and (3.160) are integrated with a Verlet algorithm. The energy minimum is reached, when the kinetic energies of the wave functions and of the ions reach zero and no kinetic energy buildup occurs in further time steps.

In the case of a metallic material it is necessary to allow for variable occupancies of the Kohn-Sham states during the simulation [119]. Auxiliary energies for the wave functions are introduced, which follow the real energy levels during the optimization steps in a retarded fashion. The wave function occupancies are computed from these auxiliary energy levels. The auxiliary energy levels approach the real energy levels exponentially. 


\subsection{The projector augmented-wave method}

The projector augmented-wave method [120] is an approach to electronic structure computations that was developed as a generalization of earlier augmentedwave and pseudopotential methods. It provides an efficient set of basis functions for simulations of real materials. Furthermore, it is designed to integrate well into the framework of density functional theory together with the Car-Parrinello molecular dynamics optimization scheme.

The number of electronic degrees of freedom in a real material is typically very large. To keep computational costs in numerical simulations of real materials low, an efficient set of basis function is required. The PAW method divides space into spherical augmentation regions, which are centered around ion nuclei, and the interstitial space. In the interstitial space between the augmentation regions and away from the nuclei, a useful set of basis states are plane waves, which are solutions to the Schrödinger equation in a periodic and/or constant potential. However, close to a nucleus, a spherically symmetric potential dominates and the potentials originating from other nuclei in the vicinity are negligible. There, the wave functions oscillate rapidly and an accurate numerical representation would require a superposition of many plane waves and a fine spatial grid. Hence, inside the augmentation regions the set of atomic wave functions is more efficient.

The PAW method provides a formalism that represents the physically relevant wave functions, commonly called all-electron wave functions, by smooth parts in the interstitial space and in terms of atomic wave functions inside the augmentation regions. Computationally convenient smooth wave functions are used as variables, so-called pseudo wave functions, from which the all-electron wave functions can be retrieved.

The following description of the PAW method is based on the original publication [120] and an introductory guide [121] to the technique.

\subsubsection{Basic formalism}

The basic idea of the PAW method is, to retrieve a full all-electron wave function $\left|\psi_{n}\right\rangle$ from a pseudo wave function $\left|\tilde{\psi}_{n}\right\rangle$ via a linear transformation

$$
\left|\psi_{n}\right\rangle=\hat{\mathcal{T}}\left|\tilde{\psi}_{n}\right\rangle
$$

Because the all-electron wave functions and the pseudo wave functions coincide outside of the augmentations regions, the operator $\hat{\mathcal{T}}$ needs to transform the pseudo wave functions only inside the augmentation regions. Hence, it is of the general form

$$
\hat{\mathcal{T}}=\hat{1}+\sum_{A} \hat{\mathcal{T}}_{A},
$$

where the sum runs over all augmentation regions and where the transformation within the $A$-th augmentation region is given by the transformation operator $\hat{\mathcal{T}}_{A}$. 
Like in the original augmented plane wave method, an augmentation region is dominated by the potential of its central nucleus. A convenient set of basis states inside an augmentation region are the solutions of the Schrödinger equation in a spherically symmetric potential. In the context of the PAW method, these basis functions are commonly referred to as all-electron partial wave functions. They have the form of a product of a spherical harmonic and a radial function, which is found by integration of the radial part of the Schrödinger equation. In the CP-PAW code they are computed on a radial grid at the start of a simulation.

At this point a distinction is made between core and valence states. Core states of an ion are low in energy and less affected by other ions close by. Commonly, for the core states the frozen core approximation is used, which keeps the core states fixed and always occupied during a simulation. In contrast, valence states are affected by the environment of the ion and are subject to change during a simulation. For the simulations in the context of this thesis, the frozen core approximation was used. The number of valence electrons is specified in the simulation setup, from which the core states are determined. Note that the set of all-electron partial wave functions for the valence states is a complete set of states that are orthogonal to the core states.

We proceed with the transformation formalism for the valence states. For an all-electron partial wave function we write $\left|\phi_{A, i}\right\rangle$, where the index $i$ is a combined index for the angular momentum quantum number $l$, the magnetic quantum number $m$, and a label for partial wave functions with the same $l$ and $m$. Each all-electron partial wave function $\left|\phi_{A, i}\right\rangle$ is linked to a pseudo partial wave function $\left|\tilde{\phi}_{A, i}\right\rangle$ via the transformation operator inside the augmentation region $A$,

$$
\left|\phi_{A, i}\right\rangle=\left(\hat{1}+\hat{\mathcal{T}}_{A}\right)\left|\tilde{\phi}_{A, i}\right\rangle .
$$

Outside the augmentation region we have $\left|\phi_{A, i}\right\rangle=\left|\tilde{\phi}_{A, i}\right\rangle$. A pseudo wave function can be represented as a linear combination of the pseudo partial wave functions,

$$
\left|\tilde{\psi}_{n}\right\rangle=\sum_{i}\left|\tilde{\phi}_{A, i}\right\rangle c_{n, A, i}
$$

According to eqs. (3.163) and (3.164), inside the augmentation region $A$, the all-electron wave function is a linear combination of the all-electron partial wave functions,

$$
\left|\psi_{n}\right\rangle=\sum_{i}\left|\phi_{A, i}\right\rangle c_{n, A, i}
$$

where the expansion coefficients $c_{n, A, i}$ are the same as in the expansion of the pseudo wave function (3.164). The combination of eqs. (3.164) and (3.165), leads to the representation of an all-electron wave function in terms of a pseudo wave function and the all-electron and pseudo partial wave functions,

$$
\left|\psi_{n}\right\rangle=\left|\tilde{\psi}_{n}\right\rangle+\sum_{i}\left(\left|\phi_{A, i}\right\rangle-\left|\tilde{\phi}_{A, i}\right\rangle\right) c_{n, A, i}
$$

Since $\hat{\mathcal{T}}$ shall be a linear transformation operator, the coefficients $c_{n, A, i}$ have to be linear in $\left|\tilde{\psi}_{n}\right\rangle$, i. e.

$$
c_{n, A, i}=\left\langle\tilde{p}_{A, i} \mid \tilde{\psi}_{n}\right\rangle,
$$


where $\left\langle\tilde{p}_{A, i}\right|$ is called a projector function. Note that $\left\langle\tilde{p}_{A, i}\right|$ is restricted to the augmentation region $A$. By inserting eq. (3.167) into eq. (3.165) and applying eq. (3.165) recursively, the two properties

$$
\sum_{i}\left|\tilde{\phi}_{A, i}\right\rangle\left\langle\tilde{p}_{A, i}\right|=\hat{1} \quad \text { and } \quad\left\langle\tilde{p}_{A, i} \mid \tilde{\phi}_{A, j}\right\rangle=\delta_{i j}
$$

can be obtained. Furthermore, by inserting eq. (3.167) into eq. (3.166) and taking into account the complete set of augmentation spheres, the transformation operator takes the form

$$
\hat{\mathcal{T}}=\hat{1}+\sum_{A, i}\left(\left|\phi_{A, i}\right\rangle-\left|\tilde{\phi}_{A, i}\right\rangle\right)\left\langle\tilde{p}_{A, i}\right| .
$$

An all-electron core wave function $\left|\psi_{q}^{\text {core }}\right\rangle$ is connected to a pseudo core wave function $\left|\tilde{\psi}_{q}^{\text {core }}\right\rangle$, an all-electron core partial wave function $\left|\phi_{q}^{\text {core }}\right\rangle$, and a pseudo core partial wave function $\left|\tilde{\phi}_{q}^{\text {core }}\right\rangle$ by

$$
\left|\psi_{q}^{\text {core }}\right\rangle=\left|\tilde{\psi}_{q}^{\text {core }}\right\rangle+\left|\phi_{q}^{\text {core }}\right\rangle-\left|\tilde{\phi}_{q}^{\text {core }}\right\rangle
$$

Equation (3.170) is a simple analog to eq. (3.166), with only one all-electron partial wave function corresponding to one all-electron wave function and one pseudo partial wave function corresponding to one pseudo wave function.

\subsubsection{Expectation values, the density, and the total energy}

Within the framework of the PAW method, the pseudo wave functions are the computational variables. However, observables need to be obtained with the physically relevant wave functions. Hence, the transformation operator (3.169) is used in the evaluation of operator expectation values. The expectation value of an operator $\hat{X}$ becomes

$$
\begin{aligned}
\langle\hat{X}\rangle= & \sum_{n} f_{n}\left\langle\psi_{n}|\hat{X}| \psi_{n}\right\rangle+\sum_{q=1}^{N_{\text {core }}}\left\langle\psi_{q}^{\text {core }}|\hat{X}| \psi_{q}^{\text {core }}\right\rangle \\
= & \sum_{n} f_{n}\left\langle\tilde{\psi}_{n}\left|\hat{\mathcal{T}}^{\dagger} \hat{X} \hat{\mathcal{T}}\right| \tilde{\psi}_{n}\right\rangle+\sum_{q=1}^{N_{\text {core }}}\left\langle\psi_{q}^{\text {core }}|\hat{X}| \psi_{q}^{\text {core }}\right\rangle \\
= & \sum_{n} f_{n}\left\langle\tilde{\psi}_{n}|\hat{X}| \tilde{\psi}_{n}\right\rangle+\sum_{q=1}^{N_{\text {core }}}\left\langle\psi_{q}^{\text {core }}|\hat{X}| \psi_{q}^{\text {core }}\right\rangle \\
& +\sum_{n, A, i, j}\left\langle\tilde{p}_{A, j} \mid \tilde{\psi}_{n}\right\rangle f_{n}\left\langle\tilde{\psi}_{n} \mid \tilde{p}_{A, i}\right\rangle\left(\left\langle\phi_{A, i}|\hat{X}| \phi_{A, j}\right\rangle-\left\langle\tilde{\phi}_{A, i}|\hat{X}| \tilde{\phi}_{A, j}\right\rangle\right)
\end{aligned}
$$

The equality in the last step holds for local operators only. For non-local operators a correction term has to be included, see $[120,121]$. For numerical stability, the core partial waves are often included in the last part of eq. (3.173) 
in radial representation, so that the expectation value becomes

$$
\begin{aligned}
& \langle\hat{X}\rangle=\sum_{n} f_{n}\left\langle\tilde{\psi}_{n}|\hat{X}| \tilde{\psi}_{n}\right\rangle+\sum_{q=1}^{N_{\text {core }}}\left\langle\tilde{\phi}_{q}^{\text {core }}|\hat{X}| \tilde{\phi}_{q}^{\text {core }}\right\rangle \\
& +\sum_{A}\left(\sum_{i j} D_{A j i}\left\langle\phi_{A, i}|\hat{X}| \phi_{A, j}\right\rangle+\sum_{\substack{q=1 \\
q \text { in } A}}^{N_{\text {core } A}}\left\langle\phi_{q}^{\text {core }}|\hat{X}| \phi_{q}^{\text {core }}\right\rangle\right) \\
& -\sum_{A}\left(\sum_{i j} D_{A j i}\left\langle\tilde{\phi}_{A, i}|\hat{X}| \tilde{\phi}_{A, j}\right\rangle+\sum_{\substack{q=1 \\
q \text { in } A}}^{N_{\text {core }, A}}\left\langle\tilde{\phi}_{q}^{\text {core }}|\hat{X}| \tilde{\phi}_{q}^{\text {core }}\right\rangle\right),
\end{aligned}
$$

where the matrix

$$
D_{A i j}=\sum_{n}\left\langle\tilde{p}_{A, i} \mid \tilde{\psi}_{n}\right\rangle f_{n}\left\langle\tilde{\psi}_{n} \mid \tilde{p}_{A, j}\right\rangle
$$

was introduced.

An important expectation value that needs to be evaluated during a DFT computation is that of the operator $|\vec{r}\rangle\langle\vec{r}|$, which gives the electron density. It reads

$$
n(\vec{r})=\tilde{n}(\vec{r})+\sum_{A}\left(n_{A}^{1}(\vec{r})-\tilde{n}_{A}^{1}(\vec{r})\right)
$$

where

$$
\begin{gathered}
\tilde{n}(\vec{r})=\sum_{n} f_{n} \tilde{\psi}_{n}^{*}(\vec{r}) \tilde{\psi}_{n}(\vec{r})+\sum_{q=1}^{N_{\text {core }}} \tilde{\phi}_{q}^{\text {core } *}(\vec{r}) \tilde{\phi}_{q}^{\text {core }}(\vec{r}) \\
n_{A}^{1}(\vec{r})=\sum_{i j} D_{A j i} \phi_{A, i}^{*}(\vec{r}) \phi_{A, j}(\vec{r})+\sum_{\substack{q=1 \\
q \text { in } A}}^{N_{\text {core }, A}} \phi_{q}^{\text {core } *}(\vec{r}) \phi_{q}^{\text {core }}(\vec{r}) \\
\tilde{n}_{A}^{1}(\vec{r})=\sum_{i j} D_{A j i} \tilde{\phi}_{A, i}^{*}(\vec{r}) \tilde{\phi}_{A, j}(\vec{r})+\sum_{\substack{\text { core }, A \\
q \text { in } A}}^{\text {core } *}(\vec{r}) \tilde{\phi}_{q}^{\text {core }}(\vec{r})
\end{gathered}
$$

Partial waves of the valence and core states are included in their radial representation, in which they are computed in the simulation setup. The pseudo wave functions are optimized during a simulation and are known in the form of a plane wave expansion. They are also needed to compute the matrix elements $D_{A i j}$.

The total energy is evaluated from eq. (3.174) by inserting the many-electron Hamiltonian. This leads to the total energy

$$
E[\{|\tilde{\psi}\rangle\},\{f\}]=\tilde{E}+\sum_{A}\left(E_{A}^{1}-\tilde{E}_{A}^{1}\right),
$$


where

$$
\begin{aligned}
\tilde{E}[\{|\tilde{\psi}\rangle\},\{f\}]=- & \frac{\hbar^{2}}{2 m} \sum_{n}\left\langle\tilde{\psi}_{n}\left|\nabla^{2}\right| \tilde{\psi}_{n}\right\rangle+\int \mathrm{d} \vec{r} \bar{v}(\vec{r}) \tilde{n}(\vec{r}) \\
& +\frac{e^{2}}{8 \pi \varepsilon_{0}} \iint \mathrm{d} \vec{r} \mathrm{~d} \vec{r}^{\prime} \frac{[\tilde{n}(\vec{r})+\tilde{Z}(\vec{r})]\left[\tilde{n}\left(\vec{r}^{\prime}\right)+\tilde{Z}\left(\vec{r}^{\prime}\right)\right]}{\left|\vec{r}-\vec{r}^{\prime}\right|} \\
& +\int \mathrm{d} \vec{r} \varepsilon_{\mathrm{xc}}[\tilde{n}](\vec{r}) \tilde{n}(\vec{r}) \\
E_{A}^{1}[\{|\tilde{\psi}\rangle\},\{f\}]=- & \frac{\hbar^{2}}{2 m} \sum_{i j} D_{A j i}\left\langle\phi_{A, i}\left|\nabla^{2}\right| \phi_{A, j}\right\rangle-\frac{\hbar^{2}}{2 m} \sum_{q=1}^{N_{\text {core }, A}}\left\langle\phi_{q}^{\text {core }}\left|\nabla^{2}\right| \phi_{q}^{\text {core }}\right\rangle \\
& +\frac{e^{2}}{8 \pi \varepsilon_{0}} \iint \mathrm{d} \vec{r} \mathrm{~d} \vec{r}^{\prime} \frac{\left[n_{A}^{1}(\vec{r})+Z_{A}(\vec{r})\right]\left[n_{A}^{1}\left(\vec{r}^{\prime}\right)+Z_{A}\left(\vec{r}^{\prime}\right)\right]}{\left|\vec{r}-\vec{r}^{\prime}\right|} \\
& +\int \mathrm{d} \vec{r} \varepsilon_{\mathrm{xc}}\left[n_{A}^{1}\right](\vec{r}) n_{A}^{1}(\vec{r}) \\
& +\frac{\hbar^{2}}{2 m} \sum_{i j} D_{A j i}\left\langle\tilde{\phi}_{A, i}\left|\nabla^{2}\right| \tilde{\phi}_{A, j}\right\rangle+\int \mathrm{d} \vec{r} \bar{v}(\vec{r}) \tilde{n}_{A}^{1}(\vec{r}) \\
& +\frac{e^{2}}{8 \pi \varepsilon_{0}} \iint \mathrm{d} \vec{r} \mathrm{~d} \vec{r}^{\prime} \frac{\left[\tilde{n}_{A}^{1}(\vec{r})+\tilde{Z}_{A}(\vec{r})\right]\left[\tilde{n}_{A}^{1}\left(\vec{r}^{\prime}\right)+\tilde{Z}_{A}\left(\vec{r}^{\prime}\right)\right]}{\left|\vec{r}-\vec{r}^{\prime}\right|} \\
& +\int \mathrm{d} \vec{r} \varepsilon_{\mathrm{xc}}\left[\tilde{n}_{A}^{1}\right](\vec{r}) \tilde{n}_{A}^{1}(\vec{r}) .
\end{aligned}
$$

$Z_{A}(\vec{r})$ is the point charge density of the nucleus, whose self interaction needs to be subtracted from eq. (3.182). The compensation charge density $\tilde{Z}(\vec{r})=$ $\sum_{A} \tilde{Z}_{A}(\vec{r})$ is constructed such that the electrostatic multipole moments of the charge densities of the one-center expansion terms $n_{A}^{1}(\vec{r})+Z_{A}(\vec{r})-\tilde{n}_{A}^{1}(\vec{r})-\tilde{Z}_{A}(\vec{r})$ vanish.

In eqs. (3.181) and (3.183), the potential $\bar{v}$ is added by exploiting that in each augmentation sphere $A$ a zero term of the form

$$
\sum_{n} f_{n}\left\langle\tilde{\psi}_{n}\left|\left[\hat{Y}-\sum_{i j}\left|\tilde{p}_{A, i}\right\rangle\left\langle\tilde{\phi}_{A, i}|\hat{Y}| \tilde{\phi}_{A, j}\right\rangle\left\langle\tilde{p}_{A, j}\right|\right]\right| \tilde{\psi}_{n}\right\rangle=0
$$

can be inserted. Thus $\bar{v}$ has to be localized in an augmentation sphere, but can be chosen arbitrarily otherwise. It is used to improve the partial wave convergence. Note furthermore that the kinetic energy due to the pseudo core states in eqs. (3.181) and (3.183) cancel each other.

\subsection{The density of states}

The density of states (DOS) represents the distribution of one-particle states along the energy axis. It contains information about the material at hand, e.g. 
electronic transport and magnetic properties, and is an important quantity in DFT material studies. Moreover, the DOS is also a technical quantity that allows to switch from an integration over the degrees of freedom in a system to an energy integration. In the following this will be illustrated for the expectation value of an observable $\hat{A}$. We use the Fermi distribution function

$$
f_{\beta \mu}(\varepsilon)=[1+\exp (\beta(\varepsilon-\mu))]^{-1},
$$

where $\beta=\left(k_{\mathrm{B}} T\right)^{-1}$ is the inverse temperature, $k_{\mathrm{B}}$ is the Boltzmann constant and $\mu$ is the chemical potential. Furthermore, because DFT simulations are usually performed in reciprocal space, a dependence on the reciprocal vector $\vec{k}$ is included in the following. The volume of the reciprocal unit cell is denoted $V_{G}$. With one-particle basis states $\left|\psi_{n}(\vec{k})\right\rangle$, their respective occupancy $f_{n}(\vec{k})$, and matrix elements $A_{n}(\vec{k})=\left\langle\psi_{n}(\vec{k})|\hat{A}| \psi_{n}(\vec{k})\right\rangle$, the expectation value of an observable $\hat{A}$ reads [56]

$$
\begin{aligned}
\langle\hat{A}\rangle & =\frac{1}{V_{G}} \sum_{n} \int \mathrm{d} \vec{k} f_{n}(\vec{k}) A_{n}(\vec{k}) \\
& =\frac{1}{V_{G}} \sum_{n} \int \mathrm{d} \vec{k} f_{\beta \mu}\left(\varepsilon_{n}(\vec{k})\right) A_{n}(\vec{k}) \\
& =\frac{1}{V_{G}} \sum_{n} \int \mathrm{d} \vec{k} \int \mathrm{d} \varepsilon \delta\left(\varepsilon-\varepsilon_{n}(\vec{k})\right) f_{\beta \mu}\left(\varepsilon_{n}(\vec{k})\right) A_{n}(\vec{k}) \\
& =\int \mathrm{d} \varepsilon f_{\beta \mu}(\varepsilon) \underbrace{\frac{1}{V_{G}} \sum_{n} \int \mathrm{d} \vec{k} \delta\left(\varepsilon-\varepsilon_{n}(\vec{k})\right) A_{n}(\vec{k})}_{D^{A}(\varepsilon)} \\
& =\int \mathrm{d} \varepsilon f_{\beta \mu}(\varepsilon) D^{A}(\varepsilon) .
\end{aligned}
$$

Here,

$$
D^{A}(\varepsilon)=\frac{1}{V_{G}} \sum_{n} \int \mathrm{d} \vec{k} \delta\left(\varepsilon-\varepsilon_{n}(\vec{k})\right) A_{n}(\vec{k})
$$

is the DOS, weighted by the matrix elements $A_{n}(\vec{k})$ of operator $\hat{A}$. Moreover, insertion of the unity operator

$$
\hat{\mathbb{1}}=\frac{1}{V_{G}} \sum_{n} \int \mathrm{d} \vec{k}\left|\psi_{n}(\vec{k})\right\rangle\left\langle\psi_{n}(\vec{k})\right|
$$

leads to

$$
\begin{aligned}
D^{A}(\varepsilon)=\frac{1}{V_{G}} & \sum_{n} \int \mathrm{d} \vec{k} \delta\left(\varepsilon-\varepsilon_{n}(\vec{k})\right) \times \\
& \times\left\langle\psi_{n}(\vec{k})\left|\hat{A}\left[\frac{1}{V_{G}} \sum_{m} \int \mathrm{d} \vec{k}^{\prime}\left|\psi_{m}\left(\vec{k}^{\prime}\right)\right\rangle\left\langle\psi_{m}\left(\vec{k}^{\prime}\right)\right|\right]\right| \psi_{n}(\vec{k})\right\rangle
\end{aligned}
$$




$$
\begin{aligned}
& =\frac{1}{V_{G}} \sum_{m} \int \mathrm{d} \vec{k}^{\prime}\left\langle\psi_{m}\left(\vec{k}^{\prime}\right)\right| \times \\
& \quad \times\left[\frac{1}{V_{G}} \sum_{n} \int \mathrm{d} \vec{k}\left|\psi_{n}(\vec{k})\right\rangle \delta\left(\varepsilon-\varepsilon_{n}(\vec{k})\right)\left\langle\psi_{n}(\vec{k})\right| \hat{A}\right]\left|\psi_{m}\left(\vec{k}^{\prime}\right)\right\rangle \\
& =\operatorname{Tr}[\underbrace{\left.\frac{1}{V_{G}} \sum_{n} \int \mathrm{d} \vec{k}\left|\psi_{n}(\vec{k})\right\rangle \delta\left(\varepsilon-\varepsilon_{n}(\vec{k})\right)\left\langle\psi_{n}(\vec{k})\right| \hat{A}\right]}_{\hat{D}(\varepsilon)}
\end{aligned}
$$

where

$$
\hat{D}(\varepsilon)=\frac{1}{V_{G}} \sum_{n} \int \mathrm{d} \vec{k}\left|\psi_{n}(\vec{k})\right\rangle \delta\left(\varepsilon-\varepsilon_{n}(\vec{k})\right)\left\langle\psi_{n}(\vec{k})\right|
$$

is the density of states operator.

\section{Total density of states}

In DFT the total density of states is the DOS coming from the full set of Kohn-Sham states. It is obtained as the trace of the DOS operator,

$$
D_{\text {tot }}(\varepsilon)=\operatorname{Tr}[\hat{D}(\varepsilon)]=\frac{1}{V_{G}} \sum_{n} \int \mathrm{d} \vec{k} \delta\left(\varepsilon-\varepsilon_{n}(\vec{k})\right) .
$$

Equation (3.197) is also the energy derivative of the total electron number

$$
N_{\text {tot }}(\varepsilon)=\frac{1}{V_{G}} \sum_{n} \int \mathrm{d} \vec{k} \Theta\left(\varepsilon-\varepsilon_{n}(\vec{k})\right) .
$$

\section{Projected density of states}

Often a representation of the DOS is desirable in which the contributions of local orbitals of the atoms in the unit cell are resolved. In this regard, it is useful to decompose a Kohn-Sham state into local orbitals $\left|\chi_{\alpha, \vec{R}}\right\rangle$, with associated projectors $\left|\pi_{\alpha, \vec{R}}\right\rangle$ :

$$
\left|\psi_{n}(\vec{k})\right\rangle=\sum_{\alpha \vec{R}}\left|\chi_{\alpha, \vec{R}}\right\rangle\left\langle\pi_{\alpha, \vec{R}} \mid \psi_{n}(\vec{k})\right\rangle
$$

where $\vec{R}$ denotes a lattice vector and $\alpha$ labels the local orbitals of the unit cell. Equation (3.188) then becomes

$$
\langle\hat{A}\rangle=\sum_{\alpha \alpha^{\prime} \vec{R} \vec{R}^{\prime}}\left\langle\chi_{\alpha, \vec{R}}|\hat{A}| \chi_{\alpha^{\prime}, \vec{R}^{\prime}}\right\rangle \int \mathrm{d} \varepsilon f_{\beta \mu}(\varepsilon) D_{\alpha \alpha^{\prime} \vec{R} \vec{R}^{\prime}}(\varepsilon),
$$


where

$$
\begin{aligned}
D_{\alpha \alpha^{\prime} \vec{R} \vec{R}^{\prime}}(\varepsilon) & =\left\langle\pi_{\alpha^{\prime} \vec{R}^{\prime}}|\hat{D}(\varepsilon)| \pi_{\alpha \vec{R}}\right\rangle \\
& =\frac{1}{V_{G}} \sum_{n} \int \mathrm{d} \vec{k}\left\langle\pi_{\alpha^{\prime} \vec{R}^{\prime}} \mid \psi_{n}(\vec{k})\right\rangle \delta\left(\varepsilon-\varepsilon_{n}(\vec{k})\right)\left\langle\psi_{n}(\vec{k}) \mid \pi_{\alpha \vec{R}}\right\rangle .
\end{aligned}
$$

The diagonal elements of $D_{\alpha \alpha^{\prime} \vec{R} \vec{R}^{\prime}}(\varepsilon)$ are the projected density of states of the orbital $\left|\chi_{\alpha \vec{R}}\right\rangle$,

$$
D_{\alpha \vec{R}}(\epsilon)=\left\langle\pi_{\alpha \vec{R}}|\hat{D}(\varepsilon)| \pi_{\alpha \vec{R}}\right\rangle=\frac{1}{V_{G}} \sum_{n} \int \mathrm{d} \vec{k} \delta\left(\varepsilon-\varepsilon_{n}(\vec{k})\right)\left|\left\langle\pi_{\alpha \vec{R}} \mid \psi_{n}(\vec{k})\right\rangle\right|^{2} .
$$

\section{Local density of states}

Two of the most important quantities of interest in material research are the electron density and the density of states. While the electron density provides information about the spatial distribution of electronic states, the density of states holds information about the energetic distribution of states in a material. The local density of states (LDOS) can be viewed as a combination of the two. It is defined as

$$
D_{\mathrm{loc}}(\vec{r}, \varepsilon)=\sum_{n}\left|\psi_{n}(\vec{r})\right|^{2} \delta\left(\varepsilon-\varepsilon_{n}\right)
$$

and thus is a measure for the spatial electronic density emerging only from those states that have the energy $\varepsilon$. The LDOS is especially useful in surface studies, where the spatial extent of surface states into the vacuum is of interest. 


\subsection{Scanning tunneling microscopy}

A family of experimental techniques that are nowadays commonly used in surface studies of solid state materials is known under the term scanning probe microscopy (SPM). In SPM experiments, a probe scans the surface of a sample to gather data about the surface structure in real space which is then used for real space surface imaging. One of the most widely known SPM techniques is the scanning tunneling microscopy (STM). First successful STM surface imaging experiments with atomic resolution have been conducted in the early 1980 s $[122,123]$ and contributed significantly to the development of the family of SPM techniques.

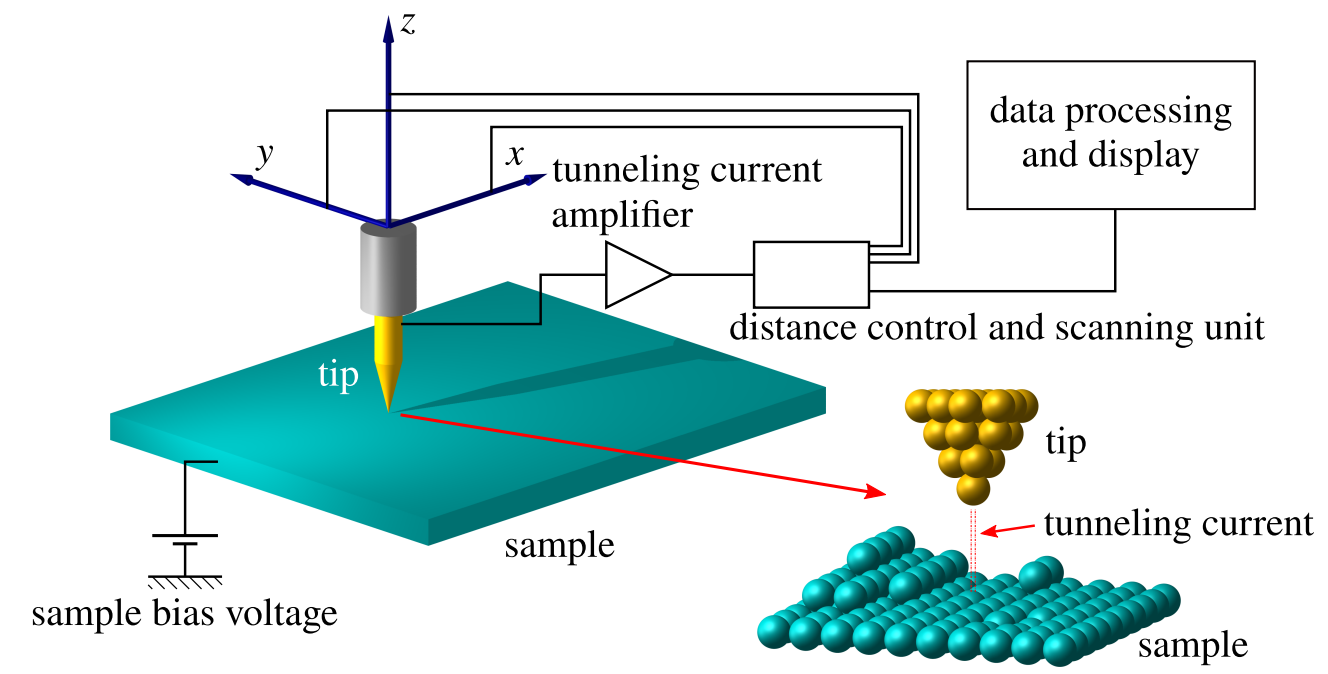

Figure 3.1: Schematic diagram of an STM. The sample is scanned in the $x y$ plane. The tip height above the surface is denoted by the $z$ coordinate. The distance control and scanning unit accepts inputs from the STM operator to move the tip along the three spatial coordinates and also closes the feedback loop for tip height adjustments in reaction to the tunneling current.

STM operation relies on the tunnel effect, which leads to the formation of a tunneling current between a sample and a probing tip, where the tip is positioned at a small distance above the sample surface, see fig. 3.1. A bias voltage between tip and sample is used to tune the tunneling current. With the bias voltage polarity the direction of the tunneling current is chosen, i.e. electrons tunneling from the sample to the tip or vice versa. The initial tip height for a measurement can be set by first specifying a bias voltage and second selecting a tunneling current set point. When the specified set point for the tunneling current is reached, the tip's approach to the sample is stopped. Although the exact distance between the tip and the sample cannot be easily determined, it is usually at $\sim 1 \mathrm{~nm}$ [122]. When the tip is positioned close to the surface and a tunneling current is formed, the sample surface is scanned by moving the tip along the $x$ and $y$ coordinates. The tip is connected to a feedback loop that allows for height adjustments in reaction to changes of the 
tunneling current while scanning the sample. To achieve a spatial resolution of $\sim 2 \AA$ [124] in the $x y$ plane, a sharp tip is necessary, preferably with only one atom at the tip apex.

There are two main modes of operation of an STM: the constant current mode and the constant height mode.

- STM operation in the constant current mode aims to keep the tunneling current constant. While scanning the sample surface, the feedback loop adjusts he tip height in reaction to changes of the tunneling current to keep the tunneling current constant. The spatially resolved changes of the tip height with respect to the initial reference height are recorded and displayed as a constant current topography (CCT) image of the surface.

- In the constant height mode, the feedback loop is deactivated and the tip height is kept fixed during a surface scan. While scanning the sample surface the tunneling current is recorded. As a result, spatially resolved data for the tunneling current is obtained in the plane perpendicular to the sample and displayed as an image. A disadvantage of the constant height mode is that the tip might make contact with the surface, for example when the scanning plane is not exactly parallel to the surface plane or when there are steps occurring on the sample surface.

In modern STM experiments the constant current mode is usually the preferred mode of operation. Today's feedback loops allow for tip height adjustment of $\sim 1 \AA$ Å during surface imaging.

It is important to note that the STM image of a material's surface often shows a pattern that is not a direct representation of the arrangement of the underlying surface atoms. This is because in an STM topography, the electronic states of the surface are mapped rather than the atomic nuclei. However, the atomic structure can sometimes be reconstructed from the STM data after comparison with data from theoretical calculations.

Both STM measurement modes rely on the measurement of the tunneling current between tip and sample, which is a central quantity in STM experiments. The tunneling current depends on the electronic states of the sample as well as the tip, so that a theory for the STM in principle needs to account for both, the sample and the tip states. In this regard, the most widely used approach is based on Bardeen's tunneling theory [125], which was originally developed to describe tunneling in a metal-insulator-metal sandwich structure. For practical applications, usually simplifications to Bardeen's theory are introduced because the tip states are not known exactly, and neither are the transition amplitudes between the sample and tip states. We use the Tersoff-Hamann approximation $[126,127]$, which leads to a simplification of the tunneling-current expression. In the following sections, after a brief recap of the basics of quantum tunneling theory, the approach via Bardeen's tunneling theory, combined with the TersoffHamann approximation, is discussed in detail. 


\subsubsection{Tunneling theory}

A very basic model for the STM is a square potential representing the vacuum, which separates two zero-potential regions representing the sample and the tip:

$$
V_{\text {barrier }}(z)=\left\{\begin{array}{ll}
V_{\mathrm{vac}}, & \text { for } z_{0} \leq z \leq z_{1} \\
0, & \text { otherwise }
\end{array} .\right.
$$

From quantum tunneling theory it is well known that the sample wave function in the vacuum region has the solution

$$
\psi(z)=\psi\left(z_{0}\right) e^{-\kappa\left(z-z_{0}\right)}
$$

where

$$
\kappa=\frac{\sqrt{2 m\left(V_{\mathrm{vac}}-E\right)}}{\hbar} .
$$

This describes the decay of the initial sample wave function $\psi\left(z_{0}\right)$ in positive $z$ direction. In a material, the electronic states are filled up to the Fermi level $E_{\mathrm{F}}$. The minimal energy required to remove an electron from the material is known as the work function,

$$
\phi=V_{\mathrm{vac}}-E_{\mathrm{F}}
$$

Using eq. (3.208), the probability to find an electron with energy $E$ on the other side of the vacuum barrier of width $\Delta z=z_{1}-z_{0}$ can be expressed as

$$
P\left(z_{1}, E\right)=\left|\psi\left(z_{0}\right)\right|^{2} e^{-2 \kappa \Delta z}=\left|\psi\left(z_{0}\right)\right|^{2} e^{-\sqrt{8 m} \sqrt{\phi-\left(E-E_{\mathrm{F}}\right)} \Delta z / \hbar} .
$$

The decay constant $\kappa$ is smaller, the higher the electron energy. Hence, the tunneling probability is larger the closer an electron's energy is to the Fermi level. For states that lie in close vicinity to the Fermi level, $E \approx E_{\mathrm{F}}$, we find

$$
P\left(z_{1}, E=E_{\mathrm{F}}\right)=\left|\psi\left(z_{0}\right)\right|^{2} e^{-2 \kappa \Delta z}=\left|\psi\left(z_{0}\right)\right|^{2} e^{-2 \sqrt{2 m} \sqrt{\phi} \Delta z / \hbar} .
$$

\subsubsection{Theory of the STM from perturbation theory}

In this and the following sections, we present a perturbative approach to the STM, which involves both the tip and the sample. We follow along the texts of Chen [124] and Lounis [128]. The tunneling theory of Bardeen [125] as well as the Tersoff-Hamann approximation $[126,127]$ will be used to obtain a relation between the tunneling current and the local density of states (LDOS) of the sample at the position of the tip.

We start with a sample system and a tip system that are independent while the tip (T) is far above the surface (S). Their physics is governed by the timeindependent Schrödinger equations,

$$
\left(\hat{T}+\hat{V}_{\mathrm{S}}\right)\left|\psi_{\mu}^{\mathrm{S}}\right\rangle=E_{\mu}^{\mathrm{S}}\left|\psi_{\mu}^{\mathrm{S}}\right\rangle
$$


and

$$
\left(\hat{T}+\hat{V}_{\mathrm{T}}\right)\left|\psi_{v}^{\mathrm{T}}\right\rangle=E_{v}^{\mathrm{T}}\left|\psi_{v}^{\mathrm{T}}\right\rangle
$$

respectively. $\hat{T}$ is the kinetic energy operator, $\hat{V}_{\mathrm{S}}$ is the potential of the sample, and $\hat{V}_{\mathrm{T}}$ is the potential of the tip. Furthermore, $\left|\psi_{\mu}^{\mathrm{S}}\right\rangle$ and $\left|\psi_{\nu}^{\mathrm{T}}\right\rangle$ are the wave functions for a state $\mu$ of the sample and a state $v$ of the tip. Their energies are $E_{\mu}^{\mathrm{S}}$ and $E_{\nu}^{\mathrm{T}}$, respectively.

When the tip approaches the surface, a combined system of tip and surface is created and a state in the combined system obeys the time evolution according to the time-dependent Schrödinger equation

$$
i \hbar \frac{\partial}{\partial t}\left|\Psi_{\mu}(t)\right\rangle=\left(\hat{T}+\hat{V}_{\mathrm{S}}+\hat{V}_{\mathrm{T}}^{\mathrm{ad}}(t)\right)\left|\Psi_{\mu}(t)\right\rangle
$$

In this case, let $\left|\Psi_{\mu}(t)\right\rangle$ be a state which evolved from a surface state $\left|\psi_{\mu}^{\mathrm{S}}\right\rangle$ after the tip potential entered the surface system. The tip potential itself is treated perturbatively as a potential that is switched on adiabatically, $\hat{V}_{\mathrm{T}}^{\mathrm{ad}}(t)=\hat{V}_{\mathrm{T}} e^{\eta t / \hbar}$ [128], i. e. $\eta$ is a small real number. This is a reasonable assumption, since the speed of the tip approaching the sample is small compared to the velocity of the electrons in the sample, which adjust to the changing tip potential on a very small time scale.

From the foundations of time-dependent perturbation theory it is well known that an initial state - in this case $\left|\psi_{\mu}^{\mathrm{S}}\right\rangle$ - under the influence of a small timedependent potential evolves according to

$$
\left|\Psi_{\mu}(t)\right\rangle=e^{-i \hat{H}_{0}^{\mathrm{S}} t / \hbar} \hat{U}\left(t, t_{0}\right) e^{i \hat{H}_{0}^{\mathrm{S}} t_{0} / \hbar}\left|\psi_{\mu}^{\mathrm{S}}\left(t_{0}\right)\right\rangle,
$$

where $\hat{H}_{0}^{\mathrm{S}}=\hat{T}+\hat{V}_{\mathrm{S}}$ is the Hamiltonian of the unperturbed sample system. The time evolution operator $\hat{U}\left(t, t_{0}\right)$ in the following will be split into its zero order part and higher order terms which are combined into an operator $\hat{U}^{\prime}\left(t, t_{0}\right)$ : $\hat{U}\left(t, t_{0}\right)=1+\hat{U}^{\prime}\left(t, t_{0}\right)$. Then, the state $\left|\Psi_{\mu}(t)\right\rangle$ can be expanded in terms of the initial sample and tip states:

$$
\begin{aligned}
\left|\Psi_{\mu}(t)\right\rangle & =e^{-i E_{\mu}^{\mathrm{S}}\left(t-t_{0}\right) / \hbar}\left|\psi_{\mu}^{\mathrm{S}}\left(t_{0}\right)\right\rangle+e^{-i \hat{H}_{0}^{\mathrm{S}} t / \hbar} \hat{U}^{\prime}\left(t, t_{0}\right) e^{i \hat{H}_{0}^{\mathrm{S}} t_{0} / \hbar}\left|\psi_{\mu}^{\mathrm{S}}\left(t_{0}\right)\right\rangle \\
& =e^{-i E_{\mu}^{\mathrm{S}}\left(t-t_{0}\right) / \hbar}\left|\psi_{\mu}^{\mathrm{S}}\left(t_{0}\right)\right\rangle+\sum_{v}\left|\psi_{v}^{\mathrm{T}}\left(t_{0}\right)\right\rangle e^{-i E_{v}^{\mathrm{T}}\left(t-t_{0}\right) / \hbar} b_{v}\left(t, t_{0}\right),
\end{aligned}
$$

where $b_{v}\left(t, t_{0}\right)=\left\langle\psi_{v}^{\mathrm{T}}(t)\left|e^{-i \hat{H}_{0}^{\mathrm{S}} t / \hbar} \hat{U}^{\prime}\left(t, t_{0}\right) e^{i \hat{H}_{0}^{\mathrm{S}} t_{0} / \hbar}\right| \psi_{\mu}^{\mathrm{S}}\left(t_{0}\right)\right\rangle$. In the step from eq. (3.215) to eq. (3.216) the completeness relation w. r. t. the tip states $\left|\psi_{v}^{\mathrm{T}}(t)\right\rangle$ was exploited, where $\left|\psi_{v}^{\mathrm{T}}(t)\right\rangle$ is a time-evolved state of the initial tip state under the time-independent tip Hamiltonian, $\left|\psi_{v}^{\mathrm{T}}(t)\right\rangle=\left|\psi_{v}^{\mathrm{T}}\left(t_{0}\right)\right\rangle e^{-i E_{v}^{\mathrm{T}}\left(t-t_{0}\right) / \hbar}$. 
Inserting eq. (3.216) into eq. (3.213) yields

$$
\begin{aligned}
& i \hbar \frac{\partial}{\partial t}\left|\Psi_{\mu}(t)\right\rangle=E_{\mu}^{S} e^{-i E_{\mu}^{S}\left(t-t_{0}\right) / \hbar}\left|\psi_{\mu}^{\mathrm{S}}\left(t_{0}\right)\right\rangle \\
& +\sum_{v} E_{v}^{\mathrm{T}} e^{-i E_{v}^{\mathrm{T}}\left(t-t_{0}\right) / \hbar} b_{v}\left(t, t_{0}\right)\left|\psi_{v}^{\mathrm{T}}\left(t_{0}\right)\right\rangle \\
& +i \hbar \sum_{v} e^{-i E_{v}^{\mathrm{T}}\left(t-t_{0}\right) / \hbar} \frac{\partial b_{v}\left(t, t_{0}\right)}{\partial t}\left|\psi_{v}^{\mathrm{T}}\left(t_{0}\right)\right\rangle \\
& \stackrel{!}{=}\left(\hat{T}+\hat{V}_{\mathrm{S}}+\hat{V}_{\mathrm{T}} e^{\eta t / \hbar}\right)\left|\Psi_{\mu}(t)\right\rangle \\
& =e^{-i E_{\mu}^{S}\left(t-t_{0}\right) / \hbar}\left(E_{\mu}^{S}+\hat{V}_{\mathrm{T}} e^{\eta t / \hbar}\right)\left|\psi_{\mu}^{\mathrm{S}}\left(t_{0}\right)\right\rangle \\
& +\sum_{v} b_{v}\left(t, t_{0}\right) e^{-i E_{v}^{\mathrm{T}}\left(t-t_{0}\right) / \hbar}\left(E_{v}^{\mathrm{T}}+\hat{V}_{\mathrm{S}}\right)\left|\psi_{v}^{\mathrm{T}}\left(t_{0}\right)\right\rangle \\
& +\sum_{v} b_{v}\left(t, t_{0}\right) e^{-i E_{v}^{\mathrm{T}}\left(t-t_{0}\right) / \hbar} \hat{V}_{\mathrm{T}}\left(e^{\eta t / \hbar}-1\right)\left|\psi_{v}^{\mathrm{T}}\left(t_{0}\right)\right\rangle \text {. }
\end{aligned}
$$

Because $\eta$ is small, $e^{\eta t / \hbar}-1$ is small. Thus, the last term in eq. (3.219) is neglected in the following. Collecting the remaining terms gives

$$
\begin{aligned}
i \hbar \sum_{v} e^{-i E_{\nu}^{\mathrm{T}}\left(t-t_{0}\right) / \hbar} \frac{\partial b_{v}\left(t, t_{0}\right)}{\partial t}\left|\psi_{v}^{\mathrm{T}}\left(t_{0}\right)\right\rangle= & \hat{V}_{\mathrm{T}}\left|\psi_{\mu}^{\mathrm{S}}\left(t_{0}\right)\right\rangle e^{-i E_{\mu}^{S}\left(t-t_{0}\right) / \hbar} e^{\eta t / \hbar} \\
& +\sum_{v} b_{v}\left(t, t_{0}\right) e^{-i E_{v}^{\mathrm{T}}\left(t-t_{0}\right) / \hbar} \hat{V}_{\mathrm{S}}\left|\psi_{v}^{\mathrm{T}}\left(t_{0}\right)\right\rangle .
\end{aligned}
$$

Next, let $\left\langle\psi_{\kappa}^{\mathrm{T}}\left(t_{0}\right)\right|$ act from the left to obtain

$$
\begin{aligned}
i \hbar \frac{\partial b_{\kappa}\left(t, t_{0}\right)}{\partial t}=\langle & \left.\psi_{\kappa}^{\mathrm{T}}\left(t_{0}\right)\left|\hat{V}_{\mathrm{T}}\right| \psi_{\mu}^{\mathrm{S}}\left(t_{0}\right)\right\rangle e^{-i\left(E_{\mu}^{\mathrm{S}}-E_{\kappa}^{\mathrm{T}}+i \eta\right) t / \hbar} e^{i\left(E_{\mu}^{\mathrm{S}}-E_{\kappa}^{\mathrm{T}}\right) t_{0} / \hbar} \\
& +\sum_{v} b_{v}\left(t, t_{0}\right) e^{-i\left(E_{\nu}^{\mathrm{T}}-E_{\kappa}^{\mathrm{T}}\right)\left(t-t_{0}\right) / \hbar}\left\langle\psi_{\kappa}^{\mathrm{T}}\left(t_{0}\right)\left|\hat{V}_{\mathrm{S}}\right| \psi_{v}^{\mathrm{T}}\left(t_{0}\right)\right\rangle .
\end{aligned}
$$

Performing a time integration yields

$$
\begin{aligned}
b_{\kappa}\left(t, t_{0}\right)= & \frac{1}{E_{\mu}^{\mathrm{S}}-E_{\kappa}^{\mathrm{T}}+i \eta}\left\langle\psi_{\kappa}^{\mathrm{T}}\left(t_{0}\right)\left|\hat{V}_{\mathrm{T}}\right| \psi_{\mu}^{\mathrm{S}}\left(t_{0}\right)\right\rangle \times \\
& \times\left(e^{-i\left(E_{\mu}^{\mathrm{S}}-E_{\kappa}^{\mathrm{T}}+i \eta\right) t / \hbar} e^{i\left(E_{\mu}^{\mathrm{S}}-E_{\kappa}^{\mathrm{T}}\right) t_{0} / \hbar}-e^{\eta t_{0} / \hbar}\right) \\
& +\int_{t_{0}}^{t} \mathrm{~d} t^{\prime} \sum_{\nu} b_{\nu}\left(t^{\prime}, t_{0}\right) e^{-i\left(E_{\nu}^{\mathrm{T}}-E_{\kappa}^{\mathrm{T}}\right)\left(t^{\prime}-t_{0}\right) / \hbar}\left\langle\psi_{\kappa}^{\mathrm{T}}\left(t_{0}\right)\left|\hat{V}_{\mathrm{S}}\right| \psi_{\nu}^{\mathrm{T}}\left(t_{0}\right)\right\rangle .
\end{aligned}
$$

Note that $b_{\kappa}\left(t_{0}, t_{0}\right)=0$ since $U^{\prime}\left(t_{0}, t_{0}\right)=0$. Furthermore, the second part of 
the first term is zero for $t_{0} \rightarrow-\infty$, i. e. before the tip enters the sample system. In the following, we restrict ourselves to first order perturbation theory and neglect the last term. In the limit $t_{0} \rightarrow-\infty$ the remainder reads

$$
b_{\kappa}\left(t, t_{0}\right) \approx \frac{1}{E_{\mu}^{\mathrm{S}}-E_{\kappa}^{\mathrm{T}}+i \eta}\left\langle\psi_{\kappa}^{\mathrm{T}}\left(t_{0}\right)\left|\hat{V}_{\mathrm{T}}\right| \psi_{\mu}^{\mathrm{S}}\left(t_{0}\right)\right\rangle e^{-i\left(E_{\mu}^{\mathrm{S}}-E_{\kappa}^{\mathrm{T}}+i \eta\right) t / \hbar} e^{i\left(E_{\mu}^{\mathrm{S}}-E_{\kappa}^{\mathrm{T}}\right) t_{0} / \hbar} .
$$

Inserting this result into eq. (3.216), the overlap amplitude of an initial tip state $\left|\psi_{\kappa}^{\mathrm{T}}\right\rangle$ and the wave function $\left|\Psi_{\mu}(t)\right\rangle$ of the tip-sample system becomes

$$
\left|\left\langle\psi_{\kappa}^{\mathrm{T}} \mid \Psi_{\mu}(t)\right\rangle\right|^{2}=|b(t,-\infty)|^{2}=\frac{e^{2 \eta t / \hbar}}{\left(E_{\mu}^{\mathrm{S}}-E_{\kappa}^{\mathrm{T}}\right)^{2}+\eta^{2}}\left|M_{\mu \kappa}\right|^{2},
$$

where

$$
M_{\mu \kappa}=\left\langle\psi_{\kappa}^{\mathrm{T}}(-\infty)\left|\hat{V}_{\mathrm{T}}\right| \psi_{\mu}^{\mathrm{S}}(-\infty)\right\rangle .
$$

The time derivative of eq. (3.224) gives the transition amplitude of an initial surface state to a tip state,

$$
\begin{aligned}
P_{\mu \kappa}(t) & =\frac{\mathrm{d}}{\mathrm{d} t}\left|\left\langle\psi_{\kappa}^{\mathrm{T}} \mid \Psi_{\mu}(t)\right\rangle\right|^{2} \\
& =\frac{2 \eta}{\hbar} \frac{e^{2 \eta t / \hbar}}{\left(E_{\mu}^{\mathrm{S}}-E_{\kappa}^{\mathrm{T}}\right)^{2}+\eta^{2}}\left|M_{\mu \kappa}\right|^{2} \\
& \stackrel{\eta \rightarrow 0}{\longrightarrow} \frac{2 \pi}{\hbar}\left|M_{\mu \kappa}\right|^{2} \delta\left(E_{\mu}^{\mathrm{S}}-E_{\kappa}^{\mathrm{T}}\right)
\end{aligned}
$$

where the representation of the $\delta$-distribution, $\delta(x)=\lim _{\eta \rightarrow 0} \frac{1}{\pi} \frac{\eta}{x^{2}+\eta^{2}}$, has been used. Hence, as the result, we obtain Fermi's golden rule.

\subsubsection{The tunneling current}

With the transition amplitude (3.228) an expression for the tunneling current can be formulated as follows. The tunneling current from the sample to the tip is given by the sum of the transitions amplitudes of all possible transitions, times the electron charge:

$$
I_{\mathrm{S} \rightarrow \mathrm{T}}=\frac{4 \pi e}{\hbar} \sum_{\mu \nu} f\left(E_{\mu}^{\mathrm{S}}-E_{\mathrm{F}}^{\mathrm{S}}\right)\left[1-f\left(E_{v}^{\mathrm{T}}-E_{\mathrm{F}}^{\mathrm{T}}\right)\right]\left|M_{\mu \nu}\right|^{2} \delta\left(E_{v}^{\mathrm{T}}-E_{\mu}^{\mathrm{S}}+e V\right) .
$$

We introduced the Fermi function $f(x)=\left[1+\exp \left(x /\left(k_{\mathrm{B}} T\right)\right)\right]^{-1}$, so that $f\left(E_{\mu}^{\mathrm{S}}-\right.$ $\left.E_{\mathrm{F}}^{\mathrm{S}}\right)$ and $\left[1-f\left(E_{v}^{\mathrm{T}}-E_{\mathrm{F}}^{\mathrm{T}}\right)\right]$ take care of the occupancy of the sample states as well as the density of the unoccupied states of the tip. $E_{\mathrm{F}}^{\mathrm{S}}$ and $E_{\mathrm{F}}^{\mathrm{T}}$ are the Fermi levels of the sample and the tip, respectively. Moreover, a factor 2 has been included to include both spin directions and the energy $-e V$ was added to include a bias voltage $V$ between sample and tip which is usually present during 
STM operation. A similar expression is obtained for the tunneling current from the tip to the sample,

$$
I_{\mathrm{T} \rightarrow \mathrm{S}}=\frac{4 \pi e}{\hbar} \sum_{\mu \nu} f\left(E_{v}^{\mathrm{T}}-E_{\mathrm{F}}^{\mathrm{T}}\right)\left[1-f\left(E_{\mu}^{\mathrm{S}}-E_{\mathrm{F}}^{\mathrm{S}}\right)\right]\left|M_{\mu \nu}\right|^{2} \delta\left(E_{v}^{\mathrm{T}}-E_{\mu}^{\mathrm{S}}+e V\right) .
$$

The total current thus reads

$$
\begin{aligned}
I & =I_{\mathrm{S} \rightarrow \mathrm{T}}-I_{\mathrm{T} \rightarrow \mathrm{S}} \\
& =\frac{4 \pi e}{\hbar} \sum_{\mu \nu}\left[f\left(E_{\mu}^{\mathrm{S}}-E_{\mathrm{F}}^{\mathrm{S}}\right)-f\left(E_{v}^{\mathrm{T}}-E_{\mathrm{F}}^{\mathrm{T}}\right)\right]\left|M_{\mu \nu}\right|^{2} \delta\left(E_{v}^{\mathrm{T}}-E_{\mu}^{\mathrm{S}}+e V\right) .
\end{aligned}
$$

It is useful to separate the energies $E_{\mu}^{\mathrm{S}}$ and $E_{v}^{\mathrm{T}}$ by introducing a second $\delta$ distribution,

$$
\begin{aligned}
I=\frac{4 \pi e}{\hbar} \int \mathrm{d} \varepsilon \sum_{\mu \nu}[ & \left.f\left(E_{\mu}^{\mathrm{S}}-E_{\mathrm{F}}^{\mathrm{S}}\right)-f\left(E_{\nu}^{\mathrm{T}}-E_{\mathrm{F}}^{\mathrm{T}}\right)\right]\left|M_{\mu \nu}\right|^{2} \times \\
& \times \delta\left(E_{v}^{\mathrm{T}}-E_{\mathrm{F}}^{\mathrm{T}}-\varepsilon\right) \delta\left(E_{\mu}^{\mathrm{S}}-E_{\mathrm{F}}^{\mathrm{T}}-e V-\varepsilon\right) .
\end{aligned}
$$

The bias voltage is now modified to compensate a substitution of the tip Fermi energy to the sample Fermi energy in the second $\delta$ distribution: $E_{\mathrm{F}}^{\mathrm{T}}+e V=$ $E_{\mathrm{F}}^{\mathrm{S}}+e \tilde{V}$. This leads to

$$
\begin{aligned}
I=\frac{4 \pi e}{\hbar} \int \mathrm{d} \varepsilon \sum_{\mu \nu}[ & f(\varepsilon+e \tilde{V})-f(\varepsilon)]\left|M_{\mu \nu}\right|^{2} \times \\
& \times \delta\left(E_{v}^{\mathrm{T}}-E_{\mathrm{F}}^{\mathrm{T}}-\varepsilon\right) \delta\left(E_{\mu}^{\mathrm{S}}-E_{\mathrm{F}}^{\mathrm{S}}-e \tilde{V}-\varepsilon\right) .
\end{aligned}
$$

Next, we assume that our system is near zero temperature so that the Fermi functions are eliminated by adjusting the integral boundaries:

$$
I \stackrel{T \rightarrow 0}{=} \frac{4 \pi e}{\hbar} \int_{0}^{-e \tilde{V}} \mathrm{~d} \varepsilon \sum_{\mu \nu}\left|M_{\mu \nu}\right|^{2} \delta\left(E_{\nu}^{\mathrm{T}}-E_{\mathrm{F}}^{\mathrm{T}}-\varepsilon\right) \delta\left(E_{\mu}^{\mathrm{S}}-E_{\mathrm{F}}^{\mathrm{S}}-e \tilde{V}-\varepsilon\right) .
$$

To proceed further, we need to examine the tunnel matrix element $M_{\mu \nu}$.

\subsubsection{The tunnel matrix}

The matrix element $M_{\mu \nu}$, which we defined in eq. (3.225), is evaluated as follows. First, we switch to the real space representation and exploit that the tip potential $\hat{V}_{\mathrm{T}}$ is non-zero in the tip region $\Omega_{\mathrm{T}}$ only,

$$
\begin{aligned}
M_{\mu \nu} & =\left\langle\psi_{v}^{\mathrm{T}}\left|\hat{V}_{\mathrm{T}}\right| \psi_{\mu}^{\mathrm{S}}\right\rangle \\
& =\int_{\Omega_{\mathrm{T}}} \psi_{\mu}^{\mathrm{S}}(\vec{r}) V_{\mathrm{T}}(\vec{r}) \psi_{\nu}^{\mathrm{T} *}(\vec{r}) \mathrm{d} \vec{r} .
\end{aligned}
$$


With the Schrödinger equation for the tip, eq. (3.212), the expression becomes

$$
M_{\mu \nu}=\int_{\Omega_{\mathrm{T}}}\left[\psi_{\mu}^{\mathrm{S}}(\vec{r}) \frac{\hbar^{2}}{2 m} \nabla^{2} \psi_{\nu}^{\mathrm{T} *}(\vec{r})+\psi_{\nu}^{\mathrm{T} *}(\vec{r}) E_{\nu}^{\mathrm{T}} \psi_{\mu}^{\mathrm{S}}(\vec{r})\right] \mathrm{d} \vec{r} .
$$

In the case of elastic scattering, $E_{\mu}^{\mathrm{S}}=E_{\nu}^{\mathrm{T}}$, which is enforced by the $\delta$-distribution in the transition amplitude (3.228), the Schrödinger equation of the sample, eq. (3.211), can be inserted into the second part of eq. (3.238). Note that the integration volume is far away from the sample, so that $V_{\mathrm{S}}(\vec{r})=0, \vec{r} \in \Omega_{\mathrm{T}}$. The matrix element becomes

$$
\begin{aligned}
M_{\mu \nu} & =\frac{\hbar^{2}}{2 m} \int_{\Omega_{\mathrm{T}}}\left[\psi_{\mu}^{\mathrm{S}}(\vec{r}) \nabla^{2} \psi_{\nu}^{\mathrm{T} *}(\vec{r})-\psi_{\nu}^{\mathrm{T} *}(\vec{r}) \nabla^{2} \psi_{\mu}^{\mathrm{S}}(\vec{r})\right] \mathrm{d} \vec{r} \\
& =\frac{\hbar^{2}}{2 m} \int_{\Sigma}\left[\psi_{\mu}^{\mathrm{S}}(\vec{r}) \nabla \psi_{\nu}^{\mathrm{T} *}(\vec{r})-\psi_{\nu}^{\mathrm{T} *}(\vec{r}) \nabla \psi_{\mu}^{\mathrm{S}}(\vec{r})\right] \cdot \mathrm{d} \vec{\sigma}
\end{aligned}
$$

where in the last step Gauss's theorem is applied to convert the volume integral to an integral over the surface $\Sigma$, separating the sample and tip regions.

With tunnel matrix elements of the form (3.239), the expression for the tunneling current (3.235) will be rather difficult to evaluate in practice, since it depends on the wave functions of the tip and sample states. At this point enters the Tersoff-Hamann approximation, which relies on a simplification of the tip wave functions.

\subsubsection{The Tersoff-Hamann approximation}

In scanning tunneling microscopy, the tip is usually thought of as a cluster of atoms with one atom at the apex. The tunneling processes are strongest from the sample to the tip's apex atom, and assumed to be negligible for other atoms of the tip. This is because the distance between the surface and the tip is on atomic scale itself, and thus tunneling to the tip other than the apex atom is strongly suppressed by the exponential decay of the sample wave function. If the apex atom is the only atom of the tip to be considered, the tip can be modeled as a locally spherical potential well [126, 127]. A simple way of how this can be used for an approximation of the tip wave functions was shown by Chen $[124,129]$ and is presented in the following.

At the separation surface $\Sigma$ of the sample and tip regions the sample wave function as well as the tip wave function obey the vacuum Schrödinger equation

$$
\left(-\frac{\hbar^{2}}{2 m} \nabla^{2}+V_{\text {vac }}-E\right) \psi(\vec{r})=0
$$

where $V_{\text {vac }}$ is the vacuum potential and $E$ is the energy associated with the wave function $\psi$. Since tunneling occurs for states close to the Fermi level, i.e. $E \approx E_{\mathrm{F}}$, we can employ the work function $\phi=V_{\text {vac }}-E$ and write

$$
\left(\nabla^{2}-\kappa^{2}\right) \psi(\vec{r})=0
$$


where $\kappa=\sqrt{2 m \phi} / \hbar$. Inside the tip region, the potential of the tip is spherical around the tip apex at position $\vec{r}_{\mathrm{T}}$. The tip wave function can be written as a linear combination of the form

$$
\psi^{\mathrm{T}}(\vec{r})=\sum_{l m} c_{l m} R_{l}\left(\kappa\left|\vec{r}-\vec{r}_{\mathrm{T}}\right|\right) Y_{l m}(\vartheta, \varphi),
$$

where $c_{l m}$ are the expansion coefficients for a product of a radial function $R_{l}\left(\kappa\left|\vec{r}-\vec{r}_{\mathrm{T}}\right|\right)$ and a spherical harmonic $Y_{l m}(\vartheta, \varphi)$. The radial part $R_{l}\left(\kappa\left|\vec{r}-\vec{r}_{\mathrm{T}}\right|\right)$ of the tip wave function is a solution of the modified spherical Bessel differential equation

$$
\frac{\mathrm{d}}{\mathrm{d} x}\left(x^{2} \frac{\mathrm{d} R_{l}(x)}{\mathrm{d} x}\right)-\left(x^{2}+l(l+1)\right) R_{l}(x)=0,
$$

where $x=\kappa\left|\vec{r}-\vec{r}_{\mathrm{T}}\right|$ and $l$ is the quantum number of angular momentum. Furthermore, because the tip wave function has to decay at large distance from the tip, the solution is a modified spherical Bessel function of the second kind,

$$
k_{l}(x)=(-1)^{l} x^{l}\left(\frac{\mathrm{d}}{x \mathrm{~d} x}\right)^{l} \frac{e^{-x}}{x} .
$$

Tersoff and Hamann chose to approximate the tip wave function by an $s$ wave function [126, 127], i. e. all coefficients in the expansion (3.243) except for $c_{00}$ are set to zero. The radial part of the $s$ wave function is the zeroth modified spherical Bessel function of the second kind,

$$
k_{0}\left(\kappa\left|\vec{r}-\vec{r}_{\mathrm{T}}\right|\right)=\frac{e^{-\kappa\left|\vec{r}-\vec{r}_{\mathrm{T}}\right|}}{\kappa\left|\vec{r}-\vec{r}_{\mathrm{T}}\right|},
$$

and the angular dependence of the $s$ wave function is a constant $Y_{00}(\vartheta, \varphi)=$ $1 / \sqrt{4 \pi}=$ const. Hence, the tip wave function is approximated as

$$
\psi^{\mathrm{T}}(\vec{r})=C k_{0}\left(\kappa\left|\vec{r}-\vec{r}_{\mathrm{T}}\right|\right),
$$

where $C$ is a constant.

It is convenient to proceed with the use of the Green's function of the operator $\left(\nabla^{2}-\kappa^{2}\right)$, which is defined via

$$
\left(\nabla^{2}-\kappa^{2}\right) G\left(\vec{r}-\vec{r}_{\mathrm{T}}\right)=-\delta\left(\vec{r}-\vec{r}_{\mathrm{T}}\right)
$$

It reads

$$
G\left(\vec{r}-\vec{r}_{\mathrm{T}}\right)=\frac{\kappa}{4 \pi} k_{0}\left(\kappa\left|\vec{r}-\vec{r}_{\mathrm{T}}\right|\right),
$$

so that we can write the tip wave function in terms of the Green's function 
positive sample bias voltage $V$

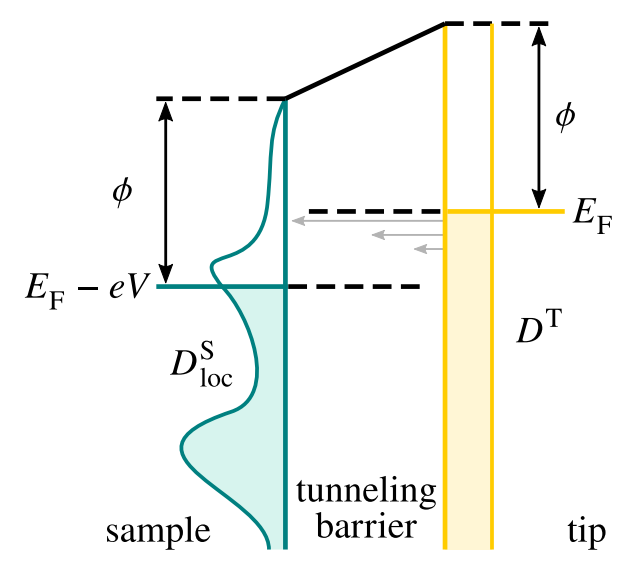

negative sample bias voltage $V$

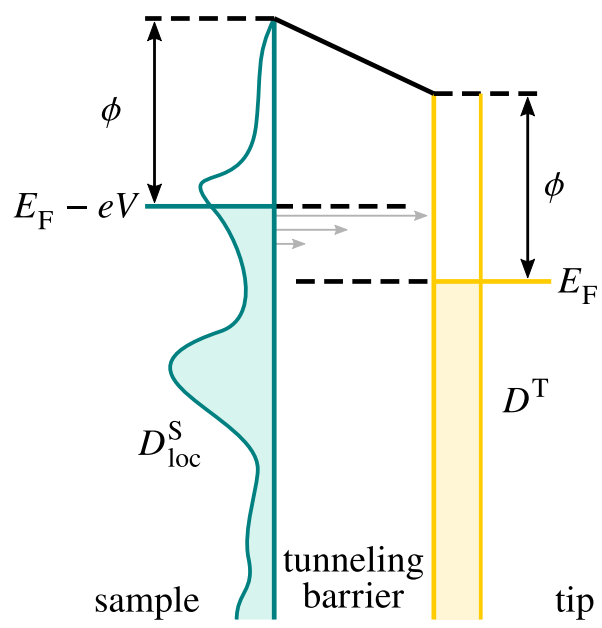

Figure 3.2: Energy level diagram of the tunneling contact in the TersoffHamann approximation at positive (left) and negative (right) sample bias voltage. $D_{\text {loc }}^{\mathrm{S}}$ denotes the local density of states of the sample at the tip apex and $D^{\mathrm{T}}$ is the constant DOS of the tip. Filled areas below $D_{\text {loc }}^{\mathrm{S}}$ and $D^{\mathrm{T}}$ correspond to occupied states. In the Tersoff-Hamann approximation, the work functions of tip and sample coincide, $\phi^{\mathrm{S}}=\phi^{\mathrm{T}} \equiv \phi$. The tunneling probability (gray arrows) is generally higher for higher energies (not accounted for in the Tersoff-Hamann approximation).

(3.249),

$$
\psi^{\mathrm{T}}(\vec{r})=C k_{0}\left(\kappa\left|\vec{r}-\vec{r}_{\mathrm{T}}\right|\right)=\frac{4 \pi C}{\kappa} G\left(\vec{r}-\vec{r}_{\mathrm{T}}\right)
$$

Inserting this result into eq. (3.239) and exploiting the vacuum Schrödinger equation (3.242) for the sample state, one finds the tunnel matrix element for an $s$ wave tip state,

$$
M_{s}=-\frac{2 \pi \hbar^{2} C}{m \kappa} \psi_{\mu}^{\mathrm{S}}\left(\vec{r}_{\mathrm{T}}\right)
$$

In the Tersoff-Hamann approximation, the tunnel matrix element is thus proportional to the sample wave function at the position of the tip.

With the tunnel matrix element (3.251) the tunnel current (3.235) becomes

$$
I \propto \int_{0}^{-e V} \mathrm{~d} \varepsilon[\underbrace{\left[\sum_{v} \delta\left(E_{v}^{\mathrm{T}}-E_{\mathrm{F}}-\varepsilon\right)\right]}_{D^{T}\left(E_{\mathrm{F}}+\varepsilon\right)} \underbrace{\left[\sum_{\mu}\left|\psi_{\mu}^{\mathrm{S}}\left(\vec{r}_{\mathrm{T}}\right)\right|^{2} \delta\left(E_{\mu}^{\mathrm{S}}-E_{\mathrm{F}}-e V-\varepsilon\right)\right]}_{D_{\mathrm{loc}}^{\mathrm{S}}\left(E_{\mathrm{F}}+e V+\varepsilon, \vec{r}_{\mathrm{T}}\right)},
$$

where $D^{\mathrm{T}}$ is the density of states (DOS) of the tip and $D_{\text {loc }}^{\mathrm{S}}$ is the local density of states (LDOS) of the sample. Note that in the approximation it was assumed 
that the Fermi levels of tip and sample coincide, $E_{\mathrm{F}}^{\mathrm{S}}=E_{\mathrm{F}}^{\mathrm{T}} \equiv E_{\mathrm{F}}$, so that $\tilde{V}=V$. Moreover, because of the simplification of the tip, in the TersoffHamann approximation the tip DOS becomes a constant, so that the tunneling current simplifies to

$$
I \propto \int_{0}^{-e V} \mathrm{~d} \varepsilon D_{\mathrm{loc}}^{\mathrm{S}}\left(E_{\mathrm{F}}+e V+\varepsilon, \vec{r}_{\mathrm{T}}\right) .
$$

Hence, the tunneling current is proportional to the integrated LDOS of the sample at the position of the tip apex $\vec{r}_{\mathrm{T}}$.

Figure 3.2 shows a schematic energy diagram of the STM tunneling contact in the Tersoff-Hamann approximation for a positive and a negative bias voltage, respectively. With a positive bias voltage the sample states are shifted to lower energies and a tunneling current from the tip into the empty sample states is formed. Conversely, in the case of a negative bias voltage the sample states are shifted upwards in energy and tunneling occurs from the occupied sample states into the unoccupied tip states. Note that the Tersoff-Hamann approximation neglects an energy dependence of the tunneling matrix element, as it assumes a small bias voltage, so that the tunneling particles are roughly at the Fermi level. Nevertheless, in fig. 3.2 we illustrate the energy dependence of the tunneling probability (3.209) via the length of the gray arrows, i.e. the contribution to the tunneling current should be increased for higher electron energies.

\subsubsection{Scanning tunneling spectroscopy}

The derivative of eq. (3.253) with respect to the bias voltage is proportional to the sample LDOS at $\vec{r}_{\mathrm{T}}$,

$$
\frac{\mathrm{d} I}{\mathrm{~d} V} \propto D_{\mathrm{loc}}^{\mathrm{S}}\left(E_{\mathrm{F}}+e V, \vec{r}_{\mathrm{T}}\right)
$$

Equation (3.254) illustrates that by measuring the dependence of the tunneling current on the sample bias voltage, information about the sample LDOS can be acquired.

Experimentally, $I(V)$ curves for a sample surface area can be recorded quasi-simultaneously to a constant current topography. For the measurement of a constant current topography the tip scans the sample surface by visiting a discrete set of measurement points. To keep the tunneling current constant, the tip height is adjusted by the feedback loop of the scanning tunneling microscope when moving from one measurement point to the next measurement location. When a measurement for spatially resolved scanning tunneling spectroscopy data is performed quasi-simultaneously to the measurement of the constant current topography, at each measurement point, after the tip height has been adjusted, the feedback loop is temporarily deactivated and the tip position remains fixed. Then an $I(V)$ curve is obtained by recording the tunneling current while tuning the bias voltage. When the measurement of the $I(V)$ curve is complete, the bias voltage is reset to the initial value, the feedback 
loop for the tip height adjustment is reactivated and the tip continues to the next measurement location, where the process is repeated. For each of the $I(V)$ curves, the differential conductance (3.254) is computed from the changes in the $I(V)$ data. Due to the relation of the differential tunneling conductance to the electronic spectrum of the sample, this experimental approach is commonly referred to as scanning tunneling spectroscopy (STS).

Although eq. (3.254) establishes a link between the experimentally accessible $I(V)$ curve and the theoretically obtainable sample LDOS, it has to be emphasized that a few rather crude approximations were made during the derivation. Hence, theoretical predictions for STS measurements remain at a qualitative level. 


\section{DFT bulk simulations of rare-earth hex- aborides}

In this chapter results of bulk simulations of the light rare-earth hexaborides, based on density functional theory (DFT), are presented. First we give an overview of the electronic structure of the boron network within a rare-earth hexaboride crystal. For this purpose, in section 4.2 we show the density of states (DOS) from DFT simulations of the $\left[\mathrm{B}_{6}\right]^{2-}$ crystal network in a homogeneous positive charge background and project the DOS onto tight binding orbitals of the boron octahedron complex, which are defined in section 4.1. Subsequently, in section 4.3 we present the results of DFT bulk simulations on $\mathrm{LaB}_{6}$ and compare the outcome to the $\left[\mathrm{B}_{6}\right]^{2-}$ results. Furthermore, we compare the DOSs from simulations of $\mathrm{LaB}_{6}$ with the Perdew-Burke-Ernzerhof (PBE) functional and with the PBE0r hybrid functional. In $\mathrm{LaB}_{6}$ the effect of the hybrid functional exchange mixing is shown to be rather small because of the absence of $4 f$ electrons. This changes for other light rare-earth hexaborides with occupied $4 f$ orbitals. We demonstrate the effect of a mixing of exact local exchange with the PBE functional for $\mathrm{CeB}_{6}, \mathrm{PrB}_{6}$, and $\mathrm{NdB}_{6}$ in section 4.4. There, we compare the DOSs that are obtained with the PBE functional and the PBE0r hybrid functional with different exchange mixing factors. In section 4.5 we discuss the results of this chapter, which motivate a model calculation that is the topic of chapter 5 .

\subsection{The electronic structure of a $B_{6}$ octahedron}

For our analysis of the electronic structure of the boron network we exploit the fact that the eigenstates of the Hamiltonian reflect the symmetries of the underlying system. The Hamiltonian can be simplified and brought to a blockdiagonal shape by choosing a basis of symmetrized states, which are eigenstates to the symmetry operators of the system at hand. For the $\mathrm{B}_{6}$ complex we have an octahedral symmetry. The symmetrized states are formed from linear combinations of the atomic orbitals of the individual boron atoms. In the following we briefly summarize the theoretical foundation on how the eigenstates of a symmetry operator of the system lead to a blockdiagonal shape of the Hamiltonian matrix $[130,131]$. Subsequently, we describe our choice of symmetrized tight-binding orbitals for the $\mathrm{B}_{6}$ octahedra. 


\subsubsection{Exploiting symmetries to diagonalize the Hamiltonian}

Let $\hat{S}$ be a unitary operator that transforms the states of a basis $\{|\Psi\rangle\}$ into the states of a basis $\left\{\left|\Psi^{\prime}\right\rangle\right\}$, i. e. for the $i$-th state of each basis, $\left|\Psi_{i}^{\prime}\right\rangle=\hat{S}\left|\Psi_{i}\right\rangle$ and $\left|\Psi_{i}\right\rangle=\hat{S}^{\dagger}\left|\Psi_{i}^{\prime}\right\rangle . \hat{S}$ is a symmetry operator, if the Hamiltonian is invariant under the transformation $\hat{S}$ so that $\left|\Psi_{i}\right\rangle$ and $\left|\Psi_{i}^{\prime}\right\rangle$ are solutions to the same Schrödinger equation (see e. g. [130, 131]). I. e.

$$
\hat{H} \hat{S}^{\dagger}\left|\Psi_{i}^{\prime}\right\rangle=\hat{H}\left|\Psi_{i}\right\rangle=E_{i}\left|\Psi_{i}\right\rangle=E_{i} \hat{S}^{\dagger}\left|\Psi_{i}^{\prime}\right\rangle \Leftrightarrow \hat{S} \hat{H} \hat{S}^{\dagger}\left|\Psi_{i}^{\prime}\right\rangle=E_{i}\left|\Psi_{i}^{\prime}\right\rangle,
$$

and the Schrödinger equations for $\left|\Psi_{i}\right\rangle$ and $\left|\Psi_{i}^{\prime}\right\rangle$ are the same, if

$$
\hat{S} \hat{H} \hat{S}^{\dagger}=\hat{H},
$$

which is equivalent to the commutator relation

$$
[\hat{H}, \hat{S}]=0 \text {. }
$$

For a normalized eigenstate $\left|\Phi_{i}\right\rangle$ of $\hat{S}$ with eigenvalue $s_{i}$, we find

$$
\begin{gathered}
1=\left\langle\Phi_{i} \mid \Phi_{i}\right\rangle=\left\langle\Phi_{i}\left|\hat{S}^{\dagger} \hat{S}\right| \Phi_{i}\right\rangle= \\
\left.\Leftrightarrow \Phi_{i}\left|\hat{S}^{\dagger}\right| \Phi_{i}\right\rangle s_{i}=\left\langle\Phi_{i} \mid \Phi_{i}\right\rangle s_{i}^{*} s_{i}=s_{i}^{*} s_{i}
\end{gathered}
$$

Let $\left|\Phi_{1}\right\rangle$ and $\left|\Phi_{2}\right\rangle$ be eigenstates of the symmetry operator $\hat{S}$ with eigenvalues $s_{1}$ and $s_{2}$, respectively. Then

$$
\begin{aligned}
\left\langle\Phi_{1}|\hat{H}| \Phi_{2}\right\rangle & =\left\langle\Phi_{1}\left|\left(s_{1}^{*}\right)^{-1} \hat{S}^{\dagger} \hat{H} \hat{S} s_{2}^{-1}\right| \Phi_{2}\right\rangle=\left\langle\Phi_{1}\left|\hat{S}^{\dagger} \hat{S} \hat{H}\right| \Phi_{2}\right\rangle\left(s_{1}^{*}\right)^{-1} s_{2}^{-1} \\
& =\left\langle\Phi_{1}|\hat{H}| \Phi_{2}\right\rangle\left(s_{1}^{*}\right)^{-1} s_{2}^{-1}=\left\langle\Phi_{1}|\hat{H}| \Phi_{2}\right\rangle s_{1} s_{2}^{-1}
\end{aligned}
$$

where in the second equality the commutator (4.3) was exploited. Furthermore, eq. (4.5) was inserted in the last equality. Multiplying the equation by $s_{2}$ and subtracting the expression on the left side leads to

$$
\left\langle\Phi_{1}|\hat{H}| \Phi_{2}\right\rangle\left(s_{1}-s_{2}\right)=0
$$

Hence, if the eigenvalues differ, $s_{1} \neq s_{2}$, the matrix element $\left\langle\Phi_{1}|\hat{H}| \Phi_{2}\right\rangle$ is equal to zero. In the basis of the eigenstates of the symmetry operator $\hat{S}$, in which the basis states are ordered according to their eigenvalues with respect to $\hat{S}$, the Hamiltonian matrix then is blockdiagonal. This means that by choosing basis states that reflect the symmetry of the system at hand, the diagonalization of the full Hamiltonian can be simplified to diagonalizations of smaller subblocks of the Hamiltonian matrix. 


\subsubsection{Symmetrized orbitals of the $B_{6}$ octahedra}

Following section 4.1.1, the Hamiltonian describing a boron octahedron can be brought into a blockdiagonal form by selecting basis states that, up to a phase factor, are invariant with respect to the symmetry transformations of the octahedral geometry. Among the most obvious symmetry transformations of an octahedron are reflections at the three mirror planes that contain two opposite corners of the octahedron for two spatial directions each. We select basis states, which are formed as linear combinations of the $2 s$ and $2 p$ orbitals of the individual boron atoms and and which are symmetric or antisymmetric with respect to reflections at the three mirror planes introduced above [132]. They are obtained by constructing a prototype set of tight-binding orbitals, in which each tight-binding orbital is formed as a linear combination of two atomic orbitals of the same type, contributed by the two atoms at two opposite corners of the octahedron. The prototypical orbitals are sorted according to their eigenvalue $(+1,-1)$ with respect to the symmetry operator that represents the reflection at one of the three mirror planes. This process is repeated for each of the two resulting blocks for the other two reflection planes. Subsequently, tight-binding orbitals of higher symmetry are formed by further linear combinations of the prototypical orbitals within each of the resulting blocks, e. g. by considering rotational symmetries.

Since a boron octahedron comprises six boron atoms, each contributing an $s$, a $p_{x}$, a $p_{y}$, and a $p_{z}$ orbital, a total of 24 tight-binding orbitals is obtained. Figure 4.1 shows schematics of the chosen tight-binding orbitals of the boron complex. Our choice of the tight-binding orbitals mostly matches that of Longuet-Higgins and Roberts [132].

Note, that the Hamiltonian of an isolated boron octahedron differs from the Hamiltonian of a periodic lattice of boron octahedra. Nevertheless, if the octahedron orbitals are sufficiently localized and the overlap with orbitals of neighboring sites is negligible, these orbitals and their periodic images should constitute a suitable basis to keep the Hamiltonian of the periodic lattice approximately block diagonal.

We did not form further linear combinations for the orbitals 1-6 in analogy to their inward-directed pendants, cf. orbitals 7-9, 10-12, and 22-24. It is useful to represent these orbitals as bonds and anti-bonds of two neighboring boron octahedra since they mainly contribute to inter-octahedron bonding and not to the stability of the $\mathrm{B}_{6}$ complex. Schematics of such a bond and anti-bond between two neighboring octahedra are depicted in fig. 4.2.

\section{2 $\mid$ Bulk simulation of the boron network}

We begin our DFT-based theoretical studies of the rare-earth hexaborides with a simulation of the boron network, where the rare-earth site is left empty. Following the discussion of section 2.3, two additional electrons were added to the unit cell. Moreover, the system is embedded into a homogeneous positive charge background, which replaces the metal cations. Hence, in total the sim- 

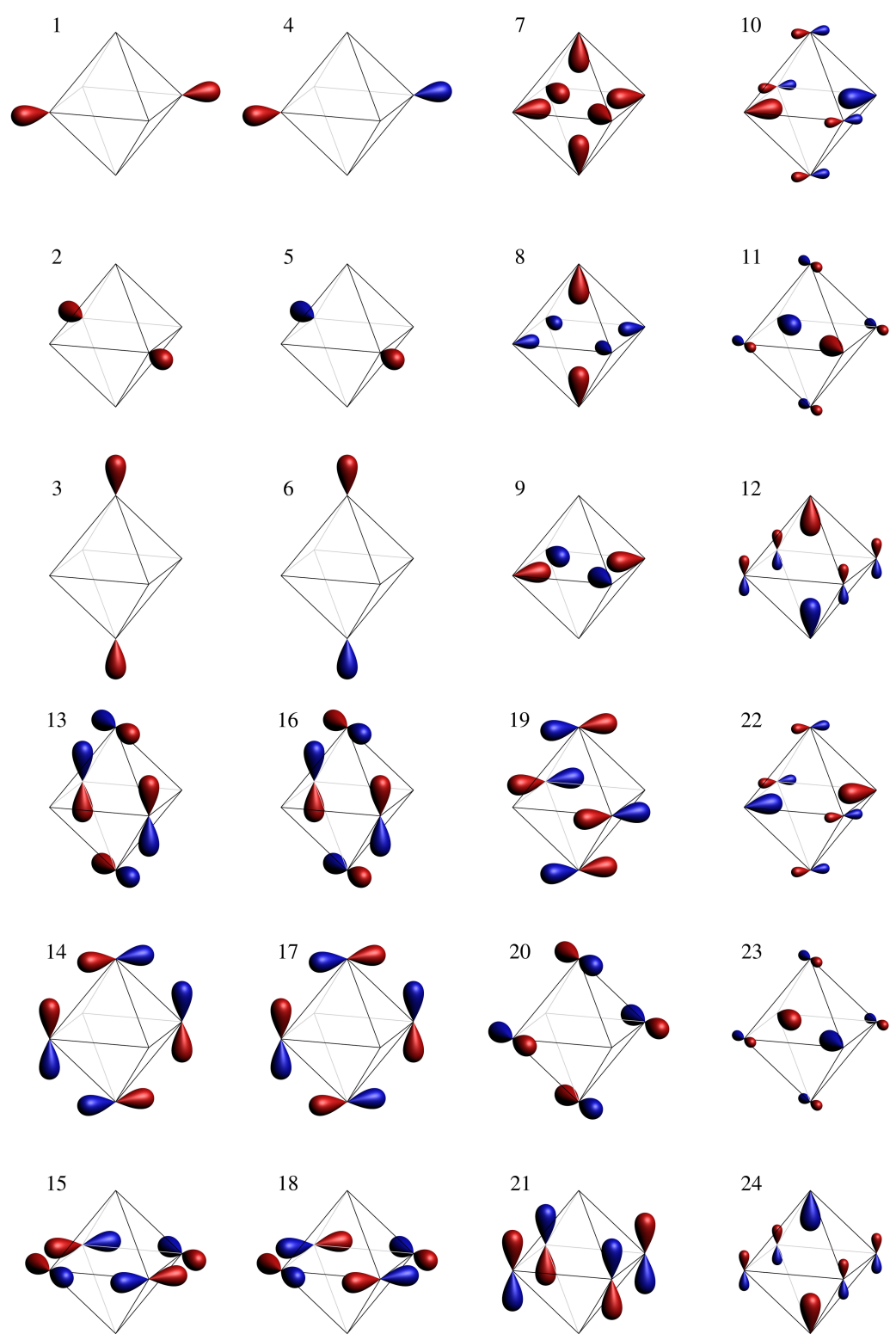

Figure 4.1: Schematics of the tight-binding orbitals of a boron octahedron. Single lobes represent $s p$-hybrid orbitals, double lobes depict $p$ orbitals. Positive and negative phase of the wavefunction is shown in red and blue, respectively.

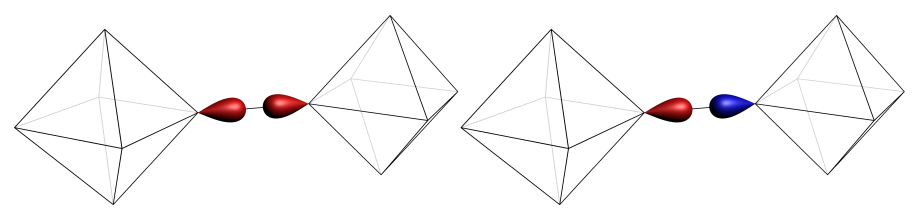

Figure 4.2: Schematics of bonding and anti-bonding orbitals between two neighboring octahedra. 


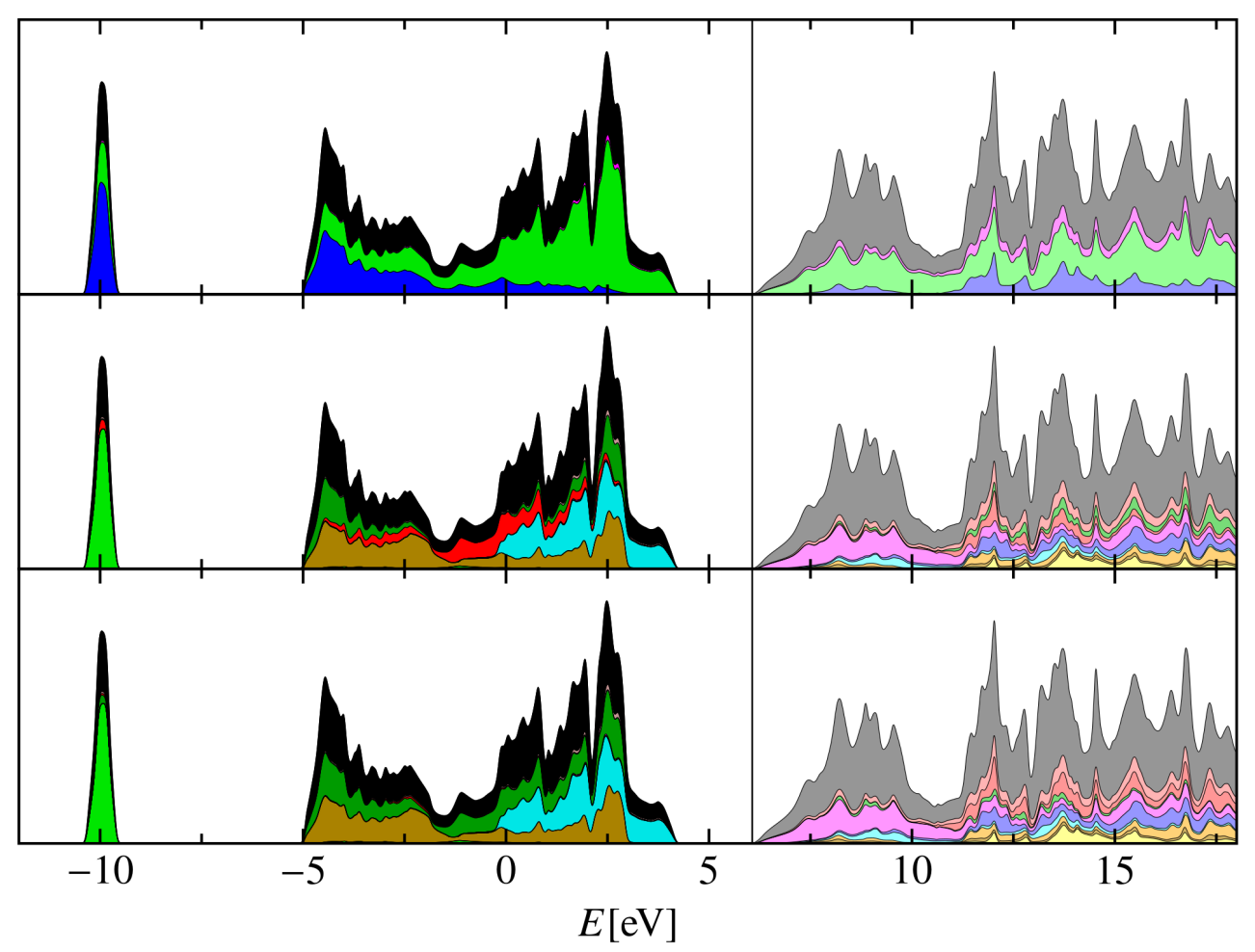

Figure 4.3: Projected density of states of the bare $\left[\mathrm{B}_{6}\right]^{2-}$ framework. The total density of states is depicted in black. Projected DOS contributions are colored and stacked. The lowest unoccupied molecular orbital is marked by the vertical black line at $6.07 \mathrm{eV}$. Top: DOS projected onto boron $s$ orbitals (blue), $p$ orbitals (green), and $d$ orbitals (magenta). Center: DOS projected onto the tight-binding orbitals of the boron octahedron, see fig. 4.1. Color code by orbital numbers: 7 (light green), 10-12 (dark gold), 13-15 (cyan), 4-6 (dark green), 1-3 (red), 19-21 (magenta), 16-18 (blue), 22-24 (orange), 8-9 (yellow), boron $d$ (reddish brown). Bottom: Same as in the center picture, but the tight-binding orbitals 1-6 are replaced by inter-octahedron bonding (dark green) and anti-bonding orbitals (red, unoccupied only), see fig. 4.2 .

ulation unit cell contains six boron atoms in an octahedral arrangement, two additional electrons and a homogeneous charge background. For the simulations, we employed the Perdew-Burke-Ernzerhof (PBE) functional for exchange and correlation. A unit cell spacing of $a_{\mathrm{La}}=4.15 \AA$ was chosen, which is the bulk lattice constant of $\mathrm{LaB}_{6}$ [38-42]. Furthermore, the simulations were performed on a grid of $(9 \times 9 \times 9) \vec{k}$-points. The number of projector functions was set to $(2,2,1)$ for the boron $(s, p, d)$ shells, respectively. We used plane wave cutoffs of $50 \mathrm{Ry}$ for the wave functions and $100 \mathrm{Ry}$ for the charge density.

The projected density of states (PDOS) of the $\left[\mathrm{B}_{6}\right]^{2-}$ skeleton is depicted in fig. 4.3, with the lower edge of the unoccupied states indicated by the vertical black line at $6.07 \mathrm{eV}$. In the upper graph, the density of states is projected onto boron $s$ (blue), $p$ (green), and $d$ (magenta) states. The material is an insulator 
with a gap of $1.8 \mathrm{eV}$. The occupied states are $s$ and $p$ states, showing a peak at $-9.9 \mathrm{eV}$ and a broad band in the energy range from $-5 \mathrm{eV}$ to $4.25 \mathrm{eV}$. There is a general trend of more $s$ states accumulating at lower energies, whereas more $p$ states can be found at higher energies. In the unoccupied states the energy region from $6.07 \mathrm{eV}$ to $11.10 \mathrm{eV}$ is dominated by $p$ states. Above, there is a mixture of $s$ and $p$ states with also $d$-states starting to contribute.

In the center plot of fig. 4.3 we show the projection of the DOS onto the boron tight-binding orbitals of fig. 4.1. The peak at $-9.9 \mathrm{eV}$ is clearly identified to originate from the bonding $a_{1 g}$-type orbital no. 7 . This orbital bonds all boron constituents and significantly contributes to the stability of the octahedral complex, which is reflected in its low energy level. The occupied states in the range from $-5 \mathrm{eV}$ to $4.25 \mathrm{eV}$ are composed of four types of tight-binding orbitals. First, we have the outward directed orbitals 1-3 (red) and 4-6 (dark green). Second, orbitals 10-12 (dark gold) largely contribute, which form bonds between five boron constituents in the pyramidal volumes on each side of the mirror planes defined above. The two sides of the bonding orbital have opposite phases, giving the orbitals a $p$-type appearance. Third, we have the bonding orbitals 13-15 (cyan) which are formed between four boron constituents, each two lying on opposite corners of the octahedron. Each orbital consists of four bonds, where each bond is formed between two neighboring boron constituents along an outer edge of the octahedron. Going around the octahedron, the phase of the four individual bonds alternates and the orbitals have a $d$-type appearance. Because of their structure the orbitals 13-15 are weaker bonding than the orbitals 10-12 and do not stabilize the octahedron as strongly. This is reflected in their generally higher energy.

In the unoccupied states, there are many orbitals of anti-bonding character. The energy range from $6.07 \mathrm{eV}$ to $11.10 \mathrm{eV}$ is dominated by the $t_{2 u}$-type orbitals 19-21. Above $11.10 \mathrm{eV}$, there is variety of orbitals, including the anti-bonding orbitals 8-9 (yellow), 16-18 (blue), and further contributions of orbitals 19-21.

The bottom plot of fig. 4.3 shows the same projection as the middle plot, with the exception, that the outward-pointing orbitals 1-6 are replaced by the inter-octahedron bonding (dark green) and anti-bonding (red) orbitals, which are depicted in fig. 4.2. It is evident, that the PDOS of the inter-octahedron bonds fits the PDOS of the orbitals $1-6$ at energies $-5 \mathrm{eV}$ to $3.10 \mathrm{eV}$. The anti-bonding orbitals occur at higher energies within the unoccupied states.

From the PDOS in fig. 4.3 it is clear that all bonding-type orbitals of the boron octahedra are occupied and all anti-bonding orbitals remain unoccupied. This explains the high stability of the octahedral units. Furthermore, neighboring octahedra are connected by bonds that are in the same energy region as most of the intra-octahedron bonding orbitals. This explains the rigidity of the boron network.

The density of states of our simulations of the boron network agrees with the early work of Longuet-Higgins and Roberts, who predicted the intra-octahedron bonding orbitals and the inter-octahedron boron bonds to comprise the occupied boron states [132]. Furthermore, in accordance with their results, the $t_{2 u}$-type orbitals (19-21 in fig. 4.1) are found in a band slightly above the Fermi level. 


\subsection{DFT bulk simulations of lanthanum hexaboride}

The first and simplest of the rare-earth hexaborides is lanthanum hexaboride $\left(\mathrm{LaB}_{6}\right)$, since lanthanum does not have occupied $f$-electron states in its ground state configuration. We performed DFT bulk simulations of $\mathrm{LaB}_{6}$, firstly as a reference for bulk simulations of other light rare-earth hexaborides and, secondly, as a precursor for a surface study of the $\mathrm{LaB}_{6}(001)$ cleavage plane that is presented in chapter 6 . For the simulations, we employed the generalized gradient approximation in form of the PBE functional, and the hybrid functional PBE0r. The simulations were performed with one $\mathrm{LaB}_{6}$ bulk unit cell and a lattice constant of $a_{\mathrm{La}}=4.15 \AA$ [38-42].

In the rare-earth hexaborides the rare-earth's $5 s$ and $5 p$ orbital shells act as semi-core states. Their energies are well above those of lower lying rare-earth core states and rather close to the rare-earth $5 d$, and $4 f$ levels, as well as to the boron band. In the augmentation within the PAW method we consider the lanthanum $5 s, 5 p, 5 d, 6 s, 6 p$, and $4 f$ orbital shells, as well as the boron $2 s, 2 p$, and $3 d$ orbital shells to be valence states. For the boron $(s, p, d)$ and lanthanum $(s, p, d, f)$ orbitals, respectively, we use a set of $(2,2,1)$ and $(2,2,2,1)$ projector functions. The full setups for boron and lanthanum are given in appendix B. We set the plane wave cutoff for the expansion of the pseudo wave functions to $50 \mathrm{Ry}$ and for the expansion of the pseudo charge density to $100 \mathrm{Ry}$. The simulations are performed on a $(9 \times 9 \times 9) k$-point grid.

\subsubsection{Results with the PBE functional}

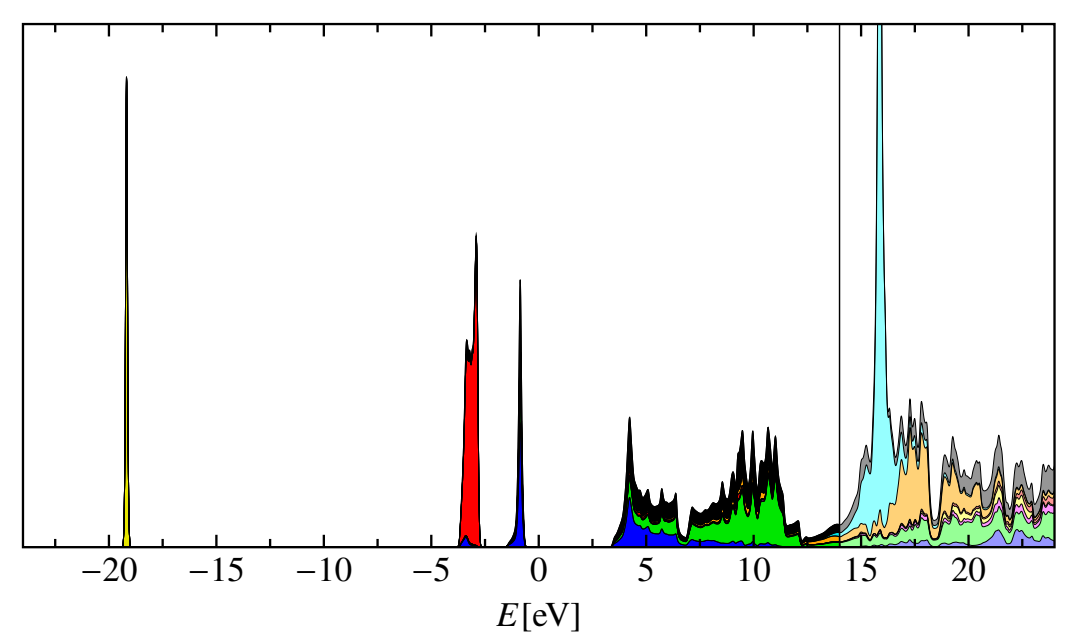

Figure 4.4: Angular momentum-resolved density of states of the DFT bulk simulation of $\mathrm{LaB}_{6}$. The Fermi level is at $14 \mathrm{eV}$. Total DOS in black. Colors: projection of the DOS onto B $s$ (blue), B $p$ (green), $\mathrm{B} d$ (magenta), La $s$ (yellow), La $p$ (red), La $d$ (orange), and La $f$ (cyan) orbitals. The projected quantities are stacked.

A large scale overview of the angular momentum projected DOS of $\mathrm{LaB}_{6}$, simulated with the PBE-functional, is shown in fig. 4.4. $\mathrm{LaB}_{6}$ is shown to be 


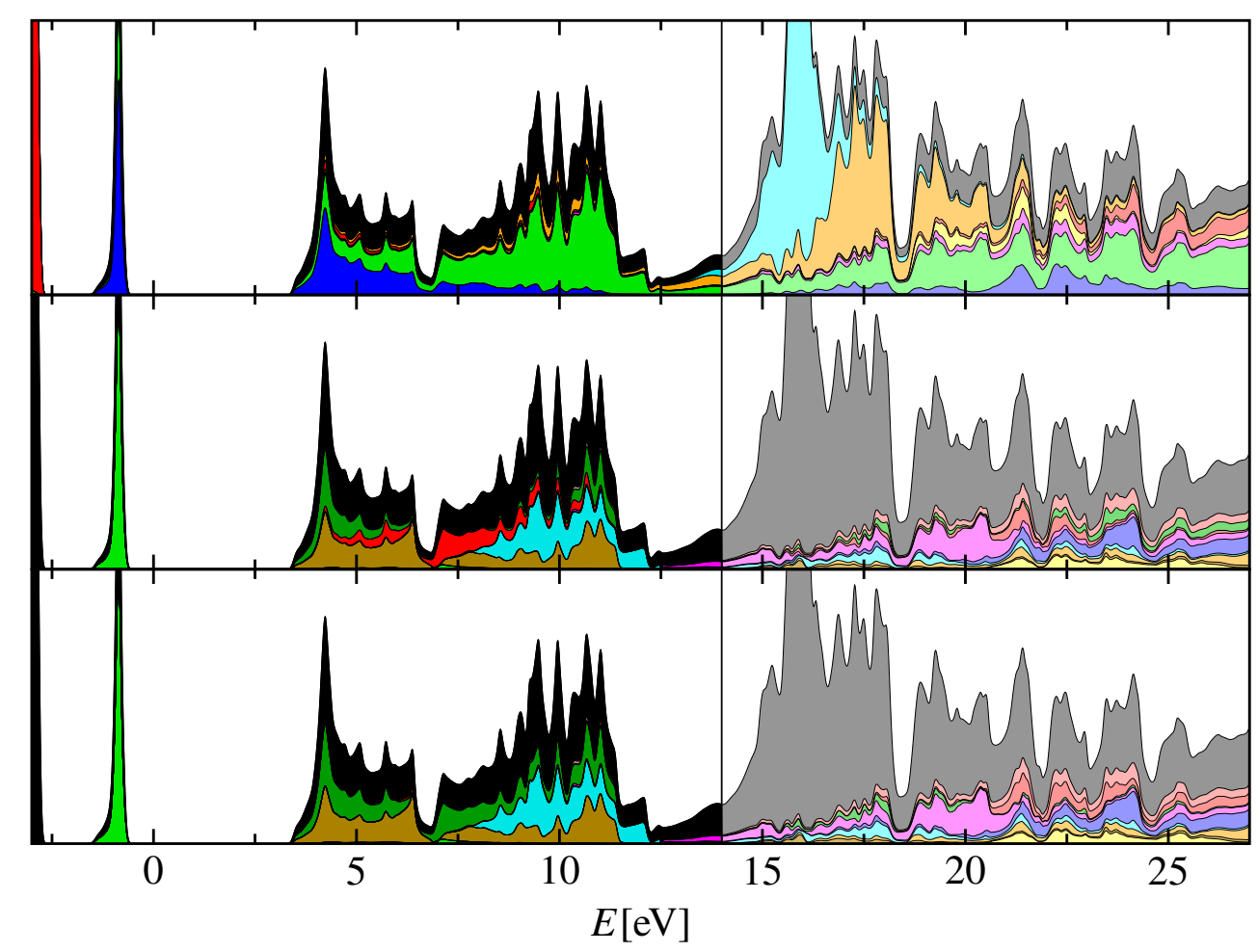

Figure 4.5: Orbital resolved density of states of the DFT bulk simulation of $\mathrm{LaB}_{6}$. Total DOS in black. Top: projection onto B $s$ (blue), B $p$ (green), B $d$ (magenta), La $s$ (yellow), La $p$ (red), La $d$ (orange), and La $f$ (cyan) orbitals. Center: DOS projected onto the tightbinding orbitals of the boron octahedron, see fig. 4.1. Color code by orbital numbers: 7 (light green), 10-12 (dark gold), 13-15 (cyan), 4-6 (dark green), 1-3 (red), 19-21 (magenta), 16-18 (blue), 22-24 (orange), 8-9 (yellow), boron $d$ (reddish brown). Bottom: Same as in the center picture, but the tight-binding orbitals 1-6 are replaced by inter-octahedron bonding (dark green) and anti-bonding orbitals (red), see fig. 4.2.

a metal with the Fermi energy at $E_{\mathrm{F}}=14 \mathrm{eV}$. A zoomed-in version of the projected DOS, which leaves out the low-lying La $s$ states at $-19.1 \mathrm{eV}$ and focuses on the states above the La $p$ states at $-3.2 \mathrm{eV}$, is depicted in the top plot of fig. 4.5. The broad, boron-dominated valence band from $3.4 \mathrm{eV}$ to $12.3 \mathrm{eV}$ and the lower lying boron $s$ peak at $-0.9 \mathrm{eV}$ bear a strong resemblance to the DOS of the occupied states in the $\left[\mathrm{B}_{6}\right]^{2-}$ simulation. In the vicinity of $E_{\mathrm{F}}$, La $d$ states and $\mathrm{B} p$ states are present. Moreover, unoccupied $f$-electron states stretch from $E_{\mathrm{F}}$ to $E_{\mathrm{F}}+4.2 \mathrm{eV}$, with a large peak at $E_{\mathrm{F}}+1.9 \mathrm{eV}$. Above the $\mathrm{La}$ $f$ orbitals, a large contribution from the La $d$ orbitals is found.

For a comparison to the DOS of the $\left[\mathrm{B}_{6}\right]^{2-}$ boron network, the PDOS of the boron tight binding orbitals is depicted in the center and bottom plots of fig. 4.5. Except for the small additional admixture of the La $d$ states, the PDOS in the energy region of $-2.5 \mathrm{eV}$ to $12.3 \mathrm{eV}$ matches the PDOS of the boron skeleton at energies between $-12 \mathrm{eV}$ to $5 \mathrm{eV}$ and is dominated by the $\mathrm{B}_{6}$ bonding orbitals. 


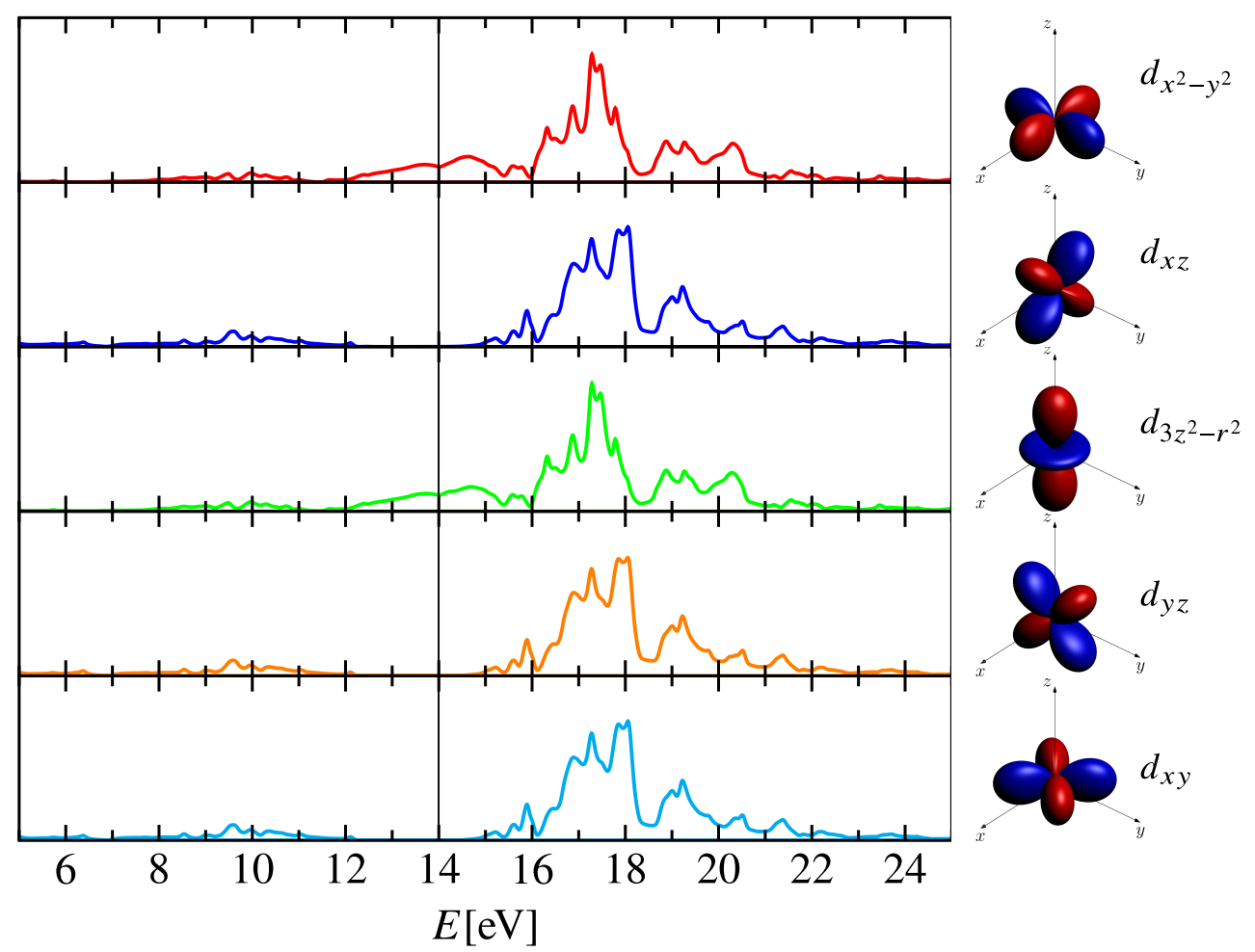

Figure 4.6: PDOS of the La $d$ orbitals. The Fermi level is indicated by the black vertical line at $14 \mathrm{eV}$.

Above this boron valence band no gap is found, which is in contrast to the DOS of the $\left[\mathrm{B}_{6}\right]^{2-}$ boron network and can be explained by the additional electron in the $\mathrm{LaB}_{6}$ unit cell. Between $12.3 \mathrm{eV}$ and $21 \mathrm{eV}$, the PDOS of $\mathrm{LaB}_{6}$ exhibits contributions of various boron tight-binding orbitals as well as of the La $d$ and $f$ states. Here, among the boron tight-binding orbitals, the anti-bonding orbitals 19-21 are predominant, with the exception of the energy range between $15.8 \mathrm{eV}$ and $18.4 \mathrm{eV}$, which is where the La $d$ and La $f$ states dominate the DOS.

From the comparison of the DOS of the $\left[\mathrm{B}_{6}\right]^{2-}$ crystal and the DOS of $\mathrm{LaB}_{6}$ it is evident that the presence of the additional La atom does not alter the PDOS of the occupied bonding boron tight-binding orbitals significantly. However, the anti-bonding tight-binding orbitals 19-21 are spread out further and extend below the Fermi level. Together with the additional share of the La $d$ electrons this renders the material metallic.

In fig. 4.6 the projection of the DOS onto the individual $d$ orbitals of the La ion is shown. Due to the symmetry of the hexaboride crystal, the $d$ orbitals split into $t_{2 g}\left(d_{x y}, d_{x z}, d_{y z}\right)$ and $e_{g}\left(d_{x^{2}-y^{2}}, d_{3 z^{2}-r^{2}}\right)$ orbitals. Notably, in the vicinity of the Fermi level the $e_{g}$ orbitals have a non-zero PDOS, while the PDOS of the $t_{2 g}$ orbitals drops to zero. In this energy range, the Kohn-Sham wave functions show a hybridization of the $e_{g}$ orbitals and the boron orbitals 19-21, which explains the deformation of the boron states in comparison with the bare $\left[\mathrm{B}_{6}\right]^{2-}$ system. 


\subsubsection{Results with the PBEOr hybrid functional}

LSDA and GGA functionals have deficits in the treatment of strongly correlated electron systems (c.f. section 3.9.4), which most notably include an incomplete compensation of the self-interaction. For this reason an approach going beyond the GGA is necessary for the simulations of rare-earth hexaborides that have a partly occupied $4 f$ shell. In this thesis, we apply the PBE0r hybrid functional (c.f. section 3.9.6) and examine its impact on the DFT results. Although the strongly localized $4 f$ states are unoccupied in the case of $\mathrm{LaB}_{6}$, the PBE0r functional was used on $\mathrm{LaB}_{6}$ to establish a reference point for simulations of other rare-earth hexaborides.

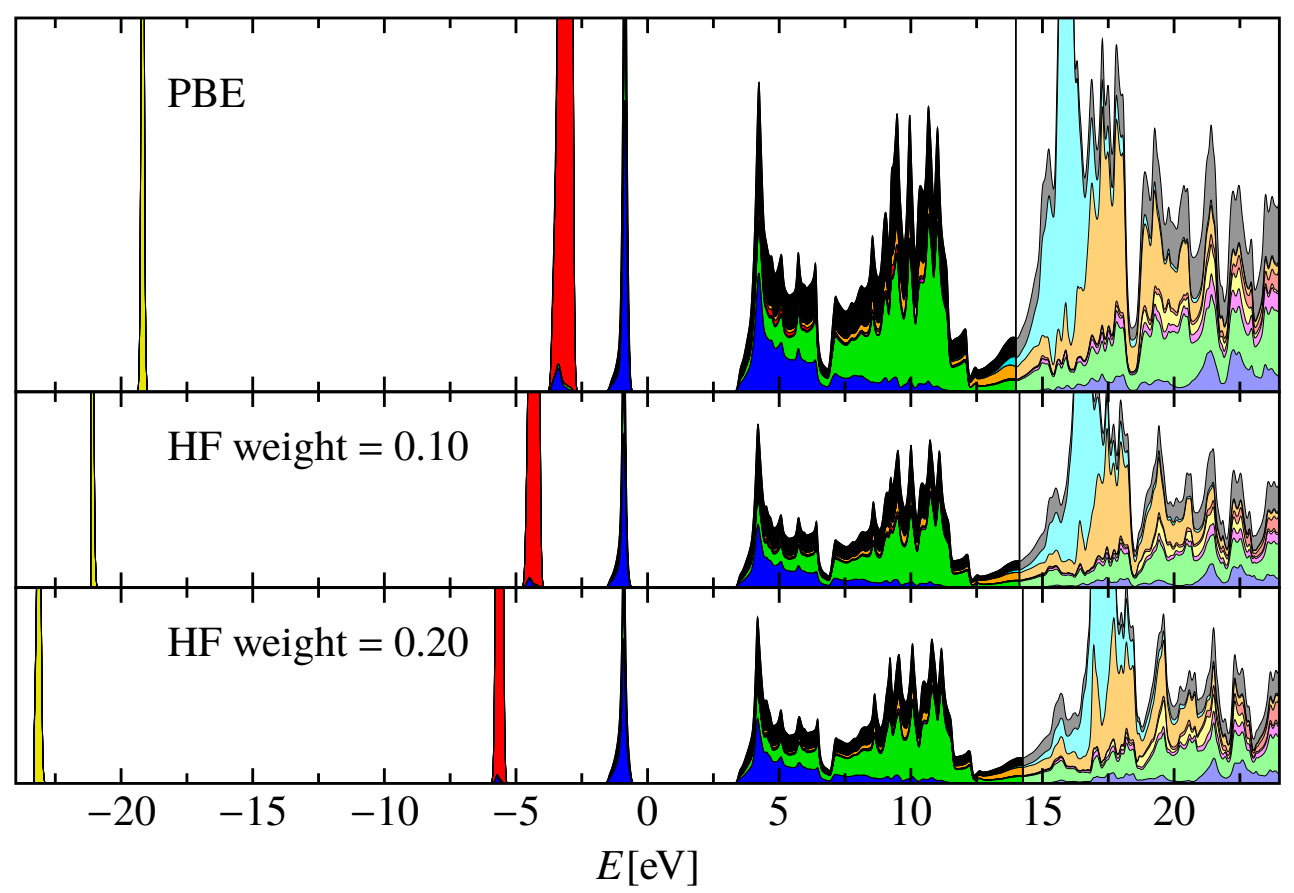

Figure 4.7: Comparison of the DOS of $\mathrm{LaB}_{6}$ from a PBE simulation and simulations made with the PBE0r functional with different mixing factors for the Hartree-Fock exchange. Color code: B $s$ blue, B $p$ green, B $d$ magenta, La $s$ yellow, La $p$ red, La $d$ orange, La $f$ cyan.

A comparison of the DOS of simulations of $\mathrm{LaB}_{6}$ with the PBE functional and the PBE0r functional with different exchange mixing factors $\eta$ (also labeled HF weight) is presented in fig. 4.7. The respective mixing factors were used for lanthanum as well as for the boron atoms. For $\mathrm{LaB}_{6}$, where the $f$ orbitals are unoccupied, the effect of the PBE0r hybrid functional is rather small. The use of the hybrid functional leads to noticeable shifts of the occupied La $s$ and $p$ states to lower energies, whereas the $f$ states are shifted to larger energies. For larger hybrid functional mixing factors the shift length increases. The impact on the La $d$ electrons is small and the boron orbitals are unaffected. 


\subsubsection{Discussion}

The angular-momentum projected DOS of our simulation of $\mathrm{LaB}_{6}$ with the PBE functional is in good agreement with previous data of GGA simulations by Uijttewaal et al. [52] and with results of LSDA $+U$ calculations by Singh et al. [133]. Specifically, the unoccupied $f$-state peak is found at approximately $E_{\mathrm{F}}+$ $2 \mathrm{eV}$. In contrast, in a DOS derived from augmented plane wave bandstructure calculations in the local density approximation by Harima et al. [134], which was presented in the paper of Kimura et al. [135], the unoccupied $f$-state peak is found at a higher energy of approximately $E_{\mathrm{F}}+3.5 \mathrm{eV}$. Based on their measurements of the reflection spectra of the rare-earth hexaborides, Kimura et al. also reason, that the energy of the unoccupied $f$-state peak is underestimated in the theoretical calculations and should be at an even higher energy of $E_{\mathrm{F}}+6 \mathrm{eV}$. Furthermore, the inverse photoemission study of $\mathrm{LaB}_{6}$ by Shino et al.[136] suggests an $f$ state peak at $\sim 5.2 \mathrm{eV}$. Although in our simulations with the PBE0r hybrid functional the $f$-state peak is shifted upwards, it is only found at $E_{\mathrm{F}}+2.7 \mathrm{eV}$ at a mixing factor of 0.2 , which is still significantly below the experimentally suggested value of $E_{\mathrm{F}}+6 \mathrm{eV}$. The discussion of this issue is revisited in the discussion of the simulation results of $\mathrm{CeB}_{6}, \mathrm{PrB}_{6}$, and $\mathrm{NdB}_{6}$.

The downward shifts of the La $s$ and $p$ states can be rationalized by the subtraction of the exchange contribution in the PBE0r hybrid functional, which leads to a lowering of the one-particle energies for occupied states. By including the exchange contribution, the unphysical self-interaction, which occurs in the Hartree interaction, is subtracted, c.f. section 3.7. The effect of the selfinteraction correction is large for localized orbitals with a strong interaction, i. e. for the rather core-like La $s$ and $p$ states, which have a rather small spatial extension.

Besides the La $s$ and $p$ states also the La $4 f$ states are localized states and subject to a strong interaction. Because the La $4 f$ states are unoccupied, there is no self-interaction in the $4 f$ shell and no significant change is expected in the $4 f$-projected DOS when switching from the PBE functional to the PBEOr hybrid functional. However, we observe a shift of the unoccupied $4 f$ states to higher energies with increasing exchange mixing factor. We revisit this issue in section 5.9.2, where a rationale for the upward shift of the unoccupied $4 f$ states is given. Note that, as detailed in appendix D, we did not find a change in the size of the relevant Kohn-Sham wave functions which could lead to an increased interaction strength and explain an upward shift of the unoccupied orbitals.

In contrast to the localized lanthanum $5 s$, and $5 p$ states, the rather mobile electrons in the spatially extended boron states largely evade interaction effects. As a consequence, the effect of the self-interaction correction on the boron band is small, which explains the unaltered shape of the boron band with respect to changes in the exchange mixing factor. 


\subsection{Light rare-earth hexaborides}

In $\mathrm{LaB}_{6}$ the effect of the exchange mixing is small because of the lack of occupied strongly localized states. The situation changes for other elements of the lanthanide series, where $f$-electrons are present. With one, two, and three $f$-electrons in their trivalent ionic configuration, respectively [137], cerium, praseodymium, and neodymium are the first three elements of the lanthanides to form rare-earth hexaborides with $f$-orbital occupancy. In the following we present results of DFT simulations of $\mathrm{CeB}_{6}, \mathrm{PrB}_{6}$, and $\mathrm{NdB}_{6}$, for which we used the PBE and the PBE0r exchange-correlation functionals. For the simulations, unit cells of one rare-earth and six boron atoms were used. Unit cell sizes were set according to the bulk lattice constants $a_{\mathrm{Ce}}=4.14 \AA$ [38, 138], $a_{\mathrm{Pr}}=4.13 \AA$ [38], and $a_{\mathrm{Nd}}=4.127 \AA$ [38, 139]. Note, that by using unit cells with only one rare-earth constituent (and one boron octahedron), we exclude antiferromagnetic spin order from our simulations.

If not indicated otherwise, all simulations have been performed with the following parameters. A set of $(2,2,1),(2,2,2,1)$ projector functions is employed for the boron $(s, p, d)$ and rare-earth $(s, p, d, f)$ orbitals, respectively. The complete setups for the atomic species are given in appendix B. The plane wave cutoffs for the plane wave expansions of the pseudo wave functions and the pseudo charge density were set to 50 Ry and 100 Ry, respectively. Furthermore, we used a grid of $(9 \times 9 \times 9) \vec{k}$-points.

\subsubsection{Cerium hexaboride}

In fig. 4.8 we show a comparison of the angular momentum-resolved DOSs of $\mathrm{CeB}_{6}$ that we obtained from a simulation with the PBE-functional and from simulations with the PBE0r-functional with different exchange mixing factors. The same mixing was applied to the boron as well as to the cerium atoms. With the PBE functional the Ce $f$ states are situated in two large peaks, one for each spin direction. The peaks are separated by an energy of $0.4 \mathrm{eV}$ such that the spin symmetry is broken. Moreover, the Fermi level cuts through the lower lying $f$-state peak such that one $f$ orbital is occupied.

When switching to the PBE0r functional a significant change occurs in the projected DOS of the cerium $f$ states. Occupied and unoccupied $f$ states separate and the occupied states form a peak that moves down in energy, whereas the unoccupied states are shifted to higher energies. The separation distance is controlled by the hybrid functional mixing factor and increases with larger values. The shift of the occupied $4 f$ state to a lower energy is expected because the admixture of exchange in the PBE0r hybrid functional aims to correct the self-interaction error and hence lowers the energy of the occupied states. This effect is especially large for strongly localized states, like the cerium $4 f$ and $5 p$ states, because of their large interaction.

Like in our simulations of $\mathrm{LaB}_{6}$, the boron states do not show a reaction to changes of the hybrid functional mixing factor and the valence states match those of the $\mathrm{LaB}_{6}$ simulations. However, at the position of the occupied $f$ 


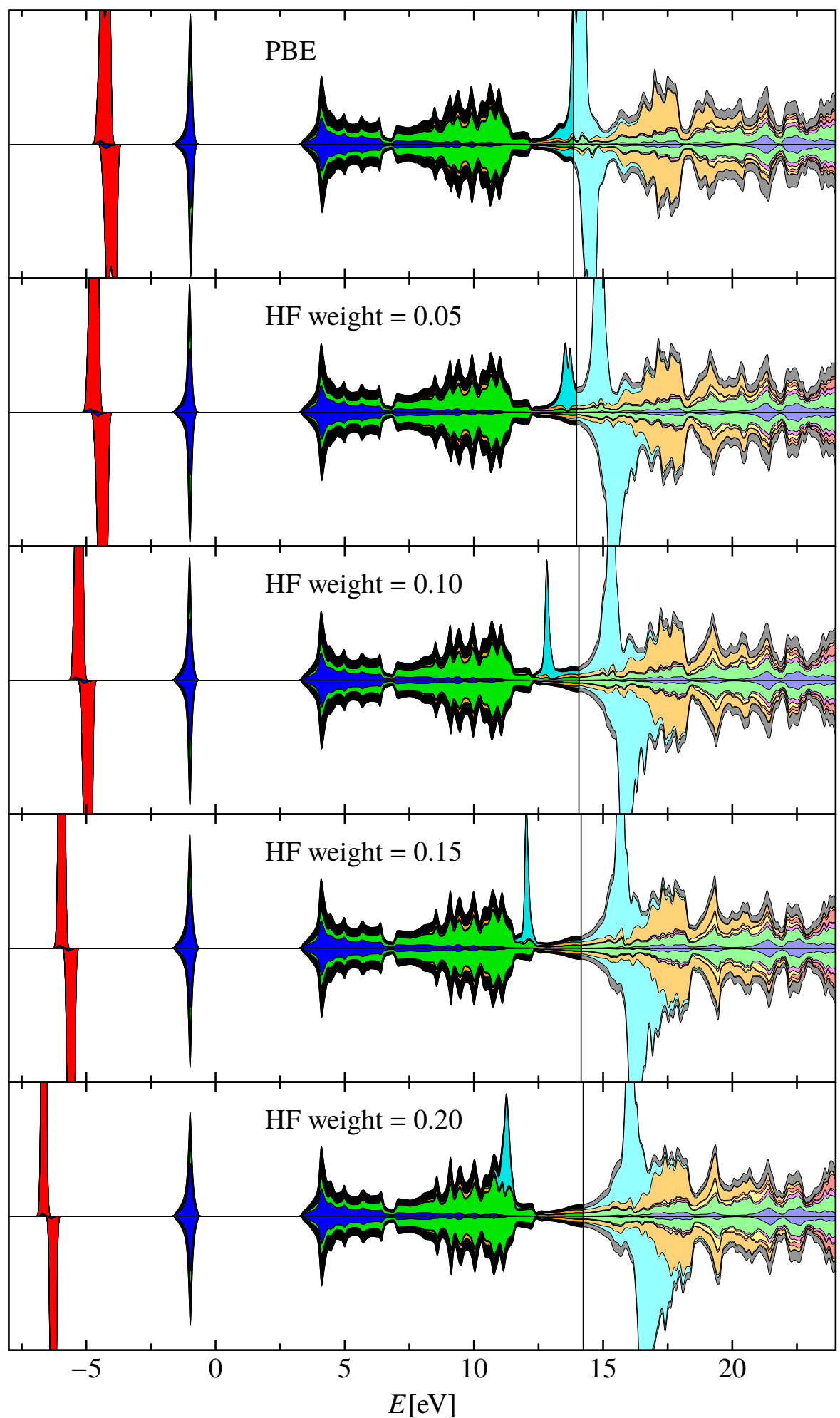

Figure 4.8: Comparison of the DOS of $\mathrm{CeB}_{6}$ from simulations with the PBE functional and the PBE0r functional for multiple mixing factors for the Hartree-Fock exchange. Each graph is split into majority (top) and minority (bottom) spin direction. The Fermi energy is indicated by the black vertical line. Color code: total DOS black, B $s$ blue, B $p$ green, B $d$ magenta, Ce $s$ yellow, Ce $p$ red, Ce $d$ orange, Ce $f$ cyan. 
levels a deformation of the boron DOS occurs which indicates a hybridization between the boron band and the localized lanthanum $f$ states.

In photoemission studies of $\mathrm{CeB}_{6}$ a peak attributed to the $4 f$ electron was found at a binding energy of $\sim 2.1 \mathrm{eV}[30,140]$. The DOS of our simulation of $\mathrm{CeB}_{6}$ at an exchange mixing factor of 0.15 shows the occupied $f$ state at the same energy. Furthermore, a DOS from a previous LSDA $+U$ simulation [133] is available which agrees well with the DOS of our hybrid functional simulations. Specifically, in the earlier data the Ce $4 f$ peak was found at $E_{\mathrm{F}}-2.4 \mathrm{eV}$. An $f$ state at this energy can likely be obtained with an exchange mixing factor of 0.16 .

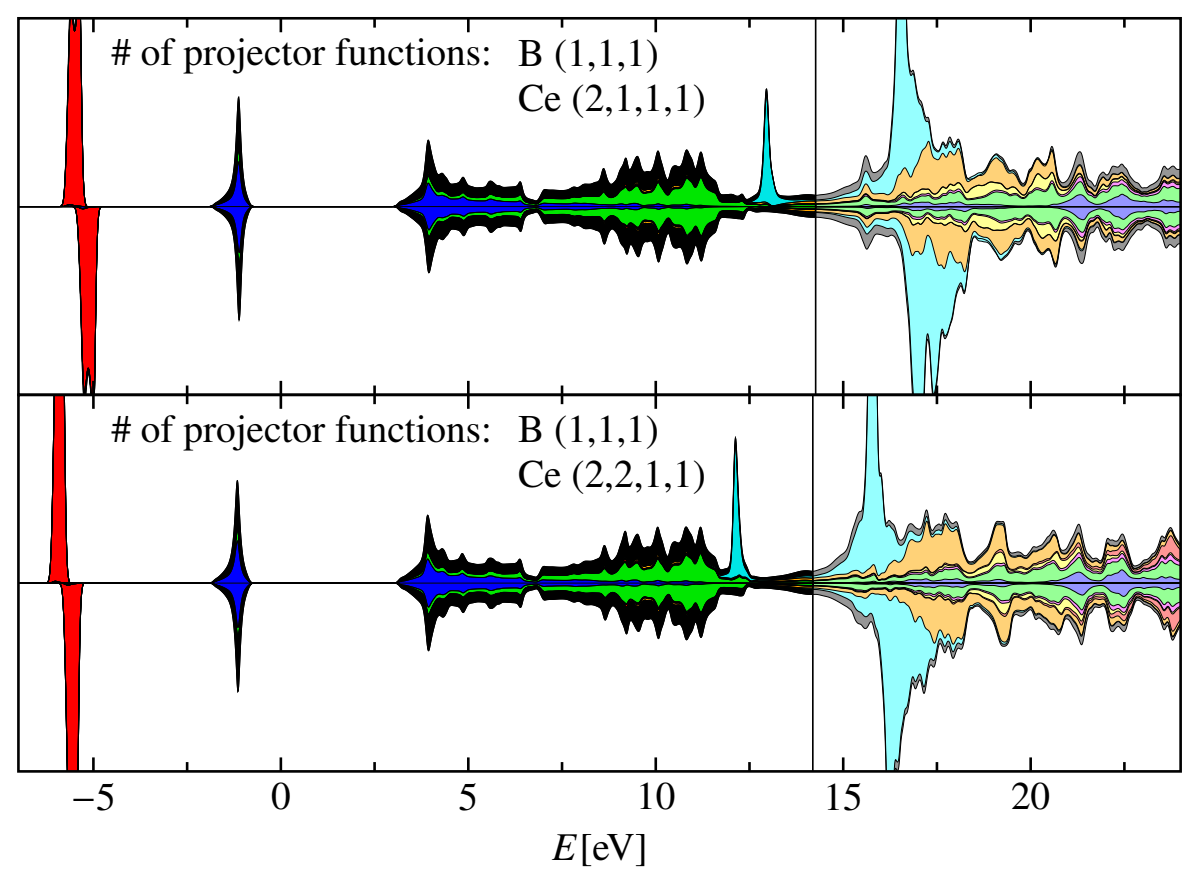

Figure 4.9: $\mathrm{DOS}$ of $\mathrm{CeB}_{6}$ from simulations with the PBE0r hybrid functional at an exchange mixing factor of 0.15 for two different sets of projector functions for the boron $(s, p, d)$ and cerium $(s, p, d, f)$ orbitals. Color code: total DOS black, B $s$ blue, B $p$ green, B $d$ magenta, Ce $s$ yellow, Ce $p$ red, Ce $d$ orange, Ce $f$ cyan.

Like in the results for $\mathrm{LaB}_{6}$, also for $\mathrm{CeB}_{6}$ a small upward shift of the unoccupied $f$ levels is observed. The discussion we gave for the upward shift of the unoccupied $f$ states in $\mathrm{LaB}_{6}$ should also apply here.

In simulations based on the PAW method it is possible that the results to a certain extent depend on the choice for the set of projector functions. This is because the augmentation within the PAW framework relies on the choice for the set of projector functions and partial waves which enter in the computation of the all-electron wave functions and expectation values like the density and the total energy. For $\mathrm{CeB}_{6}$, in fig. 4.9 we show the DOS from simulations with two different sets of projector functions. The two simulations differ in the number of $p$ projector functions for cerium. We included only one $p$ projector function in the simulation of which the DOS is depicted in the top plot and 
two $p$ projector functions in the simulation of which the DOS is depicted in the bottom plot. Evidently, the cerium $f$ orbitals are shifted to lower energies by $0.8 \mathrm{eV}$ when including a second $p$-projector function. Furthermore, the semi-core cerium $s$ (not shown) and $p$ states undergo shifts of $0.5 \mathrm{eV}$ and of $0.4 \mathrm{eV}$ to lower energies, respectively. The reason for this behavior is that both the cerium $5 p$ and $6 p$ states cannot be described adequately in an augmentation with only one partial wave for the cerium $p$ states. However, two partial waves for the cerium $p$ states yield a more suitable basis for the augmentation and the result from the simulations with two $p$ projector functions is considered more accurate. The same reasoning holds for the cerium $s$ states, if the number of $s$-state projector functions is changed. A change of the set of projector functions from $(1,1,1)(2,2,1,1)$ to $(2,2,1)(2,2,2,1)$ projector functions for the boron $(s, p, d)$ and cerium $(s, p, d, f)$ states, respectively, does not alter the result significantly.

\subsubsection{Praseodymium hexaboride}

Like for $\mathrm{CeB}_{6}$, a splitting of the $f$-electron peak is also clearly observed in the DOS of $\operatorname{PrB}_{6}$, which is shown in fig. 4.10 for multiple values of the exchange mixing factor. The $f$-state peak is split into an occupied peak of two electrons, which moves down in energy as the mixing factor is increased, and a peak of unoccupied $f$ states at less than $1.5 \mathrm{eV}$ above the Fermi level. Note that a small portion of $f$ states is always occupied in the majority spin direction in close proximity to the Fermi energy. Moreover, in the DOS for the simulation at an exchange mixing factor of 0.15 a small peak that is located at $E_{\mathrm{F}}+0.2 \mathrm{eV}$ separates from the big peak of unoccupied $f$ states.

We include a simulation of $\operatorname{PrB}_{6}$ at a hybrid functional mixing factor of 0.225 , which places the occupied $f$ states at an energy of $E_{\mathrm{F}}-5 \mathrm{eV}$. This position of the occupied $f$ states is in agreement with photoemission studies of $\operatorname{PrB}_{6}$ by Patil et al. [30], in which a peak assigned to the photo-ionization process $4 f^{2} \rightarrow 4 f^{1}$ was found at a binding energy of $5 \mathrm{eV}$.

Interestingly, in multiple additional simulations of $\mathrm{PrB}_{6}$, differing final states were obtained in the results of simulations with the same hybrid functional mixing factor after convergence of the minimization routine. This was tested in simulations at mixing factors of 0.15 and 0.2 and for different sets of projector functions and $\vec{k}$-point grids. Generally, three different types of converged states were found, which mainly differ in the contribution of the $4 f$ states. We show examples for a DOS of each of these final states in fig. 4.11, which were obtained with a mixing factor of 0.15 . In the following we label them as TYPE I-III in order of appearance within fig. 4.11.

The TyPE I state is close to a spin-degenerate state, where each spin direction has a fully occupied $f$-state peak of one-electron at approximately the same energy of $E_{\mathrm{F}}-3.1 \mathrm{eV}$. In contrast, the TYPE II state has a twofold occupied $f$-state peak at $E_{\mathrm{F}}-3.2 \mathrm{eV}$ in one spin direction only. The TyPE III state possesses a feature of two occupied $f$ states at $E_{\mathrm{F}}-2.8 \mathrm{eV}$ and a partially occupied $f$-state at the Fermi level. In comparison to the TyPE II state, the unoccupied levels are at higher energies in the TYPE III state. According to our 


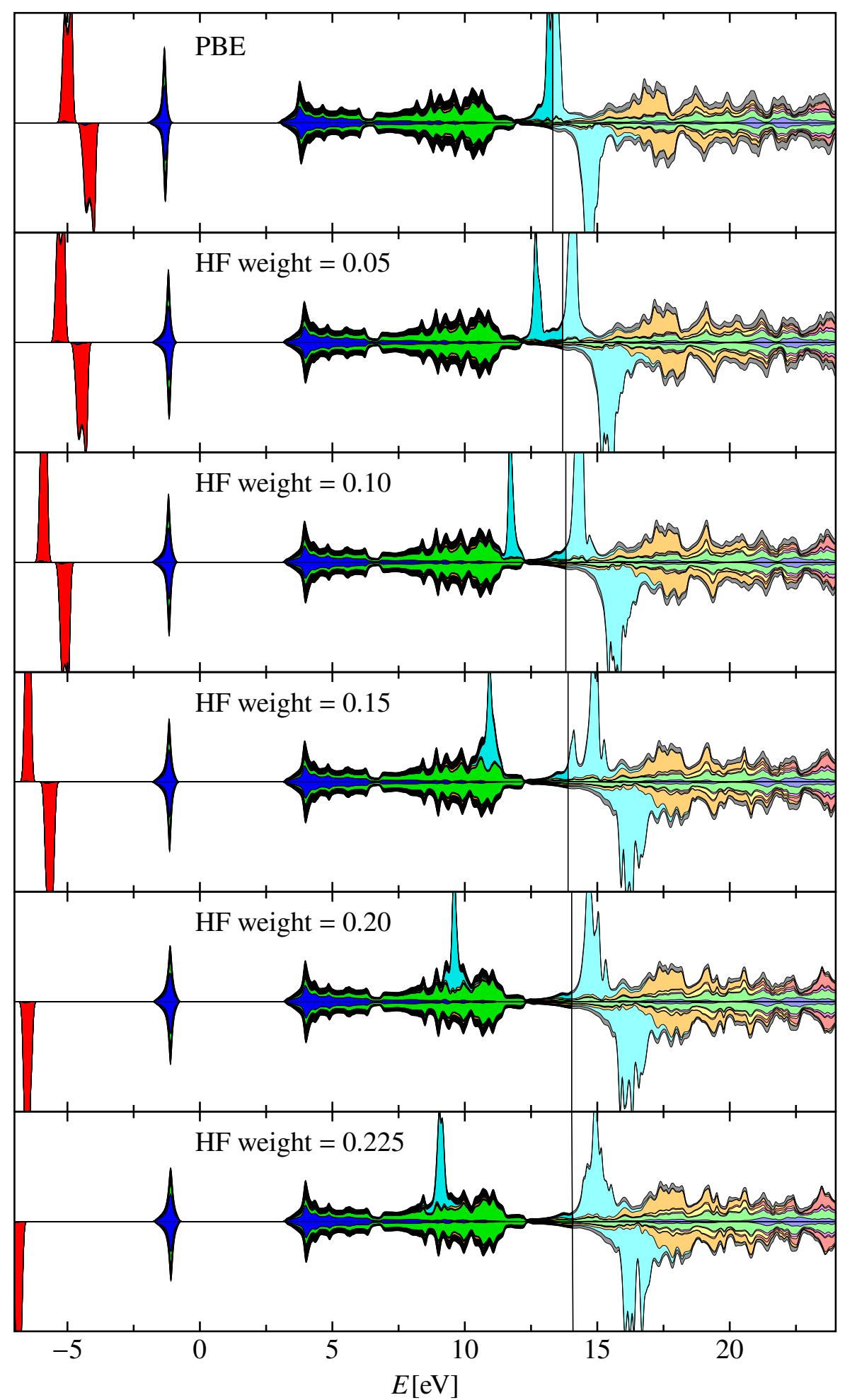

Figure 4.10: Comparison of the DOS of $\mathrm{PrB}_{6}$ from simulations with the PBE functional and the PBE0r functional for multiple mixing factors for the Hartree-Fock exchange. Each graph is split into majority (top) and minority (bottom) spin direction. The Fermi energy is indicated by the black vertical line. Color code: total DOS black, B $s$ blue, B $p$ green, B $d$ magenta, $\operatorname{Pr} s$ yellow, $\operatorname{Pr} p$ red, $\operatorname{Pr} d$ orange, $\operatorname{Pr} f$ cyan. 


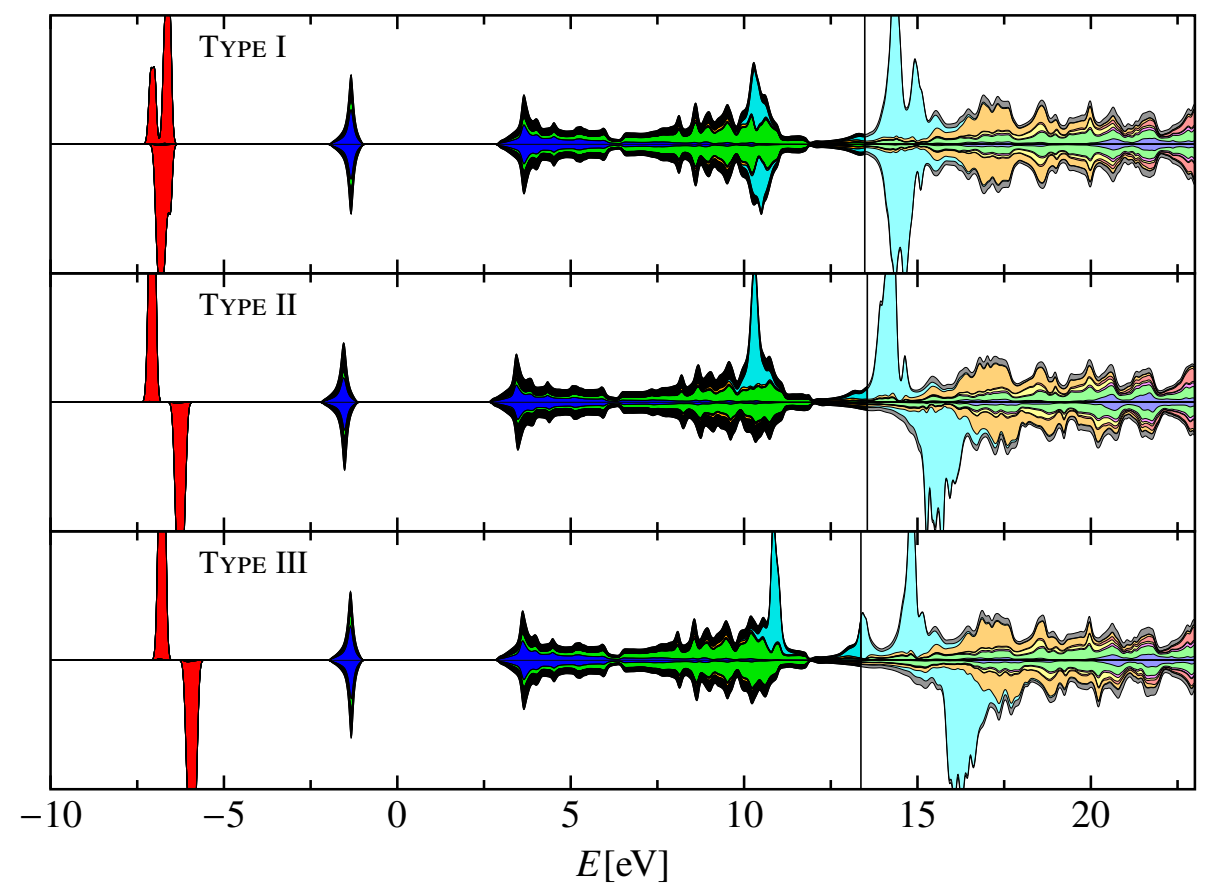

Figure 4.11: Examples of the DOS of the three different final states that were observed after convergence of the minimization routine in multiple simulations of $\operatorname{PrB}_{6}$. All depicted plots were obtained from simulations with $(9 \times 9 \times 9) \vec{k}$-points. The TyPE I and TyPE III DOS examples come from simulations with $(2,2,1)(2,2,2,1)$ projector functions for the boron $(s, p, d)$ and lanthanum $(s, p, d, f)$ orbitals, respectively. The TyPE II example DOS comes from a simulation with $(1,1,1)(2,2,2,1)$ projector functions. The Fermi energies are indicated by the black vertical lines. Color code: total DOS black, B $s$ blue, B $p$ green, B $d$ magenta, $\operatorname{Pr} s$ yellow, $\operatorname{Pr} p$ red, $\operatorname{Pr} d$ orange, $\operatorname{Pr} f$ cyan.

choice of the state labels, in fig. 4.10 the state for a mixing factor of 0.15 is of TYPE III, whereas the states for mixing factors of $0.1,0.2$, and 0.225 are of TYPE II.

The findings of three differing stable final states of the minimization procedure indicate the existence of at least three minima on the potential landscape of the simulation. The ground state corresponds to the global minimum of the potential landscape. A state that corresponds to a local but not global minimum and has an energy well above the global minimum can be discarded from the ground state search. Hence, to identify which states correspond to local but not global minima, the energies of each of the three states need to be compared for the same set of simulation parameters.

We set up a series of simulations at a mixing factor of 0.15 , where we aimed to address each state systematically for a selected $\vec{k}$-point grid and a selected set of projector functions. $\vec{k}$-point grids of $(N \times N \times N) \vec{k}$-points, where $N \in\{7,9,11,13\}$, were considered as well as the following sets of projector functions for the boron $(s, p, d)$ and the praseodymium $(s, p, d, f)$ orbitals: 


$$
(1,1,1)(2,1,1,1) ;(1,1,1)(2,2,1,1) ;(1,1,1)(2,2,2,1) \text {; and }(2,2,1)(2,2,2,1) \text {. }
$$

Note, that the result of a simulation can be reused as a starting point of a successive simulation in which the set of projector functions is changed. However, it is not possible to continue a simulation with a different $\vec{k}$-point grid. In the following we describe our course of action that was repeated for each of the $\vec{k}$-point grids.

As a starting point we chose the converged atomic structure of a previous simulation and performed a relaxation of the wave functions and the atomic structure in a spin-degenerate simulation for a set of $(1,1,1)(2,1,1,1)$ projector functions. From the final state of this simulation the TyPE I states for each set of projector functions are obtained in subsequent simulation runs, after adjusting the number of projector functions accordingly and allowing for spinpolarization. A small temporary spin-polarized potential was applied to the $\mathrm{Pr}$ $f$ orbitals to lift the spin-degeneracy. This auxiliary potential is only active for a few simulation steps and is deactivated when we let the system converge towards a minimum of the potential landscape. Note that the converged TYPE I state is not completely symmetric in the spin. To proceed from the TYPE I states, a rather strong auxiliary spin-polarized potential was applied temporarily to the $\operatorname{Pr} f$ orbitals to lift the near-spin-degenerate state. After deactivating the auxiliary potential, the minimization routine led to either a TYPE II or Type III state. It was then tried to find the missing state for each set of projector functions by performing a simulation, starting from the result of a different set of projector functions, for which the missing state was already obtained. To prevent the system from leaving the selected state after changing the set of projector functions, a large initial friction was applied in the equation of motion of the wave functions in the Car-Parrinello computational scheme. However, this does not guarantee that the simulation will stay in the desired state as the simulation can either leave the selected minimum on the potential landscape, or the minimum could be lifted for the new set of projector functions. Indeed not all states were addressed successfully for the different sets of projector functions. The total energies for the final states of the minimization procedure are given in appendix $\mathrm{C}$ for all parameter sets.

With the procedure described above, we found stable states for all tested $\vec{k}$-point grids and all tested sets of projector functions, with the following exceptions: for all $\vec{k}$-point sets no stable TYPE III state was obtained for the set of $(1,1,1)(2,1,1,1)$ projector functions and no stable TyPE II state was found for the set of $(2,2,1)(2,2,2,1)$ projector functions. Furthermore, for the simulations with $(1,1,1)(2,2,1,1)$ projector functions, the TYPE III state was only found for the $\vec{k}$-point grids of $(7 \times 7 \times 7)$ and $(9 \times 9 \times 9) \vec{k}$-points.

The missing of a stable TYPE III state for the set of $(1,1,1)(2,1,1,1)$ projector functions and of a stable TyPE II state for the set of $(2,2,1)(2,2,2,1)$ projector functions can be rationalized as follows. In the PAW method the computation of expectation values, such as the density and the total energy, depends on the set of projector functions used in the augmentation as described in section 3.11.2. Since, in DFT, the potential depends on the density and, moreover, we search for a minimum of the total energy, it is possible that for certain sets of projector functions the minimum of the total energy which corresponds to the missing 
state is lifted. Following the arguments given above, the dependence of the total energy on the set of projector functions is also a plausible explanation for small differences in the total energy of final states of the same type for different sets of projector functions.

For parameter sets, for which a TyPE II state as well as a TyPE III state was obtained, a comparison of the total energies of both states showed a largest difference of less than $4 \mathrm{meV}$, which is negligible in the context of our simulations. In comparison with the TyPE II and TyPE III states, the TyPE I state was always found at a larger energy with energy differences of $\sim 0.43 \mathrm{eV}$ to $\sim 0.48 \mathrm{eV}$. Thus, due to its larger value for the total energy, a Type I state correspond to a local but not global minimum of the potential landscape and cannot be the ground state. This finding is in accordance with Hund's second rule, which states that the total spin of all electrons in the $f$ shell should be maximized in the ground state. The approximately equal energies of the TYPE II and TYPE III states indicate a degenerate ground state, where a ground state of the simulation is a linear combination of the TYPE II and TYPE III states.

We also tested the dependence of the TYPE I-III states and their energies on the number of $\vec{k}$-points. The largest difference in the total energy for states of the same type at the same set of projector functions was less than $5 \mathrm{meV}$. A small difference is expected when selecting a different $\vec{k}$-point grid.

For grids of $(11 \times 11 \times 11)$ and $(13 \times 13 \times 13) \vec{k}$-points, the TYPE III state was not found to be stable for a set of $(1,1,1)(2,2,1,1)$ projector functions, whereas it was successfully addressed for grids of $(7 \times 7 \times 7)$ and $(9 \times 9 \times 9) \vec{k}$-points. To find the missing state, we started from the converged simulation for the TYPE III state from a set a $(1,1,1)(2,2,2,1)$ projector functions and changed the set of projector functions to $(1,1,1)(2,2,1,1)$. A TYPE III-like state can be observed in the simulation for only a few steps. However, it is not found to be stable and slowly transitions into a TYPE II state when continuing the simulation until convergence. If the potential landscape is rather flat in the vicinity of the TYPE II and TYPE III states with only a shallow TYPE III minimum for grids of $(7 \times 7 \times 7)$ and $(9 \times 9 \times 9) \vec{k}$-points, the change to a grid of $(11 \times 11 \times 11)$ or $(13 \times 13 \times 13) \vec{k}$-points could be enough for the minimum to be lifted.

\subsubsection{Neodymium hexaboride}

As shown in fig. 4.12, for $\mathrm{NdB}_{6}$ a splitting of the $f$ electron peak into a three peak structure is observed when switching from the PBE functional to the PBE0r hybrid functional. The lower $f$-state peak accommodates three electrons. An $f$-state peak with a fractional occupancy, which can host a total of one electron, is pinned to the Fermi level for all values of the exchange mixing factor. At mixing factors $\geq 0.15$ this peak is well separated from the fully occupied and completely unoccupied $f$-state peaks. The three peak structure of the $f$-state projected DOS is similar to that of the $f$-state projected DOS of the TYPE III state, that was found in the simulations of $\operatorname{PrB}_{6}$. In contrast to the simulations of $\mathrm{PrB}_{6}$, for $\mathrm{NdB}_{6}$ only one stable state was found for each set of parameters. Moreover, in our simulations of $\mathrm{NdB}_{6}$, only a small downward shift of the fully occupied $f$ states is found when increasing the hybrid functional exchange 


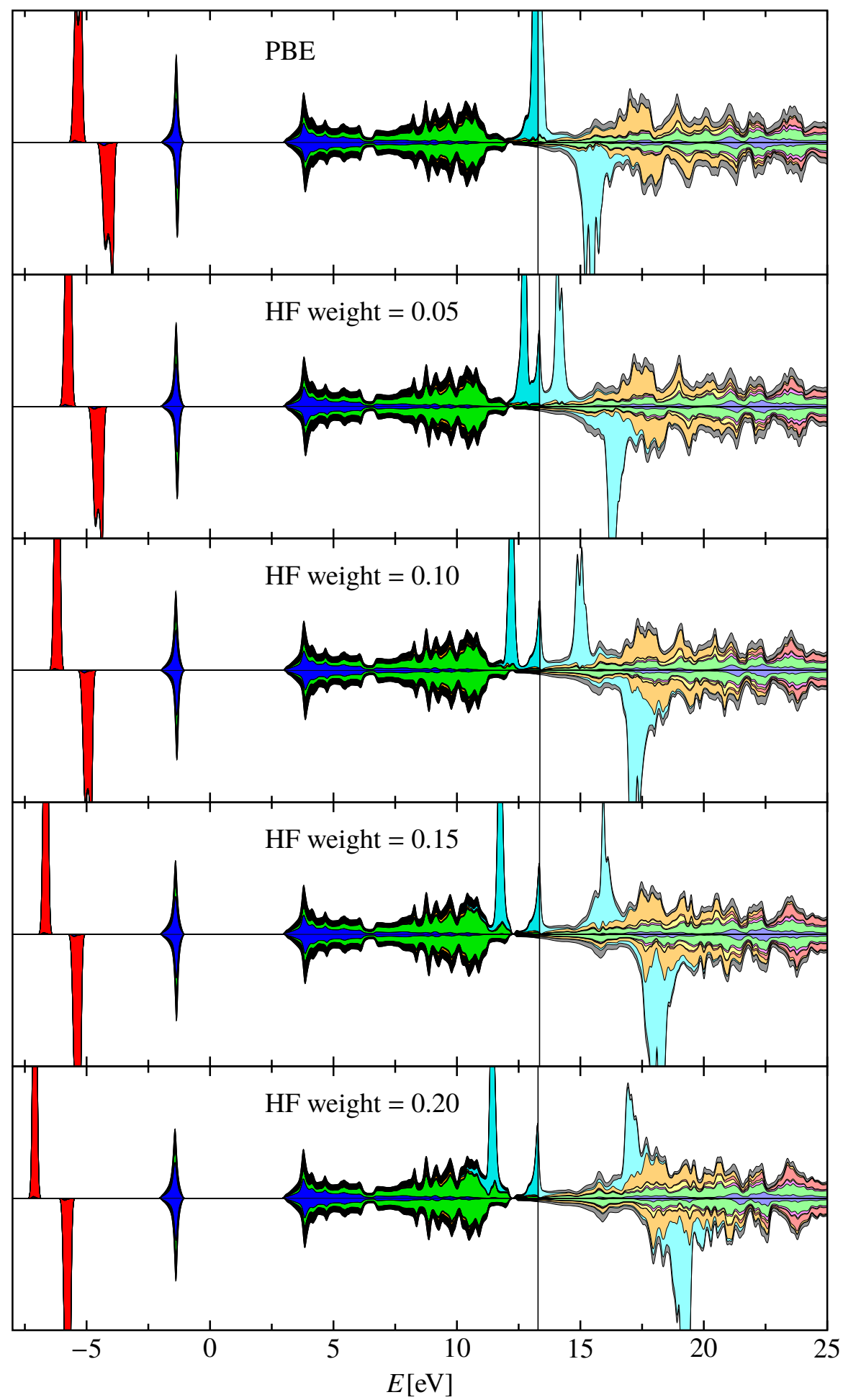

Figure 4.12: Comparison of the DOS of $\mathrm{NdB}_{6}$ from simulations with the $\mathrm{PBE}$ functional and the PBE0r functional for multiple mixing factors for the Hartree-Fock exchange. Each graph is split into majority (top) and minority (bottom) spin direction. The Fermi energy is indicated by the black vertical line. Color code: total DOS black, B $s$ blue, B $p$ green, B $d$ magenta, $\mathrm{Nd} s$ yellow, $\mathrm{Nd} p$ red, $\mathrm{Nd} d$ orange, $\mathrm{Nd} f$ cyan. 
mixing factor, but a larger upward shift of the unoccupied $f$ states occurs in comparison to our simulations of $\mathrm{CeB}_{6}$ and $\mathrm{PrB}_{6}$. Because of this the occupied $f$ levels are located at a much higher energy than found experimentally.

In photoemission experiments on $\mathrm{NdB}_{6}$ a peak for the photo-ionization process $4 f^{3} \rightarrow 4 f^{2}$ was found at a binding energy of $7 \mathrm{eV}$ [141] or $6.25 \mathrm{eV}$ [30], respectively. Furthermore, an $\mathrm{LSDA}+U$ simulation of $\mathrm{NdB}_{6}$ by Singh et $a l$. showed an $f$-state peak at $E_{\mathrm{F}}-7 \mathrm{eV}$ [133]. The DOS of our simulations in fig. 4.12 is not in agreement with the previous data, as the fully occupied $f$ states are situated much closer to the Fermi level. We also performed simulations of $\mathrm{NdB}_{6}$ with larger exchange mixing factors between 0.2 and 0.4 . However, none of our simulations resulted in a DOS with an $f$ state peak below an energy of $E_{\mathrm{F}}-2.5 \mathrm{eV}$. Instead, simulations at very strong exchange mixing factors of 0.3 and above result in the fractionally occupied peak at the Fermi level becoming fully occupied and merging with the other occupied orbitals right below the Fermi energy.

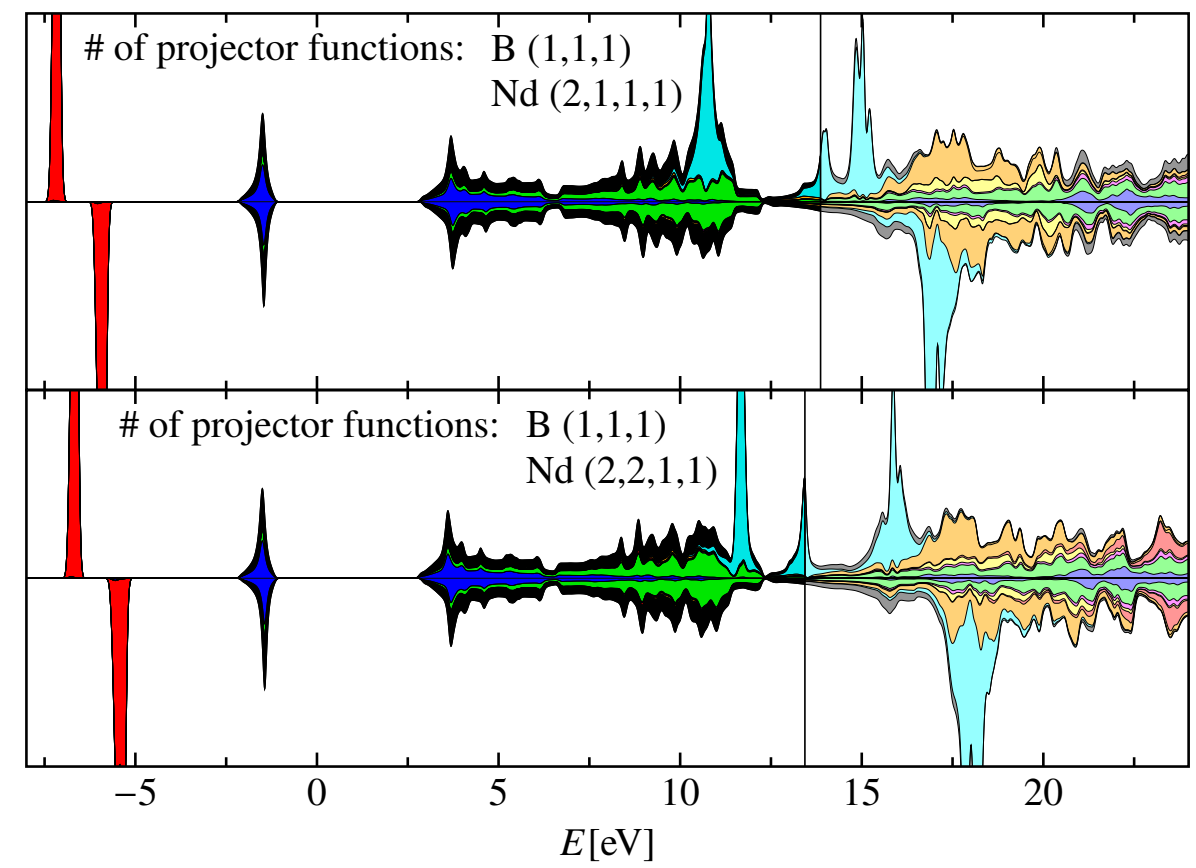

Figure 4.13: DOS of $\mathrm{NdB}_{6}$ from simulations with the PBEOr hybrid functional at an exchange mixing factor of 0.15 for two different sets of projector functions for the boron $(s, p, d)$ and neodymium $(s, p$, $d, f)$ orbitals. Color code: total DOS black, B $s$ blue, B $p$ green, $\mathrm{B} d$ magenta, $\mathrm{Nd} s$ yellow, $\mathrm{Nd} p$ red, $\mathrm{Nd} d$ orange, $\mathrm{Nd} f$ cyan.

Like for $\mathrm{CeB}_{6}$, in fig. 4.13 we compare the DOS from simulations with sets of $(1,1,1)(2,1,1,1)$ and $(1,1,1)(2,2,1,1)$ projector functions. Here, the fully occupied and completely empty neodymium $4 f$ states are shifted upwards by $0.9 \mathrm{eV}$ when changing from the set of $(1,1,1)(2,1,1,1)$ projector functions to the set of $(1,1,1)(2,2,1,1)$ projector functions. Note, that the distance of the two peaks is not changed. The fractionally occupied state at the Fermi level shows a different behavior as it is shifted downwards by $0.55 \mathrm{eV}$ and the $4 f$ 
occupancy is increased from 3.37 to 3.63. An explanation for this behavior is given in chapter 5 . The energy levels of the neodymium $5 s$ and $5 p$ levels are shifted upwards in energy by $0.4 \mathrm{eV}$, and $0.5 \mathrm{eV}$, respectively.

Again, the bottom state should be more accurate because a basis with two partial waves for the neodymium $p$ states in the augmentation within the PAW method is more suitable to represent the $5 p$ and $6 p$ states than a basis with only one $p$-state partial wave. No significant change in the DOS is observed when switching from $(1,1,1)(2,2,1,1)$ projector functions to a set of $(2,2,1)(2,2,2,1)$ projector functions.

\subsubsection{Dependence of the $f$-state energies on the exchange mix- ing factor}

In fig. 4.14 we plot the positions of the $4 f$ state peaks from all DOSs shown in fig. $4.8\left(\mathrm{CeB}_{6}\right)$, fig. $4.10\left(\mathrm{PrB}_{6}\right)$, and fig. $4.12\left(\mathrm{NdB}_{6}\right)$ against the exchange mixing factor of the PBE0r hybrid functional. For the energy level of the occupied orbitals of $\mathrm{CeB}_{6}$ a linear decline with increasing exchange mixing is found. This is expected since an increase in the exchange mixing factor leads to a proportionally larger self-interaction term that is subtracted from the orbital energy. The monotonous growth of the energy of the unoccupied orbitals with larger exchange mixing is likely due to the same reason as in $\mathrm{LaB}_{6}$, see the discussion in section 4.3.3. A rationale is given in section 5.9.2.

In the case of $\operatorname{PrB}_{6}$ the DOSs are mostly of TYPE II and the energy level of the occupied states relative to the Fermi level also decreases linearly with increasing mixing factor. The steeper slope in comparison to the data of $\mathrm{CeB}_{6}$ is expected, since a larger interaction in the $\operatorname{Pr} 4 f$ shell should result in a stronger effect of the self-interaction compensation. A deviation in the otherwise linear decline is the DOS for an exchange mixing of 0.15 , which is of TYPE III. The slight elevation from the expected energy at 0.15 exchange mixing is also observed in the data of the unoccupied states of $\operatorname{PrB}_{6}$.

The different behavior of the energy levels in a TYPE III DOS is clear in the data of the $4 f$ energy levels for $\mathrm{NdB}_{6}$. As $\mathrm{Nd}$ should have the strongest interaction in the $4 f$ shell, an even steeper decline in the energy of the occupied $4 f$ levels with increasing exchange mixing factor is expected. Instead the smallest and less-than-linear decrease is found, while the energies of the unoccupied states grow faster than in $\mathrm{CeB}_{6}$ and $\mathrm{PrB}_{6}$.

From the the data of fig. 4.14 we obtain a linear dependence of the distance between the fully occupied and unoccupied $4 f$ levels for $\mathrm{CeB}_{6}, \mathrm{PrB}_{6}$, and $\mathrm{NdB}_{6}$, as depicted in fig. 4.15. We do not comment on this data at this point, but postpone the discussion to chapter 5 . In chapter 5 we explain the shape of $4 f$-projected DOS of the light rare-earth hexaborides, as found by DFT with the PBE0r hybrid functional, on the basis of a simple model system. In this context the data of figs. 4.14 and 4.15 are used to compare the DFT DOSs and the DOS of the model system. A detailed rationale for the dependence of the peak positions in fig. 4.14 on the exchange mixing factor is given in section 5.9.2. 


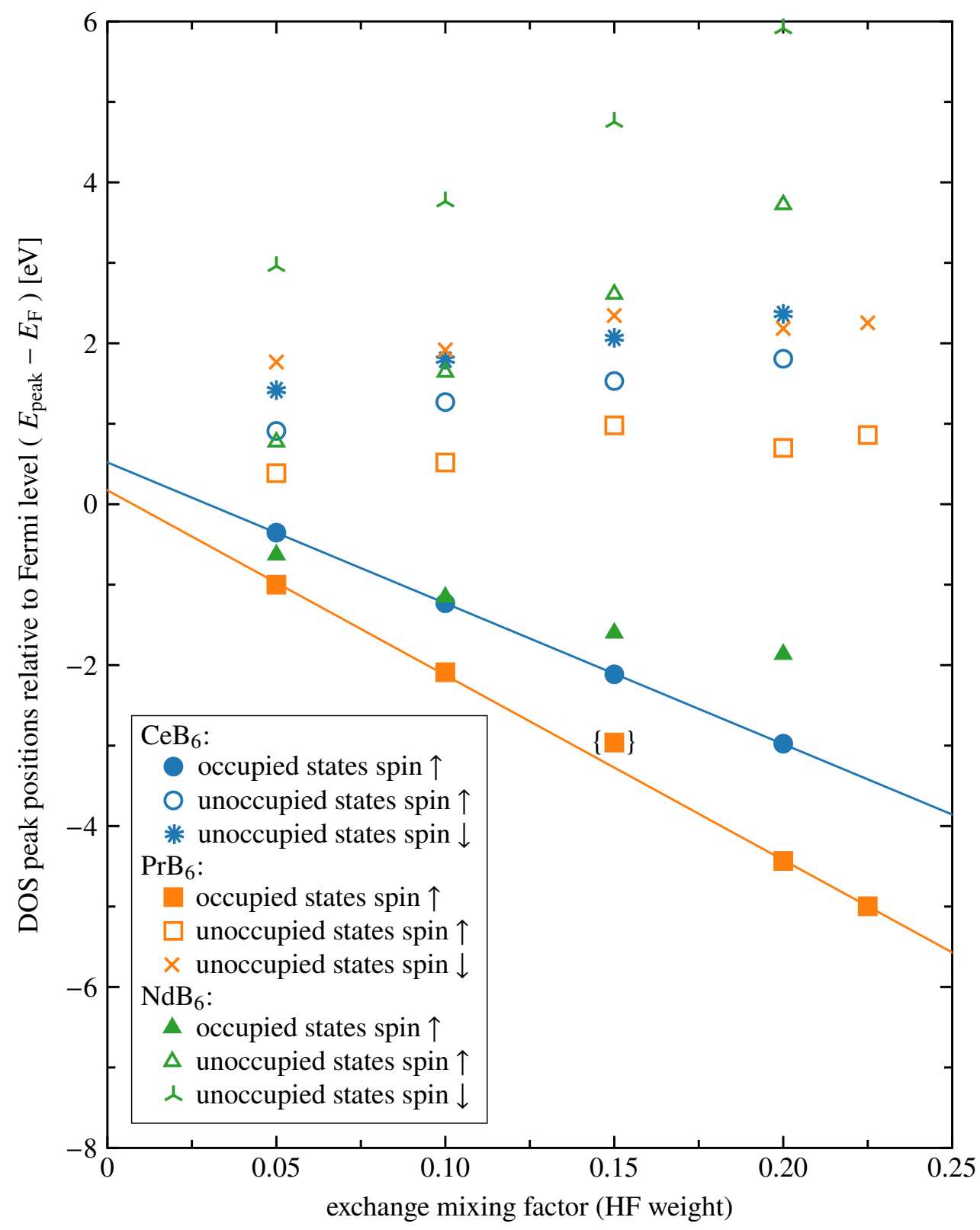

Figure 4.14: Energies of the fully occupied and empty $4 f$ states relative to the Fermi level against the hybrid functional mixing factor (HF weight) for $\mathrm{CeB}_{6}, \mathrm{PrB}_{6}$, and $\mathrm{NdB}_{6}$. The data point at $\mathrm{HF}$ weight 0.15 for $\mathrm{PrB}_{6}$ is excluded from the linear fit, see text. Error bars are omitted for readability. The largest errors due to read-off imprecision are: $0.1 \mathrm{eV}$ for occupied states, spin $\uparrow ; 0.2 \mathrm{eV}$ for unoccupied states, spin $\uparrow ; 0.3 \mathrm{eV}$ for unoccupied states, spin $\downarrow$.

Shino et al. performed inverse photoemission measurements based on X-ray bremsstrahlung isochromat spectroscopy (X-BIS) on several of the rare-earth hexaborides [136]. Their X-BIS data show broad features at $E_{\mathrm{F}}+(4.3 \pm 0.4) \mathrm{eV}$ for $\mathrm{CeB}_{6}$ and at $E_{\mathrm{F}}+(4.0 \pm 0.4) \mathrm{eV}$ for $\mathrm{PrB}_{6}$. Since the X-BIS measurements are not spin-sensitive, for a comparison we use the center position between the unoccupied energy levels of both spin direction from our DOS data. We find 


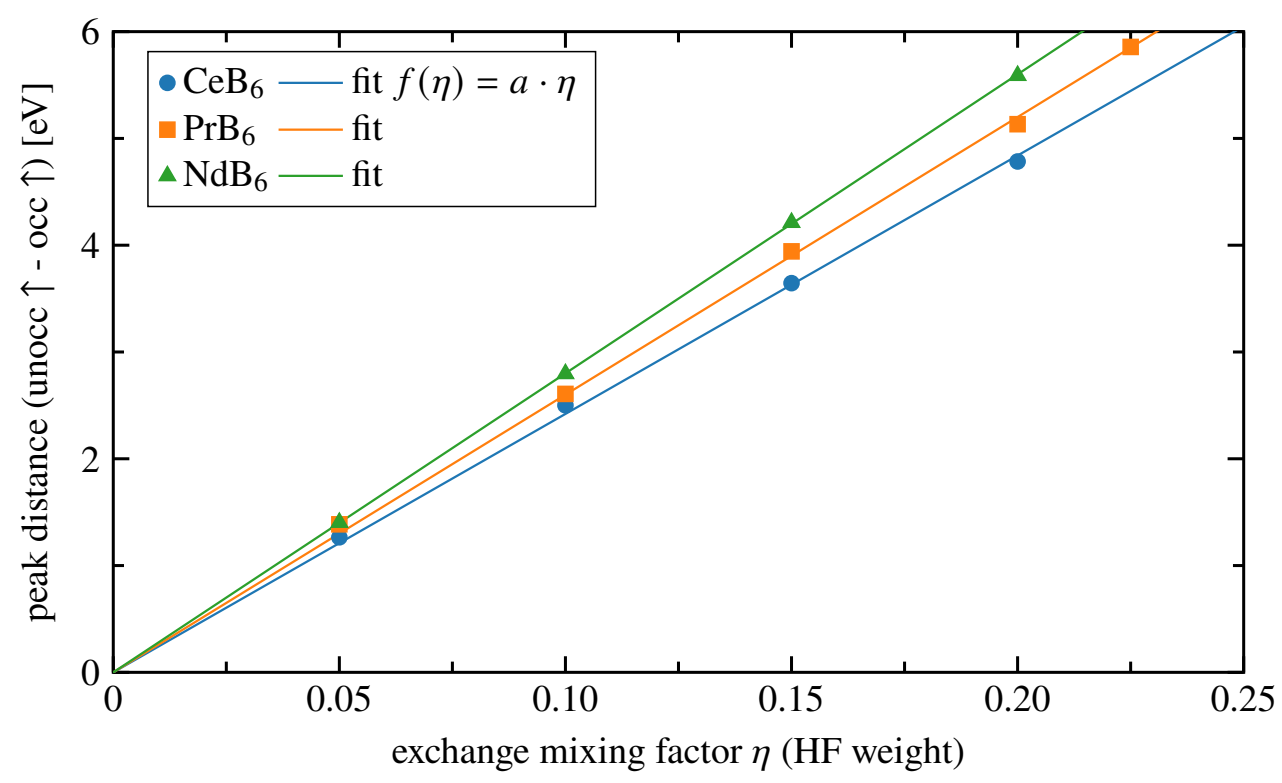

Figure 4.15: Dependence of the energy distance between the fully occupied states and the empty states of the majority spin direction on the hybrid functional mixing factor (HF weight) for $\mathrm{CeB}_{6}, \mathrm{PrB}_{6}$, and $\mathrm{NdB}_{6}$. Error bars are omitted for readability. The largest error due to read-off imprecision of the peak positions is $0.21 \mathrm{eV}$.

$E_{\mathrm{BIS}, \mathrm{Ce}}=E_{\mathrm{F}}+(1.80 \pm 0.12) \mathrm{eV}$ for $\mathrm{CeB}_{6}$ at an exchange mixing factor of 0.15 and $E_{\mathrm{BIS}, \mathrm{Pr}}=E_{\mathrm{F}}+(1.56 \pm 0.2) \mathrm{eV}$ for $\mathrm{PrB}_{6}$ at an exchange mixing factor of 0.225 . The error is propagated from the read-off imprecision of the $4 f$ features in the DOS. It is clear, that in comparison to the X-BIS data, the unoccupied states in the DOSs from our DFT simulations appear $\sim 2.5 \mathrm{eV}$ lower in energy. We are aware that discrepancies between calculated DOSs and experimental BIS spectra have been observed before for transition- and noble-metal elements [142]. However, in this earlier study, features of the DOS appeared consistently at lower energies in comparison to features of the BIS data only at energies above $10 \mathrm{eV}$.

In their study of the electronic states of the rare-earth hexaborides via measurements of reflectivity spectra, also Kimura et al.[135] suggest that the energy of the unoccupied rare-earth hexaboride $4 f$ states is underestimated in bandstructure calculations. We are not aware of a satisfactory explanation for discrepancies of the observed size between the DOSs of DFT calculations and the BIS and reflectivity data. However, one should be aware that although the DOS of the Kohn-Sham system is often interpreted as the one-particle excitation spectrum of the physical interacting system, no theoretical justification for this interpretation has been given (see e. g. [143]). With that in mind, it is reasonable to expect discrepancies between experimentally measured excitation spectra and the DOS of the Kohn-Sham system. 


\subsection{5 $f$-state occupancy at an exchange mixing factor of $\mathbf{0 . 1 5}$}

The presence of a fractionally occupied $4 f$ state at $E_{\mathrm{F}}$ in the TyPE III DOSs of $\mathrm{PrB}_{6}$ and the DOSs of $\mathrm{NdB}_{6}$ results in a fractional occupancy of the respective $f$-shell. This is elaborated in fig. 4.16, where the projected DOSs of the $f$ states and the integrated $f$-level DOSs of $\mathrm{CeB}_{6}, \mathrm{PrB}_{6}$, and $\mathrm{NdB}_{6}$ are shown for an exchange mixing of 0.15 . In the case of $\mathrm{CeB}_{6}$ the $f$-level occupancy is close to 1 . For $\operatorname{PrB}_{6}$ and $\mathrm{NdB}_{6}$ the $f$-shell has a fractional occupancy of 2.35 for $\mathrm{PrB}_{6}$ and 3.63 for $\mathrm{NdB}_{6}$.

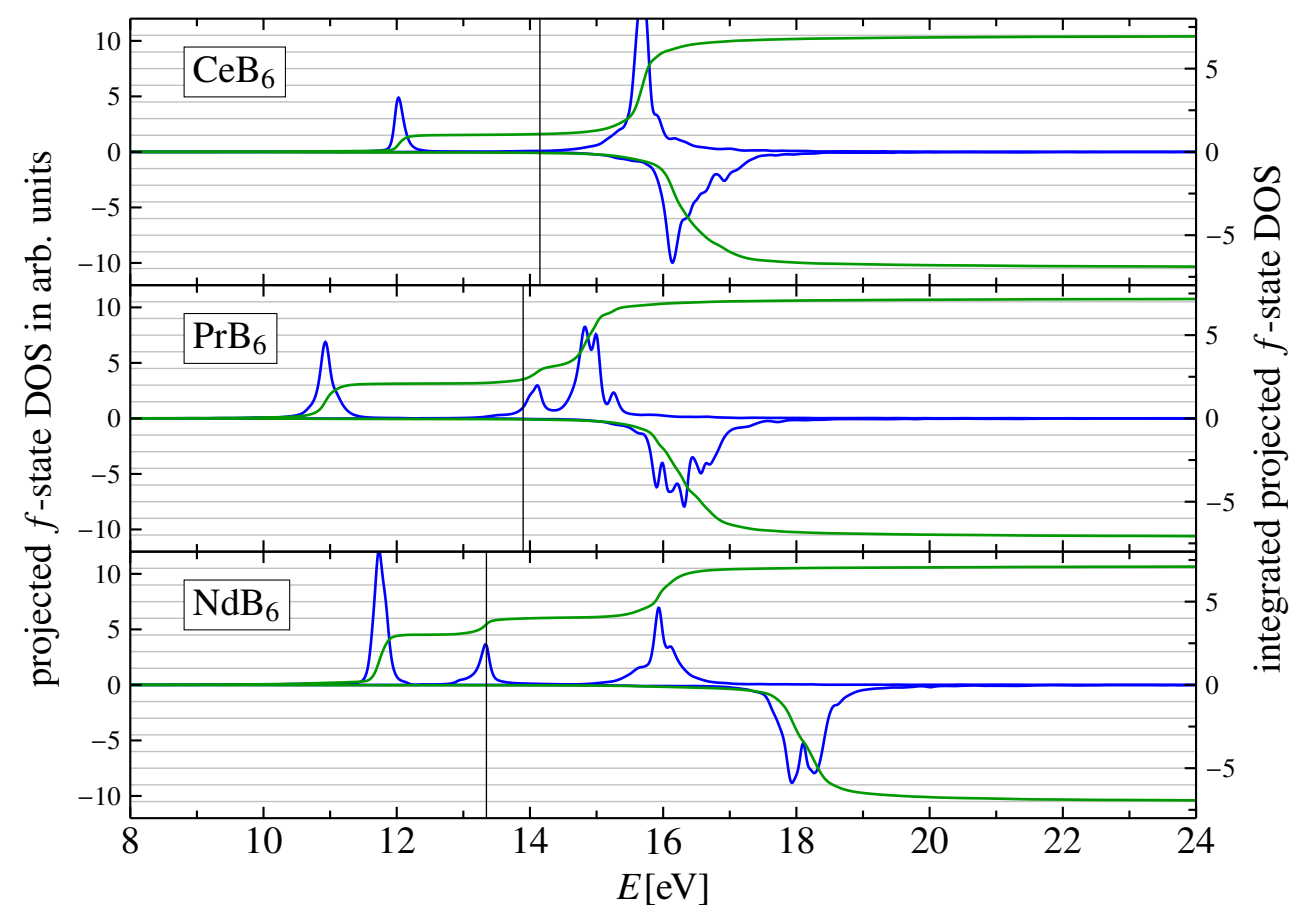

Figure 4.16: Projected DOS (blue, left axis) and integrated projected DOS (green, right axis) of the $f$-orbitals of $\mathrm{CeB}_{6}, \mathrm{PrB}_{6}$, and $\mathrm{NdB}_{6}$ as obtained from the PBE0r hybrid functional DFT simulation with an exchange mixing factor of 0.15 . The grey horizontal lines indicate integer values of the integrated projected DOS. The Fermi levels are marked with the black vertical lines.

\subsection{Discussion}

In this chapter we present results of bulk electronic structure calculations on the light rare-earth hexaborides $\mathrm{LaB}_{6}, \mathrm{CeB}_{6}, \mathrm{PrB}_{6}$, and $\mathrm{NdB}_{6}$, based on DFT together with the PAW method. We started our study of the rare-earth hexaborides with simulations of the boron network. Our simulation results show that the $\left[\mathrm{B}_{6}\right]^{2-}$ crystal with a homogeneous positive charge background is an insulator with an energy gap of $1.8 \mathrm{eV}$. The occupied states are of bonding-type tight-binding orbitals of the boron octahedra and octahedronoctahedron bonds, while the unoccupied orbitals comprise the antibonding- 
type tight-binding orbitals. These results agree with previous theoretical work by Longuet-Higgins and Roberts [132].

The shape of the DOS projected onto the bonding boron tight-binding orbitals carries over into the DOS of our simulations on $\mathrm{LaB}_{6}$. In contrast to the $\left[\mathrm{B}_{6}\right]^{2-}$ crystal, $\mathrm{LaB}_{6}$ is shown to be metallic as the energy gap is closed by the antibonding boron states stretching below the Fermi level, and the lanthanum $5 d e_{g}$ states. The change from an insulator in the $\left[\mathrm{B}_{6}\right]^{2-}$ crystal to a metal in $\mathrm{LaB}_{6}$ is attributed to the additional electron in the $\mathrm{LaB}_{6}$ unit cell. The projected DOS of the boron states does not appear to be altered significantly when moving on to $\mathrm{CeB}_{6}, \mathrm{PrB}_{6}$, or $\mathrm{NdB}_{6}$.

The light rare-earth hexaborides $\mathrm{CeB}_{6}, \mathrm{PrB}_{6}$, and $\mathrm{NdB}_{6}$ possess occupied $4 f$-orbitals, which are strongly localized states, coupled via a hybridization to the metallic states of the material. Typically, such systems are not well described by traditional functionals within the local spin density approximation or generalized gradient approximation. With the aim to improve upon the unsatisfactory results from LSDA or GGA functionals, we assess the applicability of the PBE0r local hybrid functional to the light rare-earth hexaborides. In the PBE0r hybrid functional the mixing of the exchange part of the traditional PBE functional with a portion of exact exchange results in a correction of the selfinteraction error, which is present in simulations of interacting systems with LSDA and GGA functionals. This error can lead to stark disagreements with experimental results, especially when the material contains strongly localized orbitals.

The main impact of the hybrid functional can be understood in analogy to the Hartree-Fock approximation. In the Hartree-Fock approximation the electron-electron interaction is comprised of a Hartree term and an exchange term. The exchange compensates the interaction of an electron with itself, which is present within the Hartree term. As a consequence, an electron in an occupied orbital experiences an interaction with one less electron than an electron that would be inserted into an unoccupied orbital in the system. Hence, the occupied and unoccupied levels are separated by an energy of the size of the interaction strength. If the self-interaction were not compensated, the energy separation between occupied and unoccupied states would vanish. Since the admixture of exact exchange in the PBE0r hybrid functional introduces a better compensation of the self-interaction in DFT, a downward shift of the occupied Kohn-Sham states is expected. The shift size is in proportion to the interaction strength between the localized orbitals and is larger, the greater the overlap between a Kohn-Sham state and a state of the local basis.

For $\mathrm{LaB}_{6}$ our results with the PBE0r hybrid functional show that mainly the semi-core $5 s$ and $5 p$ states are affected by a downward shift in energy. This is plausible since these states are fairly localized and are affected by a rather large interaction. In contrast, the other occupied states are mainly boron states, which do not show a reaction to changes of the exchange mixing factor. This can be rationalized by their rather large spatial extension, leading to a rather small interaction and a negligible effect of the exchange mixing.

In $\mathrm{CeB}_{6}$, one of the strongly localized $4 f$ states is occupied. The exchange mixing of the PBE0r hybrid functional induces an energy split between the 
occupied state and the unoccupied states in the majority spin direction which can be observed in the DOS. At an exchange mixing of 0.15 the occupied $4 f$ state is found at an energy of $E_{\mathrm{F}}-2.1 \mathrm{eV}$ in accordance with photoemission studies $[30,140]$. At this exchange mixing factor the energy separation of the occupied and unoccupied $4 f$ states, which is a measure for an effective interaction strength in the $f$ shell, is $3.6 \mathrm{eV}$. This is smaller than a previous estimate for the interaction strength of around $5 \mathrm{eV}$, based on calculations on the neutral lanthanide atoms [137].

Downward shifts of the the occupied $4 f$ states are also observed in simulations of $\mathrm{PrB}_{6}$, and $\mathrm{NdB}_{6}$. However, in comparison with the simulations of $\mathrm{CeB}_{6}$, finding the ground state of $\mathrm{PrB}_{6}$ is more difficult, as three different stable states have been identified in the Car-Parrinello convergence scheme, which we termed TYPE I-III. At an exchange mixing factor of 0.15 we performed a series of simulations for different sets of projector functions and different $\vec{k}$-point grids to identify the true ground state. While the TYPE I state cannot be the ground state because it is found at a higher total energy, the total energies of the TYPE II and TYPE III states are identical within the accuracy of our simulations and both states are ground state candidates. We were not successful in addressing both the TYPE II state and the TYPE III state for all tested sets of projector functions. The potential landscape in the vicinity of the minima appears to be rather flat and in these cases a change in the set of projector functions or even the $\vec{k}$-point grid likely lifts one of the two minima which correspond to the TYPE II or Type III state.

The DOS of a TYPE II state in the majority spin direction possesses a peak of two occupied states well below the Fermi level, while the unoccupied states are situated in a peak at less than $2 \mathrm{eV}$ above the Fermi energy. States in the minority spin direction are unoccupied. For the TyPE III state, the DOS in the majority spin direction takes a three-peak structure with a partially occupied $4 f$ state at the Fermi level. While the peak separation of the fully occupied states and the completely unoccupied orbitals matches that of the TYPE II state, at an exchange mixing of 0.15 both peaks lie at $\sim 0.3 \mathrm{eV}$ higher energy.

A TyPE II state is found in simulations of $\operatorname{PrB}_{6}$ with an exchange mixing factor of 0.225 , which possesses two occupied $4 f$ orbitals at an energy of $E_{\mathrm{F}}-5 \mathrm{eV}$ in agreement with photoemission data [30]. Moreover, the energy distance between the occupied and unoccupied $4 f$ states is $5.9 \mathrm{eV}$ which is within the expected energy range above $5 \mathrm{eV}$.

For $\mathrm{NdB}_{6}$, we find a single ground state candidate, which like the TyPE III state of $\operatorname{PrB}_{6}$ has a fractionally occupied state pinned to the Fermi energy. When increasing the exchange mixing factor, only a small shift of the fully occupied states to lower energies and a larger upward shift of the unoccupied $4 f$ states occurred, which is in contrast to the simulations of $\mathrm{CeB}_{6}$ and also $\mathrm{PrB}_{6}$. This behavior of the $4 f$-projected DOS does not allow to shift the occupied $4 f$ states below $E_{\mathrm{F}}-2.5 \mathrm{eV}$ by increasing the exchange mixing factor, which is an energy far above the $4 f$ energies of $E_{\mathrm{F}}-7 \mathrm{eV}$ or $E_{\mathrm{F}}-6.25 \mathrm{eV}$ found in photoemission experiments [30, 141].

We suggest that the TyPE III DOSs of $\mathrm{PrB}_{6}$ and the DOSs of $\mathrm{NdB}_{6}$ from our DFT simulations with the PBE0r hybrid functional do not correctly represent 
the energy levels of the atomic orbitals within the respective materials. The reason for this is a systematic error that arises from the interpretation of the Kohn-Sham energies as the energy levels of atomic orbitals within the material. With this interpretation, the splitting of the $4 f$ levels due to the exchange mixing in the PBE0r hybrid functional does not lead to the correct spectrum in the case of a fractional occupancy of the $4 f$ states. This rationale is discussed in more detail in chapter 5 , where it is investigated on the basis of a simple model system. Conveniently, the model system also offers an explanation for the dependence of the $f$-state energies on the exchange mixing factor. Moreover, the model calculation clarifies the relation of the effective interaction within the $4 f$ shell and the distances between the $4 f$ peaks in the DOS. 


\section{The density of states of localized or- bitals with fractional occupancy: study of a model system}

In chapter 4 the results of DFT bulk simulations of the rare-earth hexaborides $\mathrm{CeB}_{6}, \mathrm{PrB}_{6}$, and $\mathrm{NdB}_{6}$ with the Perdew-Burke-Ernzerhof (PBE) functional and the PBE0r hybrid functional for multiple values of the exchange mixing factor are presented. With the PBE functional, the $4 f$-projected DOSs in the majority spin direction all have a single large feature, which is cut by the Fermi level. Hence, in agreement with previous studies, for these hexaborides the $4 f$ shell is shown to have a partial occupancy. The partial occupancy increases as we increase the atomic number of the lanthanide.

One major flaw of functionals within the local density approximation is an incomplete compensation of the unphysical interaction of an electron with itself that is present in the Hartree interaction. This produces a self-interaction error, which is especially large for rather localized orbitals like the $4 f$ orbitals, and results in an overestimation of the energies of occupied $4 f$ states. The PBE0r hybrid functional aims to correct the self-interaction error by mixing the exchange of the PBE functional with a portion of exact local exchange. Indeed, our results show that the PBE0r hybrid functional is in principle able to introduce a partial self-interaction correction as its application leads to a splitting of the $4 f$ levels, such that the fully occupied states are shifted to energies well below the Fermi energy.

However, our results with the PBE0r hybrid functional also indicate that the DFT DOS does not represent the correct excitation spectrum of the simulated material in the case of a fractional occupancy of the $4 f$ shell. A fractional occupancy of the $4 f$ shell is observed for converged states of our simulations of $\mathrm{PrB}_{6}$ and $\mathrm{NdB}_{6}$, see e.g. fig. 4.11 (TyPe III), fig. 4.12, and fig. 4.16. The $4 f$ orbital-projected DOSs in these cases possess a three-peak shape in the majority spin direction, with one fractionally occupied $4 f$ state at the Fermi level. In the minority spin direction the $4 f$ states remain unoccupied. Our results on $\mathrm{NdB}_{6}$ clearly show that the DOS does not agree with experimental photoemission spectra as it is not possible to shift the fully occupied $4 f$ states to the experimentally found energy by varying the exchange mixing within the PBE0r hybrid functional. We suggest that the main reason for the mismatch between our DOSs and experimental photoemission spectra lies in the way the Kohn-Sham energies depend on the fractional occupancy of the localized $4 f$ orbitals. In the following, we briefly outline the origin of a possibly fractional occupancy of the localized orbitals and the way the occupancies enter the Kohn-Sham energies. 
5 The density of states of localized orbitals with fractional occupancy: study of a model system

In a metallic material in which localized states hybridize with valence and conduction states, the localized states constitute an open subsystem. In general the localized states have to be described in terms of a mixed state and the occupancies of the localized orbitals can be fractional. We encounter such a situation in our DFT simulations of $\operatorname{PrB}_{6}$ and $\mathrm{NdB}_{6}$, where we have localized $4 f$ orbitals that hybridize with the metallic states of the crystal. The occupancies of the localized orbitals enter the optimization scheme of the Kohn-Sham wave functions via the PBE0r hybrid functional.

Within the PBE0r hybrid functional the exchange contribution, eq. (3.148), depends on the one-particle density matrix and thus on the occupancies of the localized orbitals. When the set of Kohn-Sham states and their occupancies are projected onto the localized orbitals within the PBE0r hybrid functional, fractional occupancies of the localized orbitals can occur. Further, Janak's theorem, eq. (3.132), says that the derivative of the total energy functional with respect to the occupancy of a Kohn-Sham state equals the energy of the corresponding Kohn-Sham state, $\partial E / \partial f_{n}=\varepsilon_{n}$, irrespective of the precise form of the exchange-correlation energy functional [74]. Using the PBE0r hybrid functional as exchange-correlation functional the Kohn-Sham energies receive contributions from the exact exchange term and the double-counting correction in addition to the contribution of the PBE functional. Importantly, according to eqs. (3.148) - (3.153), the contribution from the exact exchange to the Kohn-Sham energy reads (without the exchange mixing factor)

$$
\begin{aligned}
\Delta \varepsilon_{n}^{\mathrm{HF}, \mathrm{x}} & =\frac{\partial E_{\mathrm{x}}^{\mathrm{HF}}}{\partial f_{n}}=\sum_{A} \frac{\partial E_{\mathrm{x}, A}^{\mathrm{HF}}}{\partial f_{n}} \\
& =\sum_{A} \sum_{a b \in A}\left\langle\psi_{n} \mid \pi_{A a}\right\rangle\left[-\sum_{c d \in A} U_{A, d a b c} \rho_{A, c d}\right]\left\langle\pi_{A b} \mid \psi_{n}\right\rangle
\end{aligned}
$$

in the notation of section 3.9.6. Equation (5.2) depends on the one-particle density matrix and, hence, on the possibly fractional occupancies of the orbitals of each site $A$.

In the preceding paragraph we pointed out that a dependence of the KohnSham energy levels on the one-particle density matrix of the localized orbitals comes from the exchange energy term, eq. (3.148), in the PBE0r hybrid functional. The main aim of this chapter is to investigate the impact of the exchange admixture onto the Kohn-Sham energies and the DFT DOS. However, besides the exchange term, in DFT we have several further contributions to the effective potential of the Kohn-Sham system which render the calculation of the Kohn-Sham states and related energies rather complicated. A simpler case in which an analogous exchange term enters the calculation of energy levels of a non-interacting system is the Hartree-Fock approximation to a system of localized orbitals. Again, the localized orbitals should constitute an open system to allow for fractional occupancies.

In this chapter we study a model system in the Hartree-Fock approximation that is taken to be a simple analog to the Kohn-Sham system of DFT. Based on the model calculation we point out that the Hartree-Fock approximation to 
an interacting system of localized orbitals also leads to a spectral function of the effective non-interacting system that does not agree with the one-particle excitation spectrum of the original interacting system.

Our discussion is based on a simple model of an impurity with localized orbitals, which can host interacting electrons, and which is embedded in a noninteracting bath. The bath system and the impurity are allowed to exchange particles. The total number of electrons for the combined system is constant. We give a detailed description of the model system in the next section of this chapter.

For ease of understanding, we start with a spinless model system which is later extended to a spin-resolved version. The following analysis is performed for both versions of the model for a variety of model parameters. We calculate the minimum of the total energy, the corresponding ensemble of the impurity, and the one-particle spectral function of the localized orbitals. Further, in a second calculation we treat the impurity in the Hartree-Fock approximation. In the Hartree-Fock approach to the model system we again calculate the minimum total energy, the corresponding ensemble of the impurity, and the one-particle spectral function of the localized orbitals. We point out that the energy levels of the one-particle states of the effective non-interacting system of the HartreeFock approximation depend on the occupancies of the localized orbitals. From the one-particle energy levels we obtain the spectral function of the effective non-interacting Hartree-Fock system.

Having determined the spectral function in the many-body treatment and the spectral function in the Hartree-Fock approximation, we compare both quantities in a detailed discussion. Moreover, we use the results of the model calculation to rationalize the shape of the DOS of our DFT simulations of $\mathrm{CeB}_{6}, \mathrm{PrB}_{6}$, and $\mathrm{NdB}_{6}$ with the PBE0r hybrid functional.

\subsection{The model system}

For our model study we employ a model for an interacting atomic impurity embedded into a non-interacting bath. Between the impurity and the bath an exchange of particles is allowed. The bath and the impurity have the same Fermi level. The total number of electrons $N_{\text {tot }}$ within the model system is constant. In the following, we introduce the bath and the impurity in detail.

\subsubsection{The bath}

For the bath we take a large non-interacting system with a semi-infinite constant density of states $D \Theta(\varepsilon)$, where $\Theta(\varepsilon)$ denotes the Heaviside step function. The bath DOS's lower edge is fixed at zero energy, see fig. 5.1. Integrating the bath DOS up to the Fermi energy $E_{\mathrm{F}}$ yields the bath occupancy $N_{\text {bath }}$ :

$$
\int_{0}^{E_{\mathrm{F}}\left(N_{f}\right)} \mathrm{d} \varepsilon D=E_{\mathrm{F}}\left(N_{f}\right) D \stackrel{!}{=} N_{\text {bath }} \text {. }
$$


5 The density of states of localized orbitals with fractional occupancy: study of a model system

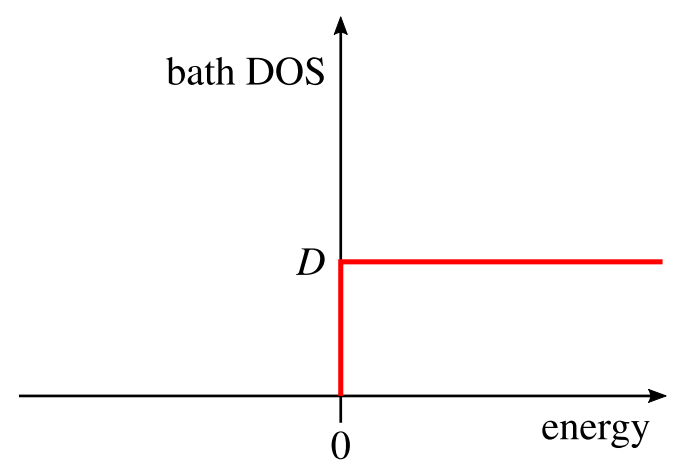

Figure 5.1: Sketch of the bath DOS.

In the combined model system the occupancy of the bath $N_{\text {bath }}$ and the total occupancy of the localized orbitals $N_{f}$ are coupled via

$$
N_{\text {tot }}=N_{\text {bath }}+N_{f}=\text { const, }
$$

where $N_{\text {tot }}$ is the total occupancy of the combined system. Hence, the Fermi energy is a function of $N_{f}$, with $N_{f} \leq N_{\text {tot }}$,

$$
E_{\mathrm{F}}\left(N_{f}\right)=\frac{N_{\text {bath }}}{D}=\frac{N_{\text {tot }}-N_{f}}{D} .
$$

The bath's total energy is

$$
E_{\text {bath }}\left(N_{f}\right)=\int_{0}^{E_{\mathrm{F}}\left(N_{f}\right)} \mathrm{d} \varepsilon D \varepsilon=\frac{1}{2} D E_{\mathrm{F}}^{2}\left(N_{f}\right)=\frac{1}{2 D}\left(N_{\mathrm{tot}}-N_{f}\right)^{2} .
$$

\subsubsection{The impurity}

The impurity of localized orbitals is modeled via a Hamiltonian of the form (3.22),

$$
\hat{H}_{\mathrm{loc}}=\sum_{a b} h_{a b} \hat{f}_{a}^{\dagger} \hat{f}_{b}+\frac{1}{2} \sum_{a b c d} U_{a b d c} \hat{f}_{a}^{\dagger} \hat{f}_{b}^{\dagger} \hat{f}_{c} \hat{f}_{d}
$$

where $h_{a b}$ is an element of the one-particle Hamiltonian matrix and $U_{a b c d}$ is an element of the interaction tensor. The operators $\hat{f}_{a}^{\dagger}$ and $\hat{f}_{a}$ create and annihilate a particle in a localized orbital $a$, respectively. We introduce two major simplifications:

1. The matrix elements of the non-interacting Hamiltonian shall have the form

$$
h_{a b}=\varepsilon_{f} \delta_{a b},
$$

i. e. a particle receives the same non-interacting energy contribution in either of the localized orbitals. 
2. We choose a simple interaction with interaction tensor elements

$$
U_{a b c d}=U \delta_{a c} \delta_{b d}, \quad \text { where } U_{a b a b}=U
$$

Hence, we have the same Hartree interaction between each two of the localized orbitals. Moreover, eq. (5.9) implies that the exchange is reduced to a contribution which compensates the self-interaction in the Hartree term.

With these simplifications, the impurity Hamiltonian (5.7) becomes

$$
\hat{H}_{\mathrm{loc}}=\varepsilon_{f} \sum_{a} \hat{f}_{a}^{\dagger} \hat{f}_{a}+\frac{U}{2} \sum_{a b} \hat{f}_{a}^{\dagger} \hat{f}_{b}^{\dagger} \hat{f}_{b} \hat{f}_{a} .
$$

We exploit the fermionic anticommutation relations $\left\{\hat{f}_{a}^{\dagger}, \hat{f}_{b}\right\}=\delta_{a b}$ and $\left\{\hat{f}_{a}, \hat{f}_{b}\right\}$ $=0$, so that the Hamiltonian (5.10) can be rewritten in terms of the total particle number operator

$$
\hat{N}_{f}=\sum_{a} \hat{n}_{a}
$$

The occupancy number operator for the orbital $a$ is denoted $\hat{n}_{a}=\hat{f}_{a}^{\dagger} \hat{f}_{a}$. This leads to

$$
\begin{aligned}
\hat{H}_{\mathrm{loc}} & =\varepsilon_{f} \sum_{a} \hat{n}_{a}+\frac{U}{2} \sum_{a b} \hat{n}_{a} \hat{n}_{b}-\frac{U}{2} \sum_{a} \hat{n}_{a} \\
& =\varepsilon_{f} \hat{N}_{f}+\frac{U}{2} \hat{N}_{f}^{2}-\frac{U}{2} \hat{N}_{f} .
\end{aligned}
$$

The Hamiltonian (5.13) is diagonal in the occupancy number basis. Hence, the basis can be chosen such that each eigenstate is a single Slater-determinant. The corresponding energy eigenvalues read

$$
\zeta_{\bar{N}_{f}}=\varepsilon_{f} \bar{N}_{f}+\frac{U}{2} \bar{N}_{f}^{2}-\frac{U}{2} \bar{N}_{f}
$$

Here and in the remainder of this chapter we use the overhead bar to indicate that $\bar{N}_{f} \in \mathbb{N}$ is an integer particle number. Clearly, all eigenstates with the same particle number $\bar{N}_{f}$ are degenerate. Let us also state the difference of the eigenvalues of two adjacent integer occupancies,

$$
\zeta_{\bar{N}_{f}+1}-\zeta_{\bar{N}_{f}}=\varepsilon_{f}+U \bar{N}_{f}
$$

as it will be reoccurring in the remainder of this chapter.

\section{2 | Many-body approach to the model}

Our model allows for a particle exchange between the bath and the impurity. A state of the impurity in general is an ensemble of many-particle states and a fractional occupancy of the impurity can occur. In this section we compute 
5 The density of states of localized orbitals with fractional occupancy: study of a model system

the ground state energy and the impurity spectral function for the ground state ensemble of the impurity in a many-particle approach.

The total energy of our model system is composed of the total energy of the bath and the total energy of the impurity and has the form

$$
E_{\mathrm{tot}}=E_{\mathrm{bath}}+E_{\mathrm{loc}} \text {. }
$$

The bath energy obeys eq. (5.6) and depends on the occupancy $N_{f}$ of the impurity. Moreover, the total energy of the impurity in general can be written as the expectation value of the Hamiltonian $\hat{H}_{\text {loc }}$ over an ensemble of manybody wave functions. We represent the ensemble of the impurity in terms of Slater determinants in the occupancy number basis of the localized orbitals and associated weights. This is a natural choice, because Slater determinants in the occupancy number basis of the localized orbitals are eigenstates to the Hamiltonian $\hat{H}_{\text {loc }}$, eq. (5.13), and form a basis of the Fock space of the impurity. For the calculations in the upcoming sections we introduce the notation $\left|\Phi_{M_{f}, i}\right\rangle$ for a Slater determinant of the ensemble, where the index $M_{f}$ is a label for the number of particles in the Slater determinant and $i$ is a label to distinguish different Slater determinants with the same particle number. The associated ensemble weights $w_{M_{f}, i}$ are labeled analogously.

The total energy of the localized orbitals can be expressed in terms of an ensemble average of the energy eigenvalues of $\hat{H}_{\text {loc }}$,

$$
\begin{aligned}
E_{\mathrm{loc}}(\{w\}) & =\sum_{M_{f}=0}^{N_{\text {orb }}} \sum_{i=1}^{N_{\text {states }}\left(M_{f}\right)} w_{M_{f}, i}\langle\underbrace{\left\langle\Phi_{M_{f}, i}\left|\hat{H}_{\mathrm{loc}}\right| \Phi_{M_{f}, i}\right\rangle}_{\zeta_{M_{f}}} \\
& =\sum_{M_{f}=0}^{N_{\text {orb }}} \zeta_{M_{f}} \sum_{i=1}^{N_{\text {states }}\left(M_{f}\right)} w_{M_{f}, i} .
\end{aligned}
$$

$N_{\text {states }}\left(M_{f}\right)$ is the number of different states with occupancy number $M_{f}$, and $N_{\text {orb }}$ is the number of localized orbitals. In the second equality we insert the eigenvalue $\zeta_{M_{f}}$, eq. (5.14). Note, that the eigenvalue $\zeta_{M_{f}}$ does only depend on the particle number index, but not on the individual state $i$ within the particle number sector.

To find the ground state total energy of the model system we proceed as follows. First, we minimize the total energy $E_{\text {loc }}(\{w\})$ of the impurity over the set of ensemble weights for a given occupancy $N_{f}$. In this way, we obtain the minimum total energy of the impurity $E_{\text {loc,min }}\left(N_{f}\right)$ as a function of $N_{f}$. We then minimize the total energy of the complete model system over $N_{f}$ to find the ground state energy and the ground state occupancy $N_{f}^{\mathrm{GS}}$. In the process of this minimization procedure we also determine the ensemble for the impurity, which minimizes the total energy $E_{\text {tot }}$. From this ensemble we obtain the spectral function for the localized orbitals. 


\subsubsection{The ground state total energy of the model}

The eigenvalues of $\hat{H}_{\text {loc }}$ follow a convex parabola, eq. (5.14), when moving across different particle number sectors. Hence, the minimum energy for an integer total occupancy $N_{f}=\bar{N}_{f}$ of the localized orbitals can only be obtained if the only non-zero ensemble weights are in the sector of particle number $\bar{N}_{f}$. I. e. for $M_{f} \neq \bar{N}_{f}$ we have ensemble weights $w_{M_{f}, i}=0$ for all $i$, and for $M_{f}=\bar{N}_{f}$ there is at least one $i$ for which $w_{M_{f}, i}>0$. Hence,

$$
E_{\mathrm{loc}, \min }\left(\left\{w_{\bar{N}_{f}}\right\}, \bar{N}_{f}\right)=\zeta_{\bar{N}_{f}} \sum_{i}^{N_{\text {states }}\left(\bar{N}_{f}\right)} w_{\bar{N}_{f}, i}=\zeta_{\bar{N}_{f}},
$$

where we exploited that $\sum_{i}^{N_{\text {states }}\left(\bar{N}_{f}\right)} w_{\bar{N}_{f}, i}=1$.

Before we continue with the case of a non-integer total occupancy number, we define the floor function $\lfloor\cdot\rfloor$ and the ceiling function $\lceil\cdot\rceil$. For a fractional number $x,\lfloor x\rfloor$ returns the closest integer number smaller than $x$ and $\lceil x\rceil$ returns the closest integer number larger than $x$.

In the case of a non-integer total occupancy $N_{f}$ of the impurity, again, because the parabola (5.14) is a convex function, the energy is minimized by an ensemble of Slater determinants in which only the Slater determinants with integer particle numbers $\left\lfloor N_{f}\right\rfloor$ and $\left\lceil N_{f}\right\rceil$ have non-zero weights. I. e. for $M_{f} \neq\left\lfloor N_{f}\right\rfloor$ and $M_{f} \neq\left\lceil N_{f}\right\rceil$ we have $w_{M_{f}, i}=0$ for all $i$. Moreover, for $M_{f}=\left\lfloor N_{f}\right\rfloor$ there is at least one $i$ for which $w_{\left\lfloor N_{f}\right\rfloor, i}>0$ and also for $M_{f}=\left\lceil N_{f}\right\rceil$ there is at least one $i$ for which $w_{\left\lceil N_{f}\right\rceil, i}>0$. Consequently we find

$$
\begin{aligned}
E_{\mathrm{loc}, \min }\left(\left\{w_{\left\lfloor N_{f}\right\rfloor}\right\},\left\{w_{\left\lceil N_{f}\right\rceil}\right\}, N_{f}\right) & =\zeta_{\left\lfloor N_{f}\right\rfloor}^{N_{\text {states }}\left(\left\lfloor N_{f}\right\rfloor\right)} \sum_{i} w_{\left\lfloor N_{f}\right\rfloor, i}+\zeta_{\left\lceil N_{f}\right\rceil} \sum_{i}^{N_{\text {states }}\left(\left\lceil N_{f}\right\rceil\right)} w_{\left\lceil N_{f}\right\rceil, i} \\
& =\zeta_{\left\lfloor N_{f}\right\rfloor}+\left[\sum_{i}^{N_{\text {states }}\left(\left\lceil N_{f}\right\rceil\right)} w_{\left\lceil N_{f}\right\rceil, i}\right]\left(\zeta_{\left\lceil N_{f}\right\rceil}-\zeta_{\left\lfloor N_{f}\right\rfloor}\right)
\end{aligned}
$$

where we exploited

$$
\sum_{i}^{N_{\text {states }}\left(\left\lfloor N_{f}\right\rfloor\right)} w_{\left\lfloor N_{f}\right\rfloor, i}+\sum_{i}^{N_{\text {states }}\left(\left\lceil N_{f}\right\rceil\right)} w_{\left\lceil N_{f}\right\rceil, i}=1 .
$$

Hence, the energy minimum for a fractional occupancy $N_{f}=\left\lfloor N_{f}\right\rfloor+n_{f}^{\text {frac }}$ lies on the straight connection line between the coordinates $\left(\left\lfloor N_{f}\right\rfloor, \zeta_{\left\lfloor N_{f}\right\rfloor}\right)$ and $\left(\left\lceil N_{f}\right\rceil, \zeta_{\left\lceil N_{f}\right\rceil}\right)$ in the $E_{\mathrm{loc}}\left(N_{f}\right)$ diagram. Using the intercept theorem we obtain (see fig. 5.2)

$$
\sum_{i}^{N_{\text {states }}\left(\left\lceil N_{f}\right\rceil\right)} w_{\left\lceil N_{f}\right\rceil, i}=n_{f}^{\mathrm{frac}}
$$


5 The density of states of localized orbitals with fractional occupancy: study of a model system

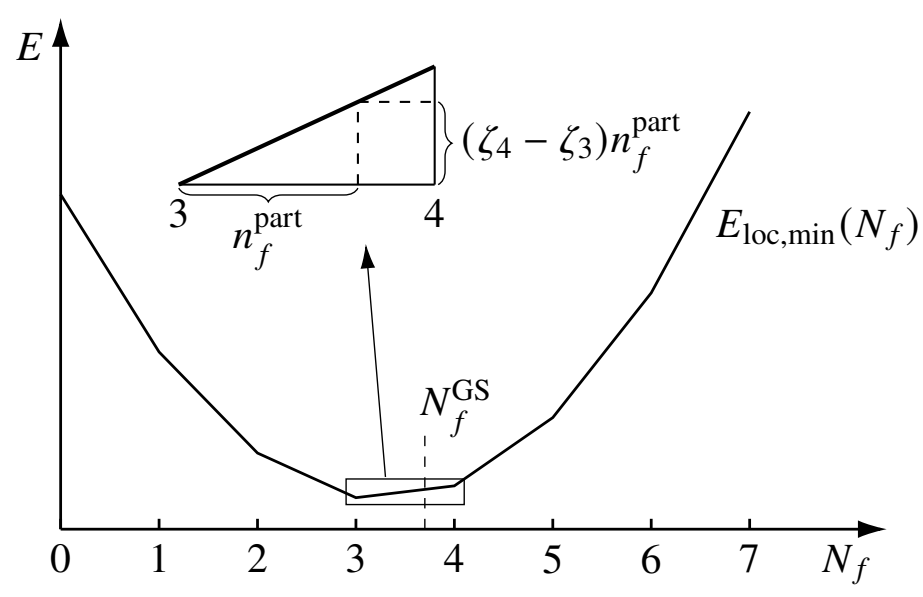

Figure 5.2: Schematic diagram of an example curve $E_{\text {loc,min }}\left(N_{f}\right)$, and triangle for the slope between $N_{f}=3$, and $N_{f}=4$. For a fractional occupancy of $N_{f}^{\mathrm{GS}}=3.7$, the fraction $n_{f}^{\text {part }}=0.7$ of the occupancy results in the contribution $\left(\zeta_{4}-\zeta_{3}\right) n_{f}^{\text {part }}$ to the energy.

and

$$
\sum_{i}^{N_{\text {states }}\left(\left\lfloor N_{f}\right\rfloor\right)} w_{\left\lfloor N_{f}\right\rfloor, i}=1-n_{f}^{\mathrm{frac}} .
$$

In conclusion, the minimum total energy for the impurity for a fractional occupancy $N_{f}$ is (see also [90])

$$
\begin{aligned}
E_{\mathrm{loc}, \min }\left(N_{f}\right) & =\left(1-n_{f}^{\mathrm{frac}}\right) \zeta_{\left\lfloor N_{f}\right\rfloor}+n_{f}^{\mathrm{frac}} \zeta_{\left\lceil N_{f}\right\rceil} \\
& =\zeta_{\left\lfloor N_{f}\right\rfloor}+n_{f}^{\mathrm{frac}} \cdot\left[\zeta_{\left\lceil N_{f}\right\rceil}-\zeta_{\left\lfloor N_{f}\right\rfloor}\right] \\
& =\varepsilon_{f} N_{f}+\frac{U}{2} N_{f}^{2}-\frac{U}{2}\left\lfloor N_{f}\right\rfloor-\frac{U}{2}\left(n_{f}^{\mathrm{frac}}\right)^{2} .
\end{aligned}
$$

For the full model, consisting of the bath and the localized orbitals, the ground state energy $E_{\mathrm{tot}}^{\mathrm{GS}}$ can be found by minimizing the total energy

$$
\begin{aligned}
E_{\mathrm{tot}}\left(N_{f}\right) & =E_{\mathrm{bath}}\left(N_{f}\right)+E_{\mathrm{loc}, \text { min }}\left(N_{f}\right) \\
& =\frac{1}{2 D}\left(N_{\mathrm{tot}}-\left\lfloor N_{f}\right\rfloor-n_{f}^{\mathrm{frac}}\right)^{2}+\zeta_{\left\lfloor N_{f}\right\rfloor}+n_{f}^{\mathrm{frac}} \cdot\left[\zeta_{\left\lceil N_{f}\right\rceil}-\zeta_{\left\lfloor N_{f}\right\rfloor}\right]
\end{aligned}
$$

with respect to the total occupancy $N_{f}$ of the localized orbitals:

$$
E_{\mathrm{tot}}^{\mathrm{GS}}=\min _{N_{f}} E_{\mathrm{tot}}\left(N_{f}\right)
$$

Note, that the total energy is generally not differentiable with respect to $N_{f}$ at integer $N_{f}=\bar{N}_{f}$. This is because $E_{\text {loc,min }}\left(N_{f}\right)$ is a set of linear curves, matched in value at $\bar{N}_{f}$, where generally a derivative discontinuity occurs at $\bar{N}_{f}$ due to different slopes of the adjacent linear segments (see fig. 5.2). However, for 
fractional occupancies we find

$$
\frac{\partial E_{\mathrm{tot}}}{\partial n_{f}^{\text {frac }}}=-\frac{1}{D}\left(N_{\mathrm{tot}}-\left\lfloor N_{f}\right\rfloor-n_{f}^{\mathrm{frac}}\right)+\zeta_{\left\lceil N_{f}\right\rceil}-\zeta_{\left\lfloor N_{f}\right\rfloor} .
$$

Inserting eq. (5.5) into eq. (5.31) and exploiting the minimum condition $\partial E_{\text {tot }} / \partial n_{f}^{\text {frac }}=0$ yields the important result that the Fermi energy is the difference in the energy eigenvalues for adjacent integer particle numbers,

$$
\begin{aligned}
E_{\mathrm{F}} & \left.=\zeta_{\left\lceil N_{f}\right\rceil}-\zeta_{\left\lfloor N_{f}\right\rfloor}\right\rfloor \\
& =\varepsilon_{f}+U\left\lfloor N_{f}\right\rfloor .
\end{aligned}
$$

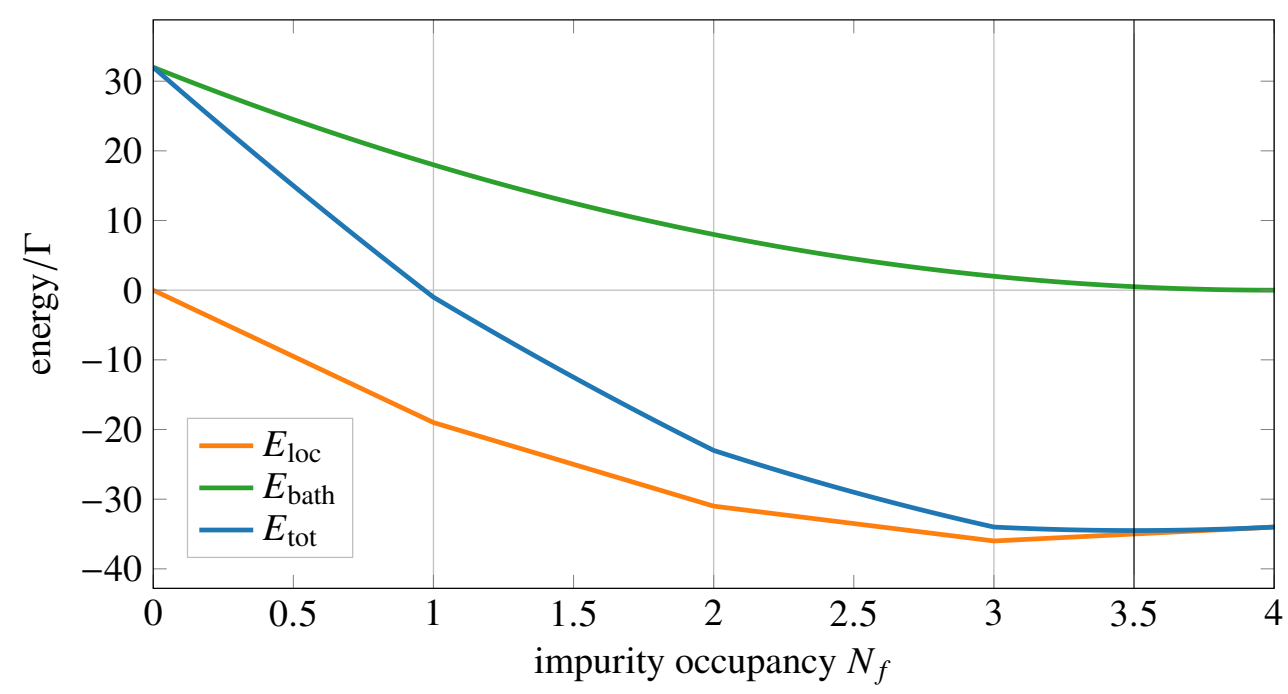

Figure 5.3: Example energy curves of the bath, of the localized orbitals, and of the total system versus the total occupancy number $N_{f}$ of the localized orbitals. The black vertical line at $N_{f}=3.5$ marks the minimum of $E_{\text {tot }}$. Model parameters are $\varepsilon_{f} / \Gamma=-19, U / \Gamma=7$, $D \Gamma=1 / 4, N_{\text {tot }}=4$.

Parameters for the model system are derived from the corresponding quantities within the rare-earth hexaborides. The interaction parameter $U$ of the rare-earth hexaboride $4 f$ orbitals is $U_{\mathrm{La}}=5 \mathrm{eV}$ for $\mathrm{LaB}_{6}$ [137] and tends to increase when moving further through the rare earth hexaboride series [133]. We define an energy scale $\Gamma=1 \mathrm{eV}$ and choose an interaction strength of $U=7 \Gamma$. For the bath DOS we set $D=1 / L(\mathrm{~B} \mathrm{p})=1 /(4 \Gamma)$, where $L(\mathrm{~B} \mathrm{p})=4 \Gamma$ is the approximate energy range for the projected DOS of the boron $p$ states of the light rare-earth hexaborides in the vicinity of the Fermi level to host a total of one electron. The non-interacting energy level $\varepsilon_{f}$ of the localized orbitals is varied to adjust the occupancy numbers of the localized orbitals and the bath.

As an example the energy curves of $E_{\text {tot }}\left(N_{f}\right), E_{\text {bath }}\left(N_{f}\right)$, and $E_{\mathrm{loc}}\left(N_{f}\right)$ are plotted in fig. 5.3 for a selected set of model parameters. We show a case for which the energy eigenvalues of two neighboring particle sectors of the impurity are not degenerate. Then $E_{\text {loc }}$ can have a minimum only at integer 
5 The density of states of localized orbitals with fractional occupancy: study of a model system

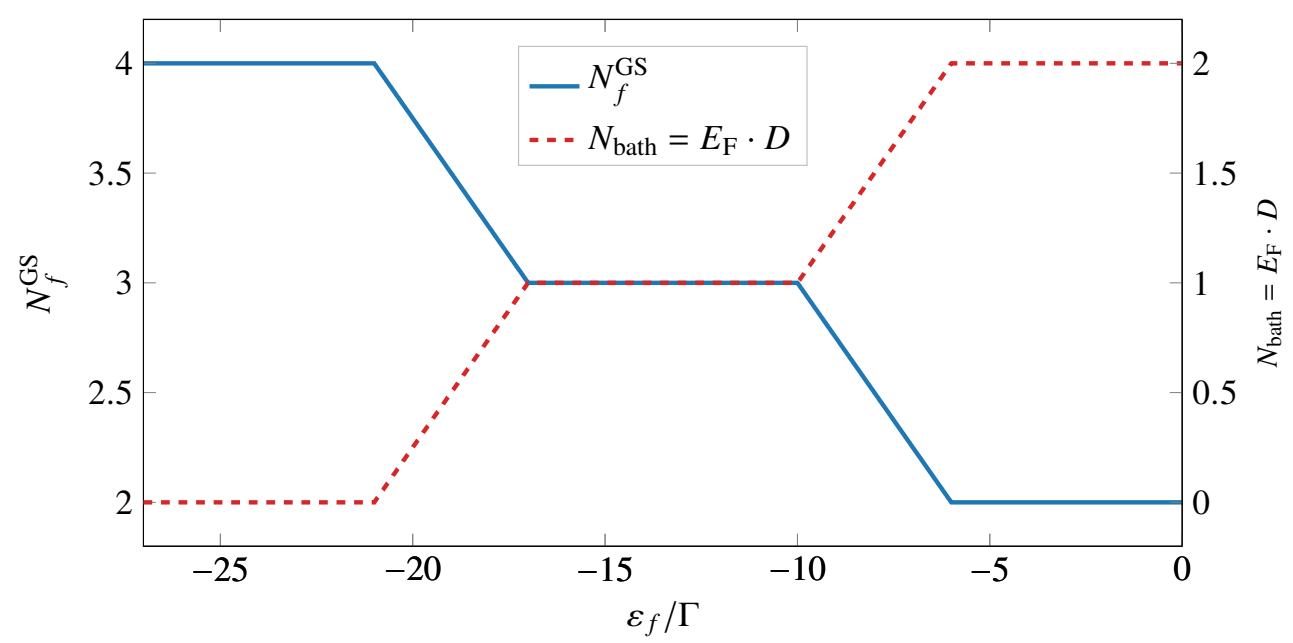

Figure 5.4: Dependence of the total ground state occupancy of the localized orbitals $N_{f}^{\mathrm{GS}}$ and the bath occupancy $N_{\text {bath }}$ on the energy parameter of the localized orbitals $\varepsilon_{f}$. Model parameters are $U / \Gamma=7, D \Gamma=$ $1 / 4, N_{\text {tot }}=4$.

values of $N_{f}$. However, the minimum of $E_{\text {tot }}$ can be at a fractional occupancy of the localized orbitals.

We remark that our model allows for the case of two neighboring particle sectors, $\bar{N}_{f}$ and $\bar{N}_{f}+1$, to have degenerate eigenvalues, if $\varepsilon_{f}=-U \bar{N}_{f}$. In that case $E_{\text {loc }}$ is minimal along the flat line between the integer particle numbers for which the same value of $E_{\mathrm{loc}}$ is obtained. Then, the minimum of $E_{\text {tot }}$ is at the larger of the two integer particle numbers or beyond because of the strict monotonic decrease of $E_{\text {bath }}\left(N_{f}\right)$. More than two particle sectors having the same energy are not possible since with $\varepsilon_{f}=-U \bar{N}_{f}$, from eq. (5.15) we obtain $\zeta_{\bar{N}_{f}}=\zeta_{\bar{N}_{f}-1}-U$ and $\zeta_{\bar{N}_{f}+2}=\zeta_{\bar{N}_{f}+1}+U$.

Due to the kinks of the total energy curve at integer occupancies an integer ground state occupancy is not unique to a single value of $\varepsilon_{f}$. Figure 5.4 shows a plot of the dependence of the ground state occupancy number $N_{f}^{\mathrm{GS}}$ on the model parameter $\varepsilon_{f}$. As $\varepsilon_{f}$ is varied, integer occupancies extend over broad plateaus. However, when the value of $\varepsilon_{f}$ results in a fractional occupancy of the impurity, each value of $\varepsilon_{f}$ leads to a unique value of $N_{f}^{\mathrm{GS}}$.

\subsubsection{The spectral function}

With many experimental methods, including photoemission spectroscopy and inverse photoemission spectroscopy, the one-particle excitation spectrum of a material is measured. For a comparison with theoretical data often the DOS of the Kohn-Sham system from DFT is used as a reference. In the context of our model approach we aim at a comparison of the single-particle excitation spectra of the localized orbitals, obtained from the many-body approach and in the Hartree-Fock approximation. Moreover, the excitation spectra of the localized orbitals of the model system are compared to the $4 f$-projected DOS 
of our DFT simulations of the rare-earth hexaborides.

In many-particle theory the single-particle excitation spectrum is represented by the single-particle spectral function

$$
A(a, \varepsilon)=\sum_{n, m}\left(w_{n}+w_{m}\right)\left|\left\langle\Psi_{n}\left|\hat{f}_{a}\right| \Psi_{m}\right\rangle\right|^{2} \delta\left(\varepsilon+\zeta_{n}-\zeta_{m}\right),
$$

where $\left|\Psi_{n}\right\rangle$ and $\left|\Psi_{m}\right\rangle$ are many-body states within an ensemble. $\left|\Psi_{n}\right\rangle$ and $\left|\Psi_{m}\right\rangle$ occur with probabilities $w_{n}$ and $w_{m}$ and are associated with the energy eigenvalues $\zeta_{n}$ and $\zeta_{m}$, respectively. $\hat{f}_{a}$ annihilates a particle of quantum number $a$.

On the one hand, the spectral function gives the probability that a particle in an initial quantum state $a$ is measured with an energy $\varepsilon$ when removed from the system. On the other hand it also is a measure for the probability that a particle will be found in a quantum state $a$ after an excitation of the system by adding a particle of energy $\varepsilon$. The spectral function can be viewed as a generalization of the DOS that covers non-interacting as well as interacting particle systems. In the following we calculate the spectral function for the localized states of the model system. It is compared to the spectral function of the model system in Hartree-Fock approximation in section 5.4.

In our model the non-interacting part of the Hamiltonian gives the same contribution $\varepsilon_{f}$ for each of the localized orbitals and between each two of the localized orbitals we have the same interaction $U$. Hence, there is a plethora of many-particle mixed states with the same ground state energy. This means that the ground-state spectral function for a localized orbital $a$ depends on the choice of the ensemble weights. However, the average ground-state spectral function over all localized orbitals is independent of this choice. Furthermore, this average spectral function is equal to the spectral function of a single orbital in the case where for each integer particle sector all weights are equal, i. e. $w_{M_{f}, m}=w_{M_{f}, n}$ for all $M_{f}$. In statistical physics such a mixed state is referred to as a state of maximum ignorance or maximum entropy, see e. $\mathrm{g}$. [144-148], because all orbitals are treated on equal footing and no orbital is treated preferentially. A thermal ensemble satisfies the principle of maximum entropy. We formally work in the zero-temperature limit $T \rightarrow 0$ and discard the ensembles in which the principle of maximum entropy is violated.

Following the above arguments, instead of calculating the spectral function of a single localized orbital $a$, we calculate the average spectral function over all localized orbitals. For a ground state ensemble given by the Slater determinants $\left|\Phi_{M_{f}, i}\right\rangle$ with the associated weights $w_{M_{f}, i}$, the spectral function of the impurity for an occupancy number $N_{f}$ reads

$$
\begin{aligned}
A_{\text {loc }}\left(\varepsilon, N_{f}\right)= & \frac{1}{N_{\text {orb }}} \sum_{a} \sum_{M_{f}=0}^{N_{\text {orb }}} \sum_{m=1}^{N_{\text {states }}\left(M_{f}\right)} \sum_{Q_{f}=0}^{N_{\text {orb }}} \sum_{q=1}^{N_{\text {states }}\left(Q_{f}\right)}\left(w_{M_{f}, m}+w_{Q_{f}, q}\right) \\
& \times\left|\left\langle\Phi_{M_{f}, m}\left|\hat{f}_{a}\right| \Phi_{Q_{f}, q}\right\rangle\right|^{2} \delta\left(\zeta_{M_{f}, m}-\zeta_{Q_{f}, q}+\varepsilon\right)
\end{aligned}
$$


5 The density of states of localized orbitals with fractional occupancy: study of a model system

$$
\begin{aligned}
& =\frac{1}{N_{\mathrm{orb}}} \sum_{a} \sum_{M_{f}=0}^{N_{\mathrm{orb}}-1} \sum_{m=1}^{N_{\text {states }}\left(M_{f}\right)} \sum_{q=1}^{N_{\text {states }}\left(M_{f}+1\right)}\left(w_{M_{f}, m}+w_{M_{f}+1, q}\right) \\
& \quad \times\left|\left\langle\Phi_{M_{f}, m}\left|\hat{f}_{a}\right| \Phi_{M_{f}+1, q}\right\rangle\right|^{2} \delta\left(\zeta_{M_{f}}-\zeta_{M_{f}+1}+\varepsilon\right)
\end{aligned}
$$

In the first equality the Slater determinants are grouped according to their particle number sectors, which we label $M_{f}$ and $Q_{f}$, respectively. Furthermore, within each particle number sector we use the indices $m$, and $q$ to distinguish between different states. It is then possible to eliminate the sum over $Q_{f}$ since the matrix element $\left|\left\langle\Phi_{M_{f}, m}\left|\hat{f}_{a}\right| \Phi_{Q_{f}, q}\right\rangle\right|^{2}$ can be non-zero only, if $Q_{f}=M_{f}+1$. In the following we discriminate between integer and non-integer occupancy. For brevity we omit the edge cases $N_{f}=0$ and $N_{f}=N_{\text {orb }}$, where $N_{\text {orb }}$ is the number of localized orbitals, since they are not needed for our discussion.

If the total occupancy of the localized orbitals is integer, $N_{f}=\bar{N}_{f}$, only the ensemble weights $\left\{w_{\bar{N}_{f}}\right\}$ are non-zero and the spectral function (5.36) becomes

$$
\begin{aligned}
& A_{\mathrm{loc}}\left(\varepsilon, \bar{N}_{f}\right)=\frac{1}{N_{\text {orb }}} \sum_{a} \sum_{m=1}^{N_{\text {states }}\left(\bar{N}_{f}-1\right)} \sum_{q=1}^{N_{\text {states }}\left(\bar{N}_{f}\right)} w_{\bar{N}_{f}, q} \\
& \times\left|\left\langle\Phi_{\bar{N}_{f}-1, m}\left|\hat{f}_{a}\right| \Phi_{\bar{N}_{f}, q}\right\rangle\right|^{2} \delta\left(\zeta_{\bar{N}_{f}-1}-\zeta_{\bar{N}_{f}}+\varepsilon\right) \\
& +\frac{1}{N_{\text {orb }}} \sum_{a} \sum_{m=1}^{N_{\text {states }}\left(\bar{N}_{f}\right)} \sum_{q=1}^{N_{\text {states }}\left(\bar{N}_{f}+1\right)} w_{\bar{N}_{f}, m} \\
& \times\left|\left\langle\Phi_{\bar{N}_{f}, m}\left|\hat{f}_{a}\right| \Phi_{\bar{N}_{f}+1, q}\right\rangle\right|^{2} \delta\left(\zeta_{\bar{N}_{f}}-\zeta_{\bar{N}_{f}+1}+\varepsilon\right) \\
& =\frac{1}{N_{\text {orb }}} \sum_{a} \sum_{m=1}^{N_{\text {states }}\left(\bar{N}_{f}-1\right)} \sum_{\substack{q^{\prime} \\
\left\langle\Phi_{\bar{N}_{f}, q^{\prime}} \hat{n}_{a} \mid \Phi_{\bar{N}_{f}, q^{\prime}}\right\rangle=1}} w_{\bar{N}_{f}, q^{\prime}} \\
& \times\left|\left\langle\Phi_{\bar{N}_{f}-1, m}\left|\hat{f}_{a}\right| \Phi_{\bar{N}_{f}, q^{\prime}}\right\rangle\right|^{2} \delta\left(\zeta_{\bar{N}_{f}-1}-\zeta_{\bar{N}_{f}}+\varepsilon\right) \\
& +\frac{1}{N_{\text {orb }}} \sum_{a} \sum_{\substack{m^{\prime} \\
\left\langle\Phi_{\bar{N}_{f}, m^{\prime}}\left|\hat{n}_{a}\right| \Phi_{\bar{N}_{f}, m^{\prime}}\right\rangle=0}} \sum_{q=1}^{N_{\text {states }}\left(\bar{N}_{f}+1\right)} w_{\bar{N}_{f}, m^{\prime}} \\
& \times\left|\left\langle\Phi_{\bar{N}_{f}, m^{\prime}}\left|\hat{f}_{a}\right| \Phi_{\bar{N}_{f}+1, q}\right\rangle\right|^{2} \delta\left(\zeta_{\bar{N}_{f}}-\zeta_{\bar{N}_{f}+1}+\varepsilon\right) \\
& =\frac{1}{N_{\mathrm{orb}}} \sum_{a} \sum_{\substack{q^{\prime} \\
\left\langle\Phi_{\bar{N}_{f}, q^{\prime}}\left|\hat{n}_{a}\right| \Phi_{\bar{N}_{f}, q^{\prime}}\right\rangle=1}} w_{\bar{N}_{f}, q^{\prime}} \delta\left(\zeta_{\bar{N}_{f}-1}-\zeta_{\bar{N}_{f}}+\varepsilon\right) \\
& +\frac{1}{N_{\mathrm{orb}}} \sum_{a} \sum_{\substack{m^{\prime} \\
\left\langle\Phi_{\bar{N}_{f}, m^{\prime}}\left|\hat{n}_{a}\right| \Phi_{\bar{N}_{f}, m^{\prime}}\right\rangle=0}} w_{\bar{N}_{f}, m^{\prime}} \delta\left(\zeta_{\bar{N}_{f}}-\zeta_{\bar{N}_{f}+1}+\varepsilon\right) .
\end{aligned}
$$

In the second equality, the sum over $q$ in the first part is reduced to a sum 
over the Slater determinants for which the orbital $a$ is occupied. This is because the matrix element $\left\langle\Phi_{\bar{N}_{f}-1, m}\left|\hat{f}_{a}\right| \Phi_{\bar{N}_{f}, q}\right\rangle$ vanishes otherwise. In the sum over $q^{\prime}$ only the ensemble weights remain for which the corresponding Slater determinant has an electron in the orbital $a$. Similarly, the sum over $m$ in the second part of eq. (5.38) is reduced to a sum over the Slater determinants for which the orbital $a$ is not occupied. To arrive at the third equality, the sum over $m$ in the first part and the sum over $q$ in the second part are executed. These select the only states for which the matrix elements $\left\langle\Phi_{\bar{N}_{f}-1, m}\left|\hat{f}_{a}\right| \Phi_{\bar{N}_{f}, q^{\prime}}\right\rangle$ and $\left\langle\Phi_{\bar{N}_{f}, m^{\prime}}\left|\hat{f}_{a}\right| \Phi_{\bar{N}_{f}+1, q}\right\rangle$, respectively, do not vanish.

The remaining double sum over $a$, and $q^{\prime}$ in the first part can be rewritten in terms of a single sum, where each ensemble weight for Slater determinants with occupancy $\bar{N}_{f}$ occurs $\bar{N}_{f}$ times. Similarly, in the second part we find a summation, where each ensemble weight for the Slater determinants with occupancy $\bar{N}_{f}$ occurs $\left(N_{\text {orb }}-\bar{N}_{f}\right)$ times. As a result the spectral function simplifies to

$$
\begin{aligned}
A\left(\varepsilon, \bar{N}_{f}\right)= & \frac{1}{N_{\text {orb }}}\left[\bar{N}_{f} \sum_{i}^{N_{\text {states }}\left(\bar{N}_{f}\right)} w_{\bar{N}_{f}, i}\right] \delta\left(\zeta_{\bar{N}_{f}-1}-\zeta_{\bar{N}_{f}}+\varepsilon\right) \\
& +\frac{1}{N_{\mathrm{orb}}}\left[\left(N_{\mathrm{orb}}-\bar{N}_{f}\right) \sum_{i}^{N_{\text {states }}\left(\bar{N}_{f}\right)} w_{\bar{N}_{f}, i}\right] \delta\left(\zeta_{\bar{N}_{f}}-\zeta_{\bar{N}_{f}+1}+\varepsilon\right) \\
= & \frac{\bar{N}_{f}}{N_{\mathrm{orb}}} \delta\left(\zeta_{\bar{N}_{f}-1}-\zeta_{\bar{N}_{f}}+\varepsilon\right)+\frac{N_{\mathrm{orb}}-\bar{N}_{f}}{N_{\mathrm{orb}}} \delta\left(\zeta_{\bar{N}_{f}}-\zeta_{\bar{N}_{f}+1}+\varepsilon\right),
\end{aligned}
$$

where it was exploited that the sum over all ensemble weights equals 1 . Thus, for an integer occupancy $\bar{N}_{f}$, according to eq. (5.15) the ground state spectral function has a total of two $\delta$-peaks at the energies

$$
\Delta \zeta_{-}=\zeta_{\bar{N}_{f}}-\zeta_{\bar{N}_{f}-1}=\varepsilon_{f}+U\left(\bar{N}_{f}-1\right)
$$

and

$$
\Delta \zeta_{+}=\zeta_{\bar{N}_{f}+1}-\zeta_{\bar{N}_{f}}=\varepsilon_{f}+U \bar{N}_{f}
$$

In the case of a fractional total occupancy $N_{f}$, all the sets of ensemble weights $\left\{w_{\left\lfloor N_{f}\right\rfloor, 1}, \ldots, w_{\left\lceil N_{f}\right\rceil, 1}, \ldots\right\}$ which lead to the targeted fractional occupancy can have non-zero weights. To find an expression for the spectral function, we proceed similarly to the case of an integer occupancy $\bar{N}_{f}$ :

$$
\begin{aligned}
A\left(\varepsilon, N_{f}\right)= & \frac{1}{N_{\text {orb }}} \sum_{a} \sum_{m=1}^{N_{\text {states }}\left(\left\lfloor N_{f}\right\rfloor-1\right)} \sum_{q=1}^{N_{\text {states }}\left(\left\lfloor N_{f}\right\rfloor\right)} w_{\left\lfloor N_{f}\right\rfloor, q} \\
& \times\left|\left\langle\Phi_{\left\lfloor N_{f}\right\rfloor-1, m}\left|\hat{f}_{a}\right| \Phi_{\left\lfloor N_{f}\right\rfloor, q}\right\rangle\right|^{2} \delta\left(\zeta_{\left\lfloor N_{f}\right\rfloor-1}-\zeta_{\left\lfloor N_{f}\right\rfloor}+\varepsilon\right)
\end{aligned}
$$


5 The density of states of localized orbitals with fractional occupancy: study of a model system

$$
\begin{aligned}
& +\frac{1}{N_{\text {orb }}} \sum_{a} \sum_{m=1}^{N_{\text {states }}\left(\left\lfloor N_{f}\right\rfloor\right)} \sum_{q=1}^{N_{\text {states }}\left(\left\lceil N_{f}\right\rceil\right)}\left(w_{\left\lfloor N_{f}\right\rfloor, m}+w_{\left\lceil N_{f}\right\rceil, q}\right) \\
& \times\left|\left\langle\Phi_{\left\lfloor N_{f}\right\rfloor, m}\left|\hat{f}_{a}\right| \Phi_{\left\lceil N_{f}\right\rceil, q}\right\rangle\right|^{2} \delta\left(\zeta_{\left\lfloor N_{f}\right\rfloor}-\zeta_{\left\lceil N_{f}\right\rceil}+\varepsilon\right) \\
& +\frac{1}{N_{\text {orb }}} \sum_{a} \sum_{m=1}^{N_{\text {states }}\left(\left\lceil N_{f}\right\rceil\right)} \sum_{q=1}^{N_{\text {states }}\left(\left\lceil N_{f}\right\rceil+1\right)} w_{\left\lceil N_{f}\right\rceil, m} \\
& \times\left|\left\langle\Phi_{\left\lceil N_{f}\right\rceil, m}\left|\hat{f}_{a}\right| \Phi_{\left\lceil N_{f}\right\rceil+1, q}\right\rangle\right|^{2} \delta\left(\zeta_{\left\lceil N_{f}\right\rceil}-\zeta_{\left\lceil N_{f}\right\rceil+1}+\varepsilon\right) \\
& =\frac{1}{N_{\text {orb }}} \sum_{a} \sum_{m=1}^{N_{\text {states }}\left(\left\lfloor N_{f}\right\rfloor-1\right)} \sum_{\substack{q^{\prime} \\
\left\langle\Phi_{\left\lfloor N_{f}\right\rfloor, q^{\prime}}\left|\hat{n}_{a}\right| \Phi_{\left\lfloor N_{f}\right\rfloor, q^{\prime}}\right\rangle=1}} w_{\left\lfloor N_{f}\right\rfloor, q^{\prime}} \\
& \times\left|\left\langle\Phi_{\left\lfloor N_{f}\right\rfloor-1, m}\left|\hat{f}_{a}\right| \Phi_{\left\lfloor N_{f}\right\rfloor, q^{\prime}}\right\rangle\right|^{2} \delta\left(\zeta_{\left\lfloor N_{f}\right\rfloor-1}-\zeta_{\left\lfloor N_{f}\right\rfloor}+\varepsilon\right) \\
& +\frac{1}{N_{\text {orb }}} \sum_{a} \sum_{\substack{m^{\prime} \\
\left\langle\Phi_{\left\lfloor N_{f}\right\rfloor, m^{\prime}}\left|\hat{n}_{a}\right| \Phi_{\left.\left\lfloor N_{f}\right\rfloor, m^{\prime}\right\rangle=0}\right.}} \sum_{q=1}^{N_{\text {states }}\left(\left\lceil N_{f}\right\rceil\right)} w_{\left\lfloor N_{f}\right\rfloor, m^{\prime}} \\
& \times\left|\left\langle\Phi_{\left\lfloor N_{f}\right\rfloor, m^{\prime}}\left|\hat{f}_{a}\right| \Phi_{\left\lceil N_{f}\right\rceil, q}\right\rangle\right|^{2} \delta\left(\zeta_{\left\lfloor N_{f}\right\rfloor}-\zeta_{\left\lceil N_{f}\right\rceil}+\varepsilon\right) \\
& +\frac{1}{N_{\text {orb }}} \sum_{a} \sum_{m=1}^{N_{\text {states }}\left(\left\lfloor N_{f}\right\rfloor\right)} \quad \sum_{q^{\prime}} \quad w_{\left\lceil N_{f}\right\rceil, q^{\prime}} \\
& \left\langle\Phi_{\left\lceil N_{f}\right\rceil, q^{\prime}}\left|\hat{n}_{a}\right| \Phi_{\left\lceil N_{f}\right\rceil, q^{\prime}}\right\rangle=1 \\
& \times\left|\left\langle\Phi_{\left\lfloor N_{f}\right\rfloor, m}\left|\hat{f}_{a}\right| \Phi_{\left\lceil N_{f}\right\rceil, q^{\prime}}\right\rangle\right|^{2} \delta\left(\zeta_{\left\lfloor N_{f}\right\rfloor}-\zeta_{\left\lceil N_{f}\right\rceil}+\varepsilon\right) \\
& +\frac{1}{N_{\text {orb }}} \sum_{a} \sum_{\substack{m^{\prime} \\
\left\langle\Phi_{\left\lceil N_{f}\right\rceil, m^{\prime}}\left|\hat{n}_{a}\right| \Phi_{\left\lceil N_{f}\right\rceil, m^{\prime}}\right\rangle=0}} \sum_{q=1}^{N_{\text {states }}\left(\left\lceil N_{f}\right\rceil+1\right)} w_{\left\lceil N_{f}\right\rceil, m^{\prime}} \\
& \times\left|\left\langle\Phi_{\left\lceil N_{f}\right\rceil, m^{\prime}}\left|\hat{f}_{a}\right| \Phi_{\left\lceil N_{f}\right\rceil+1, q}\right\rangle\right|^{2} \delta\left(\zeta_{\left\lceil N_{f}\right\rceil}-\zeta_{\left\lceil N_{f}\right\rceil+1}+\varepsilon\right)
\end{aligned}
$$

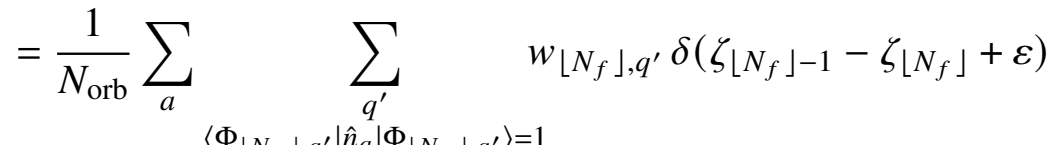

$$
\begin{aligned}
& \left\langle\Phi_{\left\lfloor N_{f}\right\rfloor, q^{\prime}}\left|\hat{n}_{a}\right| \Phi_{\left\lfloor N_{f}\right\rfloor, q^{\prime}}\right\rangle=1
\end{aligned}
$$

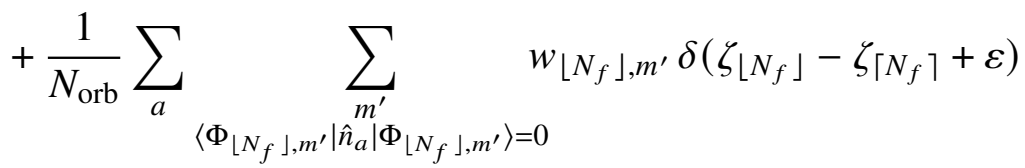

$$
\begin{aligned}
& +\frac{1}{N_{\text {orb }}} \sum_{a} \quad \sum_{q^{\prime}} \quad w_{\left\lceil N_{f}\right\rceil, q^{\prime}} \delta\left(\zeta_{\left\lfloor N_{f}\right\rfloor}-\zeta_{\left\lceil N_{f}\right\rceil}+\varepsilon\right) \\
& \left\langle\Phi_{\left\lceil N_{f}\right\rceil, q^{\prime}}\left|\hat{n}_{a}\right| \Phi_{\left\lceil N_{f}\right\rceil, q^{\prime}}\right\rangle=1 \\
& +\frac{1}{N_{\text {orb }}} \sum_{a} \sum_{\substack{m^{\prime} \\
\left\langle\Phi_{\left\lceil N_{f}\right\rceil, m^{\prime}}\left|\hat{n}_{a}\right| \Phi_{\left\lceil N_{f}\right\rceil, m^{\prime}}\right\rangle=0}} w_{\left\lceil N_{f}\right\rceil, m^{\prime}} \delta\left(\zeta_{\left\lceil N_{f}\right\rceil}-\zeta_{\left\lceil N_{f}\right\rceil+1}+\varepsilon\right)
\end{aligned}
$$




$$
\begin{aligned}
= & \frac{1}{N_{\text {orb }}}\left[\left\lfloor N_{f}\right\rfloor \sum_{i}^{N_{\text {states }}\left(\left\lfloor N_{f}\right\rfloor\right)} w_{\left\lfloor N_{f}\right\rfloor, i}\right] \delta\left(\zeta_{\left\lfloor N_{f}\right\rfloor-1}-\zeta_{\left\lfloor N_{f}\right\rfloor}+\varepsilon\right) \\
& +\frac{1}{N_{\text {orb }}}\left[\left(N_{\text {orb }}-\left\lfloor N_{f}\right\rfloor\right) \sum_{i}^{N_{\text {states }}\left\lfloor\left\lfloor N_{f}\right\rfloor\right)} w_{\left\lfloor N_{f}\right\rfloor, i}\right] \delta\left(\zeta_{\left\lfloor N_{f}\right\rfloor}-\zeta_{\left\lceil N_{f}\right\rceil}+\varepsilon\right) \\
& +\frac{1}{N_{\text {orb }}}\left[\left\lceil N_{f}\right\rceil \sum_{i}^{N_{\text {states }}\left(\left\lceil N_{f}\right\rceil\right)} w_{\left\lceil N_{f}\right\rceil, i}\right] \delta\left(\zeta_{\left\lfloor N_{f}\right\rfloor}-\zeta_{\left\lceil N_{f}\right\rceil}+\varepsilon\right) \\
& +\frac{1}{N_{\text {orb }}}\left[\left(N_{\text {orb }}-\left\lceil N_{f}\right\rceil\right) \sum_{i}^{N_{\text {states }}\left(\left\lceil N_{f}\right\rceil\right)} w_{\left\lceil N_{f}\right\rceil, i}\right] \delta\left(\zeta_{\left\lceil N_{f}\right\rceil}-\zeta_{\left\lceil N_{f}\right\rceil+1}+\varepsilon\right)
\end{aligned}
$$

At this point we insert eqs. (5.23) and (5.24), so that the spectral function simplifies to

$$
\begin{aligned}
A\left(\varepsilon, N_{f}\right)= & \frac{\left\lfloor N_{f}\right\rfloor}{N_{\text {orb }}}\left(1-n_{f}^{\mathrm{frac}}\right) \delta\left(\zeta\left\lfloor N_{f}\right\rfloor-1-\zeta_{\left\lfloor N_{f}\right\rfloor}+\varepsilon\right) \\
& +\frac{N_{\text {orb }}-\left\lfloor N_{f}\right\rfloor}{N_{\text {orb }}}\left(1-n_{f}^{\mathrm{frac}}\right) \delta\left(\zeta_{\left\lfloor N_{f}\right\rfloor}-\zeta_{\left\lceil N_{f}\right\rceil}+\varepsilon\right) \\
& +\frac{\left\lceil N_{f}\right\rceil}{N_{\text {orb }}} n_{f}^{\mathrm{frac}} \delta\left(\zeta\left\lfloor N_{f}\right\rfloor-\zeta_{\left\lceil N_{f}\right\rceil}+\varepsilon\right) \\
& +\frac{N_{\text {orb }}-\left\lceil N_{f}\right\rceil}{N_{\text {orb }}} n_{f}^{\mathrm{frac}} \delta\left(\zeta_{\left\lceil N_{f}\right\rceil}-\zeta_{\left\lceil N_{f}\right\rceil+1}+\varepsilon\right) .
\end{aligned}
$$

Hence, in the case of a non-integer occupancy $N_{f}$, the spectral function has a three-peak structure. The reason for this is that a non-integer occupancy $N_{f}$ can only be achieved through a mixed state of Slater determinants from two neighboring integer particle number sectors so that both particle number sectors contribute two $\delta$-peaks to the spectral function. Of the four contributions two peaks are at the same energy due to mutual excitations. Hence the spectral function for non-integer occupancy $N_{f}$ exhibits a total of three $\delta$-peaks. Their energies are

$$
\begin{aligned}
\Delta \zeta_{\text {low }} & =\zeta_{\left\lfloor N_{f}\right\rfloor}-\zeta_{\left\lfloor N_{f}\right\rfloor-1}=\varepsilon_{f}+U\left(\left\lfloor N_{f}\right\rfloor-1\right), \\
\Delta \zeta_{\text {center }} & =\zeta_{\left\lceil N_{f}\right\rceil}-\zeta_{\left\lfloor N_{f}\right\rfloor}=\varepsilon_{f}+U\left\lfloor N_{f}\right\rfloor \\
\Delta \zeta_{\text {high }} & =\zeta_{\left\lceil N_{f}\right\rceil+1}-\zeta_{\left\lceil N_{f}\right\rceil}=\varepsilon_{f}+U\left\lceil N_{f}\right\rceil .
\end{aligned}=E_{\mathrm{F}},
$$

Note that, according to eq. (5.32), the center peak is pinned to the Fermi level. Furthermore, we see that the peak separation is $U$.

The spectral function for a range of values for the energy parameter of the localized orbitals $\varepsilon_{f}$ is plotted in fig. 5.5. We plot the contributions from the particle removal excitation in purple and from the particle insertion excitation in orange. In the case of a fractional occupancy, we stack the contributions of both excitation types in the center peak. Below $\varepsilon_{f} / \Gamma=-21.0$, a constant occupancy 
5 The density of states of localized orbitals with fractional occupancy: study of a model system
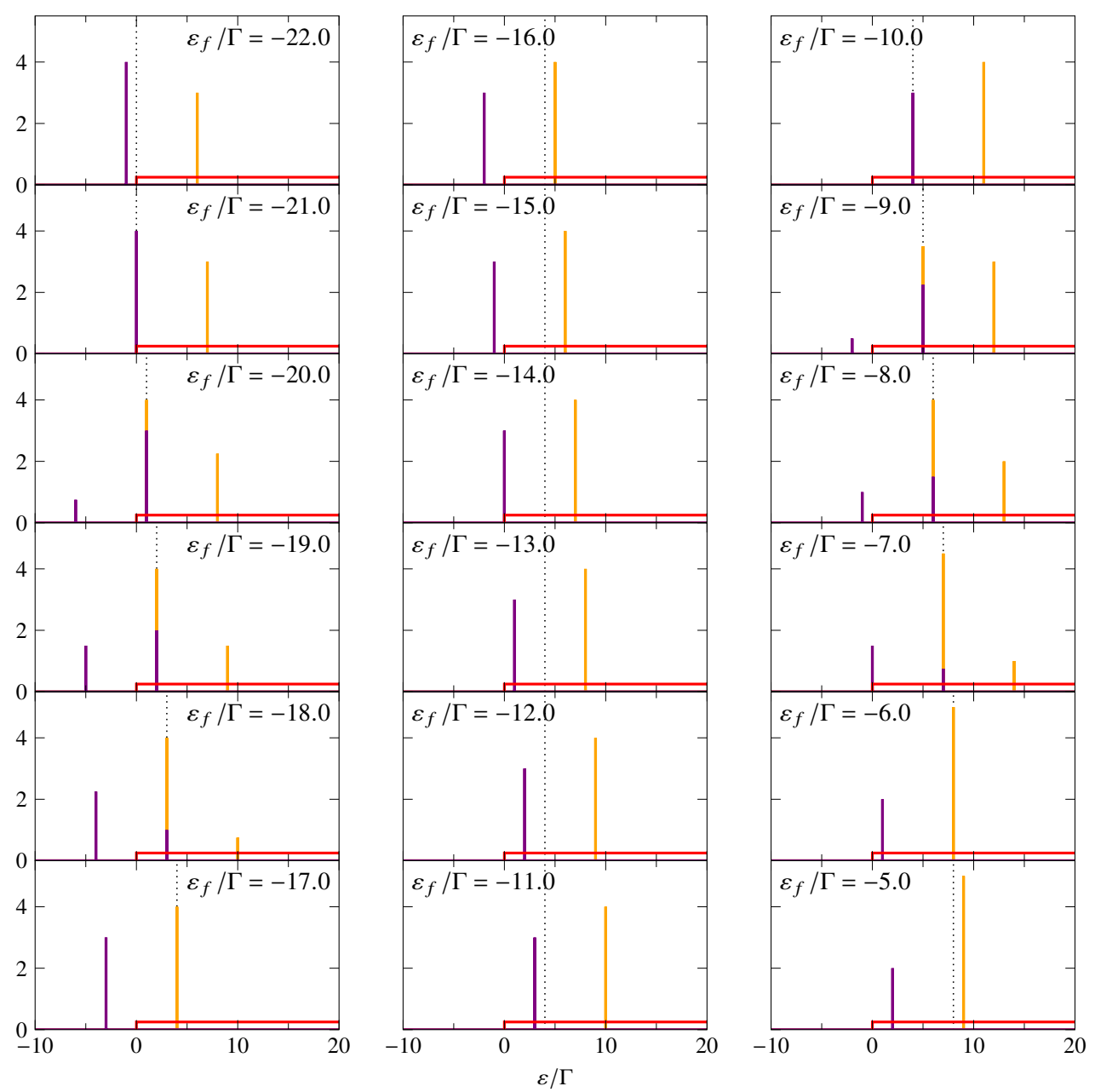

Figure 5.5: Spectral function $A_{\mathrm{loc}}\left(\varepsilon, N_{f}^{\mathrm{GS}}\right) \cdot N_{\text {orb }}$ of the impurity in the full many-body approach for various values of $\varepsilon_{f}$. Purple: contribution from the removal of a particle from the impurity. Orange: contribution from the addition of a particle to the impurity. In the center peak the contributions of particle insertion and particle removal excitations are stacked. Red: bath DOS. Model parameters: $U / \Gamma=7, D / \Gamma=1 / 4$. Total electron number: $N_{\text {tot }}=4$, number of localized orbitals: $N_{\text {orb }}=7$. The dotted black line marks the Fermi level. 
$N_{f}=4$ is found for the impurity while the bath states remain empty. When the lower $\delta$-peak of the spectral function hits the Fermi level at $\varepsilon_{f} / \Gamma=-21.0$, the first sector with a fractional occupancy of the impurity is entered, lowering the impurity's occupancy and increasing the bath's occupancy. In the fractional occupancy sector the spectral function clearly takes a three-peak structure, where with increasing $\varepsilon_{f}$ the upper peak shrinks and the lower peak grows. The center peak occupancy is gradually reduced to zero. While stepping through the sector of fractional occupancy, the impurity's total occupancy is reduced from 4 to 3 as an electron is shifted to the bath. The three peaks and the Fermi level move upward in energy while the center peak remains pinned to the Fermi level. At $\varepsilon_{f} / \Gamma=-17.0$ the sector of fractional occupancy is left and the sector of constant occupancy $\bar{N}_{f}=3$ is entered. There the spectral function again has a two-peak structure and the two peaks move to larger energies as $\varepsilon_{f}$ increases. At $\varepsilon_{f} / \Gamma=-10.0$ the next sector of fractional occupancy is reached.

\subsection{Hartree-Fock approach to the model system}

In this section we treat the model system in the Hartree-Fock approximation. Because we are only interested in ground state properties, we work in the limit of zero temperature, $T \rightarrow 0$. First, we determine the ground state total energy and the ground state ensemble of the impurity in the Hartree-Fock approximation. Second, from this ground state ensemble we compute the spectral function of the impurity. In the Hartree-Fock approach there are two possibilities to compute the energies of particle-insertion and particle-removal excitations of the states within the ground state ensemble. The excitation energies can be obtained from the Hamiltonian of the effective non-interacting system of the Hartree-Fock approximation or from the Hamiltonian of the original interacting system. Hence, we obtain two different spectral functions. We compare both spectral functions from the Hartree-Fock treatment with the spectral function of the impurity of the original interacting system.

In the Hartree-Fock approximation the Hamiltonian of an auxiliary noninteracting system is set up by taking the non-interacting part of the original impurity Hamiltonian and adding an effective potential term, see section 3.7.1. The effective potential represents the interaction between the localized orbitals on a mean-field level. In the case of our model system, the Hamiltonian for the impurity in the Hartree-Fock approximation reads

$$
\begin{aligned}
\hat{H}_{\mathrm{loc}}^{\mathrm{HF}} & =\sum_{a b} h_{a b} \hat{f}_{a}^{\dagger} \hat{f}_{b}+\sum_{a b c d}\left(U_{d a c b}-U_{d a b c}\right)\left\langle\hat{f}_{d}^{\dagger} \hat{f}_{c}\right\rangle_{\mathrm{HF}} \hat{f}_{a}^{\dagger} \hat{f}_{b} \\
& =\varepsilon_{f} \sum_{a} \hat{f}_{a}^{\dagger} \hat{f}_{a}+U \sum_{a b}\left(\left\langle\hat{f}_{a}^{\dagger} \hat{f}_{a}\right\rangle_{\mathrm{HF}} \hat{f}_{b}^{\dagger} \hat{f}_{b}-\left\langle\hat{f}_{a}^{\dagger} \hat{f}_{b}\right\rangle_{\mathrm{HF}} \hat{f}_{b}^{\dagger} \hat{f}_{a}\right),
\end{aligned}
$$

where we exploited the simplifications (5.8) and (5.9) of our model. The impurity is an open subsystem so that its occupancy can be fractional. A fractional occupancy is reflected in the elements $\left\langle\hat{f}_{a}^{\dagger} \hat{f}_{b}\right\rangle_{\mathrm{HF}}$ of the density matrix. This situation is analogous to the situation in DFT with the PBE0r hybrid 
5 The density of states of localized orbitals with fractional occupancy: study of a model system

functional, where the local orbitals of each atomic site comprise an open subsystem. The occupancy of the local orbitals can be fractional and the Hartree-Fock exchange contribution in the PBE0r hybrid functional is computed with the density matrix of the possibly fractionally occupied subsystem of local orbitals.

The Hartree-Fock approximation to the total energy of the original interacting system differs from the expectation value $\left\langle\hat{H}_{\mathrm{loc}}^{\mathrm{HF}}\right\rangle_{\mathrm{HF}}$ by the Hartree energy $E_{\mathrm{H}}$ and the exchange energy $E_{X}$, see eq. (3.88). In the remainder of the chapter we will use the following notation:

$$
E_{\mathrm{loc}}^{\mathrm{HF}}:=\left\langle\hat{H}_{\mathrm{loc}}\right\rangle_{\mathrm{HF}}=\left\langle\hat{H}_{\mathrm{loc}}^{\mathrm{HF}}\right\rangle_{\mathrm{HF}}-\left(E_{\mathrm{H}}+E_{\mathrm{X}}\right) .
$$

Let us express $E_{\mathrm{loc}}^{\mathrm{HF}}$ in terms of the occupancy numbers of the localized orbitals. The first term of eq. (5.54) is the total energy of the impurity in the effective non-interacting system. It is computed as the average of $\hat{H}_{\mathrm{loc}}^{\mathrm{HF}}$, eq. (5.53), over the ground state ensemble of the effective non-interacting system:

$$
\begin{aligned}
\left\langle\hat{H}_{\mathrm{loc}}^{\mathrm{HF}}\right\rangle_{\mathrm{HF}} & =\varepsilon_{f} \sum_{a}\left\langle\hat{n}_{a}\right\rangle_{\mathrm{HF}}+U \sum_{a b}\left(\left\langle\hat{n}_{a}\right\rangle_{\mathrm{HF}}\left\langle\hat{n}_{b}\right\rangle_{\mathrm{HF}}-\left\langle\hat{f}_{a}^{\dagger} \hat{f}_{b}\right\rangle_{\mathrm{HF}}\left\langle\hat{f}_{b}^{\dagger} \hat{f}_{a}\right\rangle_{\mathrm{HF}}\right) \\
& =\varepsilon_{f} \sum_{a}\left\langle\hat{n}_{a}\right\rangle_{\mathrm{HF}}+U \sum_{a b}\left(\left\langle\hat{n}_{a}\right\rangle_{\mathrm{HF}}\left\langle\hat{n}_{b}\right\rangle_{\mathrm{HF}}-\left\langle\hat{n}_{a}\right\rangle_{\mathrm{HF}}^{2} \delta_{a b}\right) \\
& =\varepsilon_{f} N_{f}+U N_{f}^{2}-U \sum_{a}\left\langle\hat{n}_{a}\right\rangle_{\mathrm{HF}}^{2}
\end{aligned}
$$

where

$$
N_{f}=\sum_{a}\left\langle\hat{n}_{a}\right\rangle_{\mathrm{HF}}
$$

To keep the notation simple we do not include a label "HF" for the total occupancy of the localized orbitals $N_{f}$. Moreover, the Hartree energy is

$$
E_{\mathrm{H}}=\frac{U}{2}\left(\sum_{a}\left\langle\hat{n}_{a}\right\rangle_{\mathrm{HF}}\right)^{2}=\frac{U}{2} N_{f}^{2}
$$

and the exchange energy reads

$$
E_{\mathrm{X}}=-\frac{U}{2} \sum_{a}\left\langle\hat{n}_{a}\right\rangle_{\mathrm{HF}}^{2} .
$$

After inserting eqs. (5.57), (5.59) and (5.60) into eq. (5.54) we obtain

$$
E_{\mathrm{loc}}^{\mathrm{HF}}=\varepsilon_{f} N_{f}+\frac{U}{2} N_{f}^{2}-\frac{U}{2} \sum_{a}\left\langle\hat{n}_{a}\right\rangle_{\mathrm{HF}}^{2} .
$$

Let us introduce a scaling factor $\eta$ for the exchange energy in analogy with the 
mixing factor for the Hartree-Fock exchange in the PBE0r hybrid functional:

$$
E_{\mathrm{loc}}^{\mathrm{HF}}=\varepsilon_{f} N_{f}+\frac{U}{2} N_{f}^{2}-\frac{\eta U}{2} \sum_{a}\left\langle\hat{n}_{a}\right\rangle_{\mathrm{HF}}^{2} .
$$

For $\eta=1$, we recover the exchange contribution of the original model. Note, that the exchange scaling factor $\eta$ is not equivalent to the exchange scaling within the PBE0r hybrid functional. Here, the value $\eta=1$ should rather be regarded as the value which corresponds to the choice of the exchange scaling in the PBE0r hybrid functional at which the correct ground state is produced in the DFT simulation. Because the exchange scaling factor in the PBE0r hybrid functional is guessed before the simulation, it can be chosen too small or too large. This deviation from the correct value of the exchange mixing in the PBE0r hybrid functional can be simulated by varying the value of $\eta$ in the model system. The case $\eta=0$ corresponds to the case of zero exchange mixing in the PBE0r hybrid functional.

The total energy of the combined system of bath and impurity reads

$$
\begin{aligned}
E_{\mathrm{tot}}^{\mathrm{HF}} & =E_{\mathrm{bath}}+E_{\mathrm{loc}}^{\mathrm{HF}} . \\
& =\frac{1}{2 D}\left(N_{\mathrm{tot}}-N_{f}\right)^{2}+\varepsilon_{f} N_{f}+\frac{U}{2} N_{f}^{2}-\frac{\eta U}{2} \sum_{a}\left\langle\hat{n}_{a}\right\rangle_{\mathrm{HF}}^{2},
\end{aligned}
$$

where we inserted the expression (5.6) for the bath energy.

In the upcoming sections we proceed as follows. First, we minimize the total energy $E_{\text {tot }}^{\mathrm{HF}}$ with respect to the occupancy $N_{f}$ of the impurity. In the process, we determine the ground state ensemble which minimizes $E_{\mathrm{tot}}^{\mathrm{HF}}$. Second, we compute the spectral function of the localized orbitals from the ground state ensemble. A detailed comparison of the results of the many-body treatment of the model and the results from the Hartree-Fock approach to the model is given in section 5.4.

\subsubsection{The ground state total energy}

To find the minimum of the ground state total energy of the model system in the Hartree-Fock approximation, we first minimize the energy $E_{\mathrm{loc}}^{\mathrm{HF}}$ over the set of occupancy numbers of the individual localized orbitals for a fixed $N_{f}$. In a second step, we minimize the total energy $E_{\text {tot }}^{\mathrm{HF}}$ with respect to $N_{f}$.

The first and second term of eq. (5.62) for $E_{\text {loc }}^{\mathrm{HF}}$ depend on the occupancy $N_{f}$, but do not care about the precise distribution of $N_{f}$ among the available localized orbitals. The third term is minimized if $\left\lfloor N_{f}\right\rfloor$ orbitals are filled and only one orbital has a fractional occupancy $n_{f}^{\text {frac }}=N_{f}-\left\lfloor N_{f}\right\rfloor$. Hence, the minimum total energy of the impurity for a given total occupancy $N_{f}$ is

$$
E_{\mathrm{loc}, \min }^{\mathrm{HF}}\left(N_{f}\right)=\varepsilon_{f} N_{f}+\frac{U}{2} N_{f}^{2}-\frac{\eta U}{2}\left\lfloor N_{f}\right\rfloor-\frac{\eta U}{2}\left(n_{f}^{\mathrm{frac}}\right)^{2} .
$$


5 The density of states of localized orbitals with fractional occupancy: study of a model system

In the case of an integer occupancy $\bar{N}_{f}$ the total energy is

$$
E_{\mathrm{loc}, \min }^{\mathrm{HF}}\left(\bar{N}_{f}\right)=\varepsilon_{f} \bar{N}_{f}+\frac{U}{2} \bar{N}_{f}^{2}-\frac{\eta U}{2} \bar{N}_{f}
$$

For $\eta=1$, the total energy (5.66) is the same as in the many-body calculation, see eq. (5.19) and eq. (5.14), respectively. Similarly, for a fractional occupancy and at $\eta=1$ the total energy (5.65) is identical to eq. (5.27) of the many-body approach.

We remark that we can use the energy difference of two neighboring integer occupancies,

$$
\begin{aligned}
\Delta E_{\mathrm{loc}, \min }^{\mathrm{HF}} & =E_{\mathrm{loc}, \min }^{\mathrm{HF}}\left(\bar{N}_{f}+1\right)-E_{\mathrm{loc}, \min }^{\mathrm{HF}}\left(\bar{N}_{f}\right) \\
& =\varepsilon_{f}+U \bar{N}_{f}+\frac{U}{2}(1-\eta),
\end{aligned}
$$

to rewrite the energy (5.65) as

$$
E_{\mathrm{loc}, \min }^{\mathrm{HF}}\left(N_{f}\right)=E_{\mathrm{loc}, \min }^{\mathrm{HF}}\left(\left\lfloor N_{f}\right\rfloor\right)+\Delta E_{\mathrm{loc}, \min }^{\mathrm{HF}} n_{f}^{\mathrm{frac}}-\frac{U}{2}(1-\eta)\left(1-n_{f}^{\mathrm{frac}}\right) n_{f}^{\mathrm{frac}} .
$$

Like in the expression for the energy of the localized orbitals in the many-body approach, $E_{\text {loc,min }}^{\mathrm{HF}}\left(N_{f}\right)$ has kinks at integer occupancy $\bar{N}_{f}$ for $\eta>0$, which become more prominent the larger the value of $\eta$.

The minimum of the total energy of the complete model for a given occupancy $N_{f}$ of the impurity reads

$$
E_{\mathrm{tot}, \text { min }}^{\mathrm{HF}}\left(N_{f}\right)=E_{\mathrm{bath}}\left(N_{f}\right)+E_{\mathrm{loc}, \min }^{\mathrm{HF}}\left(N_{f}\right) .
$$

Again, the ground state energy $E_{\mathrm{tot}}^{\mathrm{HF}, \mathrm{GS}}$ and the ground state occupancy $N_{f}^{\mathrm{GS}}$ are obtained by minimizing eq. (5.70) with respect to $N_{f}$,

$$
E_{\mathrm{tot}}^{\mathrm{HF}, \mathrm{GS}}=\min _{N_{f}} E_{\mathrm{tot}, \min }^{\mathrm{HF}}\left(N_{f}\right) .
$$

As an example, the dependence of the total energy $E_{\text {tot,min }}^{\mathrm{HF}}$ on the occupancy $N_{f}$ is depicted in fig. 5.6 for five different values of the exchange scaling factor $\eta$ for $\varepsilon_{f} / \Gamma=-19$. The parameters $U / \Gamma=7$ and $D \Gamma=1 / 4$ for the interaction strength and the density of states, respectively, are the same as for the manybody approach to the model system in section 5.2. For $\eta=0$ only the Hartree energy remains from the interaction part and the total energy takes a parabolic shape. When the exchange mixing is turned on, the values of the total energy decrease in general because the fraction $\eta$ of the self-interaction is subtracted. Furthermore, kinks form at integer occupancies.

In fig. 5.7, we show an example plot of the total energy versus the occupancy of the impurity for a range of different values for the parameter $\varepsilon_{f}$. In the example the exchange mixing is set to $\eta=1 / 4$. It is clear that the minima of the total energy shift towards larger $N_{f}$ for smaller values of $\varepsilon_{f}$, as a higher 


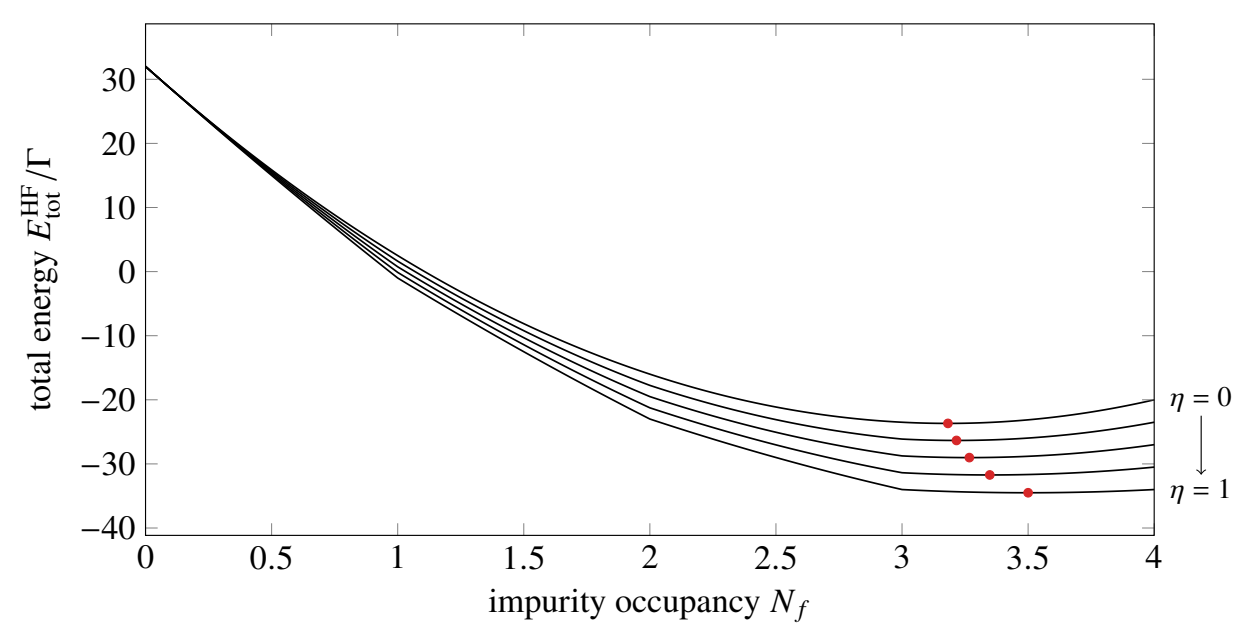

Figure 5.6: Minimum of the total energy $E_{\mathrm{tot}, \min }^{\mathrm{HF}}$ against the total occupancy of the localized orbitals $N_{f}$ for various values of the Hartree-Fock exchange mixing factor $\eta$. The minima of the curves are indicated by the red dots. Model parameters: $U / \Gamma=7, \varepsilon_{f} / \Gamma=-19, D \Gamma=1 / 4$.

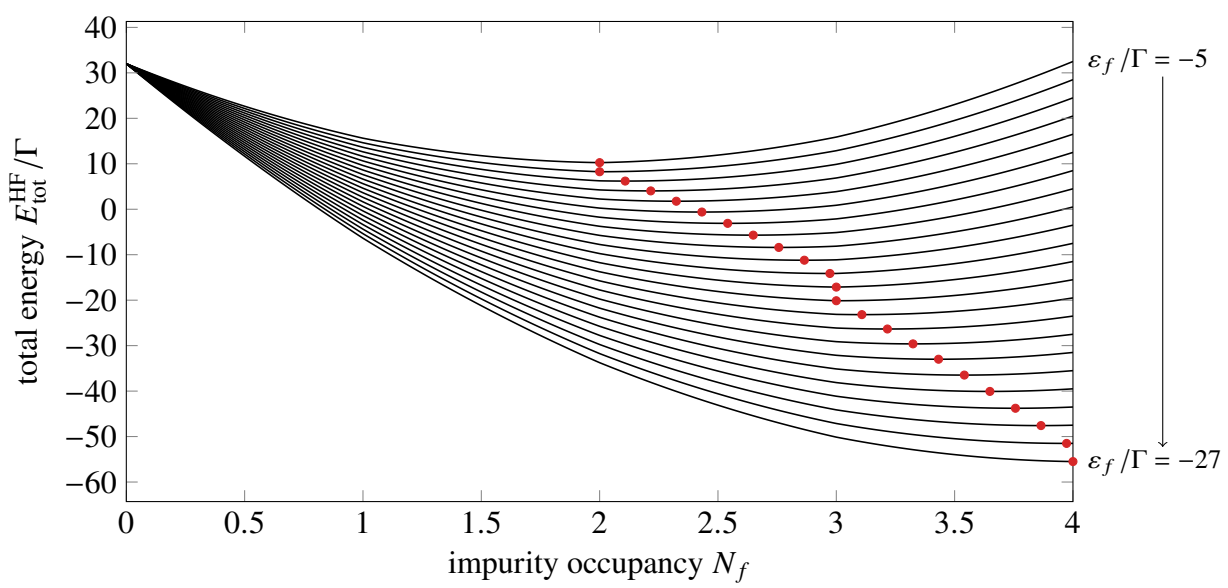

Figure 5.7: Dependence of the total energy $E_{\text {tot }}^{\mathrm{HF}}$ on the total occupancy of the localized orbitals $N_{f}$ for various values of $\varepsilon_{f}$. The minima of the curves are indicated by the red dots. Model parameters: $U / \Gamma=7$, $\eta=1 / 4, D \Gamma=1 / 4$. 
5 The density of states of localized orbitals with fractional occupancy: study of a model system

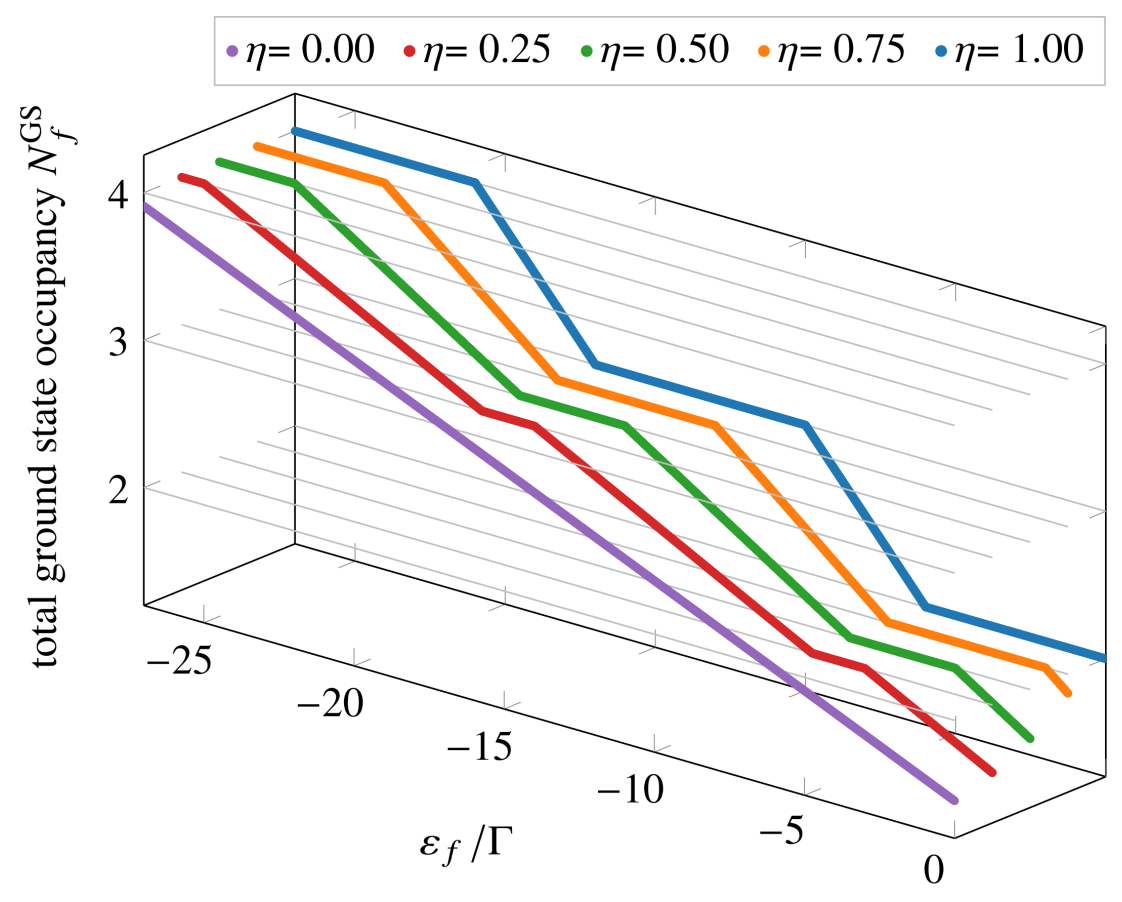

Figure 5.8: Dependence of the total ground state occupancy of the localized orbitals $N_{f}^{\mathrm{GS}}$ on $\varepsilon_{f}$ for various values of the Hartree-Fock exchange mixing factor $\eta$. Model parameters: $U / \Gamma=7, D \Gamma=1 / 4$.

occupancy of the impurity becomes energetically more favorable. Note that derivative discontinuities occur at integer values of $N_{f}$ and the curve of the energy minima is shaped such that an integer occupancy of the impurity occurs in the ground state for small ranges of $\varepsilon_{f}$. This can be seen at integer values of $N_{f}$, where the minima of $N_{f}$ are arranged vertically. By tuning the exchange mixing factor $\eta$ to larger values, integer ground state occupancies are realized for larger ranges of $\varepsilon_{f}$. This is evident from fig. 5.8, where the total ground state occupancy of the impurity $N_{f}^{\mathrm{GS}}$ is plotted against the energy parameter $\varepsilon_{f}$ for multiple values of the exchange scaling factor $\eta$. For $\eta=0, N_{f}^{\mathrm{GS}}$ takes a linear shape, where each value of $\varepsilon_{f}$ is assigned a value of $N_{f}^{\mathrm{GS}}$. At $\eta>0$ the introduction of kinks at integer $N_{f}^{\mathrm{GS}}$ leads to the formation of plateaus, where ranges of $\varepsilon_{f}$ lead to integer ground state occupancies. It can be seen that the plateau width increases with increasing $\eta$. Hence, for small exchange scaling $\eta$ fractional occupancies dominate, while for large $\eta$ integer occupancies minimize the total energy over broad ranges of $\varepsilon_{f}$. Note that the curve $N_{f}^{\mathrm{GS}}\left(\varepsilon_{f}\right)$ for $\eta=1$ is identical to the curve from the many-body approach in fig. 5.4.

\subsubsection{The spectral function in the Hartree-Fock approximation}

In the previous section we found that the total energy of the model system in the Hartree-Fock approximation is minimized, if, for an occupancy $N_{f}$ of the impurity, $\left\lfloor N_{f}\right\rfloor$ localized orbitals are fully occupied and at most one localized orbital has a fractional occupancy. From this information and with the ground 
state occupancy $N_{f}^{\mathrm{GS}}$ of the impurity we can infer the ensemble for the impurity which minimizes $E_{\text {tot,min }}^{\mathrm{HF}}\left(N_{f}\right)$. We have to choose which of the localized orbitals receive a full occupancy, which orbital is fractionally occupied, and which orbitals remain empty. This choice breaks the symmetry of the impurity system as the localized orbitals can no longer be treated on an equal footing. I. e. the dependence of the effective non-interacting Hamiltonian on the occupancy numbers of the localized orbitals leads to a broken-symmetry ensemble. An even distribution of the occupancy across all localized orbitals would instead lead to an unstable equilibrium.

In the following we state the ensemble of the impurity which minimizes $E_{\mathrm{tot}}^{\mathrm{HF}}$. Note that the same ensemble minimizes $\left\langle\hat{H}_{\mathrm{HF}}\right\rangle_{\mathrm{HF}}$. This means that the ground state ensemble of the effective non-interacting system of the HartreeFock approach gives rise to the Hartree-Fock approximation $E_{\text {tot }}^{\mathrm{HF}, \mathrm{GS}}$ of the ground state total energy of the interacting system. For simplicity we write the Slater determinants in the occupancy number basis in which the localized orbitals are ordered according to their occupancy. In the case of an integer ground state occupancy $\bar{N}_{f}^{\mathrm{GS}}$ the total energy $E_{\text {tot }}^{\mathrm{HF}}$ is minimized by a pure-state Slater determinant

$$
\left|\Phi_{\bar{N}_{f}^{\mathrm{GS}}}\right\rangle=|\underbrace{1, \ldots, 1}_{\bar{N}_{f}^{\mathrm{GS}}}, \underbrace{0, \ldots, 0}_{N_{\mathrm{orb}}-\bar{N}_{f}^{\mathrm{GS}}}\rangle .
$$

For a fractional occupancy $N_{f}^{\mathrm{GS}}$ we have an ensemble of a $\left\lfloor N_{f}^{\mathrm{GS}}\right\rfloor$-particle Slater determinant

$$
\left|\Phi_{\left\lfloor N_{f}^{\mathrm{GS}}\right\rfloor}\right\rangle=|\underbrace{1, \ldots, 1}_{\left\lfloor N_{f}^{\mathrm{GS}}\right\rfloor}, \underbrace{0, \ldots, 0}_{N_{\mathrm{orb}}-\left\lfloor N_{f}^{\mathrm{GS}}\right\rfloor}\rangle
$$

with weight $1-n_{f}^{\text {frac }}$ and a $\left\lceil N_{f}^{\mathrm{GS}}\right\rceil$-particle Slater determinant

$$
\left|\Phi_{\left\lceil N_{f}^{\mathrm{GS}}\right\rceil}\right\rangle=|\underbrace{1, \ldots, 1}_{\left\lceil N_{f}^{\mathrm{GS}}\right\rceil}, \underbrace{0, \ldots, 0}_{N_{\mathrm{orb}}-\left\lceil N_{f}^{\mathrm{GS}}\right\rceil}\rangle
$$

with weight $n_{f}^{\text {frac }}$.

We can now determine the spectral function of the localized orbitals. The excitation energies of a Slater determinant of the impurity ensemble due to the insertion or removal of a particle in an orbital $a$ are the differences of the energy eigenvalues associated with the Slater determinant and the excited Slater determinant that differs in the occupancy of the selected orbital $a$. Note that there are two possibilities to obtain the energy eigenvalues that are associated with each Slater determinant within the ensemble and the respective excitedstate Slater determinants, since we have two impurity Hamiltonians, $\hat{H}_{\mathrm{loc}}^{\mathrm{HF}}$ and $\hat{H}_{\text {loc }}$, respectively. This means that we can compute a spectral function for both of the Hamiltonians $\hat{H}_{\mathrm{loc}}^{\mathrm{HF}}$ and $\hat{H}_{\mathrm{loc}}$. The spectral function with respect to $\hat{H}_{\mathrm{loc}}^{\mathrm{HF}}$ is the spectral function of the localized orbitals of the effective non-interacting system. In contrast, the spectral function with respect to $\hat{H}_{\text {loc }}$ is the spectral function for the localized orbitals of the interacting system, but for the impurity 
5 The density of states of localized orbitals with fractional occupancy: study of a model system

ensemble that minimizes the total energy of the effective non-interacting model system.

\section{The spectral function with respect to $\hat{H}_{\text {loc }}^{\mathrm{HF}}$}

First, we determine the spectral function of the localized orbitals with respect to the effective non-interacting Hamiltonian $\hat{H}_{\mathrm{loc}}^{\mathrm{HF}}$. The Hamiltonian $\hat{H}_{\mathrm{loc}}^{\mathrm{HF}}$ can be written in diagonal form,

$$
\hat{H}_{\mathrm{loc}}^{\mathrm{HF}}=\sum_{a} \xi_{a} \hat{n}_{a}
$$

where the coefficients $\xi_{a}$ read

$$
\xi_{a}=\frac{\partial E_{\mathrm{loc}}^{\mathrm{HF}}}{\partial\left\langle\hat{n}_{a}\right\rangle_{\mathrm{HF}}}=\varepsilon_{f}+U N_{f}-\eta U\left\langle\hat{n}_{a}\right\rangle_{\mathrm{HF}} .
$$

Because the average orbital occupancies in the ensemble are either $1, n_{f}^{\mathrm{frac}}$, or 0 , we obtain three different values for the coefficients $\xi_{a}$. For $\left\langle\hat{n}_{a}\right\rangle_{\mathrm{HF}}=1$ we find the eigenvalue

$$
\xi_{\mathrm{occ}}=\varepsilon_{f}+U N_{f}-\eta U
$$

whereas for $\left\langle\hat{n}_{a}\right\rangle_{\mathrm{HF}}=0$, we obtain

$$
\xi_{\text {unocc }}=\varepsilon_{f}+U N_{f} .
$$

The fractional occupancy $\left\langle\hat{n}_{a}\right\rangle_{\mathrm{HF}}=n_{f}^{\mathrm{frac}}$ gives the eigenvalue

$$
\xi_{\text {frac }}=\varepsilon_{f}+U N_{f}-\eta U n_{f}^{\mathrm{frac}} .
$$

Moreover, for the parameter regimes of a fractional occupancy the Fermi level can be obtained from the minimization of the total energy expression (5.64) with respect to the fractional occupancy $n_{f}^{\text {frac }}$,

$$
\frac{\partial E_{\mathrm{tot}}^{\mathrm{HF}}}{\partial n_{f}^{\mathrm{frac}}}=-\frac{1}{D}\left(N_{\mathrm{tot}}-N_{f}\right)+\varepsilon_{f}+U N_{f}-\eta U n_{f}^{\mathrm{frac}} .
$$

After inserting eq. (5.5) into eq. (5.80) and exploiting the minimization condition $\partial E_{\mathrm{tot}}^{\mathrm{HF}} / \partial n_{f}^{\mathrm{frac}}=0$, we find the Fermi energy to be

$$
E_{\mathrm{F}}=\varepsilon_{f}+U N_{f}-\eta U n_{f}^{\mathrm{frac}}
$$

which is the same expression as eq. (5.79). Hence, we have found the important result that the fractionally occupied orbital is pinned to the Fermi level,

$$
\xi_{\text {frac }}=E_{\mathrm{F}} .
$$

Note that the Fermi level is identical to the Fermi level of the many-body approach, eq. (5.33), if $\eta=1$. 
With the energy coefficients (5.77) - (5.79) of the effective non-interacting Hamiltonian $\hat{H}_{\mathrm{loc}}^{\mathrm{HF}}$, we obtain the impurity spectral function $A_{\mathrm{loc}}^{\mathrm{HF}}(\varepsilon)$. For an integer occupancy we find

$$
A_{\mathrm{loc}}^{\mathrm{HF}}\left(\varepsilon, \overline{N_{f}}\right)=\frac{\overline{N_{f}}}{N_{\text {orb }}} \delta\left(\varepsilon-\xi_{\text {occ }}\right)+\frac{N_{\text {orb }}-\overline{N_{f}}}{N_{\text {orb }}} \delta\left(\varepsilon-\xi_{\text {unocc }}\right),
$$

and for a fractional occupancy we obtain

$$
\begin{aligned}
A_{\mathrm{loc}}^{\mathrm{HF}}\left(\varepsilon, N_{f}\right)= & \frac{\left\lfloor N_{f}\right\rfloor}{N_{\text {orb }}} \delta\left(\varepsilon-\xi_{\text {occ }}\right)+\frac{1-n_{f}^{\text {frac }}}{N_{\text {orb }}} \delta\left(\varepsilon-\xi_{\text {frac }}\right) \\
& +\frac{n_{f}^{\text {frac }}}{N_{\text {orb }}} \delta\left(\varepsilon-\xi_{\text {frac }}\right)+\frac{N_{\text {orb }}-\left\lceil N_{f}\right\rceil}{N_{\text {orb }}} \delta\left(\varepsilon-\xi_{\text {unocc }}\right) .
\end{aligned}
$$

In the case of a fractional occupancy we split the contribution of the fractionally occupied state into an unoccupied contribution and an occupied contribution. The former contribution is obtained from the particle insertion into the Slater determinant with $\left\lfloor N_{f}\right\rfloor$ particles and the latter contribution from the particle removal from the Slater determinant with $\left\lceil N_{f}\right\rceil$ particles.

We plot the impurity spectral function for a series of values for the model parameter $\varepsilon_{f}$ in fig. 5.9. Note that an exchange scaling of $\eta=1$ is chosen, which facilitates a comparison to the one-particle spectral function from the many-body approach. We start at $\varepsilon_{f} / \Gamma=-22$ with a ground-state occupancy of $N_{f}^{\mathrm{GS}}=4$. In this case the DOS has a two-peak shape, with one peak of occupied localized states and one peak of unoccupied localized states. The distance between the two peaks is $U$. When the energy parameter of the localized orbitals $\varepsilon_{f}$ is increased, both peaks move upward in energy until the occupied states hit the Fermi level at $\varepsilon_{f} / \Gamma=-21$. Further raising $\varepsilon_{f}$ then results in a splitting of the occupied orbitals: The fully occupied states are reduced by one and move downward in energy. On the other hand, the fractionally occupied localized state, which is pinned to the Fermi level, moves upward while its occupancy is transferred to the bath. When the fractionally occupied orbital is empty at $\varepsilon_{f} / \Gamma=-17$, it merges with the other empty orbitals and a two-peak DOS is reestablished. With increasing $\varepsilon_{f}$ the localized states again move upward in energy until the occupied orbitals hit the Fermi energy again at $\varepsilon_{f} / \Gamma=-10$ and the cycle of fractional occupancy of the impurity is repeated. 
5 The density of states of localized orbitals with fractional occupancy: study of a model system
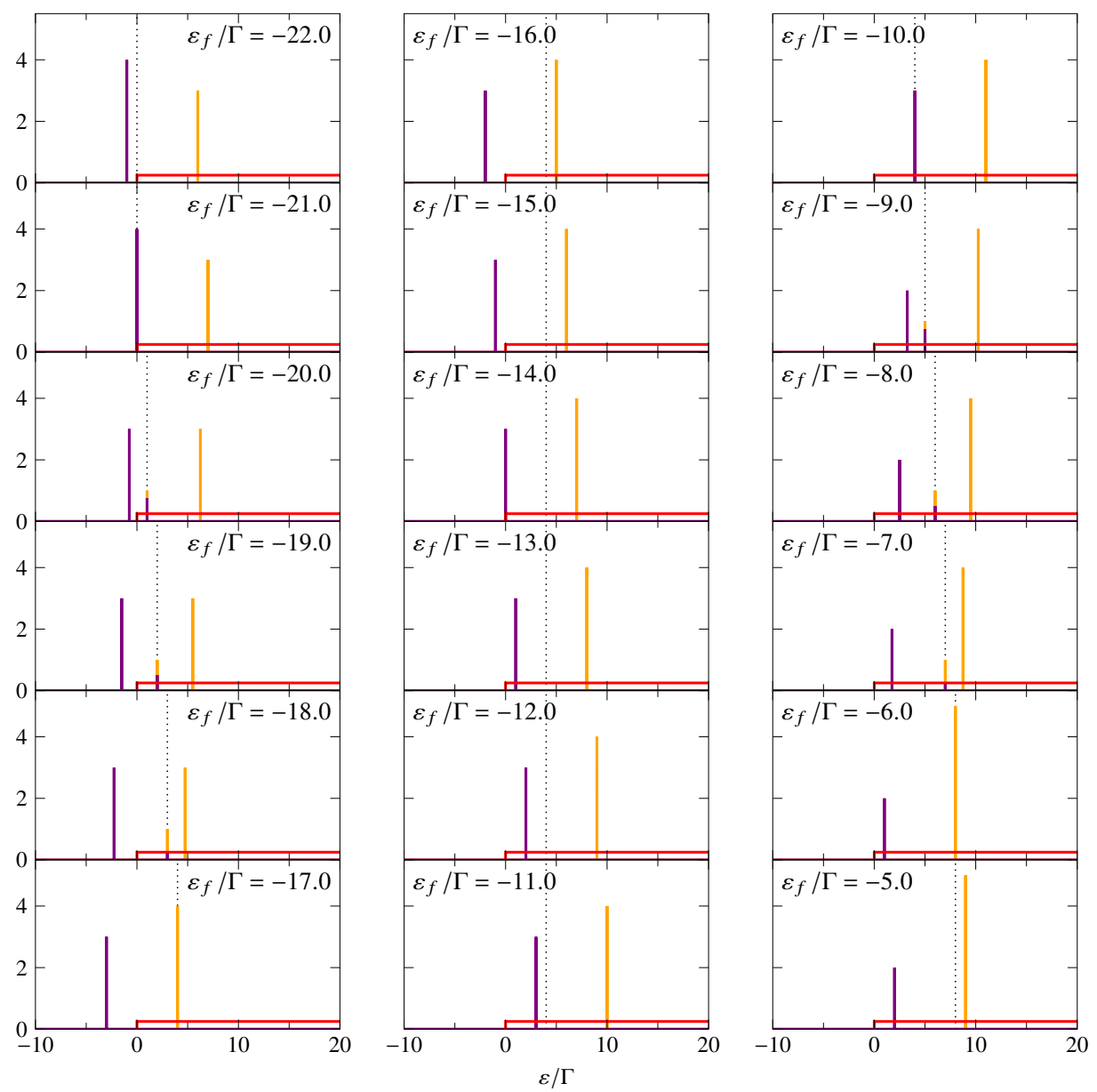

Figure 5.9: Spectral function $A_{\mathrm{loc}}^{\mathrm{HF}}\left(\varepsilon, N_{f}^{\mathrm{GS}}\right) \cdot N_{\mathrm{orb}}$ of the effective non-interacting impurity in the Hartree-Fock approach for various values of $\varepsilon_{f}$. Purple: contribution from the removal of an electron from the impurity, Orange: contribution from the addition of an electron to the impurity. In the peak of the fractionally occupied orbital (center peak) the contributions of particle insertion and particle removal excitations are stacked. Red: bath DOS. The Fermi level is indicated by the dotted black line. Model parameters: $U / \Gamma=7$, $\eta=1, D \Gamma=1 / 4, N_{\text {orb }}=7$. 
The spectral function with respect to $\hat{H}_{\text {loc }}$

Now we turn to the spectral function that is obtained from the ground state ensemble of the impurity in the Hartree-Fock approximation, but with excitation energies computed with the interacting impurity Hamiltonian $\hat{H}_{\text {loc }}$. The excitation energies are differences of the eigenenergies eq. (5.14) from neighboring particle number sectors. We use the symbol $B_{\text {loc }}^{\mathrm{HF}}$ to distinguish this spectral function from the spectral function in the preceding section. In the following we briefly list the spectral functions of the individual localized orbitals.

For an integer ground state occupancy $\bar{N}_{f}$ of the impurity, the ground state is a single Slater determinant. For each localized occupied orbital we find the same spectral function

$$
B_{\mathrm{loc}, \mathrm{occ}}^{\mathrm{HF}}\left(\varepsilon, \bar{N}_{f}\right)=\delta\left(\varepsilon+\zeta_{\bar{N}_{f}-1}-\zeta_{\bar{N}_{f}}\right) .
$$

Similarly, we obtain the spectral function

$$
B_{\text {loc,unocc }}^{\mathrm{HF}}\left(\varepsilon, \bar{N}_{f}\right)=\delta\left(\varepsilon+\zeta_{\bar{N}_{f}}-\zeta_{\bar{N}_{f}+1}\right)
$$

for each of the unoccupied localized orbitals. In the case of a fractional ground state occupancy $N_{f}^{\mathrm{GS}}$ of the impurity, we discriminate the spectral functions of occupied, unoccupied, and the fractionally occupied orbital. The ground state ensemble of the impurity comprises two Slater determinants with their associated weights. Each Slater determinant gives one contribution to the spectral function of an individual orbital. The spectral functions read

$$
\begin{aligned}
& B_{\text {loc,occ }}^{\mathrm{HF}}\left(\varepsilon, N_{f}\right)=(1\left.-n_{f}^{\mathrm{frac}}\right) \delta\left(\varepsilon+\zeta_{\left\lfloor N_{f}\right\rfloor-1}-\zeta_{\left\lfloor N_{f}\right\rfloor}\right) \\
&+n_{f}^{\mathrm{frac}} \delta\left(\varepsilon+\zeta_{\left\lfloor N_{f}\right\rfloor}-\zeta_{\left\lceil N_{f}\right\rceil}\right), \\
& B_{\text {loc,unocc }}^{\mathrm{HF}}\left(\varepsilon, N_{f}\right)=\left(1-n_{f}^{\mathrm{frac}}\right) \delta\left(\varepsilon+\zeta_{\left\lfloor N_{f}\right\rfloor}-\zeta_{\left\lceil N_{f}\right\rceil}\right) \\
&+n_{f}^{\mathrm{frac}} \delta\left(\varepsilon+\zeta_{\left\lceil N_{f}\right\rceil}-\zeta_{\left\lceil N_{f}\right\rceil+1}\right),
\end{aligned}
$$

and

$$
\begin{aligned}
B_{\mathrm{loc}, \mathrm{Hrac}}^{\mathrm{HF}}\left(\varepsilon, N_{f}\right)=(1 & \left.-n_{f}^{\mathrm{frac}}\right) \delta\left(\varepsilon+\zeta_{\left\lfloor N_{f}\right\rfloor}-\zeta_{\left\lceil N_{f}\right\rceil}\right) \\
& +n_{f}^{\mathrm{frac}} \delta\left(\varepsilon+\zeta_{\left\lfloor N_{f}\right\rfloor}-\zeta_{\left\lceil N_{f}\right\rceil}\right)
\end{aligned}
$$

for a fully occupied, an unoccupied, and the fractionally occupied localized orbital, respectively. The first contribution in the spectral function for the fractionally occupied orbital comes from the particle insertion excitation and is the contribution in which the fractionally occupied orbital is empty. The second contribution comes from the particle removal excitation and is the contribution in which the fractionally occupied orbital is occupied. Again, the fractionally occupied orbital is found at the Fermi level, which is given by eq. (5.32).

The total spectral function of the impurity is obtained by averaging over the individual spectral functions of the localized orbitals. It is identical to the 
5 The density of states of localized orbitals with fractional occupancy: study of a model system

spectral function of the many-particle calculation, eq. (5.41) for an integer occupancy and eq. (5.48) for a fractional occupancy of the impurity, respectively.

\section{4 $\mid$ Discussion of the spinless model}

In the preceding sections we studied our spinless model system with two different approaches: a many-body treatment and in the Hartree-Fock approximation. In particular, we calculated the ground state total energy, the ground state ensemble of the impurity, and the ground state spectral function of the impurity. We compare the results from both approaches in the following.

For an integer occupancy $\bar{N}_{f}$ of the impurity, in the many-body treatment the total energy of the model system is minimized by any Slater determinant of the localized orbitals with particle number $\bar{N}_{f}$. To satisfy the maximum entropy principle, all $\bar{N}_{f}$-particle Slater determinants have the same weight in the impurity ensemble. The weights of Slater determinants with other particle numbers are zero. In constrast, in the Hartree-Fock approximation, the minimum total energy of the impurity for an integer occupancy $\bar{N}_{f}$ is obtained for a single pure-state Slater determinant with $\bar{N}_{f}$ particles. The symmetry with respect to the individual localized orbitals is broken. An ensemble for the impurity that is symmetric in the localized orbitals does not minimize the total energy. In the Hartree-Fock approximation the total energy of the impurity is the average expectation value of the interacting Hamiltonian $\hat{H}_{\text {loc }}$ over the impurity ensemble that minimizes the total energy, $E_{\text {loc }}^{\mathrm{HF}}=\left\langle\hat{H}_{\mathrm{loc}}\right\rangle_{\mathrm{HF}}$. At an exchange scaling $\eta=1$, the contribution of the impurity to the ground state total energy in the Hartree-Fock approximation, see eq. (5.66), is the same as in the many-particle approach, see eq. (5.19) together with eq. (5.14). This is because Slater determinants are eigenstates of $\hat{H}_{\text {loc }}$ and all $\bar{N}_{f}$-particle Slater determinants have the same energy with respect to $\hat{H}_{\text {loc }}$.

For a fractional impurity occupancy $N_{f}$, in the many-body approach the total energy of the impurity is minimized by an ensemble of Slater determinants, in which only the Slater determinants with $\left\lfloor N_{f}\right\rfloor$ or $\left\lceil N_{f}\right\rceil$ particles can have non-zero weights. Moreover, the ratio of the weights of the $\left\lfloor N_{f}\right\rfloor$-particle Slater determinants to the weights of the $\left\lceil N_{f}\right\rceil$-particle Slater determinants is $\left(1-n_{f}^{\text {frac }}\right) / n_{f}^{\text {frac }}$, where $n_{f}^{\text {frac }}=N_{f}-\left\lfloor N_{f}\right\rfloor$. The impurity ensemble that satisfies the maximum entropy principle is symmetric with respect to all localized orbitals, i. e. all $\left\lfloor N_{f}\right\rfloor$-particle Slater determinants have the same weight and all $\left\lceil N_{f}\right\rceil$-particle Slater determinants have the same weight. In the Hartree-Fock approximation, again, the symmetry with respect to the localized orbitals is broken, such that the total energy is minimized by an impurity ensemble in which only one $\left\lfloor N_{f}\right\rfloor$-particle Slater determinant and one $\left\lceil N_{f}\right\rceil$-particle Slater determinant have non-zero weights. In the $\left\lceil N_{f}\right\rceil$-particle Slater determinant the same orbitals have to be occupied that also are occupied in the $\left\lfloor N_{f}\right\rfloor$-particle Slater determinant. The weight ratio of the $\left\lfloor N_{f}\right\rfloor$-particle Slater determinant and the $\left\lceil N_{f}\right\rceil$-particle Slater determinant is $\left(1-n_{f}^{\text {frac }}\right) / n_{f}^{\text {frac }}$. Because Slater determinants are eigenstates to the interacting Hamiltonian $\hat{H}_{\text {loc }}$, the impurity ensemble from the Hartree-Fock approximation at an exchange scaling $\eta=1$ 
gives rise to the same total energy of the impurity with respect to the interacting Hamiltonian $\hat{H}_{\mathrm{loc}}, E_{\mathrm{loc}}^{\mathrm{HF}}=\left\langle\hat{H}_{\mathrm{loc}}\right\rangle_{\mathrm{HF}}$, as the ensemble from the many-body approach. This is evident from a comparison of eq. (5.65) with eq. (5.27).

We proceed with a comparison of the spectral functions of the localized orbitals as obtained from the many-body treatment and from the Hartree-Fock approximation. In both approaches the spectral functions are obtained from the impurity ensembles that minimize the total energy of the model system and yield a ground state impurity occupancy $N_{f}^{\mathrm{GS}}$. For conciseness we restrict our discussion to the average spectral functions over all localized orbitals, which contain all relevant excitation energies.

In the Hartree-Fock approach we can obtain spectral functions for the localized orbitals in two different ways, since the excitation energies for particle insertion and particle removal can be computed with $\hat{H}_{\text {loc }}^{\mathrm{HF}}$ and also with $\hat{H}_{\text {loc }}$. First, we discuss the spectral function with respect to $\hat{H}_{\mathrm{loc}}^{\mathrm{HF}}$, which is the spectral function of the localized orbitals in the effective non-interacting system of the Hartree-Fock approach. The Hamiltonian $\hat{H}_{\mathrm{loc}}^{\mathrm{HF}}$ is non-interacting and the excitation energies are given by the set of one-particle energy levels $\{\xi\}$, see eqs. (5.75) and (5.76). Importantly, an energy level $\xi_{a}$ of a one-particle state $a$ depends on the average occupancy $\left\langle\hat{n}_{a}\right\rangle_{\mathrm{HF}}$ of the orbital $a$ within the impurity ensemble.

For an integer occupancy $\bar{N}_{f}$, the impurity state is a pure-state Slater determinant and the mean-field occupancies of the localized orbitals $\left\langle\hat{n}_{a}\right\rangle_{\mathrm{HF}}$ can be either 0 or 1 . In this case the energies $\{\xi\}$ can take two different values, depending on the occupancy of the respective orbital. These energies are equal to the energies of particle-insertion excitations and particle-removal excitations of the impurity Slater determinant with respect to the interacting Hamiltonian $\hat{H}_{\text {loc }}$. As a consequence, we find the same average spectral function over all impurity orbitals as in the many-body approach.

For a fractional occupancy $N_{f}$ the energy levels in the set $\{\xi\}$ take three different values, since we have $\left\lfloor N_{f}\right\rfloor$ occupied orbitals, $\left\lceil N_{f}\right\rceil$ unoccupied orbitals, and one fractionally occupied localized orbital. The spectral function differs from the spectral function of the many-particle approach. In both spectral functions, a $\delta$-peak is found at the Fermi level, which originates from mutual excitations between the $\left\lfloor N_{f}\right\rfloor$-particle Slater determinants and the $\left\lceil N_{f}\right\rceil$-particle Slater determinants of the impurity ensemble. Moreover, in the spectral function of the Hartree-Fock approximation this peak describes the energy level that belongs to the fractionally occupied localized orbital. This state can host one electron. A major difference between the two spectral functions is found in the energy distances of the excitation peaks from the Fermi level. In the spectral function of the Hartree-Fock approximation with respect to $\hat{H}_{\text {loc }}^{\mathrm{HF}}$, the fully occupied energy levels are found at $E_{\mathrm{F}}-\eta U\left(1-n_{f}^{\text {frac }}\right)$ and the unoccupied levels are found at $E_{\mathrm{F}}+\eta U n_{f}^{\text {frac }}$. This is in stark contrast to the lower and upper excitation peaks in the spectral function of the many-particle approach, which are found at energies $E_{\mathrm{F}}-U$ and $E_{\mathrm{F}}+U$, respectively.

Let us emphasize the reason for the difference in the peak distances of the spectral function for the effective non-interacting system and the spectral func- 
5 The density of states of localized orbitals with fractional occupancy: study of a model system

tion of the many-body approach. In the spectral function for the effective non-interacting system, the fractional occupancy $n_{f}^{\text {frac }}$ enters in the one-particle energy $\xi_{\text {frac }}$ of the fractionally occupied localized orbital. The value of $n_{f}^{\text {frac }}$ linearly interpolates the position of the fractionally occupied energy level between the energy levels of the fully occupied and the completely empty orbitals. In contrast, in the spectral function of the many-body calculation, the excitation energies do not depend on the fractional occupancy $n_{f}^{\text {frac }}$. However, the value of $n_{f}^{\text {frac }}$ affects the prefactors of the $\delta$-peaks, and thus the probability to find a particle or hole, respectively, with a given energy in the system.

The other possibility to obtain a spectral function from the impurity ensemble of the Hartree-Fock approximation is to calculate the excitation energies of the Slater determinants within the impurity ensemble with the interacting Hamiltonian $\hat{H}_{\text {loc }}$. In this case, the excitation energies are identical to those in the many-body approach and the same average spectral function over all localized orbitals as in the many-body approach is obtained. Note, however, that the spectral functions of the individual localized orbitals differ from those of the many-body approach since the symmetry of the localized orbitals is broken in the Hartree-Fock approach.

Before we come to a detailed comparison of the spectral functions of our model system with the DOSs of the rare-earth hexaborides of our DFT simulations with the PBE0r hybrid functional, we extend the so far spinless model system to a spin-dependent model. This is done in the upcoming sections.

\subsection{Spin-resolved model system with Hund's cou- pling}

Our DFT simulations of the rare-earth hexaborides are spin-resolved and exhibit a broken spin symmetry for $\mathrm{CeB}_{6}, \mathrm{PrB}_{6}$, and $\mathrm{NdB}_{6}$, for which the $4 f$ shell is partially occupied. Hence, to allow for a rigorous comparison of the DFT DOSs and the spectral function of the model system, a spin-resolved version of the model is desirable. In the following we extend our original spinless model for the rare-earth site to a spin-resolved model with a Hund's coupling that favors the occupancy of one spin direction. Again, we calculate the spectral function in a many-particle treatment of the model and in the Hartree-Fock approximation.

For our spin-dependent model we take the same bath system as before, see section 5.1.1. The localized orbitals of the impurity shall be spin-dependent. We replace the orbital indices of the impurity Hamiltonian (5.7) by a combination of a quantum number $m$, which labels the atomic orbital, and a spin index $\sigma$ in the following way: $a \rightarrow m \sigma, b \rightarrow m^{\prime} \sigma^{\prime}, c \rightarrow m^{\prime \prime} \sigma^{\prime \prime}$, and $d \rightarrow m^{\prime \prime \prime} \sigma^{\prime \prime \prime}$. Moreover, we introduce the following simplifications to the impurity Hamiltonian:

1. Similarly to the spinless model, we set the matrix elements of the non- 
interacting part of the impurity Hamiltonian to

$$
h_{m \sigma m^{\prime} \sigma^{\prime}}=\varepsilon_{f} \delta_{m m^{\prime}} \delta_{\sigma \sigma^{\prime}} .
$$

2. We use a simplified interaction tensor with tensor elements

$$
\begin{aligned}
U_{m \sigma m^{\prime} \sigma^{\prime} m^{\prime \prime} \sigma^{\prime \prime} m^{\prime \prime \prime} \sigma^{\prime \prime \prime}=} & U \delta_{m m^{\prime \prime}} \delta_{m^{\prime} m^{\prime \prime \prime}} \delta_{\sigma \sigma^{\prime \prime}} \delta_{\sigma^{\prime} \sigma^{\prime \prime \prime}} \\
& +J \delta_{m m^{\prime \prime \prime}} \delta_{m^{\prime} m^{\prime \prime}}\left(1-\delta_{m m^{\prime}}\right) \delta_{\sigma \sigma^{\prime \prime}} \delta_{\sigma^{\prime} \sigma^{\prime \prime \prime}} \delta_{\sigma \sigma^{\prime}} .
\end{aligned}
$$

In this way we reduce the interaction to direct interaction integrals of the form $U_{m m^{\prime} m m^{\prime}}=U$ and exchange integrals of the form $U_{m m^{\prime} m^{\prime} m}=$ $J$, if $m \neq m^{\prime}$. The direct interaction integrals are typically larger than the exchange integrals, $U>J$. Note that the Kronecker symbols $\delta_{\sigma \sigma^{\prime \prime}}$ and $\delta_{\sigma^{\prime} \sigma^{\prime \prime \prime}}$ emerge as a property of the interaction tensor for atomic orbitals, as described in section 3.4. The last Kronecker symbol $\delta_{\sigma \sigma^{\prime}}$ is introduced to keep the interaction Hamiltonian in a simple density-density interaction form, as we will see shortly. It excludes so-called spin-flip terms, which, for example, also occur in the Kanamori Hamiltonian, see e.g. [149, $150]$.

With the above simplification for the interaction tensor, the interaction part of the impurity Hamiltonian becomes

$$
\begin{aligned}
\hat{H}_{\text {loc,int }} & =\frac{1}{2} \sum_{m m^{\prime} m^{\prime \prime} m^{\prime \prime \prime}} \sum_{\sigma \sigma^{\prime} \sigma^{\prime \prime} \sigma^{\prime \prime \prime}} U_{m \sigma m^{\prime} \sigma^{\prime} m^{\prime \prime} \sigma^{\prime \prime} m^{\prime \prime \prime} \sigma^{\prime \prime \prime}} \hat{f}_{m \sigma}^{\dagger} \hat{f}_{m^{\prime} \sigma^{\prime}}^{\dagger} \hat{f}_{m^{\prime \prime \prime} \sigma^{\prime \prime \prime}} \hat{f}_{m^{\prime \prime} \sigma^{\prime \prime}} \\
& =\frac{U}{2} \sum_{m m^{\prime} \sigma \sigma^{\prime}} \sum_{m \sigma} \hat{f}_{m \sigma}^{\dagger} \hat{f}_{m^{\prime} \sigma^{\prime}}^{\dagger} \hat{f}_{m^{\prime} \sigma^{\prime}} \hat{f}_{m \sigma}+\frac{J}{2} \sum_{\substack{m m^{\prime} \\
m \neq m^{\prime}}} \sum_{\sigma} \hat{f}_{m \sigma}^{\dagger} \hat{f}_{m^{\prime} \sigma}^{\dagger} \hat{f}_{m \sigma} \hat{f}_{m^{\prime} \sigma} \\
& =\frac{U}{2} \sum_{m m^{\prime} \sigma} \hat{n}_{m \sigma} \hat{n}_{m^{\prime} \bar{\sigma}}+\frac{U-J}{2} \sum_{\substack{m m^{\prime} \sigma \\
m \neq m^{\prime}}} \hat{n}_{m \sigma} \hat{n}_{m^{\prime} \sigma}
\end{aligned}
$$

where $\bar{\sigma}$ denotes the opposite spin of $\sigma$. As we can see, we retain densitydensity interaction terms only. The parameter $J$ represents Hund's coupling. It ensures that the total spin of the localized orbitals should be maximized in accordance with Hund's rules. The complete impurity Hamiltonian reads

$$
\begin{aligned}
\hat{H}_{\mathrm{loc}} & =\varepsilon_{f} \sum_{m \sigma} \hat{n}_{m \sigma}+\frac{U}{2} \sum_{m m^{\prime} \sigma} \hat{n}_{m \sigma} \hat{n}_{m^{\prime} \bar{\sigma}}+\frac{U-J}{2} \sum_{\substack{m m^{\prime} \sigma \\
m \neq m^{\prime}}} \hat{n}_{m \sigma} \hat{n}_{m^{\prime} \sigma} \\
& =\varepsilon_{f} \hat{N}_{f}+\frac{U}{2} \sum_{\sigma} \hat{N}_{\sigma} \hat{N}_{\bar{\sigma}}+\frac{U-J}{2} \sum_{\sigma} \hat{N}_{\sigma}^{2}-\frac{U-J}{2} \hat{N}_{f},
\end{aligned}
$$


5 The density of states of localized orbitals with fractional occupancy: study of a model system

where we introduced the total occupancy number operator for spin $\sigma \in\{\uparrow, \downarrow\}$,

$$
\hat{N}_{\sigma}=\sum_{m} \hat{n}_{m \sigma}
$$

The eigenvalue of the operator $\hat{N}_{\sigma}$ is the integer particle number $\bar{N}_{\sigma}$ for spin $\sigma$. Summing over both spin directions gives the total occupancy number of the localized orbitals, $\bar{N}_{f}=\bar{N}_{\uparrow}+\bar{N}_{\downarrow}$. The Hamiltonian (5.96) is diagonal in the occupancy number basis of the spin orbitals and has eigenvalues

$$
\begin{aligned}
\zeta_{\bar{N}_{\uparrow}, \bar{N}_{\downarrow}} & =\varepsilon_{f} \bar{N}_{f}+U \bar{N}_{\uparrow} \bar{N}_{\downarrow}+\frac{U-J}{2}\left(\bar{N}_{\uparrow}^{2}+\bar{N}_{\downarrow}^{2}\right)-\frac{U-J}{2} \bar{N}_{f} \\
& =\varepsilon_{f} \bar{N}_{f}+\frac{U-J}{2} \bar{N}_{f}^{2}+J \bar{N}_{\uparrow} \bar{N}_{\downarrow}-\frac{U-J}{2} \bar{N}_{f} \\
& =\varepsilon_{f} \bar{N}_{f}+\frac{U-J}{2} \bar{N}_{f}\left(\bar{N}_{f}-1\right)+J \bar{N}_{\uparrow} \bar{N}_{\downarrow} .
\end{aligned}
$$

Only the last term of eq. (5.100) depends on the distribution of the $\bar{N}_{f}$ electrons on the two spin directions. It is minimal if either $\bar{N}_{\uparrow}=\bar{N}_{f}, \bar{N}_{\downarrow}=0$, or $\bar{N}_{\uparrow}=0, \bar{N}_{\downarrow}=\bar{N}_{f}$.

\subsection{Many-body approach to the spin-resolved model}

In this section, we analyze the spin-resolved model in a many-particle approach. We proceed analogously to our analysis of the spinless model. First, we search for the minimum of the total energy of the impurity for a given total occupancy $N_{f}$. We then determine the ensemble of the impurity that minimizes the total energy of the impurity for the given total occupancy. Subsequently, for this ensemble we derive the impurity spectral function.

\subsubsection{The ground state total energy in the many-body approach}

The impurity Hamiltonian $\hat{H}_{\text {loc }}$, eq. (5.96), is diagonal in the occupancy number basis of the spin orbitals of the impurity. The Slater determinants that are constructed from these spin orbitals in the occupancy number basis are eigenstates to $\hat{H}_{\text {loc }}$. Because the impurity is an open system, the total energy of the impurity in general is minimized by an ensemble of these Slater determinants. In the following, we write $w_{M_{\uparrow}, i_{\uparrow} ; M_{\downarrow}, i_{\downarrow}}$ for the weight of a Slater determinant within the ensemble. The first and the third index $\left(M_{\uparrow}\right.$ and $\left.M_{\downarrow}\right)$ indicate the particle number in each of the two spin directions, and the two additional indices $i_{\uparrow}, i_{\downarrow}$ label all combinations to fill $M_{\uparrow}$ electrons in the orbitals in the spin-up direction and $M_{\downarrow}$ electrons in the spin-down direction, respectively. If for a spin direction $M_{\sigma}=0$, we drop the index $i_{\sigma}$ as there is only one possibility for having all orbitals with this spin unoccupied.

In the following we restrict our discussion to the case of less-than-half filling of the localized orbitals, which covers the situation in the light rare-earth 
hexaborides. Because of Hund's coupling, in the ground state only ensemble weights of those Slater determinants are non-zero in which only one spin direction is occupied. The minimum energy for an integer occupancy $\bar{N}_{f}$ takes the form

$$
E_{\mathrm{loc}, \min }\left(\{w\}, \bar{N}_{f}\right)=\sum_{i_{\uparrow}=1}^{N_{\text {states }}^{\sigma}\left(\bar{N}_{f}\right)} w_{\bar{N}_{f}, i_{\uparrow} ; 0} \zeta_{\bar{N}_{f}, 0}+\sum_{i_{\downarrow}=1}^{N_{\text {states }}^{\sigma}\left(\bar{N}_{f}\right)} w_{0 ; \bar{N}_{f}, i_{\downarrow}} \zeta_{0, \bar{N}_{f}},
$$

where $N_{\text {states }}^{\sigma}\left(\bar{N}_{f}\right)$ is the number of possibilities to fill $\bar{N}_{f}$ electrons of the same spin into the localized orbitals. Note that $\zeta_{\bar{N}_{f}, 0}=\zeta_{0, \bar{N}_{f}}$ and the weights sum to 1 . Hence,

$$
E_{\mathrm{loc}, \min }\left(\bar{N}_{f}\right)=\zeta_{\bar{N}_{f}, 0}=\varepsilon_{f} \bar{N}_{f}+\frac{U-J}{2} \bar{N}_{f}\left(\bar{N}_{f}-1\right) .
$$

In the case of a non-integer occupancy $N_{f}$ of the localized orbitals the minimum energy reads

$$
\begin{aligned}
E_{\mathrm{loc}, \min }\left(\{w\}, N_{f}\right)= & \sum_{i_{\uparrow}=1}^{N_{\text {states }}^{\sigma}\left(\left\lfloor N_{f}\right\rfloor\right)} w_{\left\lfloor N_{f}\right\rfloor, i_{\uparrow} ; 0} \zeta_{\left\lfloor N_{f}\right\rfloor, 0}+\sum_{i_{\uparrow}=1}^{N_{\text {states }}^{\sigma}\left(\left\lceil N_{f}\right\rceil\right)} w_{\left\lceil N_{f}\right\rceil, i_{\uparrow} ; 0} \zeta_{\left\lceil N_{f}\right\rceil, 0} \\
& +\sum_{i_{\downarrow}=1}^{N_{\text {states }}^{\sigma}\left(\left\lfloor N_{f}\right\rfloor\right)} w_{0 ;\left\lfloor N_{f}\right\rfloor, i_{\downarrow}} \zeta_{0,\left\lfloor N_{f}\right\rfloor}+\sum_{i_{\downarrow}=1}^{N_{\text {states }}^{\sigma}\left(\left\lceil N_{f}\right\rceil\right)} w_{0 ;\left\lceil N_{f}\right\rceil, i_{\downarrow}} \zeta_{0,\left\lceil N_{f}\right\rceil} .
\end{aligned}
$$

Again, we have $\zeta_{\left\lfloor N_{f}\right\rfloor, 0}=\zeta_{0,\left\lfloor N_{f}\right\rfloor}$ and $\zeta_{\left\lceil N_{f}\right\rceil, 0}=\zeta_{0,\left\lceil N_{f}\right\rceil}$, which leads to

$$
\begin{aligned}
E_{\mathrm{loc}, \min }\left(\{w\}, N_{f}\right)= & \zeta_{\left\lfloor N_{f}\right\rfloor, 0}\left[\sum_{i_{\uparrow}=1}^{N_{\text {states }}^{\sigma}\left(\left\lfloor N_{f}\right\rfloor\right)} w_{\left\lfloor N_{f}\right\rfloor, i_{\uparrow} ; 0}+\sum_{i_{\downarrow}=1}^{N_{\text {states }}^{\sigma}\left(\left\lfloor N_{f}\right\rfloor\right)} w_{0 ;\left\lfloor N_{f}\right\rfloor, i_{\downarrow}}\right] \\
& +\zeta_{\left\lceil N_{f}\right\rceil, 0}\left[\sum_{i_{\uparrow}=1}^{N_{\text {states }}^{\sigma}\left(\left\lceil N_{f}\right\rceil\right)} w_{\left\lceil N_{f}\right\rceil, i_{\uparrow} ; 0}+\sum_{i_{\downarrow}=1}^{N_{\text {states }}^{\sigma}\left(\left\lceil N_{f}\right\rceil\right)} w_{0 ;\left\lceil N_{f}\right\rceil, i_{\downarrow}}\right] .
\end{aligned}
$$

With the same rationale as in section 5.2.1, we find

$$
\sum_{i_{\uparrow}=1}^{N_{\text {states }}^{\sigma}\left(\left\lfloor N_{f}\right\rfloor\right)} w_{\left\lfloor N_{f}\right\rfloor, i_{\uparrow} ; 0}+\sum_{i_{\downarrow}=1}^{N_{\text {states }}^{\sigma}\left(\left\lfloor N_{f}\right\rfloor\right)} w_{0 ;\left\lfloor N_{f}\right\rfloor, i_{\downarrow}}=n_{f}^{\mathrm{frac}}
$$

and

$$
\sum_{i_{\uparrow}=1}^{N_{\text {states }}^{\sigma}\left(\left\lceil N_{f}\right\rceil\right)} w_{\left\lceil N_{f}\right\rceil, i_{\uparrow} ; 0}+\sum_{i_{\downarrow}=1}^{N_{\text {states }}^{\sigma}\left(\left\lceil N_{f}\right\rceil\right)} w_{0 ;\left\lceil N_{f}\right\rceil, i_{\downarrow}}=1-n_{f}^{\mathrm{frac}},
$$


5 The density of states of localized orbitals with fractional occupancy: study of a model system

so that

$$
\begin{aligned}
E_{\mathrm{loc}, \min }\left(N_{f}\right) & =\left(1-n_{f}^{\mathrm{frac}}\right) \zeta_{\left\lfloor N_{f}\right\rfloor, 0}+n_{f}^{\mathrm{frac}} \zeta_{\left\lceil N_{f}\right\rceil, 0} \\
& =\zeta_{\left\lfloor N_{f}\right\rfloor, 0}+n_{f}^{\mathrm{frac}} \cdot\left[\zeta_{\left\lceil N_{f}\right\rceil, 0}-\zeta_{\left\lfloor N_{f}\right\rfloor, 0}\right] \\
& =\varepsilon_{f} N_{f}+\frac{U-J}{2} N_{f}^{2}-\frac{U-J}{2}\left\lfloor N_{f}\right\rfloor-\frac{U-J}{2}\left(n_{f}^{\mathrm{frac}}\right)^{2} .
\end{aligned}
$$

We see that eq. (5.109) is the same energy curve as in the spinless model, eq. (5.27), however, with the interaction rescaled to $U-J$.

To find the impurity occupancy in the ground state of the model, we minimize the total energy

$$
\begin{aligned}
E_{\mathrm{tot}}\left(N_{f}\right) & =E_{\mathrm{bath}}\left(N_{f}\right)+E_{\mathrm{loc}, \min }\left(N_{f}\right) \\
& =\frac{1}{2 D}\left(N_{\mathrm{tot}}-N_{f}\right)^{2}+E_{\mathrm{loc}, \min }\left(N_{f}\right)
\end{aligned}
$$

with respect to the total occupancy $N_{f}$ of the impurity. For a fractional occupancy, the Fermi level reads (see eqs. (5.32) and (5.33))

$$
\begin{aligned}
E_{\mathrm{F}} & =\zeta_{\left\lceil N_{f}\right\rceil, 0}-\zeta_{\left\lfloor N_{f}\right\rfloor, 0} \\
& =\varepsilon_{f}+(U-J)\left\lfloor N_{f}\right\rfloor .
\end{aligned}
$$

\subsubsection{The impurity spectral function in the many-body ap- proach}

From the previous section it is clear that only certain Slater determinants can have non-zero weights in the energy-minimizing ensemble of the impurity. For an integer occupancy $\bar{N}_{f}$ of the impurity only the weights of Slater determinants whose occupancies satisfy $N_{\sigma}=\bar{N}_{f}, N_{\bar{\sigma}}=0$ can be non-zero. The ground state ensemble should satisfy the maximum entropy principle [144, 145], so that all weights of Slater determinants of the form $N_{\sigma}=\bar{N}_{f}, N_{\bar{\sigma}}=0$ have the same non-zero weight. Similarly, for a fractional occupancy of the impurity only the weights of Slater determinants can be non-zero, which have occupancies $N_{\sigma}=\left\lfloor N_{f}\right\rfloor, N_{\bar{\sigma}}=0$ or $N_{\sigma}=\left\lceil N_{f}\right\rceil, N_{\bar{\sigma}}=0$. Again, the ground state ensemble of the impurity should satisfy the maximum entropy principle, so that all weights associated with Slater determinants of the form $N_{\sigma}=\left\lfloor N_{f}\right\rfloor, N_{\bar{\sigma}}=0$ are non-zero and of equal value, and all weights associated with Slater determinants of the form $N_{\sigma}=\left\lceil N_{f}\right\rceil, N_{\bar{\sigma}}=0$ are non-zero and of equal value. This ensemble is symmetric in the spin and in the impurity orbitals. Hence, we obtain the same spectral function for each spin-orbital $m \sigma$.

Note that we can reuse the result for the spectral function of the spinless model for excitations of the types $\left(N_{\sigma}=\bar{N}_{f}, N_{\bar{\sigma}}=0\right) \rightarrow\left(N_{\sigma}=\bar{N}_{f}+1, N_{\bar{\sigma}}=0\right)$ and $\left(N_{\sigma}=\bar{N}_{f}, N_{\bar{\sigma}}=0\right) \rightarrow\left(N_{\sigma}=\bar{N}_{f}-1, N_{\bar{\sigma}}=0\right)$. However, the peak distance is rescaled to $U-J$ due to the inclusion of Hund's coupling. Furthermore, because there are two spin directions, the weights associated with the individual Slater determinants are divided by 2 . Additional contributions to the spectral 
functions of a spin-orbital $m \sigma$ come from excitations of the type $\left(N_{\sigma}=0, N_{\bar{\sigma}}=\right.$ $\left.\bar{N}_{f}\right) \rightarrow\left(N_{\sigma}=1, N_{\bar{\sigma}}=\bar{N}_{f}\right)$, in which a particle is inserted into the spin-orbital $m \sigma$, which is of the minority spin-direction. In total, for an integer occupancy of the impurity, for an orbital of spin $\uparrow$ we obtain the spectral function,

$$
\begin{aligned}
A_{\mathrm{loc}}\left(\varepsilon, \bar{N}_{f}, \uparrow\right)=\frac{1}{2} & \frac{\bar{N}_{f}}{N_{\mathrm{orb}}} \delta\left(\zeta_{\bar{N}_{f}-1,0}-\zeta_{\bar{N}_{f}, 0}+\varepsilon\right) \\
& +\frac{1}{2} \frac{N_{\mathrm{orb}}-\bar{N}_{f}}{N_{\mathrm{orb}}} \delta\left(\zeta_{\bar{N}_{f}, 0}-\zeta_{\bar{N}_{f}+1,0}+\varepsilon\right) \\
& +\frac{1}{2} \delta\left(\zeta_{0, \bar{N}_{f}}-\zeta_{1, \bar{N}_{f}}+\varepsilon\right) .
\end{aligned}
$$

The first two terms are analogous to those in the spectral function of the spinless model, eq. (5.41). The third term comes from the excitation of a Slater determinant with spin- $\downarrow$ occupancy only, in which a particle is inserted into an orbital of spin $\uparrow$. Similarly, the spectral function for an orbital of spin $\uparrow$ is obtained for a fractional occupancy of the impurity. It reads

$$
\begin{aligned}
A_{\text {loc }}\left(\varepsilon, N_{f}, \uparrow\right)=\frac{\left\lfloor N_{f}\right\rfloor}{N_{\text {orb }}} \frac{1-n_{f}^{\mathrm{frac}}}{2} \delta\left(\zeta_{\left\lfloor N_{f}\right\rfloor-1,0}-\zeta_{\left\lfloor N_{f}\right\rfloor, 0}+\varepsilon\right) \\
+\frac{N_{\text {orb }}-\left\lfloor N_{f}\right\rfloor}{N_{\text {orb }}} \frac{1-n_{f}^{\mathrm{frac}}}{2} \delta\left(\zeta_{\left\lfloor N_{f}\right\rfloor, 0}-\zeta_{\left\lceil N_{f}\right\rceil, 0}+\varepsilon\right) \\
+\frac{\left\lceil N_{f}\right\rceil}{N_{\text {orb }}} \frac{n_{f}^{\mathrm{frac}}}{2} \delta\left(\zeta_{\left\lfloor N_{f}\right\rfloor, 0}-\zeta_{\left\lceil N_{f}\right\rceil, 0}+\varepsilon\right) \\
+\frac{N_{\text {orb }}-\left\lceil N_{f}\right\rceil}{N_{\text {orb }}} \frac{n_{f}^{\mathrm{frac}}}{2} \delta\left(\zeta_{\left\lceil N_{f}\right\rceil, 0}-\zeta_{\left\lceil N_{f}\right\rceil+1,0}+\varepsilon\right) \\
+\frac{1-n_{f}^{\mathrm{frac}}}{2} \delta\left(\zeta_{0,\left\lfloor N_{f}\right\rfloor}-\zeta_{1,\left\lfloor N_{f}\right\rfloor}+\varepsilon\right) \\
+\frac{n_{f}^{\mathrm{frac}}}{2} \delta\left(\zeta_{0,\left\lceil N_{f}\right\rceil}-\zeta_{1,\left\lceil N_{f}\right\rceil}+\varepsilon\right) .
\end{aligned}
$$

Again, the first four terms are analogous to those in the spectral function of the spinless model, eq. (5.48). The last two terms come from insertions of a spin- $\uparrow$ particle into Slater determinants with occupancies $N_{\uparrow}=0, N_{\downarrow}=\left\lfloor N_{f}\right\rfloor$ and $N_{\uparrow}=0, N_{\downarrow}=\left\lceil N_{f}\right\rceil$, respectively. Note that the spectral function of the spin- $\downarrow$ orbitals is identical to those of the spin- $\uparrow$ orbitals. Hence, the spectral functions (5.114) and (5.115) are identical to the average spectral functions over all spin-orbitals for integer and fractional occupancies, respectively.

The spectral function shows the excitation energies associated with particleinsertion and particle-removal excitations of the Slater determinants within the impurity ensemble. In the following we state the excitation energies for excitations of Slater determinants within the impurity ensemble, in which only spin- $\uparrow$ particles are present. Because the Hamiltonian $\hat{H}_{\text {loc }}$ is symmetric in the spin, the excitation energies of Slater determinants within the impurity ensemble, in which only spin- $\downarrow$ particles are present, can be obtained analogously. 
5 The density of states of localized orbitals with fractional occupancy: study of a model system

For the insertion of an electron of the spin- $\uparrow$ direction we find the excitation energy

$$
\zeta_{\bar{N}_{\uparrow}+1, \bar{N}_{\downarrow}}-\zeta_{\bar{N}_{\uparrow}, \bar{N}_{\downarrow}}=\varepsilon_{f}+(U-J) \bar{N}_{\uparrow}+U \bar{N}_{\downarrow} .
$$

The removal of an electron from the spin- $\uparrow$ direction is associated with the excitation energy

$$
\zeta_{\bar{N}_{\uparrow}, \bar{N}_{\downarrow}}-\zeta_{\bar{N}_{\uparrow}-1, \bar{N}_{\downarrow}}=\varepsilon_{f}+(U-J)\left(\bar{N}_{\uparrow}-1\right)+U \bar{N}_{\downarrow},
$$

Hence, in the spectral function, the energy separation of the peaks of particleinsertion and particle-removal excitations for spin- $\uparrow$ particles is

$$
\left(\zeta_{\bar{N}_{\uparrow}+1, \bar{N}_{\downarrow}}-\zeta_{\bar{N}_{\uparrow}, \bar{N}_{\downarrow}}\right)-\left(\zeta_{\bar{N}_{\uparrow}, \bar{N}_{\downarrow}}-\zeta_{\bar{N}_{\uparrow}-1, \bar{N}_{\downarrow}}\right)=U-J .
$$

Moreover, the energy difference between the insertion of a particle with spin $\uparrow$ and the insertion of a particle with spin $\downarrow$ is

$$
\left(\zeta_{\bar{N}_{\uparrow}, \bar{N}_{\downarrow}+1}-\zeta_{\bar{N}_{\uparrow}, \bar{N}_{\downarrow}}\right)-\left(\zeta_{\bar{N}_{\uparrow}+1, \bar{N}_{\downarrow}}-\zeta_{\bar{N}_{\uparrow}, \bar{N}_{\downarrow}}\right)=J\left(\bar{N}_{\uparrow}-\bar{N}_{\downarrow}\right) .
$$

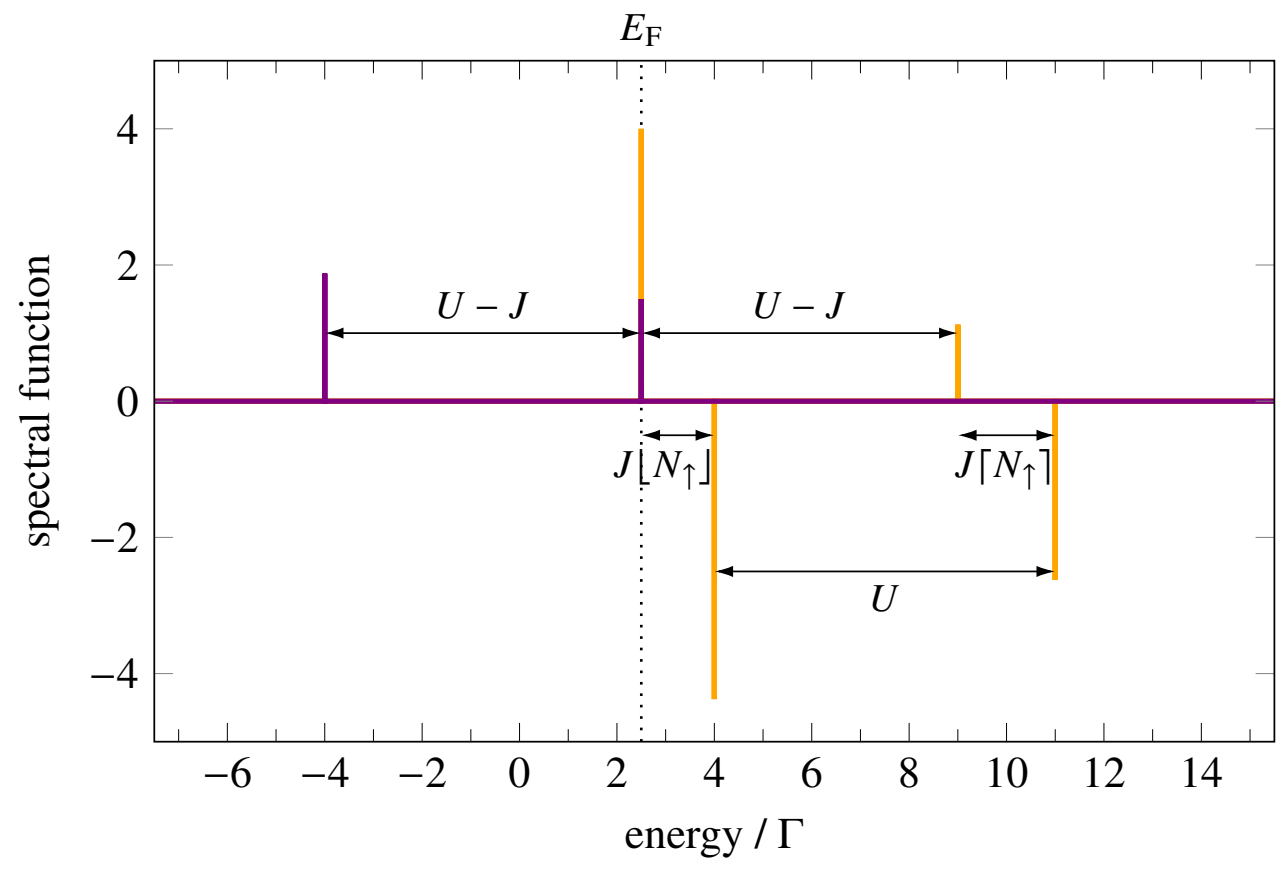

Figure 5.10: Example for the ground state spectral function $2 N_{\mathrm{orb}} A_{\mathrm{loc}}\left(\varepsilon, N_{f}, \uparrow\right)$ (eq. (5.115)) of the localized orbitals in the system with a Hund's coupling between the localized orbitals. Model parameters: $\varepsilon_{f}=$ $-17 \Gamma, U=7 \Gamma, J=0.5 \Gamma, D=0.25 / \Gamma, N_{\text {tot }}=4, N_{\text {orb }}=7$. The total energy is minimized at an occupancy $N_{f}=3.375$. Purple: occupied states, orange: unoccupied states. Positive (negative) values: contributions from excitations in which a particle of the majority (minority) spin direction is inserted into or removed from the impurity. 
We plot an example of the spectral function (5.115) in fig. 5.10. In the upper half of the plot, we show the part of the spectral function that originates from the first four terms of eq. (5.115). The contributions of the last two terms of eq. (5.115) are plotted in the lower half of fig. 5.10. This is not to be confused with a splitting of the spectral function into contributions of the two spin directions.

\subsection{Hartree-Fock approach to the spin-resolved model}

In the following we discuss the treatment of the spin-resolved model Hamiltonian in the Hartree-Fock scheme. We show that a ferromagnetic solution gives the same minimum of the total energy at the same ground state occupancy of the impurity as in the many-particle approach. Moreover, we determine the ground state ensemble of the impurity and calculate the spectral function. Different excitation energies of the Slater determinants that contribute to the ground state ensemble can be determined with the Hamiltonian of the effective non-interacting system of the Hartree-Fock approximation and with the interacting Hamiltonian of the impurity. This gives two possibilities to determine a spectral function from the ground state ensemble. Both possibilities are discussed and the spectral functions of the Hartree-Fock approach and from the many-particle treatment are compared in section 5.8.

\subsubsection{The ground state total energy of the spin-resolved model in the Hartree-Fock approximation}

First, we determine the effective potential part of the impurity Hamiltonian in the Hartree-Fock approximation, consisting of the Hartree and exchange potentials. For this purpose, we insert the elements (5.91) of the simplified interaction tensor into the effective potential part of the general effective noninteracting Hamiltonian (3.79),

$$
\begin{aligned}
\hat{H}_{\mathrm{loc}, \mathrm{int}}^{\mathrm{HF}}= & \sum_{m m^{\prime} m^{\prime \prime} m^{\prime \prime \prime}} \sum_{\sigma \sigma^{\prime} \sigma^{\prime \prime} \sigma^{\prime \prime \prime}}\left(U_{m^{\prime \prime \prime} \sigma^{\prime \prime \prime} m \sigma m^{\prime \prime} \sigma^{\prime \prime} m^{\prime} \sigma^{\prime}}-\eta U_{m^{\prime \prime \prime} \sigma^{\prime \prime \prime} m \sigma m^{\prime} \sigma^{\prime} m^{\prime \prime} \sigma^{\prime \prime}}\right) \\
& \times\left\langle\hat{f}_{m^{\prime \prime \prime} \sigma^{\prime \prime \prime}} \hat{f}_{m^{\prime \prime} \sigma^{\prime \prime}}\right\rangle_{\mathrm{HF}} \hat{f}_{m \sigma}^{\dagger} \hat{f}_{m^{\prime} \sigma^{\prime}} \\
= & U \sum_{m m^{\prime}} \sum_{\sigma \sigma^{\prime}}\left\langle\hat{f}_{m^{\prime} \sigma^{\prime}}^{\dagger} \hat{f}_{m^{\prime} \sigma^{\prime}}\right\rangle_{\mathrm{HF}} \hat{f}_{m \sigma}^{\dagger} \hat{f}_{m \sigma}+J \sum_{\substack{m m^{\prime} \\
m \neq m^{\prime}}} \sum_{\sigma}\left\langle\hat{f}_{m^{\prime} \sigma}^{\dagger} \hat{f}_{m \sigma}\right\rangle_{\mathrm{HF}} \hat{f}_{m \sigma}^{\dagger} \hat{f}_{m^{\prime} \sigma} \\
& -\eta U \sum_{m m^{\prime}} \sum_{\sigma \sigma^{\prime}}\left\langle\hat{f}_{m^{\prime} \sigma^{\prime}}^{\dagger} \hat{f}_{m \sigma}\right\rangle_{\mathrm{HF}} \hat{f}_{m \sigma}^{\dagger} \hat{f}_{m^{\prime} \sigma^{\prime}} \\
& -\eta J \sum_{\substack{m m^{\prime} \\
m \neq m^{\prime}}} \sum_{\sigma}\left\langle\hat{f}_{m^{\prime} \sigma}^{\dagger} \hat{f}_{m^{\prime} \sigma}\right\rangle_{\mathrm{HF}} \hat{f}_{m \sigma}^{\dagger} \hat{f}_{m \sigma} .
\end{aligned}
$$

Like in the Hartree-Fock treatment of the spinless model we introduced a scaling factor $\eta$ for the exchange potential in analogy to the exchange mixing 
5 The density of states of localized orbitals with fractional occupancy: study of a model system

factor in the PBE0r hybrid functional. The Hartree energy is

$$
\begin{aligned}
E_{\mathrm{H}}=\frac{U}{2} & \sum_{m m^{\prime} \sigma \sigma^{\prime}} \sum_{\sigma_{m^{\prime} \sigma^{\prime}}}\left\langle\hat{f}_{m^{\prime} \sigma^{\prime}}^{\dagger}\right\rangle_{\mathrm{HF}}\left\langle\hat{f}_{m \sigma}^{\dagger} \hat{f}_{m \sigma}\right\rangle_{\mathrm{HF}} \\
& +\frac{J}{2} \sum_{\substack{m m^{\prime} \\
m \neq m^{\prime}}} \sum_{\sigma}\left\langle\hat{f}_{m^{\prime} \sigma}^{\dagger} \hat{f}_{m \sigma}\right\rangle_{\mathrm{HF}}\left\langle\hat{f}_{m \sigma}^{\dagger} \hat{f}_{m^{\prime} \sigma}\right\rangle_{\mathrm{HF}}
\end{aligned}
$$

and the exchange energy reads

$$
\begin{aligned}
E_{\mathrm{X}}=- & \frac{U}{2} \sum_{m m^{\prime}} \sum_{\sigma \sigma^{\prime}}\left\langle\hat{f}_{m^{\prime} \sigma^{\prime}}^{\dagger} \hat{f}_{m \sigma}\right\rangle_{\mathrm{HF}}\left\langle\hat{f}_{m \sigma}^{\dagger} \hat{f}_{m^{\prime} \sigma^{\prime}}\right\rangle_{\mathrm{HF}} \\
& -\frac{J}{2} \sum_{\substack{m m^{\prime} \\
m \neq m^{\prime}}} \sum_{\sigma}\left\langle\hat{f}_{m^{\prime} \sigma}^{\dagger} \hat{f}_{m^{\prime} \sigma}\right\rangle_{\mathrm{HF}}\left\langle\hat{f}_{m \sigma}^{\dagger} \hat{f}_{m \sigma}\right\rangle_{\mathrm{HF}} .
\end{aligned}
$$

For the interaction energy of the localized orbitals in the Hartree-Fock approximation, we find

$$
\begin{aligned}
& E_{\mathrm{loc}, \text { int }}^{\mathrm{HF}}=\left\langle\hat{H}_{\mathrm{loc}, \mathrm{int}}^{\mathrm{HF}}\right\rangle_{\mathrm{HF}}-\left(E_{\mathrm{H}}+\eta E_{\mathrm{X}}\right) \\
&=\frac{U}{2}\left[\sum_{m \sigma}\left\langle\hat{f}_{m \sigma}^{\dagger} \hat{f}_{m \sigma}\right\rangle_{\mathrm{HF}}\right]\left[\sum_{m^{\prime} \sigma^{\prime}}\left\langle\hat{f}_{m^{\prime} \sigma^{\prime}}^{\dagger} \hat{f}_{m^{\prime} \sigma^{\prime}}\right\rangle_{\mathrm{HF}}\right] \\
&-\frac{\eta J}{2} \sum_{\sigma} \sum_{\substack{m m^{\prime} \\
m \neq m^{\prime}}}\left\langle\hat{f}_{m \sigma}^{\dagger} \hat{f}_{m \sigma}\right\rangle_{\mathrm{HF}}\left\langle\hat{f}_{m^{\prime} \sigma}^{\dagger} \hat{f}_{m^{\prime} \sigma}\right\rangle_{\mathrm{HF}} \\
&-\frac{\eta U}{2} \sum_{m m^{\prime}} \sum_{\sigma \sigma^{\prime}}\left\langle\hat{f}_{m \sigma}^{\dagger} \hat{f}_{m^{\prime} \sigma^{\prime}}\right\rangle_{\mathrm{HF}}\left\langle\hat{f}_{m^{\prime} \sigma^{\prime}}^{\dagger} \hat{f}_{m \sigma}\right\rangle_{\mathrm{HF}} \\
&+\frac{J}{2} \sum_{\sigma} \sum_{\substack{m m^{\prime} \\
m \neq m^{\prime}}}\left\langle\hat{f}_{m \sigma}^{\dagger} \hat{f}_{m^{\prime} \sigma}\right\rangle_{\mathrm{HF}}\left\langle\hat{f}_{m^{\prime} \sigma}^{\dagger} \hat{f}_{m \sigma}\right\rangle_{\mathrm{HF}} .
\end{aligned}
$$

The complete effective non-interacting Hamiltonian for the impurity in the Hartree-Fock approximation reads

$$
\hat{H}_{\mathrm{loc}}^{\mathrm{HF}}=\varepsilon_{f} \sum_{m \sigma} \hat{n}_{m \sigma}+\hat{H}_{\mathrm{loc}, \mathrm{int}}^{\mathrm{HF}}
$$

and the Hartree-Fock approximation to the total energy of the interacting impurity is

$$
E_{\mathrm{loc}}^{\mathrm{HF}}=\varepsilon_{f} \sum_{m \sigma}\left\langle\hat{n}_{m \sigma}\right\rangle_{\mathrm{HF}}+E_{\mathrm{loc}, \mathrm{int}}^{\mathrm{HF}} .
$$

If the density matrix is diagonal in the spin-orbital basis,

$$
\left\langle\hat{f}_{m \sigma}^{\dagger} \hat{f}_{m^{\prime} \sigma^{\prime}}\right\rangle_{\mathrm{HF}}=\left\langle\hat{n}_{m \sigma}\right\rangle_{\mathrm{HF}} \delta_{\sigma \sigma^{\prime}} \delta_{m m^{\prime}},
$$

the density matrix and the effective non-interacting Hamiltonian (5.127) are simultaneously diagonal and the self-consistency condition of the Hartree- 
Fock approximation is satisfied. This leads to the ferromagnetic solution that is discussed in the following. The total energy of the impurity in the Hartree-Fock approximation becomes

$$
\begin{aligned}
E_{\mathrm{loc}}^{\mathrm{HF}} & =\varepsilon_{f} N_{f}+\frac{U}{2} N_{f}^{2}-\frac{\eta J}{2} \sum_{\sigma} N_{\sigma}^{2}-\frac{\eta(U-J)}{2} \sum_{m \sigma}\left\langle\hat{n}_{m \sigma}\right\rangle_{\mathrm{HF}}^{2} \\
& =\varepsilon_{f} N_{f}+\frac{U-\eta J}{2} N_{f}^{2}+\eta J N_{\uparrow} N_{\downarrow}-\frac{\eta(U-J)}{2} \sum_{m \sigma}\left\langle\hat{n}_{m \sigma}\right\rangle_{\mathrm{HF}}^{2}
\end{aligned}
$$

The term $\eta J N_{\uparrow} N_{\downarrow}$ couples the occupancies of both spin directions and increases the total energy of the impurity as soon as particles of both spin directions reside on the impurity. For less than half-filling, which is the case in the $f$ shell of the light rare-earth hexaborides, eq. (5.131) can only be minimal if all particles on the impurity have the same spin. For an integer occupancy where $N_{\uparrow}=\bar{N}_{f}, N_{\downarrow}=0$, the minimum total energy of the localized orbitals is

$$
E_{\mathrm{loc}, \min }^{\mathrm{HF}}\left(\bar{N}_{\uparrow}=\bar{N}_{f}, \bar{N}_{\downarrow}=0\right)=\varepsilon_{f} \bar{N}_{f}+\frac{U-\eta J}{2} \bar{N}_{f}^{2}-\frac{\eta(U-J)}{2} \bar{N}_{f} .
$$

In the case of a fractional ground state occupancy $N_{\uparrow}=N_{f}, N_{\downarrow}=0$ of the localized orbitals we obtain the minimum total energy of the localized orbitals if only one orbital has a fractional occupancy $n_{f}^{\text {frac }}$ while $\left\lfloor N_{f}\right\rfloor$ orbitals have occupancy 1 ,

$$
\begin{aligned}
E_{\mathrm{loc}, \min }^{\mathrm{HF}}\left(N_{\uparrow}=N_{f}, N_{\downarrow}=0\right)=\varepsilon_{f} & N_{f}+\frac{U-\eta J}{2} N_{f}^{2}-\frac{\eta(U-J)}{2}\left\lfloor N_{f}\right\rfloor \\
& -\frac{\eta(U-J)}{2}\left(n_{f}^{\mathrm{frac}}\right)^{2} .
\end{aligned}
$$

Again, the ground state total energy of the complete model system and the ground state occupancy of the impurity are obtained from a minimization of the total energy

$$
E_{\mathrm{tot}}^{\mathrm{HF}}\left(N_{f}\right)=E_{\mathrm{bath}}\left(N_{f}\right)+E_{\mathrm{loc}, \min }^{\mathrm{HF}}\left(N_{\uparrow}=N_{f}, N_{\downarrow}=0\right)
$$

with respect to the occupancy number $N_{f}$.

Note that, for $\eta=1$, the expressions (5.132) and (5.133) for the minimum total energy of the impurity are identical to eqs. (5.102) and (5.109) of the many-particle approach for an integer occupancy and a fractional occupancy of the impurity, respectively. Because the minimum energy in the Hartree-Fock approximation cannot be smaller than that of the many-particle calculation, the ferromagnetic solution with the density matrix (5.129) leads to a global minimum of the total energy. 


\subsubsection{The ground state ensemble of the impurity in the Hartree- Fock approximation}

The total occupancies of the two spin directions, $N_{\uparrow}$ and $N_{\downarrow}$, in the ground state are obtained by averaging over the ground state ensemble of the impurity. The Hund's coupling term $J N_{\uparrow} N_{\downarrow}$ in eq. (5.131) suppresses an occupancy of both spin directions in the ground state. Hence, in the ground state, the spin symmetry is broken and we have an occupied spin direction $\sigma$ and an unoccupied spin direction $\bar{\sigma}$. Only Slater determinants in which all particles are of the majority spin direction $\sigma$ can contribute to the ground state ensemble.

In the following we assume that the impurity in the ground state is occupied by spin- $\uparrow$ particles only. For an integer ground state occupancy $\bar{N}_{f}^{\mathrm{GS}}$ of the impurity, $\bar{N}_{f}^{\mathrm{GS}}$ orbitals of spin $\uparrow$ have occupancy 1 . This is only possible if the ground state ensemble of the impurity reduces to a single Slater determinant, in which the selected orbitals are occupied. The symmetry of the localized orbitals is broken. In the case of a fractional ground state occupancy $N_{f}^{\mathrm{GS}}$ of the impurity, we have $\left\lfloor N_{f}^{\mathrm{GS}}\right\rfloor$ orbitals of spin $\uparrow$ with occupancy 1 and one orbital with a fractional occupancy $n_{f}^{\text {frac }}$. This can be achieved only by an ensemble in which one Slater determinant with $\left\lceil N_{f}\right\rceil$ particles and weight $n_{f}^{\text {frac }}$ contributes, as well as one Slater determinant with $\left\lfloor N_{f}\right\rfloor$ particles and weight $1-n_{f}^{\text {frac }}$. Moreover, the same three orbitals have to be occupied in both Slater determinants and one localized orbital is selected for fractional occupancy. Again, the symmetry in the localized orbitals is broken. For more details, we refer to section 5.3.2, where analogous ground state ensembles for the spinless model are discussed.

Again, we have two possibilities to obtain a spectral function in the HartreeFock approximation, see also section 5.3.2. First, we determine the spectral function in which the excitation energies of the states of the impurity ground state ensemble are calculated with the effective non-interacting Hamiltonian $\hat{H}_{\mathrm{loc}}^{\mathrm{HF}}$. Second, we use the excitation energies of the states of the impurity ground state ensemble computed with the interacting Hamiltonian $\hat{H}_{\mathrm{loc}}$ to determine the spectral function.

\subsubsection{The impurity spectral function with respect to $\hat{H}_{\text {loc }}^{\mathrm{HF}}$}

In the spin-orbital basis the effective non-interacting impurity Hamiltonian of the Hartree-Fock approach becomes diagonal if the density matrix is diagonal, and reads

$$
\hat{H}_{\mathrm{loc}}^{\mathrm{HF}}=\sum_{m \sigma} \xi_{m \sigma} \hat{n}_{m \sigma} .
$$

The energy levels $\xi_{m \sigma}$ of the Hartree-Fock one-particle basis states are determined via the derivative of the total energy (5.131) with respect to the 
spin-orbital occupancy number:

$$
\xi_{m \sigma}=\frac{\partial E_{\mathrm{loc}}^{\mathrm{HF}}}{\partial\left\langle\hat{n}_{m \sigma}\right\rangle_{\mathrm{HF}}}=\varepsilon_{f}+(U-\eta J) N_{f}+\eta J N_{\bar{\sigma}}-\eta(U-J)\left\langle\hat{n}_{m \sigma}\right\rangle_{\mathrm{HF}}
$$

Hence, for the ground state ensemble where $N_{\uparrow}=N_{f}$ and $N_{\downarrow}=0$, we obtain the one-particle energies

$$
\begin{aligned}
& \xi_{\text {occ }, \uparrow}\left(N_{\uparrow}=N_{f}, N_{\downarrow}=0\right)=\varepsilon_{f}+(U-\eta J) N_{\uparrow}-\eta(U-J), \\
& \xi_{\text {unocc }, \uparrow}\left(N_{\uparrow}=N_{f}, N_{\downarrow}=0\right)=\varepsilon_{f}+(U-\eta J) N_{\uparrow}, \\
& \xi_{\text {frac }, \uparrow}\left(N_{\uparrow}=N_{f}, N_{\downarrow}=0\right)=\varepsilon_{f}+(U-\eta J) N_{\uparrow}-\eta(U-J) n_{\uparrow}^{\text {frac }},
\end{aligned}
$$

for the occupied, unoccupied, and fractionally occupied orbitals of the spin- $\uparrow$ direction, respectively. Moreover, the energy of the unoccupied states in the spin- $\downarrow$ direction is

$$
\xi_{\text {unocc }, \downarrow}\left(N_{\uparrow}=N_{f}, N_{\downarrow}=0\right)=\varepsilon_{f}+U N_{\uparrow} .
$$

The separation of the fully occupied and fully unoccupied states for spin $\uparrow$ reads

$$
\xi_{\text {unocc }, \uparrow}-\xi_{\text {occ }, \uparrow}=\eta(U-J) .
$$

Moreover, the energy separation between the completely unoccupied states of both spin directions is

$$
\xi_{\text {unocc }, \downarrow}-\xi_{\text {unocc }, \uparrow}=\eta J\left(N_{\uparrow}-N_{\downarrow}\right)=\eta J N_{\uparrow} .
$$

Note that this result is the same as eq. (5.119) of the many-body approach only for an integer occupancy of the localized orbitals.

The Fermi level in the case of a fractional occupancy of the impurity is obtained from the minimum condition

$$
\frac{\partial E_{\mathrm{tot}}^{\mathrm{HF}}}{\partial n_{f}^{\mathrm{frac}}}=-\frac{1}{D}\left(N_{\mathrm{tot}}-N_{f}\right)+\varepsilon_{f}+(U-\eta J) N_{\uparrow}-\eta(U-J) n_{f}^{\mathrm{frac}} \stackrel{!}{=} 0 .
$$

With eqs. (5.5) and (5.139) we find

$$
E_{\mathrm{F}}=\xi_{\text {frac }, \uparrow} \cdot
$$

Hence, for a fractional occupancy of the impurity, the fractionally occupied orbital is pinned to the Fermi level.

We obtain the spectral function of the impurity by averaging over the spectral functions of all localized orbitals. For an integer occupancy of the impurity, it reads

$$
\begin{aligned}
A_{\mathrm{loc}}^{\mathrm{HF}}\left(\varepsilon, \bar{N}_{f}\right)= & \frac{\bar{N}_{f}}{2 N_{\mathrm{orb}}} \delta\left(\varepsilon-\xi_{\mathrm{occ}, \uparrow}\right)+\frac{N_{\text {orb }}-\bar{N}_{f}}{2 N_{\text {orb }}} \delta\left(\varepsilon-\xi_{\text {unocc }, \uparrow}\right) \\
& +\frac{1}{2} \delta\left(\varepsilon-\xi_{\text {unocc }, \downarrow}\right)
\end{aligned}
$$


5 The density of states of localized orbitals with fractional occupancy: study of a model system

and for a fractional occupancy of the impurity it is

$$
\begin{aligned}
A_{\mathrm{loc}}^{\mathrm{HF}}\left(\varepsilon, N_{f}\right)=\frac{\left\lfloor N_{f}\right\rfloor}{2 N_{\mathrm{orb}}} \delta\left(\varepsilon-\xi_{\mathrm{occ}, \uparrow}\right)+\frac{1-n_{f}^{\mathrm{frac}}}{2 N_{\mathrm{orb}}} \delta\left(\varepsilon-\xi_{\text {frac }, \uparrow}\right) \\
+\frac{n_{f}^{\mathrm{frac}}}{2 N_{\mathrm{orb}}} \delta\left(\varepsilon-\xi_{\text {frac }, \uparrow}\right)+\frac{N_{\mathrm{orb}}-\left\lceil N_{f}\right\rceil}{2 N_{\text {orb }}} \delta\left(\varepsilon-\xi_{\text {unocc }, \uparrow}\right) \\
+\frac{1}{2} \delta\left(\varepsilon-\xi_{\text {unocc }, \downarrow}\right) .
\end{aligned}
$$

An example plot of the spectral function for the case of a fractional occupancy of the impurity is depicted in fig. 5.11. The spectral function of the many body approach is added to the plot to facilitate a comparison.

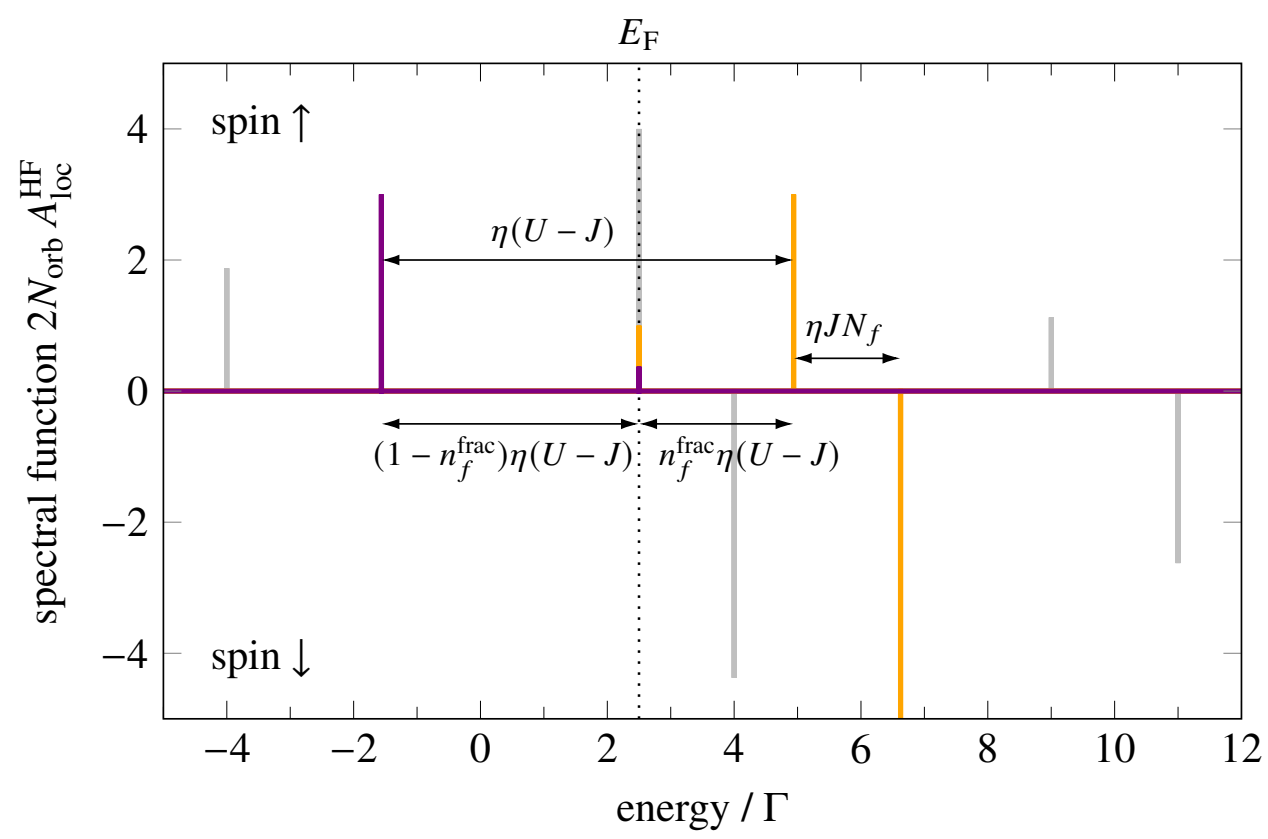

Figure 5.11: Example for the spectral function $2 N_{\mathrm{orb}} A_{\mathrm{loc}}^{\mathrm{HF}}\left(\varepsilon, N_{f}\right)$ of the impurity in the spin-resolved model system with energy levels as calculated from the effective non-interacting Hamiltonian $\hat{H}_{\mathrm{loc}}^{\mathrm{HF}}$, eq. (5.135). The impurity is fractionally occupied. Model parameters: $\varepsilon_{f}=-17 \Gamma, U=7 \Gamma, J=0.5 \Gamma, \eta=1, D=0.25 / \Gamma$, $N_{\text {tot }}=4, N_{\text {orb }}=7$. The total energy is minimized at an occupancy $N_{f}^{\mathrm{GS}}=3.375$ of the impurity. Purple: occupied states, orange: unoccupied states. Positive values: spin- $\uparrow$ orbitals, negative values: spin- $\downarrow$ orbitals. For comparison the gray $\delta$ peaks show the one-particle spectral function of the model from the many-particle approach for the same set of parameters.

\subsubsection{The impurity spectral function with respect to $\hat{H}_{\text {loc }}$}

Now we turn to the second possibility to calculate the spectral function from the ground state ensemble of the impurity in the Hartree-Fock approximation. Here 
we employ the interacting Hamiltonian $\hat{H}_{\text {loc }}$ to obtain the excitation energies of particle-insertion and particle-removal excitations of the Slater determinants within the ground state ensemble of the impurity. The Slater determinants that contribute to the ground state ensemble and the associated excited state Slater determinants are eigenstates of $\hat{H}_{\text {loc }}$. Hence, the excitation energies are differences of the eigenvalues (5.100) for adjacent particle numbers, see also section 5.6.2.

The spin symmetry and the orbital symmetry are broken in the ground state ensemble of the impurity in the Hartree-Fock approximation. Hence, the spectral functions of the individual orbitals differ, based on their occupancy. This is in contrast to the spectral functions of the individual spin-orbitals in the many-body approach. In the following we list the spectral functions of the individual localized orbitals in the Hartree-Fock approximation for excitation energies computed with the interacting Hamiltonian $\hat{H}_{\text {loc }}$. We use the symbol $B_{\mathrm{loc}}^{\mathrm{HF}}$ to distinguish this spectral function from the spectral function $A_{\mathrm{loc}}$ of the many-particle treatment and from the spectral function $A_{\mathrm{loc}}^{\mathrm{HF}}$ of the effective non-interacting system of the Hartree-Fock approach.

For an integer occupancy of the impurity we find the spectral functions

$$
B_{\mathrm{loc}, \mathrm{occ}}^{\mathrm{HF}}\left(\varepsilon, \bar{N}_{f}, \uparrow\right)=\delta\left(\zeta_{\bar{N}_{f}-1,0}-\zeta_{\bar{N}_{f}, 0}+\varepsilon\right)
$$

for an occupied orbital in the spin- $\uparrow$ direction,

$$
B_{\text {loc,unocc }}^{\mathrm{HF}}\left(\varepsilon, \bar{N}_{f}, \uparrow\right)=\delta\left(\zeta_{\bar{N}_{f}, 0}-\zeta_{\bar{N}_{f}+1,0}+\varepsilon\right)
$$

for an unoccupied orbital in the spin- $\uparrow$ direction, and

$$
B_{\text {loc,unocc }}^{\mathrm{HF}}\left(\varepsilon, \bar{N}_{f}, \downarrow\right)=\delta\left(\zeta_{\bar{N}_{f}, 0}-\zeta_{\bar{N}_{f}, 1}+\varepsilon\right)
$$

for an unoccupied orbital in the spin- $\downarrow$ direction. In the case of a fractional ground state occupancy of the impurity, we find the spectral functions

$$
\begin{aligned}
B_{\mathrm{loc}, \mathrm{occ}}^{\mathrm{HF}}\left(\varepsilon, N_{f}, \uparrow\right)=(1 & \left.-n_{f}^{\mathrm{frac}}\right) \delta\left(\zeta_{\left\lfloor N_{f}\right\rfloor-1,0}-\zeta_{\left\lfloor N_{f}\right\rfloor, 0}+\varepsilon\right) \\
& +n_{f}^{\mathrm{frac}} \delta\left(\zeta_{\left\lfloor N_{f}\right\rfloor, 0}-\zeta_{\left\lceil N_{f}\right\rceil, 0}+\varepsilon\right)
\end{aligned}
$$

for an occupied orbital in the spin- $\uparrow$ direction,

$$
\begin{aligned}
B_{\text {loc,unocc }}^{\mathrm{HF}}\left(\varepsilon, N_{f}, \uparrow\right)=(1 & \left.-n_{f}^{\mathrm{frac}}\right) \delta\left(\zeta_{\left\lfloor N_{f}\right\rfloor, 0}-\zeta_{\left\lceil N_{f}\right\rceil, 0}+\varepsilon\right) \\
& +n_{f}^{\mathrm{frac}} \delta\left(\zeta_{\left\lceil N_{f}\right\rceil, 0}-\zeta_{\left\lceil N_{f}\right\rceil+1,0}+\varepsilon\right)
\end{aligned}
$$

for an unoccupied orbital in the spin- $\uparrow$ direction,

$$
\begin{aligned}
B_{\mathrm{loc}, \text { frac }}^{\mathrm{HF}}\left(\varepsilon, N_{f}, \uparrow\right)=(1 & \left.-n_{f}^{\mathrm{frac}}\right) \delta\left(\zeta_{\left\lfloor N_{f}\right\rfloor, 0}-\zeta_{\left\lceil N_{f}\right\rceil, 0}+\varepsilon\right) \\
& +n_{f}^{\mathrm{frac}} \delta\left(\zeta_{\left\lfloor N_{f}\right\rfloor, 0}-\zeta_{\left\lceil N_{f}\right\rceil, 0}+\varepsilon\right)
\end{aligned}
$$


5 The density of states of localized orbitals with fractional occupancy: study of a model system

for the fractionally occupied orbital in the spin- $\uparrow$ direction, and

$$
\begin{aligned}
B_{\text {loc }, \text { unocc }}^{\mathrm{HF}}\left(\varepsilon, N_{f}, \downarrow\right)=(1 & \left.-n_{f}^{\mathrm{frac}}\right) \delta\left(\zeta_{\left\lfloor N_{f}\right\rfloor, 0}-\zeta_{\left\lfloor N_{f}\right\rfloor, 1}+\varepsilon\right) \\
& +n_{f}^{\mathrm{frac}} \delta\left(\zeta_{\left\lceil N_{f}\right\rceil, 0}-\zeta_{\left\lceil N_{f}\right\rceil, 1}+\varepsilon\right)
\end{aligned}
$$

for an unoccupied orbital in the spin- $\downarrow$ direction. Here each Slater determinant has two contributions coming from the two Slater determinants with non-zero weights $\left(1-n_{f}^{\text {frac }}\right)$ and $n_{f}^{\text {frac }}$ in the ground state ensemble of the impurity. We can calculate the average over the spectral functions of the individual orbitals for each spin direction to obtain a spin-resolved spectral function. For an integer occupancy of the impurity we find

$$
\begin{aligned}
B_{\mathrm{loc}}^{\mathrm{HF}}\left(\varepsilon, \bar{N}_{f}, \uparrow\right)= & \frac{\bar{N}_{f}}{N_{\mathrm{orb}}} \delta\left(\zeta_{\bar{N}_{f}-1,0}-\zeta_{\bar{N}_{f}, 0}+\varepsilon\right) \\
& +\frac{N_{\mathrm{orb}}-\bar{N}_{f}}{N_{\mathrm{orb}}} \delta\left(\zeta_{\bar{N}_{f}, 0}-\zeta_{\bar{N}_{f}+1,0}+\varepsilon\right)
\end{aligned}
$$

and

$$
B_{\mathrm{loc}}^{\mathrm{HF}}\left(\varepsilon, \bar{N}_{f}, \downarrow\right)=\delta\left(\zeta_{\bar{N}_{f}, 0}-\zeta_{\bar{N}_{f}, 1}+\varepsilon\right)
$$

for the spectral functions of the spin- $\uparrow$ and spin- $\downarrow$ directions, respectively. In the case of a fractional occupancy of the impurity we have

$$
\begin{aligned}
B_{\mathrm{loc}}^{\mathrm{HF}}\left(\varepsilon, N_{f}, \uparrow\right)=(1 & \left.-n_{f}^{\mathrm{frac}}\right) \frac{\left\lfloor N_{f}\right\rfloor}{N_{\text {orb }}} \delta\left(\zeta_{\left\lfloor N_{f}\right\rfloor-1,0}-\zeta_{\left\lfloor N_{f}\right\rfloor, 0}+\varepsilon\right) \\
& +n_{f}^{\mathrm{frac}} \frac{\left\lceil N_{f}\right\rceil}{N_{\text {orb }}} \delta\left(\zeta_{\left\lfloor N_{f}\right\rfloor, 0}-\zeta_{\left\lceil N_{f}\right\rceil, 0}+\varepsilon\right) \\
& +\left(1-n_{f}^{\mathrm{frac}}\right) \frac{N_{\mathrm{orb}}-\left\lfloor N_{f}\right\rfloor}{N_{\text {orb }}} \delta\left(\zeta_{\left\lfloor N_{f}\right\rfloor, 0}-\zeta_{\left\lceil N_{f}\right\rceil, 0}+\varepsilon\right) \\
& +n_{f}^{\text {frac }} \frac{N_{\text {orb }}-\left\lceil N_{f}\right\rceil}{N_{\text {orb }}} \delta\left(\zeta_{\left\lceil N_{f}\right\rceil, 0}-\zeta_{\left\lceil N_{f}\right\rceil+1,0}+\varepsilon\right)
\end{aligned}
$$

and

$$
\begin{aligned}
B_{\mathrm{loc}}^{\mathrm{HF}}\left(\varepsilon, N_{f}, \downarrow\right)=(1 & \left.-n_{f}^{\mathrm{frac}}\right) \delta\left(\zeta_{\left\lfloor N_{f}\right\rfloor, 0}-\zeta_{\left\lfloor N_{f}\right\rfloor, 1}+\varepsilon\right) \\
& +n_{f}^{\mathrm{frac}} \delta\left(\zeta_{\left\lceil N_{f}\right\rceil, 0}-\zeta_{\left\lceil N_{f}\right\rceil, 1}+\varepsilon\right)
\end{aligned}
$$

for the spectral functions of the spin- $\uparrow$ and spin- $\downarrow$ directions, respectively. As an example plot for the spectral functions $B_{\mathrm{loc}}^{\mathrm{HF}}\left(\varepsilon, N_{f}, \uparrow\right)$ and $B_{\mathrm{loc}}^{\mathrm{HF}}\left(\varepsilon, N_{f}, \downarrow\right)$ for the case of a fractional occupancy of the impurity, we can again consider fig. 5.10. However, because of the broken symmetry in the Hartree-Fock approximation the interpretation of the plot is different than in the case of the spectral function from the many-particle treatment of the model. At the same model parameters, a plot of $B_{\mathrm{loc}}^{\mathrm{HF}}\left(\varepsilon, N_{f}, \uparrow\right)$ is identical to the spectral function in the upper half of 
fig. 5.10. Moreover, a plot of $B_{\mathrm{loc}}^{\mathrm{HF}}\left(\varepsilon, N_{f}, \downarrow\right)$ is identical to the spectral function in the lower half of fig. 5.10.

\subsection{Discussion of the spin-resolved model}

In section 5.5 we extend the spinless model to a spin-resolved model. We include a Hund's coupling term, which favors the occupancy of only one spin direction in accordance with Hund's rules. For the new spin-resolved system the ground state ensemble, the ground state total energy, and the ground state spectral function of the impurity are determined in a many-particle treatment and in the Hartree-Fock approximation. In the following we discuss and compare our findings from both approaches. We restrict our discussion to the case of less-than-half filling of the localized orbitals, which reflects the situation in the rare earth's $4 f$-shell in the light rare-earth hexaborides.

\section{The ground state ensembles of the impurity in the many-particle treatment and in the Hartree-Fock approach}

For an integer ground state occupancy $\bar{N}_{f}^{\text {GS }}$ of the impurity, in the many-particle approach the only Slater determinants with non-zero weights in the ground state ensemble are those with $\bar{N}_{f}^{\mathrm{GS}}$ particles, where all particles have the same spin. Moreover, to satisfy the maximum entropy principle, all non-zero weights have the same value. Similar to the many-particle calculation, also in the HartreeFock approximation the Hund's coupling renders an occupancy of only one spin direction energetically favorable. Moreover, full occupancies of $\bar{N}_{f}^{\mathrm{GS}}$ orbitals in the same spin direction result in a lower total energy than other distributions of the impurity occupancy across the localized orbitals. Hence, in contrast to the many-particle calculation, in the Hartree-Fock approximation the ground state is a single Slater determinant in which all $\bar{N}_{f}^{\mathrm{GS}}$ particles are of the same spin. Both the spin and the orbital symmetries are broken.

For a fractional ground state occupancy $N_{f}^{\mathrm{GS}}$ of the impurity, only Slater determinants with either $\left\lfloor N_{f}^{\mathrm{GS}}\right\rfloor$ or $\left\lceil N_{f}^{\mathrm{GS}}\right\rceil$ particles, where all particles have the same spin, have non-zero weights in the ground state ensemble of the manyparticle approach. All Slater determinants with non-zero weight and with the same particle number have the same weight in the ground state ensemble to satisfy the maximum entropy principle. The weight ratio of all Slater determinants with $\left\lfloor N_{f}^{\mathrm{GS}}\right\rfloor$ particles to all Slater determinants with $\left\lceil N_{f}^{\mathrm{GS}}\right\rceil$ particles in the ground state ensemble is $\left(1-n_{f}^{\text {frac }}\right) / n_{f}^{\text {frac }}$. This reflects that the ground state total energy of the impurity for the ground state occupancy $N_{f}^{\mathrm{GS}}$ is a linear interpolation between the minimum total energies of the impurity for the adjacent integer occupancies $\left\lfloor N_{f}^{\mathrm{GS}}\right\rfloor$ and $\left\lceil N_{f}^{\mathrm{GS}}\right\rceil$. In the Hartree-Fock approximation the minimum total energy for the fractional ground state occupancy $N_{f}^{\mathrm{GS}}$ is obtained if $\left\lfloor N_{f}^{\mathrm{GS}}\right\rfloor$ localized orbitals are fully occupied and only one localized orbital has a fractional occupancy $n_{f}^{\text {frac }}$. All particles need to have the same spin. Hence, in the ground state ensemble of the impurity there are only 
5 The density of states of localized orbitals with fractional occupancy: study of a model system

two Slater determinants with non-zero weight: one Slater determinant with $\left\lfloor N_{f}^{\mathrm{GS}}\right\rfloor$ particles with weight $\left(1-n_{f}^{\text {frac }}\right)$ and one Slater determinant with $\left\lceil N_{f}^{\mathrm{GS}}\right\rceil$ particles with weight $n_{f}^{\text {frac }}$. In both Slater determinants the same $\left\lfloor N_{f}^{\mathrm{GS}}\right\rfloor$ spin orbitals are occupied. Again, the spin and orbital symmetries are broken.

\section{The ground state total energies of the impurity in the many-particle treatment and in the Hartree-Fock approach}

The ground state total energy takes the same value in the many-particle approach and in the Hartree-Fock approximation at an exchange scaling $\eta=1$. This is the case for an integer occupancy $\bar{N}_{f}^{\mathrm{GS}}$ and also for a fractional occupancy $N_{f}^{\mathrm{GS}}$. The reason for this is that Slater determinants are eigenstates not only of the effective non-interacting Hamiltonian $\hat{H}_{\text {loc }}^{\mathrm{HF}}$ of the Hartree-Fock approximation, eq. (5.135), but also of the many-particle Hamiltonian $\hat{H}_{\text {loc }}$, eq. (5.96). Each of the Slater determinants with $\bar{N}_{f}^{\mathrm{GS}}$ particles in one spin direction and no particle in the opposite spin direction has the same energy. Furthermore, in the case of a fractional occupancy it is important that the weight ratio of Slater determinants with $\left\lfloor N_{f}^{\mathrm{GS}}\right\rfloor$ particles to Slater determinants with $\left\lceil N_{f}^{\mathrm{GS}}\right\rceil$ particles is identical in the many-particle approach and in the Hartree-Fock approximation. The equal values of the ground state total energy from the Hartree-Fock approach and the many-particle treatment have an important implication. It means that, in the Hartree-Fock treatment, the ferromagnetic solution with a density matrix which is diagonal in the spin-orbital basis and which minimizes the total energy leads to a global minimum in the total energy. This is because the minimum of the total energy in the Hartree-Fock approximation can only be an upper boundary or equal to the true minimum of the total energy, but not lower in energy.

Let us next discuss the spectral functions that are obtained from the ground state ensembles of the many-particle calculation and the Hartree-Fock treatment of the model.

\section{The spectral function in the many-particle approach}

We can split the spectral function of the many-particle calculation into two parts. The first part contains all contributions that come from excitations in which a particle of the majority spin direction of a Slater determinant in the ground state ensemble is either inserted into or removed from the respective Slater determinant. Note that the Slater determinants of the ground state ensembles of the spinless model and the spin-dependent model are similar. "Stripping off" the orbitals of the (unoccupied) minority spin direction results in a Slater determinant as in the ground state ensemble of the spinless model. Moreover, the eigenvalue equation (5.100) with particles in only one spin direction is similar to eq. (5.14) of the spinless model. Hence, the first part of the spectral function has the same shape as the spectral function of the spinless model. However, the peak distance is rescaled to $U-J$. This part of the spectral function for a fractional occupancy of the impurity is depicted in the upper 
part of fig. 5.10. Further, for the spin-resolved model we have a second part of the spectral function, which contains all contributions coming from excitations where a particle of the minority spin direction of a Slater determinant in the ground state ensemble is inserted into the Slater determinant. Such a particle insertion leads to an occupancy of both spin directions, which is punished by Hund's coupling as it violates Hund's rules. Hence, the energies of excitations of this type are larger. In the case of an integer occupancy of the impurity all possible excitations of this type have the same energy $\varepsilon_{f}+U \bar{N}_{f}$ (all particles are of the same spin) so that there is one peak in this part of the spectral function. For a fractional occupancy of the impurity in the ground state ensemble all Slater determinants with spin occupancies $N_{\sigma}=\left\lfloor N_{f}\right\rfloor, N_{\bar{\sigma}}=0$, where $\sigma$ denotes the majority spin direction, contribute one excitation peak at energy $\varepsilon_{f}+U\left\lfloor N_{f}\right\rfloor$ and all Slater determinants with occupancies $N_{\sigma}=\left\lceil N_{f}\right\rceil, N_{\bar{\sigma}}=0$ contribute one excitation peak at energy $\varepsilon_{f}+U\left\lceil N_{f}\right\rceil$. Thus, this part of the spectral function has a total of two peaks. The peak separation is $U$. This part of the spectral function for a fractional occupancy of the impurity is depicted in the lower half of fig. 5.10.

\section{The spectral functions in the Hartree-Fock approach}

In the Hartree-Fock approach, we can compute excitation energies with the effective non-interacting Hamiltonian $\hat{H}_{\text {loc }}^{\mathrm{HF}}$ or with the interacting Hamiltonian $\hat{H}_{\text {loc }}$ and, thus, have two possible ways to determine a spectral function from the ground state ensemble. If the excitation energies are computed with the effective non-interacting Hamiltonian $\hat{H}_{\mathrm{loc}}^{\mathrm{HF}}$, we obtain a spectral function for the effective non-interacting system of the Hartree-Fock approach. In this case the excitation energies correspond to the single-particle energies $\{\xi\}$ given by eq. (5.136). Importantly, an energy level $\xi_{m \sigma}$ of an orbital $m \sigma$ depends on the average occupancy $\left\langle\hat{n}_{m \sigma}\right\rangle_{\mathrm{HF}}$ of the orbital $m \sigma$ within the ensemble of the impurity. We split the spectral function into two parts.

The first part contains the contributions from excitations in which particles of the majority spin direction are either inserted into or removed from Slater determinants of the ground state ensemble. Note that the ground state ensembles of the impurity for the spin-resolved model and for the spinless model are similar. Moreover, at $\eta=1$ for an orbital of the majority spin direction, the energy $\xi_{m \sigma}$, eq. (5.136), is similar to that of the spinless model, eq. (5.76). Hence, the spectral function of the majority spin direction has the same shape as the spectral function of the spinless model. However, the energy that determines the distance between the excitation peaks is rescaled to $\eta(U-J)$. For an integer occupancy of the impurity, the single-particle energies $\{\xi\}$ can take two distinct values in the majority spin direction, depending on whether the occupancy of the corresponding localized orbital is 0 or 1 . These are $\varepsilon_{f}+(U-\eta J) N_{f}$ and $\varepsilon_{f}+(U-\eta J) N_{f}-\eta(U-J)$, respectively. The spectral function of the majority spin direction has a two-peak shape, where the occupied and unoccupied levels are an energy $\eta(U-J)$ apart. For $\eta=1$ we obtain the same average spectral function over the localized orbitals as in the many-body calculation. In the case of a fractional occupancy of the impurity, the spectral function of the ma- 
jority spin direction has a three-peak shape. One fractionally occupied orbital is found at the Fermi level in between the energy levels of the fully occupied orbitals at energy $E_{\mathrm{F}}-\eta(U-J)\left(1-n_{f}^{\text {frac }}\right)$ and the empty orbitals at energy $E_{\mathrm{F}}+\eta(U-J) n_{f}^{\text {frac }}$. The fully occupied orbitals and the empty orbitals are an energy $\eta(U-J)$ apart. For a fractional occupancy of the impurity, the first part of the spectral function is depicted in the upper half of fig. 5.11. Evidently, the spectral function of the effective non-interacting system of the Hartree-Fock approach differs from the spectral function of the interacting impurity from the many-body approach because different excitation energies are obtained with the Hamiltonians (5.96) and (5.135), respectively.

The second part of the spectral function contains all contributions from excitations, where a particle of the minority spin direction is inserted into the Slater determinants of the ground state ensemble. In the effective non-interacting system, such an excitation has the same energy for all Slater determinants of the ground state ensemble because the energies (5.136) depend on the average occupancy of the impurity and not on the particle number within the individual Slater determinants. Thus, for occupancies $N_{\sigma}=N_{f}, N_{\bar{\sigma}}=0$, where $\sigma$ is the majority spin direction, the spectral function of the minority spin direction has a single feature at energy $\varepsilon_{f}+U N_{f}$. This energy is higher by $\eta J N_{f}$ than the energy of the unoccupied states of the majority spin direction, reflecting that an occupancy of both spin directions is unfavorable due to the Hund's rule coupling. In the case of an integer occupancy and at $\eta=1$, the average spectral function of the effective non-interacting system over all localized orbitals is equal to that of the interacting impurity. For a fractional occupancy of the impurity, the orbitals in the minority spin direction are all found at the same energy of $E_{\mathrm{F}}+\eta J N_{f}+\eta(U-J) n_{f}^{\text {frac }}$. The second part of the spectral function is depicted in the lower half of fig. 5.11 for a fractional occupancy of the impurity. At $\eta=1$ it differs from that of the many-body calculation, which, by contrast, has one feature coming from particle-insertion excitations of the $\left\lfloor N_{f}\right\rfloor$-particle Slater determinants of the ground state ensemble and one peak coming from particle-insertion excitations of the $\left\lceil N_{f}\right\rceil$-particle Slater determinants.

In the other possibility to obtain a spectral function from the impurity ensemble of the Hartree-Fock approximation we compute the excitation energies with the interacting Hamiltonian $\hat{H}_{\text {loc }}$. These excitation energies are identical to the excitation energies computed in the many-particle approach. However, in contrast to the many-particle treatment of the model, we do not obtain the same spectral function for each spin orbital because the spin and orbital symmetries are broken in the ground state ensemble in the Hartree-Fock approach. Due to the broken spin symmetry, the spectral functions of spin orbitals of the majority spin direction differ from the spectral functions of spin orbitals of the minority spin direction. Moreover, in the majority spin direction also the orbital symmetry is broken and we obtain different spectral functions for an occupied orbital, an unoccupied orbital, and the fractionally occupied orbital. In all Slater determinants of the ground state ensemble, all orbitals are unoccupied in the minority spin direction and the insertion of a particle of the minority spin into any orbital is associated with the same excitation energy. Hence, all spin orbitals of the minority spin direction have the same spectral function. While 
the spectral functions of the individual spin orbitals differ from their analogs of the many-particle treatment, this is not the case for the average spectral function over all spin orbitals, which is the same as in the many-particle treatment of the model.

It is evident that the spectral function of the particles of the majority spin direction in the spin-resolved model is the same as in the spinless model system, except for the altered interaction strength. The advantage of the spin-resolved model is that we also obtain a spectral function for the minority spin direction. This and the inclusion of a Hund's coupling exchange allow for a more rigorous comparison with the DOSs of our DFT simulations. In the next section we compare the spectral functions of the spin-resolved model system and the $4 f$ projected DFT DOSs of the light rare-earth hexaborides. Moreover, we use our results from the analysis of the spin-resolved model system to provide a detailed rationale for the positions of the features in the $4 f$-projected DFT DOSs of the light rare-earth hexaborides.

\subsection{Comparison of the spectral function of the model to the DFT DOSs of the light rare-earth hexaborides}

This section is divided into two parts. First, we compare the spectral function of the spin-resolved model system to the DFT DOSs of $\mathrm{CeB}_{6}, \mathrm{PrB}_{6}$, and $\mathrm{NdB}_{6}$. In the the second part, we alter the existing model so that it more accurately reflects the situation in the DFT simulations with the PBE0r hybrid functional. In particular, the altered model is constructed from an LSDA-like contribution, the exchange term of the spin-resolved model, and a model term for the double counting in the PBE0r hybrid functional. It is shown that the altered model is well suited to describe the $4 f$-projected DOSs from our DFT bulk simulations of the light rare-earth hexaborides.

\subsubsection{Spectral function of the spinless model versus the DFT DOSs of the light rare-earth hexaborides}

For an integer occupancy of the impurity, the impurity spectral function of the effective non-interacting system of the Hartree-Fock approach matches the spectral function of the many-body treatment. It shows two distinct features: one feature coming from the occupied orbitals and one feature from the unoccupied orbitals of the effective non-interacting system. The respective energies correspond to the energies of particle-insertion and particle-removal excitations of the ground state Slater determinants of the interacting system.

Our DFT simulations of $\mathrm{CeB}_{6}$ result in an integer occupancy of the $4 f$ shell in the ground state. The $4 f$-projected DOSs of these simulations have a twopeak shape that is similar to the impurity spectral function of the model system. We can bring the DFT DOS into agreement with experimental photoemission spectra by tuning the exchange mixing factor of the PBEOr hybrid functional 
5 The density of states of localized orbitals with fractional occupancy: study of a model system

to a value of 0.15 . In this case the DOS of the effective non-interacting KohnSham system matches the excitation spectrum of the real interacting material. This is analogous to the situation in the model system.

Similarly, we find a two-peak shape also for the $4 f$-projected DOSs of $\operatorname{PrB}_{6}$ that are of TYPE II. Also in this case the DFT DOS can be brought into agreement with photoemission spectra by tuning the exchange mixing factor of the PBE0r hybrid functional to a value of 0.225 . We remark that the integration of the $4 f$-projected DOS gives a total occupancy of 2.25 for the $4 f$ shell of $\operatorname{PrB}_{6}$ at an exchange mixing factor of 0.225 , which is larger than an integer number of 2. Note, however, that an overestimation in the occupancy of the $4 f$ states can occur for technical reasons. These include an imprecision due to the projection of the DOS onto the $4 f$ states and also the broadening of the DOS so that the contributions of $4 f$ states closely above the Fermi level leak below the Fermi level. The $4 f$-projected DOS is in accordance with the spectral function of the model at an integer occupancy of the impurity and also with the experimental photoemission spectra. Hence, we deem the underlying many-particle state to have an integer occupancy of the $4 f$ orbitals.

If the total energy of the model is minimized for a fractional occupancy of the impurity, the spectral function of the localized states in the effective non-interacting system of the Hartree-Fock approach has a three-peak shape in the majority spin direction and exhibits a single, fractionally occupied orbital at the Fermi level. Such a spectrum strongly resembles the $4 f$-projected DOSs of our simulations of $\mathrm{PrB}_{6}$ (for TyPE III DOSs) and $\mathrm{NdB}_{6}$ with the PBE0r hybrid functional. These $4 f$-projected DOSs also have a three-peak shape with a single, fractionally occupied state in the majority spin direction at the Fermi level. Moreover, for $\mathrm{PrB}_{6}$ at an exchange mixing of 0.15 the fractionally occupied state has an occupancy of $\sim 0.3$ and is located close to the unoccupied $4 f$ states. In contrast, for $\mathrm{NdB}_{6}$ the fractional occupancy is $\sim 0.6$ and the fractionally occupied state is close to the occupied $4 f$ states. This is in accordance with the spectral function of the effective non-interacting system of the Hartree-Fock treatment of the model, where the fractionally occupied state is closer in energy to the fully occupied states if the fractional occupancy is large, and closer to the completely unoccupied states if the fractional occupancy is small. Furthermore, we find this behavior of the DOS also in fig. 4.13, where the simulation of $\mathrm{NdB}_{6}$ with a set of $(2,1,1,1)$ projector functions for the $\mathrm{Nd}(s$, $p, d, f)$ orbitals results in a smaller fractional occupancy than the simulation with $(2,2,1,1)$ projector functions.

In the preceding paragraphs we compared the $4 f$-projected DOSs from the DFT simulations of the rare-earth hexaborides with the spectral function of the localized orbitals of the effective non-interacting system of the Hartree-Fock approximation to the model. We found concordant behavior in the following points.

1. In both systems a two-peak spectrum for the localized orbitals of the majority spin direction occurs for an integer ground state occupancy of the localized orbitals.

2. In both systems a three-peak spectrum for the localized orbitals of the 
majority spin direction with one fractionally occupied orbital at the Fermi level occurs for a fractional ground state occupancy of the localized orbitals.

3. The spectra for the localized orbitals of both systems show concordant behavior with respect to changes in the exchange mixing strength.

4. The spectra for the localized orbitals of both systems show the same behavior with respect to changes of the fractional occupancy of the localized orbitals.

This indicates that our model system is well suited to describe the effect of the exact exchange term within the PBE0r hybrid functional onto the DFT data of the rare-earth hexaborides. Moreover, we can now rationalize the mismatch between the DFT DOSs and the experimental photoemission spectra for $\mathrm{NdB}_{6}$. This is discussed in the following.

For localized local orbitals like the $4 f$ states of the lanthanide ions, due to the large interaction in the $4 f$ shell, the effect of the exchange mixing in the PBE0r hybrid functional is strong. Moreover, the hybridization of the $4 f$ states with metallic states within the rare-earth hexaborides is small, so that we have a situation that corresponds to that in our model system. This explains the correspondence between the shapes of the $4 f$-projected DOSs of the rare-earth hexaborides in our DFT simulations and the spectral function of the effective non-interacting system of the Hartree-Fock approximation to our model. Moreover, we know that the spectral function of the effective noninteracting system does not represent the spectral function of the interacting system in the case of a fractional occupancy of the localized orbitals. We have thus found a rationale for the mismatch between the DOSs of our DFT simulations of $\mathrm{PrB}_{6}$ and $\mathrm{NdB}_{6}$, where fractional ground state occupancies of the $4 f$ shell have been obtained, and experimental photoemission spectra.

Based on the analogy to the model system, we can expect that this problem will appear in general for metallic materials with a small hybridization coupling between a metallic band and strongly localized orbitals, if the ground state occupancy of the localized orbitals is fractional. One approach to this problem could be to extend the DFT algorithm such that multiple Slater determinants of the Kohn-Sham states are tracked during a simulation. In such an algorithm each Kohn-Sham state would receive multiple associated Kohn-Sham energies - one for each Slater determinant of the ground state ensemble. If the ground state ensemble of the Kohn-Sham system was known, it would be possible to compute the ground state DOS as the average of the DOSs coming from the individual Slater determinants of the ground state ensemble. The computation of the DOS in the suggested approach is similar to the computation of the spectral function from the ground state ensemble of the Hartree-Fock approach with the original interacting Hamiltonian. Hence, the resulting ground state DOS is expected to give a better description of the excitation spectrum of the real material. However, details on the computational scheme of such an extended DFT algorithm and an efficient implementation require further work. 
5 The density of states of localized orbitals with fractional occupancy: study of a model system

We remark that, according to the model study, the possibility to find a fractional occupancy of the localized orbitals should increase for smaller mixing factors of the Hartree-Fock exchange in the PBE0r hybrid functional. The solution of the model with zero self-interaction correction, $\eta=0$, allows an integer occupancy only for singular values of $\varepsilon_{f}$, while in the case of a full self-interaction compensation with $\eta=1$ broad plateaus of integer ground state occupancy occur in the graph of $N_{f}^{\mathrm{GS}}$, fig. 5.8. A mixing factor $0<\eta<1$ leads to smaller plateaus and interpolates between both of the extreme cases. Hence, for simulations of materials with the PBE functional, which corresponds to $\eta=$ 0 , a fractional occupancy of the localized orbitals should occur more frequently than in simulations with the PBE0r hybrid functional. This also suggests that choosing a too small exchange mixing factor can lead to a fractional occupancy of localized orbitals whereas for the correct mixing factor an integer occupancy would be obtained.

Our model calculation demonstrates in detail how the shapes of the $4 f$ projected DFT DOSs of the rare-earth hexaborides originate from the exact exchange term of the PBE0r hybrid functional. The contribution of the exact exchange term to the one-particle matrix in the Hartree-Fock approximation reads

$$
\frac{\partial E_{\mathrm{x}}^{\mathrm{HF}}}{\partial\left\langle\hat{f}_{a}^{\dagger} \hat{f}_{b}\right\rangle_{\mathrm{HF}}}=-\sum_{c d} U_{d a b c}\left\langle\hat{f}_{d}^{\dagger} \hat{f}_{c}\right\rangle_{\mathrm{HF}}=-\sum_{c d} U_{d a b c} \rho_{c d} .
$$

In DFT simulations with the PBE0r hybrid functionial, it enters the exchange contribution to a Kohn-Sham energy level, eq. (5.2), for each site of local orbitals. For a Kohn-Sham state that resembles a localized local orbital, the exchange contribution to the Kohn-Sham energy is analogous to the exchange contribution to the energy of a localized state in our model system,

$$
\Delta \xi_{m \sigma}^{\mathrm{x}}=\frac{\partial E_{\mathrm{loc}, \mathrm{x}}^{\mathrm{HF}}}{\partial\left\langle\hat{n}_{m \sigma}\right\rangle_{\mathrm{HF}}}=-J N_{\sigma}-(U-J)\left\langle\hat{n}_{m \sigma}\right\rangle_{\mathrm{HF}} .
$$

The exchange introduces a dependence of the energy level on the occupancy $\left\langle\hat{n}_{m \sigma}\right\rangle_{\mathrm{HF}}$. Due to the exact exchange term the DFT DOSs show a better compensation of the self-interaction error than with the conventional PBE functional.

The complete exchange admixture in the PBE0r hybrid functional also includes a double counting term that is neglected in the present model. In the next section we combine the exact exchange term and a model for the double counting part for a more rigorous comparison with the DFT data. 


\subsubsection{Including a model for the double counting}

In this section we rationalize the positions of the peaks within the $4 f$-projected DOSs of our DFT simulations of $\mathrm{CeB}_{6}, \mathrm{PrB}_{6}$, and $\mathrm{NdB}_{6}$ with the PBE0r hybrid functional. For this purpose let us assume that the total energy of an impurity of $4 f$ orbitals has the form

$$
E_{\mathrm{loc}}=E_{\mathrm{loc}}^{\mathrm{LSDA}}+\eta \Delta E_{\mathrm{loc}, \mathrm{x}} .
$$

The first part on the right hand side of the equation, $E_{\text {loc }}^{\mathrm{LSDA}}$, is the total energy of the impurity as obtained in the local spin density approximation. Further, the second term on the right hand side of the equation denotes an admixture of exact exchange, where

$$
\Delta E_{\mathrm{loc}, \mathrm{x}}=E_{\mathrm{loc}, \mathrm{x}}^{\mathrm{HF}}-E_{\mathrm{loc}, \mathrm{x}}^{\mathrm{DC}} .
$$

Equation (5.161) can be understood as an analogy to the correction to the PBE exchange-correlation functional that is introduced through the exchange mixing within the PBE0r hybrid functional. From our model calculation, we know that the exact exchange energy of the impurity reads

$$
E_{\mathrm{loc}, \mathrm{x}}^{\mathrm{HF}}=-\frac{J}{2} \sum_{\sigma} N_{\sigma}^{2}-\frac{U-J}{2} \sum_{m \sigma} n_{m \sigma}^{2}
$$

For simplicity of notation, here and in the remainder of this chapter, we denote the occupancy of a spin orbital $m \sigma$ as $n_{m \sigma}$.

Let us also give an estimate for the double counting correction $E_{\mathrm{loc}, \mathrm{x}}^{\mathrm{DC}}$. For this purpose, we can rely on a form of the double counting correction that is used in the LSDA $+U$ technique. In LSDA $+U$, the double counting includes the Hartree interaction of the local orbitals which we can strip off since our double counting focuses on the exchange term only. We follow the idea that the LSDA is similar to a mean-field approximation [95]. Because the mean-field approximation to a many-fermion system is just the Hartree-Fock approximation, we can assume that the double counting correction takes a form similar to eq. (5.162). However, the treatment of the self-interaction is incomplete within the LSDA and the LSDA lacks a precise treatment of the individual localized orbitals. Hence, the last term of eq. (5.162), which is orbital-dependent, is approximated in terms of an expression that depends on the total spin occupancy $N_{\sigma}$. In the context of the LSDA $+U$ technique two different versions of the double counting are well established [89, 95, 96, 151-153]. First, we have the around mean field (AMF) approximation,

$$
n_{m \sigma} \approx \frac{N_{\sigma}}{N_{\text {orb }}},
$$

where all localized orbitals receive the same average occupancy per spin direction. This results in

$$
E_{\mathrm{loc}, \mathrm{x}}^{\mathrm{DC}, \mathrm{AMF}} \approx-\frac{1}{2}\left[J^{\mathrm{LSDA}}+\frac{U^{\mathrm{LSDA}}-J^{\mathrm{LSDA}}}{N_{\mathrm{orb}}}\right] \sum_{\sigma} N_{\sigma}^{2} .
$$


5 The density of states of localized orbitals with fractional occupancy: study of a model system

Note that, in principle, for our double counting we construct a second model for the purpose of mimicking the LSDA to our impurity. We cannot expect the interaction parameters $U^{\mathrm{LSDA}}$ and $J^{\mathrm{LSDA}}$ to be identical to the parameters $U$ and $J$ of the Hartree-Fock exchange because they are obtained in different ways.

The second form of the double counting is the fully localized limit (FLL), which corresponds to the approximation

$$
\sum_{m} n_{m \sigma}^{2} \approx N_{\sigma}
$$

For the FLL, the double counting energy reads

$$
E_{\mathrm{loc}, \mathrm{x}}^{\mathrm{DC}, \mathrm{LL}} \approx-\frac{J^{\mathrm{LSDA}}}{2} \sum_{\sigma} N_{\sigma}^{2}-\frac{U^{\mathrm{LSDA}}-J^{\mathrm{LSDA}}}{2} \sum_{\sigma} N_{\sigma} .
$$

The FLL gives the correct mean exchange energy if all orbital occupancies are either 0 or 1 .

Petukhov et al. argued that the occupancies of the localized orbitals in real materials are in between the uniform occupancy of the AMF and the 0-or-1 occupancy of the FLL [152]. Hence, it was suggested to take a linear interpolation of the AMF and FLL terms for a more accurate double counting [152, 153]. We follow the idea to take the double counting as a linear interpolation of the form $E_{\mathrm{loc}, \mathrm{x}}^{\mathrm{DC}}=(1-\alpha) E_{\mathrm{loc}, \mathrm{x}}^{\mathrm{DC}, \mathrm{AMF}}+\alpha E_{\mathrm{loc}, \mathrm{x}}^{\mathrm{DC}, \mathrm{FL}}$ as this allows to easily consider both versions of the LSDA $+U$ double counting. The AMF double counting is recovered for $\alpha=0$ and the FLL double counting is obtained for $\alpha=1$.

According to the preceding paragraphs, our double counting energy has the form

$$
\begin{aligned}
E_{\mathrm{loc}, \mathrm{x}}^{\mathrm{DC}} & \approx-\frac{J^{\mathrm{LSDA}}}{2} \sum_{\sigma} N_{\sigma}^{2}-\frac{U^{\mathrm{LSDA}}-J^{\mathrm{LSDA}}}{2} \sum_{\sigma} N_{\sigma}\left[\frac{N_{\sigma}}{N_{\mathrm{orb}}}(1-\alpha)+\alpha\right] \\
& =-\frac{J_{\mathrm{eff}}^{\mathrm{LSDA}}(\alpha)}{2} \sum_{\sigma} N_{\sigma}^{2}-\alpha \frac{U^{\mathrm{LSDA}}-J^{\mathrm{LSDA}}}{2} \sum_{\sigma} N_{\sigma}
\end{aligned}
$$

For a concise notation, we introduce the symbol

$$
J_{\mathrm{eff}}^{\mathrm{LSDA}}(\alpha):=J^{\mathrm{LSDA}}+(1-\alpha) \frac{U^{\mathrm{LSDA}}-J^{\mathrm{LSDA}}}{N_{\text {orb }}} .
$$

With eqs. (5.162) and (5.168) the exchange term (5.161) becomes

$$
\begin{aligned}
\Delta E_{\mathrm{loc}, \mathrm{x}}(\alpha) \approx & -\frac{\left(J-J_{\mathrm{eff}}^{\mathrm{LSDA}}(\alpha)\right)}{2} \sum_{\sigma} N_{\sigma}^{2}+\frac{\alpha\left(U^{\mathrm{LSDA}}-J^{\mathrm{LSDA}}\right)}{2} \sum_{\sigma} N_{\sigma} \\
& -\frac{(U-J)}{2} \sum_{m \sigma} n_{m \sigma}^{2} .
\end{aligned}
$$


5.9 Comparison of the spectral function of the model to the DFT DOSs of the light rare-earth hexaborides

The contribution to the energy level within an effective system of non-interacting particles is obtained via the derivative

$$
\frac{\partial \Delta E_{\mathrm{loc}, \mathrm{x}}}{\partial n_{m \sigma}}=-\left(J-J_{\mathrm{eff}}^{\mathrm{LSDA}}(\alpha)\right) N_{\sigma}+\frac{\alpha\left(U^{\mathrm{LSDA}}-J^{\mathrm{LSDA}}\right)}{2}-(U-J) n_{m \sigma} .
$$

Hence, based on the above estimate of the exchange mixing term, the singleparticle energy levels are of the form

$$
\xi_{m \sigma}^{\mathrm{PBE} 0 \mathrm{r}}=\xi_{\sigma}^{\mathrm{LSDA}}-\eta\left(J-J_{\mathrm{eff}}^{\mathrm{LSDA}}\right) N_{\sigma}+\frac{\eta \alpha\left(U^{\mathrm{LSDA}}-J^{\mathrm{LSDA}}\right)}{2}-\eta(U-J) n_{m \sigma} .
$$

The first term on the right hand side of the equation denotes the contribution from the LSDA. The LSDA includes an individual treatment of both spin directions but lacks a careful treatment of individual localized orbitals. Hence, $\xi_{\sigma}^{\mathrm{LSDA}}$ is assumed to depend on the spin only. The other terms on the right hand side of eq. (5.172) are the correction due to the exchange mixing.

Based on eq. (5.172), we find the one-particle energy levels relative to the Fermi level $E_{\mathrm{F}}$ that are given in the following. We assume that $\sigma=\uparrow$ is the majority spin direction and the spin occupancies are $N_{\uparrow}=N_{f}$ and $N_{\downarrow}=0$. For an integer occupancy of the impurity, the energy levels of the occupied orbitals in the spin- $\uparrow$ direction, the unoccupied orbitals in the spin- $\uparrow$ direction, and the unoccupied orbitals in the spin- $\downarrow$ direction are

$$
\begin{gathered}
\xi_{\mathrm{occ}, \uparrow}^{\mathrm{PBE} 0 \mathrm{r}}-E_{\mathrm{F}}=\xi_{\uparrow}^{\mathrm{LSDA}}-E_{\mathrm{F}}-\eta\left(J-J_{\mathrm{eff}}^{\mathrm{LSDA}}\right) N_{f} \\
-\eta\left[(U-J)-\frac{\alpha\left(U^{\mathrm{LSDA}}-J^{\mathrm{LSDA}}\right)}{2}\right], \quad \\
\xi_{\mathrm{unocc}, \uparrow}^{\mathrm{PBE} 0 \mathrm{r}}-E_{\mathrm{F}}=\xi_{\uparrow}^{\mathrm{LSDA}}-E_{\mathrm{F}}-\eta\left(J-J_{\mathrm{eff}}^{\mathrm{LSDA}}\right) N_{f}+\frac{\eta \alpha\left(U^{\mathrm{LSDA}}-J^{\mathrm{LSDA}}\right)}{2},
\end{gathered}
$$

and

$$
\xi_{\text {unocc }, \downarrow}^{\mathrm{PBE} 0 \mathrm{r}}-E_{\mathrm{F}}=\xi_{\downarrow}^{\mathrm{LSDA}}-E_{\mathrm{F}}+\frac{\eta \alpha\left(U^{\mathrm{LSDA}}-J^{\mathrm{LSDA}}\right)}{2},
$$

respectively. Moreover, for a fractional occupancy of the impurity we find the energy levels

$$
\begin{array}{r}
\xi_{\mathrm{occ}, \uparrow}^{\mathrm{PBE} 0 \mathrm{r}}-E_{\mathrm{F}}=\xi_{\mathrm{occ}, \uparrow}^{\mathrm{PBE} 0 \mathrm{r}}-\xi_{\text {frac }, \uparrow}^{\mathrm{PBE} 0 \mathrm{r}}=-\left(1-n_{f}^{\mathrm{frac}}\right) \eta(U-J), \\
\xi_{\mathrm{unocc}, \uparrow}^{\mathrm{PBE} 0 \mathrm{r}}-E_{\mathrm{F}}=\xi_{\mathrm{unocc}, \uparrow}^{\mathrm{PBE} 0 \mathrm{r}}-\xi_{\text {frac }, \uparrow}^{\mathrm{PBE} 0 \mathrm{r}}=n_{f}^{\mathrm{frac}} \eta(U-J),
\end{array}
$$


5 The density of states of localized orbitals with fractional occupancy: study of a model system

and

$$
\begin{aligned}
\xi_{\text {unocc }, \downarrow}^{\mathrm{PBE} 0 \mathrm{r}}-E_{\mathrm{F}} & =\xi_{\text {unocc, } \downarrow}^{\mathrm{PBE} 0 \mathrm{r}}-\xi_{\mathrm{frac}, \uparrow}^{\mathrm{PBE} \uparrow \mathrm{r}} \\
& =\xi_{\downarrow}^{\mathrm{LSDA}}-\xi_{\uparrow}^{\mathrm{LSDA}}+\eta\left(J-J_{\mathrm{eff}}^{\mathrm{LSDA}}\right) N_{f}+\eta(U-J) n_{f}^{\mathrm{frac}} .
\end{aligned}
$$

Note that, for a fractional occupancy of the impurity, the fractionally occupied orbital is situated at the Fermi level. Hence, to compute the position of an energy level relative to the Fermi level, we subtract the energy $\xi_{\text {frac }, \uparrow}^{\mathrm{PBEOr}}$ of the fractionally occupied state.

In our model we work with screened interaction parameters $U$ and $J$, which can be scaled by the exchange mixing factor $\eta$. This is in contrast to the situation in DFT, where the exchange term is obtained from the bare interaction and scaled with a different exchange mixing factor $\eta^{\text {DFT }}$. To link the model calculation with the DFT simulations we define $\eta_{0}^{\text {DFT }}$ as the "optimum" value of the exchange mixing factor within the PBE0r hybrid functional at which the self-interaction is exactly compensated. Evidently, this definition only works under the idealistic assumption that the DFT results are exact for an exchange mixing factor $\eta_{0}^{\mathrm{DFT}}$. Then, the exchange mixing factor of the PBE0r hybrid functional $\eta^{\mathrm{DFT}}$ is related to the model parameter $\eta$ via the "optimum" value $\eta_{0}^{\mathrm{DFT}}$ for the exchange mixing in DFT,

$$
\eta=\frac{\eta^{\text {DFT }}}{\eta_{0}^{\text {DFT }}}
$$

The bare (unscreened) Coulomb parameter $U_{\text {bare }}$ and the bare Hund parameter $J_{\text {bare }}$ in the rare-earth $4 f$ shell are related to the screened Coulomb and Hund parameters $U$ and $J$ of the model system via

$$
U_{\text {bare }}=\frac{U}{\eta_{0}^{\mathrm{DFT}}} \quad \text { and } \quad J_{\text {bare }}=\frac{J}{\eta_{0}^{\mathrm{DFT}}} .
$$

Hence, $\eta_{0}^{\text {DFT }}$ is a constant which mediates between the screened and bare interactions. This conversion has to be kept in mind when comparing the model results with the results from our DFT simulations in the further course of this section.

Let us now determine estimates for the effective bare interaction strengths $U_{\text {bare }}-J_{\text {bare }}$ within the light rare-earth hexaborides $\mathrm{CeB}_{6}, \mathrm{PrB}_{6}$, and $\mathrm{NdB}_{6}$. For this purpose we use the distances between the occupied and unoccupied peaks of the majority spin direction as obtained from the $4 f$-projected DOSs of our DFT simulations, see fig. 4.15. From eq. (5.172) we obtain for the distance between the unoccupied and occupied states of the majority spin direction

$$
\xi_{\text {unocc }, \uparrow}^{\mathrm{PBE} 0 \mathrm{r}}-\xi_{\mathrm{occ}, \uparrow}^{\mathrm{PBE},}=\eta(U-J)=\eta^{\mathrm{DFT}}\left(U_{\text {bare }}-J_{\text {bare }}\right) .
$$

From eq. (5.182) it is clear that the slope of a fit to the data in fig. 4.15 is a measure for an effective interaction $U_{\text {bare }}-J_{\text {bare }}$ in the rare-earth $4 f$ shell. We obtain effective bare interaction strengths of $(24.2 \pm 0.3) \mathrm{eV}$ for $\mathrm{CeB}_{6}$, 
$(26.0 \pm 0.2) \mathrm{eV}$ for $\mathrm{PrB}_{6}$, and $(28.0 \pm 0.1) \mathrm{eV}$ for $\mathrm{NdB}_{6}$. As expected the interaction strength increases when moving from $\mathrm{CeB}_{6}$ to $\mathrm{NdB}_{6}$. We remark that eq. (5.182) is analogous to eq. (5.141) because the double counting does not care about the individual occupancies of the localized orbitals.

In the next step we relate the equations (5.173) - (5.179) for the peak positions as determined in the model calculation to the peak positions in the DOSs of our DFT simulations of $\mathrm{CeB}_{6}, \mathrm{PrB}_{6}$, and $\mathrm{NdB}_{6}$. For a visual representation we plot the peak positions from the DFT simulations for different values of the exchange mixing factor in fig. 5.12. Moreover, in the plot we include the evolution of the orbital energies with respect to changes in the exchange mixing factor $\eta$ according to equations (5.173) - (5.179). The parameters for the curves (5.173) - (5.179) are determined from fits to the DFT data as described in the following. First, the effective interaction strength $U-J$ is just the slope of the fits in fig. 4.15 as discussed above. Second, the difference $\xi_{\downarrow}^{\mathrm{LSDA}}-\xi_{\uparrow}^{\mathrm{LSDA}}$ is obtained from the DFT data as the mean value of the energy difference between the unoccupied states of both spin directions across the simulations with different exchange mixing factors. Third, for integer $4 f$ occupancies, the slope $\alpha\left(U^{\mathrm{LSDA}}-J^{\mathrm{LSDA}}\right) / 2$ and the value $\xi_{\uparrow}^{\mathrm{LSDA}}$ are obtained from a linear fit to the positions of the unoccupied states of the spin- $\uparrow$ direction.

The distances between the unoccupied energy levels of the two spin directions remain constant within the margin of error in the DFT data of all three hexaborides. According to our model this indicates that $J=J_{\text {eff }}^{\mathrm{LSDA}}$. Hence, the double counting energy appears to compensate the first term of the exchange energy (5.162).

For $\mathrm{CeB}_{6}$ and $\mathrm{PrB}_{6}$, with the exception of $\operatorname{PrB}_{6}$ at an exchange mixing of 0.15 , we obtain $4 f$-projected DOSs with two peaks in the majority spin direction from the ground states of the DFT simulations. The Fermi level lies in between the occupied and unoccupied $4 f$ levels. This matches the situation for an integer occupancy of the impurity in our model system. From fig. 5.12 it is clear that the positions of the $4 f$ levels from our DFT data of $\mathrm{CeB}_{6}$ and $\mathrm{PrB}_{6}$ can indeed be described by the functions (5.173) - (5.175) of our model calculation for an integer occupancy of the localized orbitals. The compensation of the self-interaction due to the exchange mixing only affects occupied orbitals and lowers their energy by $\eta(U-J)$. Further, note that the term $\eta \alpha\left(U^{\mathrm{LSDA}}-J^{\mathrm{LSDA}}\right) / 2$, which comes from the FLL double counting correction, is particularly important for the curves to match the DFT data. In the limit of the AMF double counting correction (where $\alpha=0$ ), the latter term is absent and the model would predict the unoccupied levels to remain at a constant energy, even when changing the exchange mixing strength. This is clearly not the case for the $4 f$ levels from the DFT simulations. Moreover, the energy of occupied orbitals would follow a steeper descent for an increasing exchange mixing strength. On the other hand, it appears that the DFT data can be correctly described with a double counting in the FLL limit (where $\alpha=1$ ). We note, however, that the slopes of the graphs of the unoccupied levels, which, for $\alpha=1$, are a measure for half the effective interaction strength between the localized orbitals, are $(5.9 \pm 0.3) \mathrm{eV}$ for $\mathrm{CeB}_{6}$ and $(2.5 \pm 0.4) \mathrm{eV}$ for $\mathrm{PrB}_{6}$. This is much smaller than half the bare effective interaction strengths 
5 The density of states of localized orbitals with fractional occupancy: study of a model system

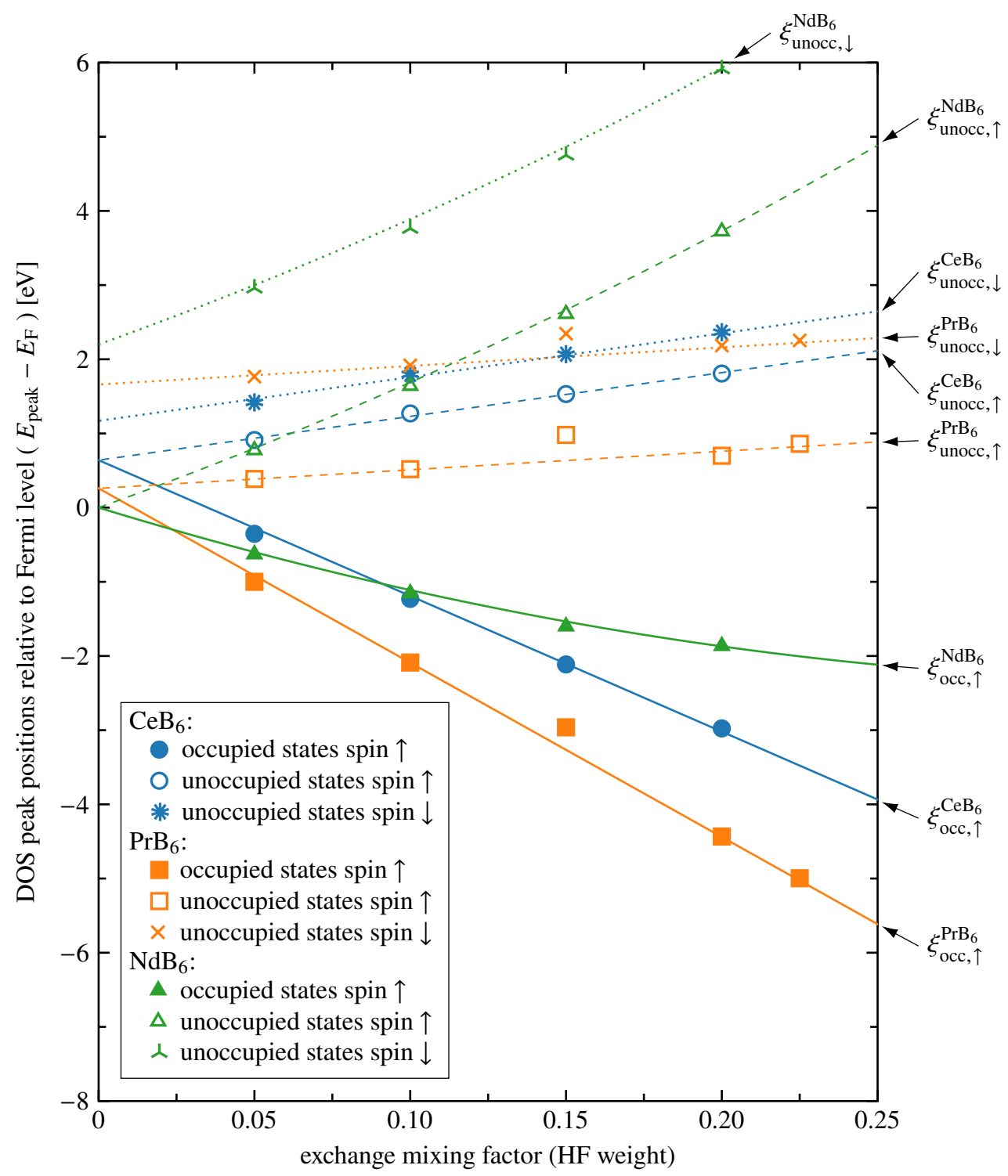

Figure 5.12: Dependence of the $4 f$ energy levels of $\mathrm{CeB}_{6}, \mathrm{PrB}_{6}$, and $\mathrm{NdB}_{6}$ relative to the Fermi level on the exchange mixing factor. Datapoints: energies of the fully occupied and empty $4 f$ states relative to the Fermi level, obtained from our DFT simulations of $\mathrm{CeB}_{6}$, $\mathrm{PrB}_{6}$, and $\mathrm{NdB}_{6}$. (Same data as in fig. 4.14.) Lines: estimated evolution of the $4 f$ energies against the exchange mixing factor according to our model. More information is given in the text. 
of $(12.1 \pm 0.2) \mathrm{eV}$ for $\mathrm{CeB}_{6}$ and $(13.0 \pm 0.1) \mathrm{eV}$ for $\operatorname{PrB}_{6}$, respectively. In the case of a double counting that is a linear interpolation between the AMF and the FLL, the parameter $\alpha$ scales the interaction parameters $U^{\mathrm{LSDA}}$ and $J^{\mathrm{LSDA}}$ from the LSDA, which in principle allows for a better agreement with the interaction parameters $U$ and $J$ of the model atom.

A double counting term that raises the energies of the localized orbitals like in the FLL approximation could also serve as a rationale for the increase in the energy of the unoccupied $4 f$ levels in $\mathrm{LaB}_{6}$ that was described in section 4.3.

In comparison to $\mathrm{CeB}_{6}$ and $\mathrm{PrB}_{6}$, the energies of the $4 f$ states in our DFT data on $\mathrm{NdB}_{6}$ exhibit a different behavior with respect to changes in the exchange mixing strength. The $4 f$-projected DOSs of $\mathrm{NdB}_{6}$ show a three-peak structure with a fractionally occupied $4 f$ level at the Fermi energy. This is in accordance with the spectral function of the effective non-interacting system of the HartreeFock approximation to our model for a fractional occupancy of the impurity. Hence, the positions of the $4 f$ levels in our DFT simulations for different values of the exchange mixing are expected to follow the curves (5.176) - (5.179) as obtained for a fractional occupancy of the impurity in our model. Note that the response of the $4 f$ orbital energies to changes in the exchange mixing strength is not linear in the DFT data. For example, the fully occupied $4 f$ levels of $\mathrm{NdB}_{6}$ show a less-than-linear decrease in the energy relative to the Fermi level. The deviation from a linear curve can be rationalized by the fact that a larger exchange mixing reduces the energy of the $4 f$ levels, leading to a larger fractional occupancy of the $4 f$ shell, and hence result in a smaller decline of the occupied levels with respect to the Fermi level. In our simulations of $\mathrm{NdB}_{6}$ such a gradual increase of the fractional occupancy of the $4 f$-shell from $N_{f}=3.57$ at an exchange mixing factor of 0.05 to $N_{f}=3.69$ at an exchange mixing factor of 0.20 is observed. We account for this increase in the $4 f$ occupancy by replacing the fractional occupancy in eqs. (5.176) $-(5.179)$ with a linear fit to the $n_{f}^{\text {frac }}(\eta)$ data. We see in fig. 5.12 that the DFT data and the curves of our model are in good agreement.

Because the fractionally occupied state is pinned to the Fermi level, the energies of the fully occupied and empty orbitals are computed relative to the fractionally occupied orbital. The FLL term that leads to a linear upwardshift in the curves for integer occupancies is independent of the occupancy of the individual orbitals. Hence, the fully occupied, fractionally occupied, and empty levels are affected equally and the shift is subtracted out in the curves (5.176) - (5.179) for the case of a fractional occupancy of the $4 f$ shell.

Note that in our model the double counting term does not counteract the splitting of the single-particle energy levels coming from the exact exchange contribution. I. e. for integer and fractional occupancies, respectively, still a two-peak shape and a three-peak shape of the spectral function is found. Moreover, the double counting term does not reduce the peak distance between the occupied and unoccupied orbitals. The self-interaction correction due to the exact exchange term stays in effect. However, if a share of the double counting correction is FLL-like, all features of the spectral function of the localized orbitals are equally shifted to higher energies.

The results of our model calculations allow us to propose a simple way to 
5 The density of states of localized orbitals with fractional occupancy: study of a model system

obtain a corrected one-particle excitation spectrum of the material by reconstructing an approximate ensemble of Slater determinants based on information that is known about the system. In the following we present an example of such an approach which we use as a "rule of thumb" to obtain a better approximation to the spectral function of the $4 f$ orbitals of $\mathrm{NdB}_{6}$.

In the DFT simulations of $\mathrm{NdB}_{6}$, besides a contribution of the boron bands, the main contribution to the DOS at the Fermi level comes from the partially occupied $\mathrm{Nd} 4 f$ state. Let us assume that only the $4 f$-projected DOS is corrupted. We can then use the results of the model system to estimate the correct $4 f$ energy levels as follows. Let $\Delta E_{\text {peak }}$ denote the energy separation between the energy of the unoccupied orbitals and the energy of the fully occupied orbitals of the majority spin direction in the $4 f$-projected DOS. According to eq. (5.141), $\Delta E_{\text {peak }}$ is a measure for the screened effective interaction strength $U-J$ within the $4 f$ shell. For a fractional occupancy of the impurity, the spectral function from the many-body approach to the model has a feature in the majority spin direction at the Fermi level. Further, a feature of occupied states is located at an energy $U-J$ below the Fermi level. If we transfer this result to the DOS of $\mathrm{NdB}_{6}$, we can estimate the correct energy level of the fully occupied $4 f$ states at an energy of $\Delta E_{\text {peak }}$ below the Fermi level. This gives the following corrected energies for the occupied $4 f$ levels:

\begin{tabular}{c|cccc}
$\eta^{\text {DFT }}$ & 0.05 & 0.10 & 0.15 & 0.20 \\
\hline$\left[E_{\mathrm{F}}-\Delta E_{\text {peak }}\left(\eta^{\text {DFT }}\right)\right] / \mathrm{eV}$ & -1.4 & -2.8 & -4.2 & -5.6
\end{tabular}

Since the effect of the self-interaction correction due to the exchange mixing in the PBE0r hybrid functional grows linearly with $\eta^{\mathrm{DFT}}$, the energy of the occupied $4 f$ levels in the above data decreases linearly with $\eta^{\text {DFT }}$. In the experimental photoemission spectrum of Patil et al.[30] the occupied $4 f$ levels are found at an energy of $-6.3 \mathrm{eV}$. We find the same value when extrapolating the above data at an exchange mixing of $\eta^{\mathrm{DFT}}=0.225: E_{\mathrm{F}}-\Delta E_{\text {peak }}\left(\eta^{\mathrm{DFT}}=\right.$ $0.225)=-6.3 \mathrm{eV}$. We remark that this exchange mixing factor also gives the position of the occupied $4 f$ levels in the $\mathrm{DOS}$ of $\mathrm{PrB}_{6}$ in accordance with photoemission experiments. For $\mathrm{PrB}_{6}$ it is possible that, due to their degeneracy, both the TYPE II and the (corrected) TYPE III state are realized in a real material. The above rule of thumb could prove to be useful to correct the DOS also for DFT+PBE0r hybrid functional simulations of other metallic materials with localized orbitals.

A DOS with $4 f$ states at the Fermi level might also be an explanation for the unusual spectral features close to the Fermi level which are found in the photoemission spectra of $\operatorname{PrB}_{6}$ and $\mathrm{NdB}_{6}$ by Patil et al.[30]. For $\operatorname{PrB}_{6}$ the $4 f^{2} \rightarrow 4 f^{1}$ ionization peak is found at $E_{\mathrm{F}}-5 \mathrm{eV}$ and for $\mathrm{NdB}_{6}$ the $4 f^{3} \rightarrow 4 f^{2}$ ionization peak is found at $E_{\mathrm{F}}-6.3 \mathrm{eV}$. With a screened interaction of $\sim 6 \mathrm{eV}$ in these materials [133] further quasiparticle levels can be expected in the vicinity of the Fermi level. These levels can hybridize with metallic states of the system and produce a fractional occupancy of the $4 f$ shell, and, moreover, lead to features in the $4 f$ projected spectral function close to the Fermi level. For $4 f$ states slightly above the Fermi level that hybridize with metallic states, the fractional occupancy can expected to be larger the closer the $4 f$ states are to the 
Fermi level. This might explain a rather large fractional $4 f$ occupancy in $\mathrm{NdB}_{6}$ and a possibly small fractional $4 f$ occupancy in $\operatorname{PrB}_{6}$. To clarify the emergence of the spectral features, it would certainly be interesting to revisit $\operatorname{PrB}_{6}$ and $\mathrm{NdB}_{6}$ in a theoretical study with a combination of DFT with the PBE0r hybrid functional and a Green's function-based approach. For example, our model can be extended to an Anderson impurity model (AIM) by including an explicit hybridization between the impurity and the bath. For the AIM, the spectral function can be computed by means of a continuous-time quantum Monte Carlo algorithm (see e.g. [154, 155]) and the maximum entropy method [156]. The parameters for the AIM can be obtained from the DFT+PBE0r data. Another possibility is to use a full DFT+DMFT approach, see [97-101]. Both of the suggested approaches offer the advantage that the spectral function is computed for an interacting system in a many-body approach. This is in contrast to the DFT+PBE0r approach, where we obtain the spectral function of an effective non-interacting system. However, solving the AIM with a continuous-time quantum Monte Carlo algorithm is computationally more expensive. Moreover, in the DFT+DMFT approach, the double-counting correction becomes more complicated because also a double counting of the correlation term needs to be taken into account.

\subsection{Summary}

In this chapter we rationalize the shape of the $4 f$-projected DOS of our DFT simulations of the rare-earth hexaborides $\mathrm{CeB}_{6}, \mathrm{PrB}_{6}$, and $\mathrm{NdB}_{6}$ with the PBE0r hybrid functional. For this purpose, we set up a model system consisting of a non-interacting bath and an impurity of localized orbitals. The localized orbitals are subject to an on-site interaction. An exchange of particles between the bath and the impurity is allowed. This model system represents a simple analog to the situation in the rare-earth hexaborides, with the localized orbitals representing the rare-earth $4 f$ orbitals and with the bath representing the metallic states in the vicinity of the Fermi level. Notably, the localized orbitals comprise an open subsystem of the full model so that a fractional occupancy of the localized orbitals is possible. The impurity is modeled such that the eigenstates to the interacting impurity Hamiltonian are Slater determinants, which likewise are eigenstates to the effective non-interacting Hamiltonian of the impurity in the Hartree-Fock approach.

We analyze the model in two different approaches: in a full many-body treatment and in the Hartree-Fock approximation. In particular, for the ground state of the model we compare the spectral functions of the localized orbitals of the original interacting system and of the effective non-interacting system of the Hartree-Fock treatment. The spectral functions coincide for integer occupancies of the impurity in the ground state but differ for fractional occupancies. The reason for this is traced back to the dependence of the energies of the localized orbitals in the effective non-interacting system of the Hartree-Fock approach on the orbital occupancies and is summarized in the following.

If, in the Hartree-Fock approach, the total energy of the full model system is 
minimized by an integer total occupancy number of the impurity, the occupancy numbers of the individual localized orbitals are either 0 or 1 and the impurity state can be represented in terms of a single Slater determinant. In this case a localized orbital can take one of two different energies, depending on the occupancy. The energy level for occupancy 0 represents the energy associated with an excitation of the ground state of the original interacting model system through the insertion of a particle. Conversely, for occupancy 1, the energy level represents the energy associated with an excitation of the ground state of the original interacting model system through the removal of a particle. If the total energy of the model system is minimized by a fractional total occupancy number of the impurity subsystem, this open subsystem must be represented by an ensemble of Slater determinants. In this case there are three different energy levels of the localized orbitals in the ground state, which are associated with the occupancies 0,1 , and a fractional occupancy $0<n_{f}^{\text {frac }}<1$. The energy level which is fractionally occupied is pinned to the Fermi level. It is pointed out that these energy levels do not represent the energies associated with single-particle excitations of the original interacting model system. Hence, the spectral function which represents the one-particle excitation spectrum of the interacting model is correctly reproduced by the Hartree-Fock DOS for an integer ground state occupancy, but this is not the case for a fractional ground state occupancy.

The spectral function of the effective non-interacting system, which represents the impurity in the Hartree-Fock approach to the model, matches the DOSs from the DFT simulations of $\mathrm{CeB}_{6}, \mathrm{PrB}_{6}$, and $\mathrm{NdB}_{6}$ with the PBE0r hybrid functional qualitatively for both integer and fractional occupancies of the impurity. Moreover, the Hartree-Fock DOS of the model and the DFT DOSs show the same behavior with respect to changes in the fractional occupancy of the localized orbitals and with respect to changes in the strength of the exchange mixing. Based on the correspondence between the model system in Hartree-Fock approximation and the Kohn-Sham system in DFT with the PBE0r hybrid functional, the mismatch between experimental photoemission spectra and our DFT DOSs is rationalized as follows.

If, in DFT, we apply the PBE0r hybrid functional as the functional of exchange and correlation, the total energy receives an admixture of exact exchange. Moreover, the energies of the Kohn-Sham states receive a contribution coming from the exact exchange term. The exchange contribution is obtained in a local basis of single-particle states of the individual atoms within the material, onto which the Kohn-Sham states are projected. A projection of the Kohn-Sham states onto the local basis states can result in a fractional occupancy of the local basis states. For the rare-earth hexaborides the states of the local basis for the rare-earth ion include the $4 f$ orbitals, which are close to the Fermi level and can receive a fractional occupancy. Hence, we have an analogy between the situation in DFT with the PBE0r hybrid functional and our model system. In both cases we aim to calculate properties of a system of interacting particles by the treatment of an effective non-interacting auxiliary system. Each of the basis states of the effective non-interacting system is associated with an energy level that depends on the possibly fractional occupancies of one-particle 
basis states due to an exchange contribution. Based on the analogy between the DFT system and the model we come to the following conclusion. In the case of a fractional occupancy of localized orbitals the Kohn-Sham energies, which are associated with the auxiliary non-interacting Kohn-Sham states, do not correctly represent the one-particle excitation spectrum of the material for excitations of the ground state through particle insertion and particle removal.

This shows that the combination of DFT with the PBE0r hybrid functional in the current implementation can produce a DOS that is not in agreement with experimental spectra. Nevertheless, the use of the PBE0r hybrid functional is justified. On the one hand, it improves over the results from simulations with the PBE functional. Importantly, a better compensation of the unphysical selfinteraction present in the Hartree interaction is achieved by the PBE0r hybrid functional. On the other hand, we know that the DOS from DFT can be a poor approximation to the one-particle spectra of metallic materials with localized orbitals in which a fractional occupancy of the ground state is possible. Hence, such systems have to be approached with care. For our simulations of the rare-earth hexaborides we propose a "rule of thumb" that allows to estimate a spectral function for the $4 f$ orbitals in materials in which the $4 f$ orbitals receive a fractional occupancy in the ground state. 



\section{DFT surface simulations of lanthanum hexaboride}

The scientific work presented in this chapter is published in ref. [157]:

P. Buchsteiner, F. Sohn, J. G. Horstmann, J. Voigt, M. Ciomaga Hatnean, G. Balakrishnan, C. Ropers, P. E. Blöchl, and M. Wenderoth. "Surface resonance of the $(2 \times 1)$ reconstructed lanthanum hexaboride (001)-cleavage plane: A combined STM and DFT study", Physical Review B 100, 205407 (Nov 2019)

DoI: $10.1103 /$ PhysRevB. 100.205407

URL: https : //journals .aps .org/prb/abstract/10.1103/ PhysRevB. 100.205407

In section 2.4, it was pointed that in prior experimental investigations of the $\mathrm{LaB}_{6}(001)$ surface a $(1 \times 1)$ arrangement of lanthanum ions at the surface level was found. Sample preparations in these studies relied on polishing and heating the sample. It is plausible that these preparation techniques can lead to a preferential surface structure. The recent theoretical prediction of a stable $(2 \times 1)$ structure on the $\mathrm{LaB}_{6}(001)$ surface accentuated the need for new research on the surface at temperatures far below the thermionic emission regime and with sample preparation techniques other than those used in prior studies. An alternative way to prepare a sample surface is the cleaving of a single crystal, which exposes the cleavage plane as a pristine surface for experimental investigations. $\mathrm{LaB}_{6}$ does not have a natural cleavage plane and $a$ priori several cleaving outcomes seem possible. For example, a result could be a purely lanthanum terminated surface on one side and a surface terminated by completely intact boron octahedra on the other side. Another possibility would be a "saw tooth" structure, where on both surfaces only each second surface site is occupied by either lanthanum ions or boron octahedra. In principle, the cleaving could also proceed through the boron octahedra.

In this chapter we present the results of a joint research project with the purpose to determine and explain the atomic and electronic structure of the $\mathrm{LaB}_{6}$ (001) surface, prepared by cleaving at room temperature and UHV conditions. We investigated the $\mathrm{LaB}_{6}(001)$ cleavage plane on the basis of DFT simulations, while related experimental research was conducted by our collaborators. The experimental side comprises the fabrication of $\mathrm{LaB}_{6}$ single crystals and surface investigations via scanning tunneling microscopy/spectroscopy (STM/STS), as well as atomic force microscopy (AFM), and low-energy electron diffraction (LEED). 
This chapter is organized as follows: First, we give an overview of the organization of the joint research project and list our scientific collaborators. Second, we provide a summary of the findings of our experimental colleagues. This is followed by a presentation of our theoretical data. Subsequently, we discuss our results and link experimental and theoretical findings.

\subsection{Statement on the scientific collaboration}

Multiple people of different work groups contributed to our joint research project on the $\mathrm{LaB}_{6}$ (001) surface. On the experimental side, AFM as well as STM and STS measurements on the $\mathrm{LaB}_{6}$ samples have been conducted by Philipp Buchsteiner and Jan Voigt in the group of Martin Wenderoth at the University of Göttingen. The $\mathrm{LaB}_{6}$ single crystal specimen have been prepared by Monica Ciomaga Hatnean in the group of Geetha Balakrishnan at the University of Warwick. LEED investigations of the surface were performed by Jan G. Horstmann in the group of Claus Ropers at the University of Göttingen.

For the theoretical part, simulations based on density functional theory (DFT) and the processing of the DFT data have been performed by the author in the group of Peter E. Blöchl at the University of Göttingen and the Clausthal University of Technology.

\subsection{Experimental measurements on cleaved $\mathrm{LaB}_{6}$ surface samples}

In this section we recapitulate the findings of our experimental collaborators on the $\mathrm{LaB}_{6}(001)$ cleavage plane, which are presented in the experimental part of our joint publication, ref. [157].

\subsubsection{Experimental methods}

For the experimental studies, rods of single-crystalline $\mathrm{LaB}_{6}$ have been grown with the floating zone technique $[158,159]$. Small single crystal samples have been prepared by cutting the $\mathrm{LaB}_{6}$ boules along the $(001)$ crystal planes to rectangular samples of approximate size $1 \mathrm{~mm} \times 4 \mathrm{~mm} \times 300 \mu \mathrm{m}$. Before an STM measurement, a sample was cleaved in-situ along the (001) plane at room temperature and UHV conditions and transferred to the STM head after being cooled to cryogenic temperature. The STM measurements were conducted at a temperature of $8 \mathrm{~K}$ and in UHV conditions with a base pressure of $4 \times 10^{-11}$ mbar. STM tips were fabricated from polycrystalline tungsten wire. In addition to the STM measurements, the cleavage plane was studied by AFM at ambient conditions. Furthermore, samples of the $\mathrm{LaB}_{6}(001)$ cleavage plane were subject to LEED investigations. For the LEED studies, an ultrafast LEED (ULEED) setup was used, which has a small beam diameter of $80 \mu \mathrm{m}$ at the sample and a smaller electron count within the beam in comparison to a conventional LEED apparatus. Details on the ULEED apparatus can be found 
in ref. [160]. For the experiments in the ULEED the samples were cleaved in-situ at a base pressure of $2 \times 10^{-8}$ mbar and investigated at a pressure of $2 \times 10^{-10}$ mbar.

\subsection{2 | Surface morphology}
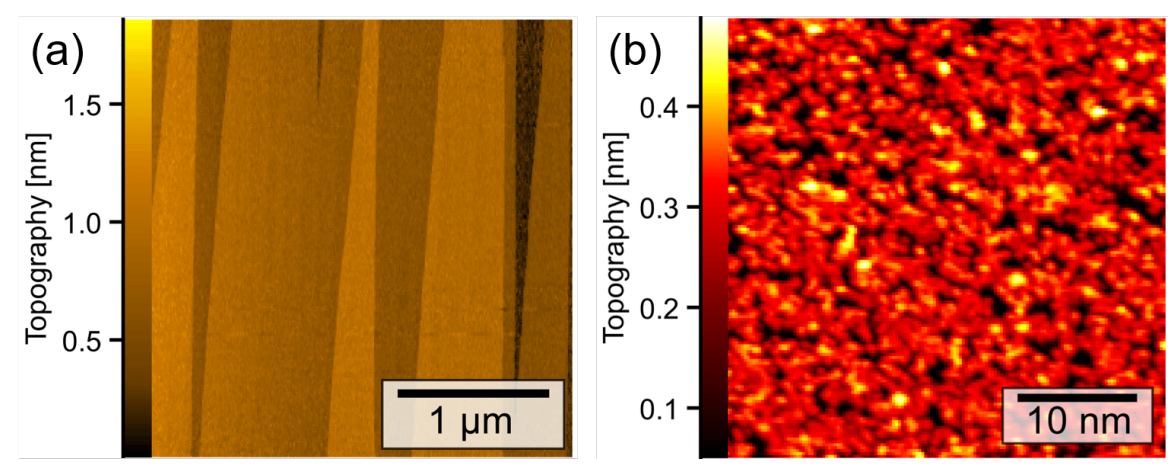

Figure 6.1: (a) Large-scale topography image of the $\mathrm{LaB}_{6}$ (001) surface recorded via AFM. All steps heights are of integer multiples of the lattice constant. (b) STM CCT showing a disordered area of the surface. Bias voltage: $1 \mathrm{~V}$, tunneling current: $0.1 \mathrm{nA}$. Figure originally published in [157]. Reprinted with permission from the American Physical Society. (C) (2019) American Physical Society.

The large-scale AFM topography image in fig. 6.1(a) shows, that the $\mathrm{LaB}_{6}$ (001) cleavage plane exhibits numerous flat terraces that are separated by steps with heights of integer multiples of the lattice constant. STM constant current topography (CCT) images often show a disordered surface, see fig. 6.1(b). However, a few areas have been found, where CCTs reveal an atomically ordered surface as depicted in figs. 6.2 and 6.3. The atomically ordered areas exhibit a chainlike structure with a chain separation of two bulk lattice constants (cf. fig. 6.3(b)). Commonly, the chains are rather short with lengths of a few nanometers and are rarely arranged in parallel. Instead the surface mostly has a labyrinthlike appearance with numerous defects as seen in the blue-framed area of fig. 6.2. Occasionally, surface areas exhibit longer, parallel chains, which reach lengths of more than $10 \mathrm{~nm}$. This is the case for the red-framed surface region of fig. 6.2 , for which $(2 \times 1)$-reconstruction spots can be identified in the Fourier transform (red circles). In contrast, although individual chains can be identified in the blue-framed area of fig. 6.2, its Fourier transform does not show distinct spots that can be assigned to a $(2 \times 1)$ reconstruction because of the lack of long-range order.

The most ordered $(2 \times 1)$ surface area, exhibiting long parallel chains, is found in the vicinity of a step edge, where the chains are aligned perpendicular to the step edge. Moving away from the edge, the arrangement in long parallel chains eventually breaks up and the long-range order is lost. These experimental observations suggest, that the $(2 \times 1)$ reconstruction with long-range order occurs preferentially close to step edges. 


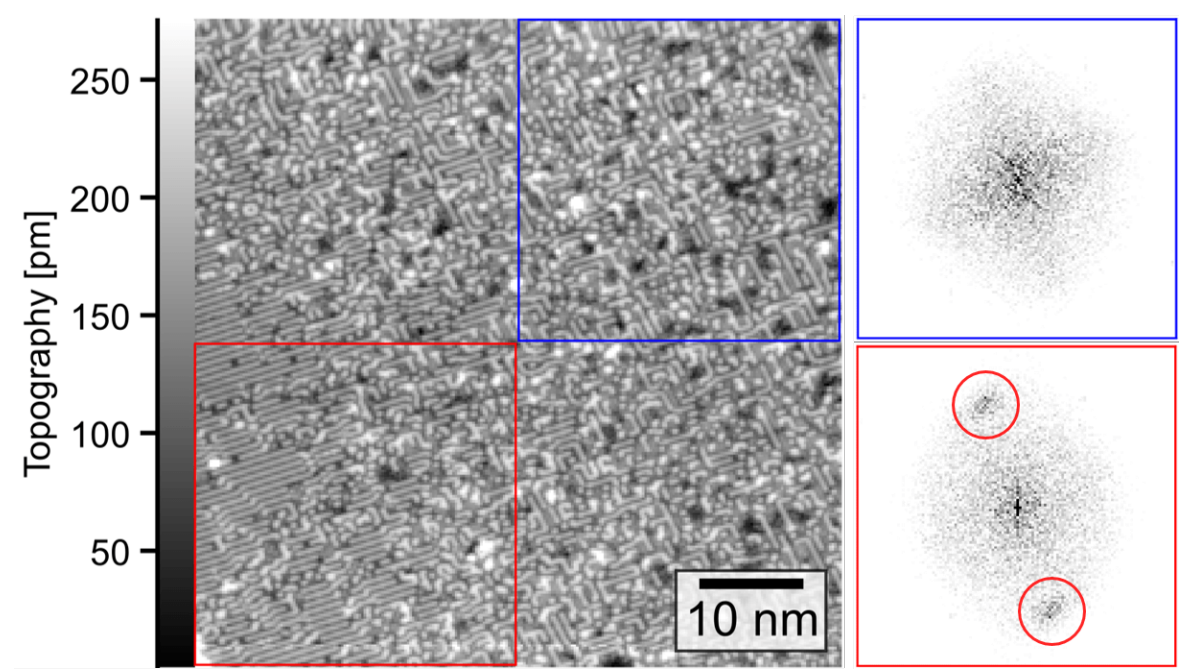

Figure 6.2: STM CCT image of an area of the $\mathrm{LaB}_{6}$ (001) surface, showing a $(2 \times 1)$ reconstruction. The two colored frames on the right contain the Fourier transforms of the same-color framed regions of the CCT. Bias voltage: $0.8 \mathrm{~V}$, tunneling current: $0.1 \mathrm{nA}$. Figure originally published in [157]. Reprinted with permission from the American Physical Society. () (2019) American Physical Society.

The appearance of disorder in the CCT images can have multiple reasons. In a study of $\mathrm{SmB}_{6}$ by Rößler et al. [161], cleaving at $20 \mathrm{~K}$ produced atomically flat surface areas, while no such surface was observed after cleaving at room temperature. Hence, it is plausible that for $\mathrm{LaB}_{6}$, cleaving at room temperature could lead to less ordered surfaces, than cleaving at cryogenic temperatures. Although the pressure was very low, another reason for disorder in the CCT images could be residual gas that is adsorbed to the surface and attaches to the boron dangling bonds. This could affect the surface especially, when the cleaving proceeds through boron octahedra, which could lead to several exposed boron dangling bonds. Moreover, the quality of the STM tips has a significant influence on the quality of the CCT images and a blunt tip is less likely to resolve the individual chains.

When studying the surface morphology of the cleavage plane in a conventional LEED setup, a $(1 \times 1)$ LEED pattern was observed, that vanished after 30 minutes of measurement time under UHV conditions. Further measurements have been performed in the ULEED apparatus, where a $(1 \times 1)$ LEED pattern was also observed. Moreover, in the ULEED in certain surface areas additionally $(2 \times 1)$ and/or $(1 \times 2)$ LEED patterns appeared, see fig. 6.4. The ULEED electron beam appears to be less surface altering, as no apparent change in the LEED pattern was observed after several hours of measurement time.

Besides the steps of heights of integer multiples of the lattice constant, also a step of a fraction of the lattice constant has been observed in an STM CCT as shown in fig. 6.5. On the corresponding surface, no surface structure could be resolved at the chosen positive bias voltage. This is interpreted as a region of the surface, with a different termination than the usually observed ones. 

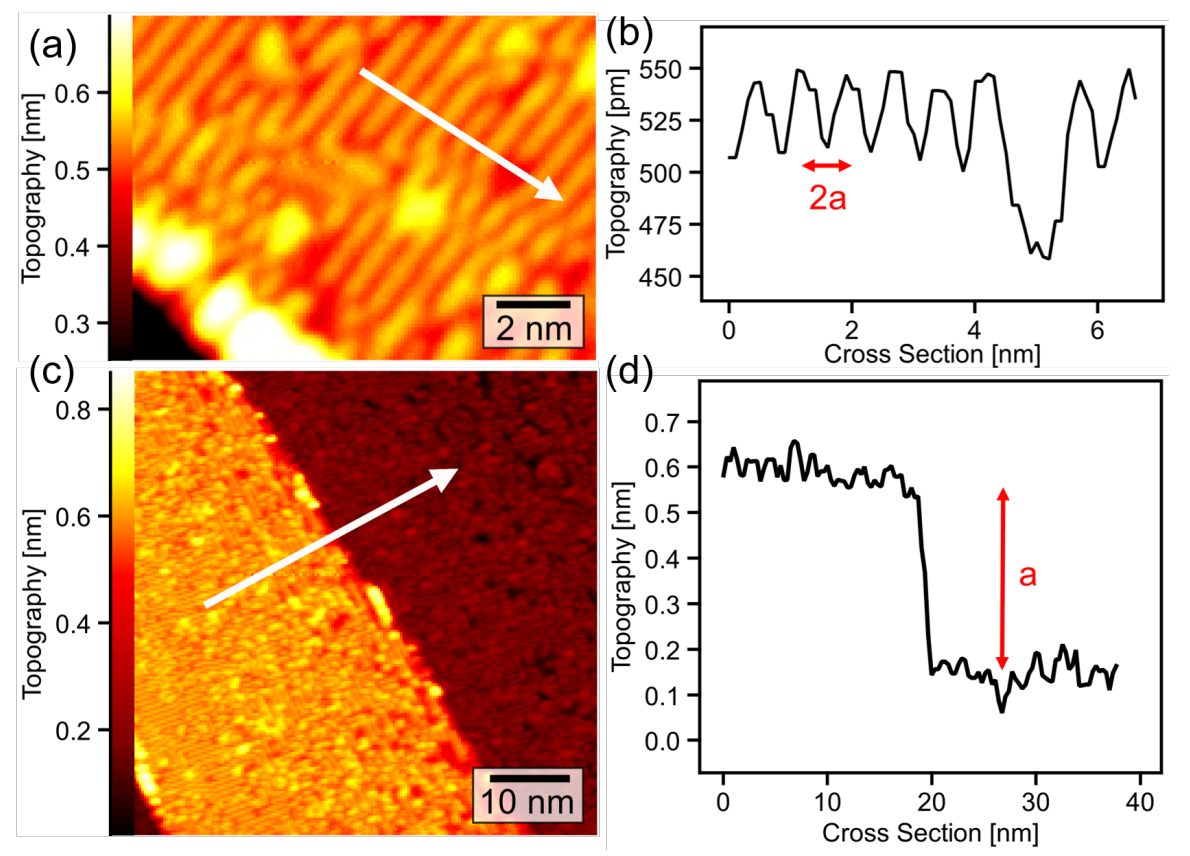

Figure 6.3: (a) STM CCT image of a $(2 \times 1)$ reconstructed area of the $\mathrm{LaB}_{6}$ (001) cleavage plane at high resolution. Bias voltage: $1 \mathrm{~V}$, tunneling current: $0.1 \mathrm{nA}$. (b) Topographic height profile along the arrow indicated in (a). The distance between two neighboring rows is two lattice constants. (c) STM CCT image of two $(2 \times 1)$ surface areas separated by a step. Bias voltage: $1 \mathrm{~V}$, tunneling current: $0.1 \mathrm{nA}$. The area depicted in (a) is in the bottom left corner of (c). (d) Height profile along the arrow indicated in (c). The step height is one lattice constant. Figure originally published in [157]. Reprinted with permission from the American Physical Society. (C) (2019) American Physical Society. 
(a)

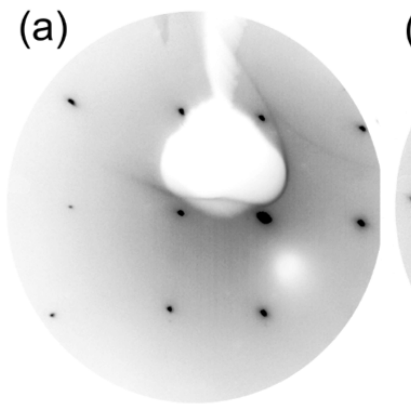

(c)

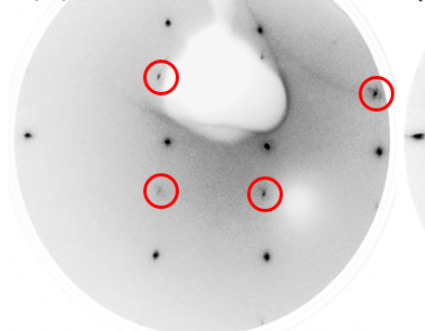

(b)

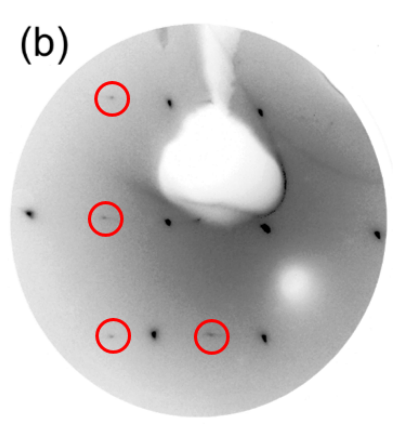

(d)

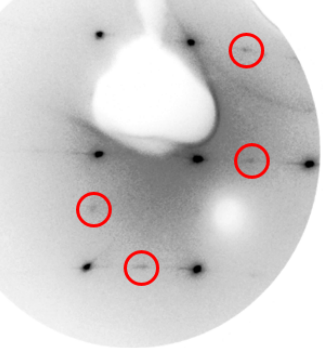

Figure 6.4: LEED patterns of four different areas of the $\mathrm{LaB}_{6}(001)$ cleavage plane, recorded with the ultra-fast LEED setup. $(1 \times 1)$ spots are found in all LEED patterns. $(2 \times 1)$ and $(1 \times 2)$ spots are encircled red. (a) $(1 \times 1)$ pattern at $130 \mathrm{eV}$ and room temperature. (b) Additional $(2 \times 1)$ pattern at $100 \mathrm{eV}$ and room temperature. (c) Additional $(1 \times 2)$ pattern at $100 \mathrm{eV}$ and $27 \mathrm{~K}$. (d) Additional $(2 \times 1)$ and $(1 \times 2)$ patterns at $100 \mathrm{eV}$ and $27 \mathrm{~K}$. Figure originally published in [157]. Reprinted with permission from the American Physical Society. ( (2019) American Physical Society.
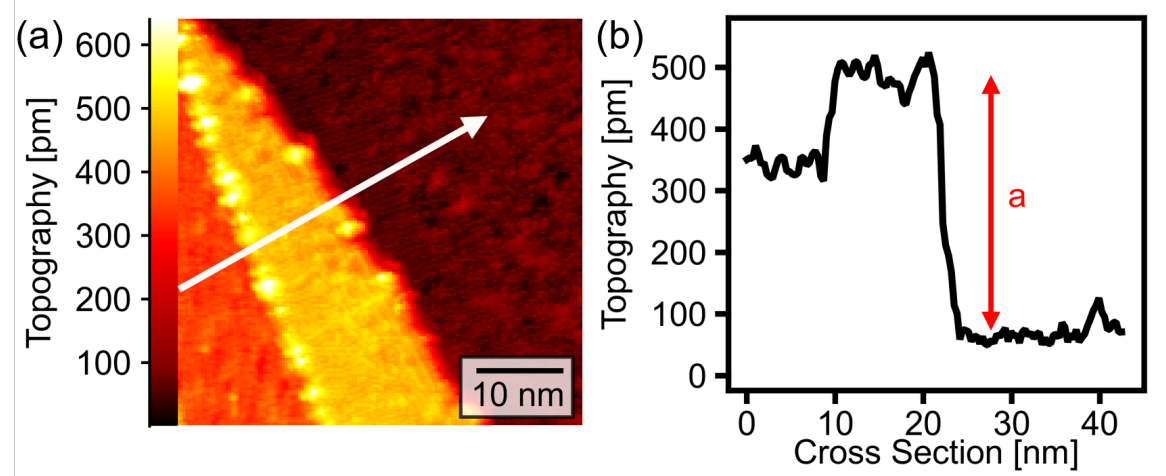

Figure 6.5: (a) Large scale STM CCT of a surface region exhibiting two steps. Bias voltage: $0.8 \mathrm{~V}$, tunneling current: $0.1 \mathrm{nA}$. (b) The height profile along the arrow in (a), one step is of height $\sim 1.3 \AA$, the other of one lattice constant. All STM data by P. Buchsteiner, J. Voigt and M. Wenderoth. Figure by P. Buchsteiner, originally published in [157]. Reprinted with permission from the American Physical Society. ( (2019) American Physical Society. 


\subsubsection{Scanning tunneling spectroscopy}

In the previous section we saw that in constant current topographies the $\mathrm{LaB}_{6}$ (001) cleavage plane exhibits a chainlike $(2 \times 1)$ reconstruction. Mostly, the chains are rather short and are arranged in a labyrinthlike structure. In the vicinity of step edges also longer chains with a parallel alignment have been observed.
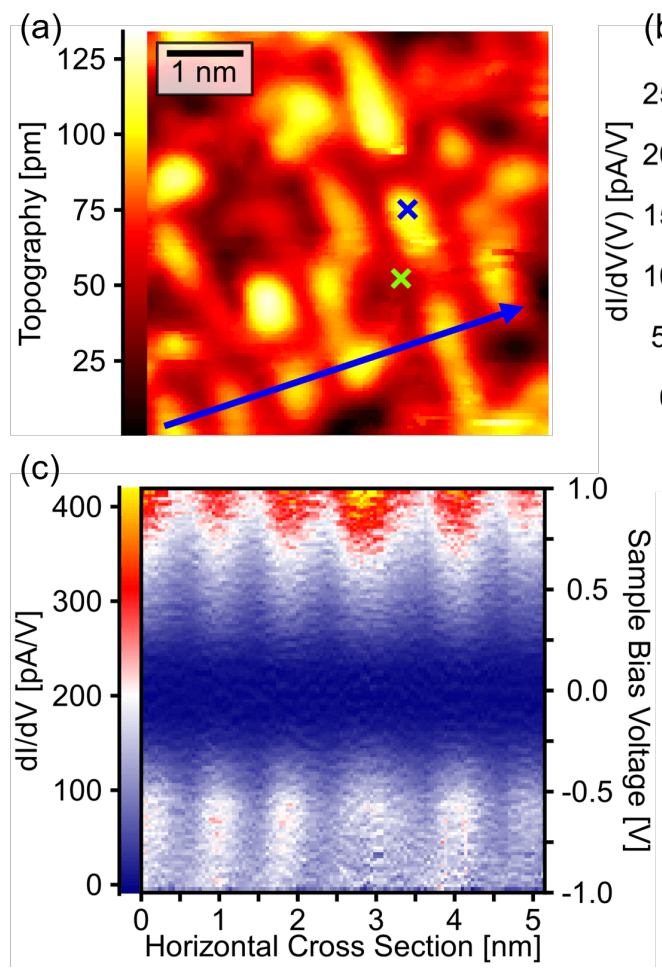

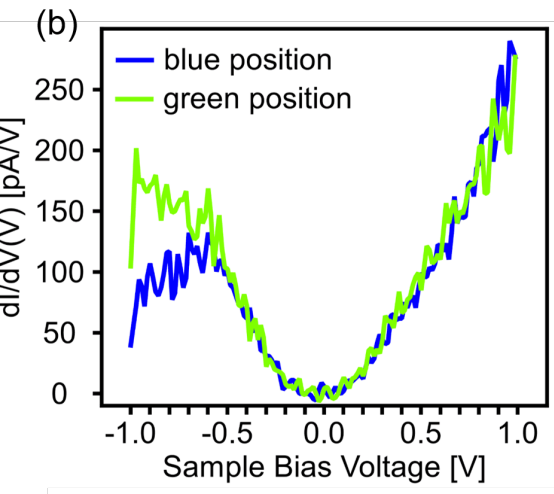

(d)

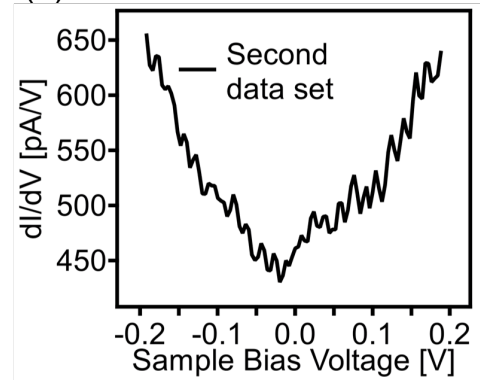

Figure 6.6: (a) CCT of a surface area, that was selected for STS measurements. Bias voltage: $1 \mathrm{~V}$, tunneling current: $0.1 \mathrm{nA}$. Tunneling spectra taken at the marked positions are depicted in (b). (c) spatially resolved tunneling spectrum along the blue arrow in (a). (d) average tunneling spectrum from a different data set. More information is given in appendix E.2 and fig. E.2. Figure by P. Buchsteiner, originally published in [157]. Reprinted with permission from the American Physical Society. (C) (2019) American Physical Society.

For the investigation of the electronic properties and to clarify the underlying atomic structure, the $(2 \times 1)$ surface reconstruction was subject to scanning tunneling spectroscopy (STS) measurements. Figure 6.6(a) shows the CCT of a surface area, for which quasi-simultaneously to the CCT at each measurement point a tunneling spectrum was recorded. I. e. at each measurement point the tip height is first adjusted by the feedback loop and subsequently a STS measurement is performed with the feedback loop being temporarily deactivated. In fig. 6.6(b) we show the tunneling spectra obtained from a position on top of a protrusion (blue marked position) and from a lower laying part of the CCT in the vicinity of the protrusion (green position). For negative bias voltages the 
spectra exhibit a feature at $-0.6 \mathrm{~V}$. At positive bias voltages both spectra rise steeply.

For a more detailed analysis of variations in the $\mathrm{d} I / \mathrm{d} V$ data for small changes of the tip position, tunneling spectra from multiple positions along a defined line can be combined into a tunneling spectrum $\mathrm{d} I / \mathrm{d} V(V, x)$ with a spatial dependence $x$. Because the individual spectra were recorded quasi-simultaneously to the CCT, the initial tip positions for these spectra depend on the topographic height profile along the defined line. To project the spatially resolved data on a line of constant height, a topography normalization is applied to the raw STS data. More details on this topography normalization are given in appendix E. The spatially resolved $\mathrm{d} I / \mathrm{d} V(V, x)$ data along the blue arrow in fig. 6.6(a) is depicted color-coded in fig. 6.6(c). It shows increased $\mathrm{d} I / \mathrm{d} V$ values above the topographic protrusions for a bias voltage of $-0.6 \mathrm{~V}$. Furthermore, the position of the protrusions along the arrow in the CCT also coincides with the position of the $\mathrm{d} I / \mathrm{d} V$ maxima at large bias voltages.

The reader might have noticed, that the $\mathrm{d} I / \mathrm{d} V$ curves in fig. 6.6(a) and (c) drop to zero at small bias voltage. This behavior is not to be interpreted as a non-metallic surface, but rather as a collapse of the tunneling contact caused by the reduction of the bias voltage. To resolve the spectrum in the small bias voltage regime, a spectrum from a second data set is shown in fig. 6.6(d). For this spectrum the initial bias voltage was set to a smaller value of $0.2 \mathrm{~V}$ while the tunneling current target was kept at $0.1 \mathrm{nA}$. This results in an expected tip position closer to the surface than in the previous measurements. For these parameters a stable non-zero tunneling current is maintained throughout the measurement and the $\mathrm{d} I / \mathrm{d} V$ remains larger than zero. Hence, the second data set shows that the surface is indeed metallic. Above the minimum up to $0.1 \mathrm{~V}$ bias voltage, the raised shape of the spectrum indicates a feature. Additional information on this STS measurement is given in appendix E.

\subsection{Surface simulations}

In experimental scanning tunneling microscopy (STM) constant current topography (CCT) images of the $\mathrm{LaB}_{6}(001)$ cleavage plane, labyrinthlike and chainlike patterns were found in atomically flat surface areas. Moreover, scanning tunneling spectroscopy (STS) measurements revealed a prominent feature at a bias voltage of $-0.6 \mathrm{~V}$ and a second feature at small positive bias voltage between $0.0 \mathrm{~V}$ and $0.1 \mathrm{~V}$. To clarify the atomic structure of the surface and to resolve the precise composition of the surface's electronic structure, we performed simulations on the $\mathrm{LaB}_{6}(001)$ surface, based on density functional theory (DFT).

In this section we present the results of our theoretical study, which include an analysis of the atomic structure of the surface, a discussion of the density of states of the surface, simulated STM topography images, and a simulated ST spectrum. We compare our results with the experimental data and show that the previously suggested atomic structure, with terminal lanthanum ions arranged in rows [31], indeed leads to the chainlike patterns in the STM topographies. 
Furthermore, we show that by tunneling from the tip into the unoccupied surface states predominantly lanthanum $d_{3 z^{2}-r^{2}}$ states are addressed. Below the Fermi level we find a dominant contribution of boron $s p$-hybrid dangling bonds with a distinct surface resonance.

\subsubsection{Simulation setup}
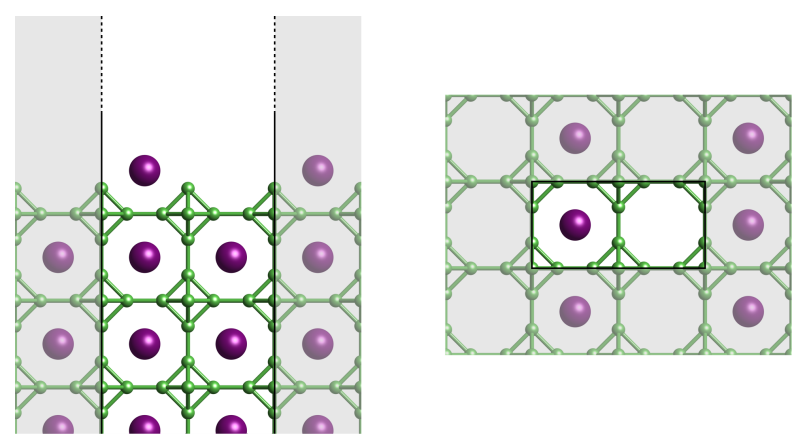

Figure 6.7: Side view (left) and top view (right) of the top part of the unit cell of the $\mathrm{LaB}_{6}(001)$ slab. $\mathrm{B}_{6}$ octahedra are colored green, La ions are shown in purple. The unit cell is indicated by the white background in between the left and right cell boundaries (black lines). Adjacent unit cells are grayed out. At the surface level only every second La row is occupied. The top view (right) shows only the topmost La ions and $\mathrm{B}_{6}$ octahedra. Figure originally published in [157]. Reprinted with permission from the American Physical Society. (C) (2019) American Physical Society.

As outlined in section 2.4, data from prior experimental studies indicate a lanthanum termination of the $\mathrm{LaB}_{6}(001)$ surface. Together with the occurrence of a $(2 \times 1)$ structure in the STM CCTs of the $\mathrm{LaB}_{6}(001)$ cleavage plane this supports the previous assumption of a stable $(2 \times 1)$ lanthanum terminated surface reconstruction [31]. Hence, we chose to simulate $\mathrm{LaB}_{6}(001)$ surface with terminal lanthanum chains. A setup for a DFT surface simulation follows the same basic principle of a bulk setup, in that it is based on a unit cell which is repeated in real space for all linear combinations of three 3-dimensional lattice vectors. However, the unit cell of a DFT surface simulation is constructed by stacking several bulk unit cells in one spatial direction and appending an empty vacuum space on both ends of the stack. The length of the corresponding lattice vector needs to be adjusted according to the size of the unit cell. In real space this leads to a periodically reoccurring slab structure with a slab thickness of several layers of bulk unit cells, where periodic images of the slab are separated by a vacuum. Furthermore, in the unit cell for a chainlike $(2 \times 1)$ reconstruction a second stack of bulk unit cells is added along a crystal axis parallel to the surface plane, resulting in two side-by-side columns of bulk unit cells. The chainlike surface termination is achieved by having lanthanum atoms at both ends of the first column, which are not present at the ends of the other column. A graphical representation of the surface supercell for the 
$(2 \times 1)$ reconstruction is given in fig. 6.7. For the lattice constant we chose the bulk value of $a_{\text {bulk }}=4.15 \AA$ [38-42].

Note that the surface slab has a bottom and a top surface, where the bottom surface needs to be a mirror version of the top surface to avoid a polarization of the slab. Moreover, the bulk volume in between the two surfaces needs to be sufficiently thick to ensure that both surfaces are independent of each other. For the simulation of the $(2 \times 1) \mathrm{LaB}_{6}(001)$ surface slab a thickness of three layers of boron octahedra constitutes a sufficiently large spatial separation of the top and bottom surfaces to avoid artifacts. However, we performed simulations of slabs with three, five, and seven layers of boron octahedra to ensure convergence of our results with respect to the slab thickness. The data that is presented in the following sections is obtained from the simulations with slabs of seven layers thickness. Further, a sufficiently large vacuum between the slab and its neighboring periodic images is required, so that a surface slab and its periodic image do not affect each other. We chose a vacuum thickness of $4 a_{\text {bulk }}=16.60 \AA$, which allows a maximum spatial extension of the surface states into the vacuum of $2 a_{\text {bulk }}=8.30 \AA$.

Further simulation parameters include a $\vec{k}$-point grid of $8 \times 4 \times 1 \vec{k}$-points. We selected a plane-wave cutoff of $50 \mathrm{Ry}$ for the wave functions and a plane-wave cutoff of $100 \mathrm{Ry}$ for the charge density. The number of projector functions was set to $(2,2,2,1)$ for the lanthanum $(s, p, d, f)$ states and to $(2,2,1)$ for the boron $(s, p, d)$ states. The exchange mixing factor in the PBE0r hybrid functional was set to $\eta=0.15$ for both lanthanum and boron.

\subsubsection{Surface structure after atomic relaxation}

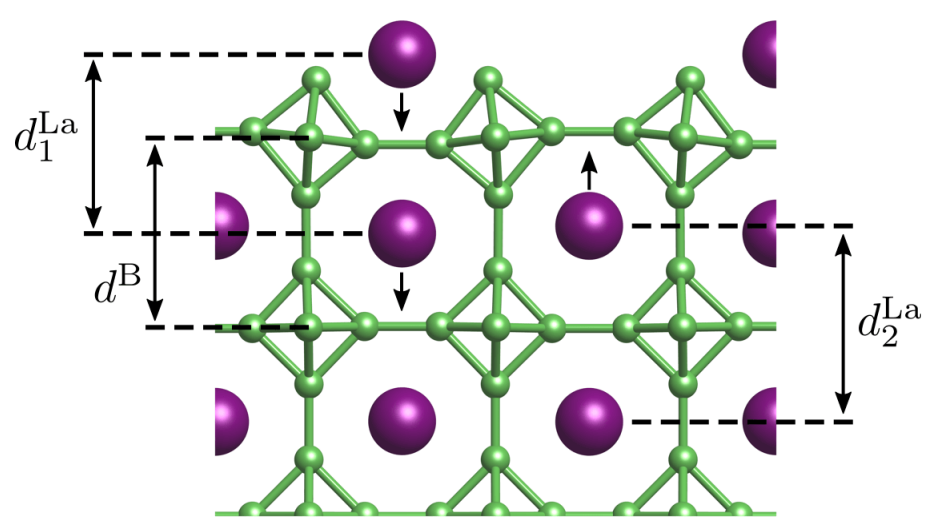

Figure 6.8: Side view of the topmost layers of the surface slab after atomic relaxation. Lanthanum ions are colored purple, boron octahedra are shown in green. Relaxation directions of the lanthanum ions from the initial position are indicated by arrows. Figure originally published in [157]. Reprinted with permission from the American Physical Society. (C) (2019) American Physical Society.

The atomic placement in the initial setup of the surface unit cell is derived 
from the atomic positions within the bulk structure. However, in the surface system two important symmetries are missing. First, the surface slab has a finite extension in one spatial direction and is exposed to the vacuum beyond. Second, the chainlike arrangement of the topmost lanthanum atoms changes the translational symmetry in the surface plane, perpendicular to the lanthanum chains. In the relaxed atomic structure of the slab unit cell the altered symmetries lead to a displacement of the atomic positions from their bulk analogs. A side view of the relaxed atomic structure in the upper layers of the surface slab is depicted in fig. 6.8.

In the topmost layer we find a tilting of the boron octahedra towards the lanthanum chains. Furthermore, we observe a displacement of the lanthanum ions and boron octahedra from their bulk positions in the direction perpendicular to the surface plane. A common measure for this displacement is the relative change in the distance $d$ between atoms in two neighboring layers with respect to the bulk lattice constant $a_{\text {bulk, }}$,

$$
\Delta=\frac{d-a_{\text {bulk }}}{a_{\text {bulk }}} .
$$

We find a relative change of $\Delta_{1}^{\mathrm{La}}=-4.3 \%$ for the lanthanum ion in the surface chain to the lanthanum ion below. The lanthanum ion below the empty lanthanum site at the surface moves towards the surface, with a relative change of $\Delta_{2}^{\mathrm{La}}=4.7 \%$ to the lanthanum ion beneath. Moreover, the center of mass distance of the boron octahedra in the two topmost layers increases by $\Delta^{\mathrm{B}}=1.4 \%$.

In addition to the above evaluation of the relative distance changes in a slab of seven layers of boron octahedra the analogous values were computed for a five layer slab. A maximum deviation of $0.007 \AA$ from our above results was found, which is insignificant in the context of our DFT simulations. Hence, we do not expect a difference in the surface structure for even larger slab thicknesses.

In a previous DFT study of the $(2 \times 1)$ reconstructed $\mathrm{LaB}_{6}(001)$ surface, Schmidt et al. reported relative atomic distance changes of $\Delta_{1}^{\mathrm{La}}=-6.2 \%$, $\Delta_{2}^{\mathrm{La}}=3.5 \%$, and $\Delta^{\mathrm{B}}=0.4 \%$ [31]. By comparison, our simulations resulted in slightly larger distances between the atoms of the topmost surface layers.

\subsubsection{Density of states}

Features in the density of states (DOS) that emerge due to the surface of the slab are identified in a side-by-side comparison of the bulk DOS and the DOS of the surface simulation. Such a comparison of the angular momentum-projected DOS of the $\mathrm{LaB}_{6}$ bulk and the $\mathrm{LaB}_{6}$ (001) surface is shown in fig. 6.9. The Fermi level is set to $E_{\mathrm{F}}=0 \mathrm{eV}$. According to the data $\mathrm{LaB}_{6}$ is a paramagnetic metallic system with $s$ and $p$ states of the boron cage prevailing below the Fermi level. Additionally, an admixture of lanthanum $d$ states is present in the occupied states. Above the Fermi level the DOS is dominated by lanthanum $d$ and $f$ states and exhibits a smaller contribution of the boron orbitals.

At $E_{\mathrm{F}}-0.2 \mathrm{eV}$ a feature is found in the surface DOS with no counterpart in 

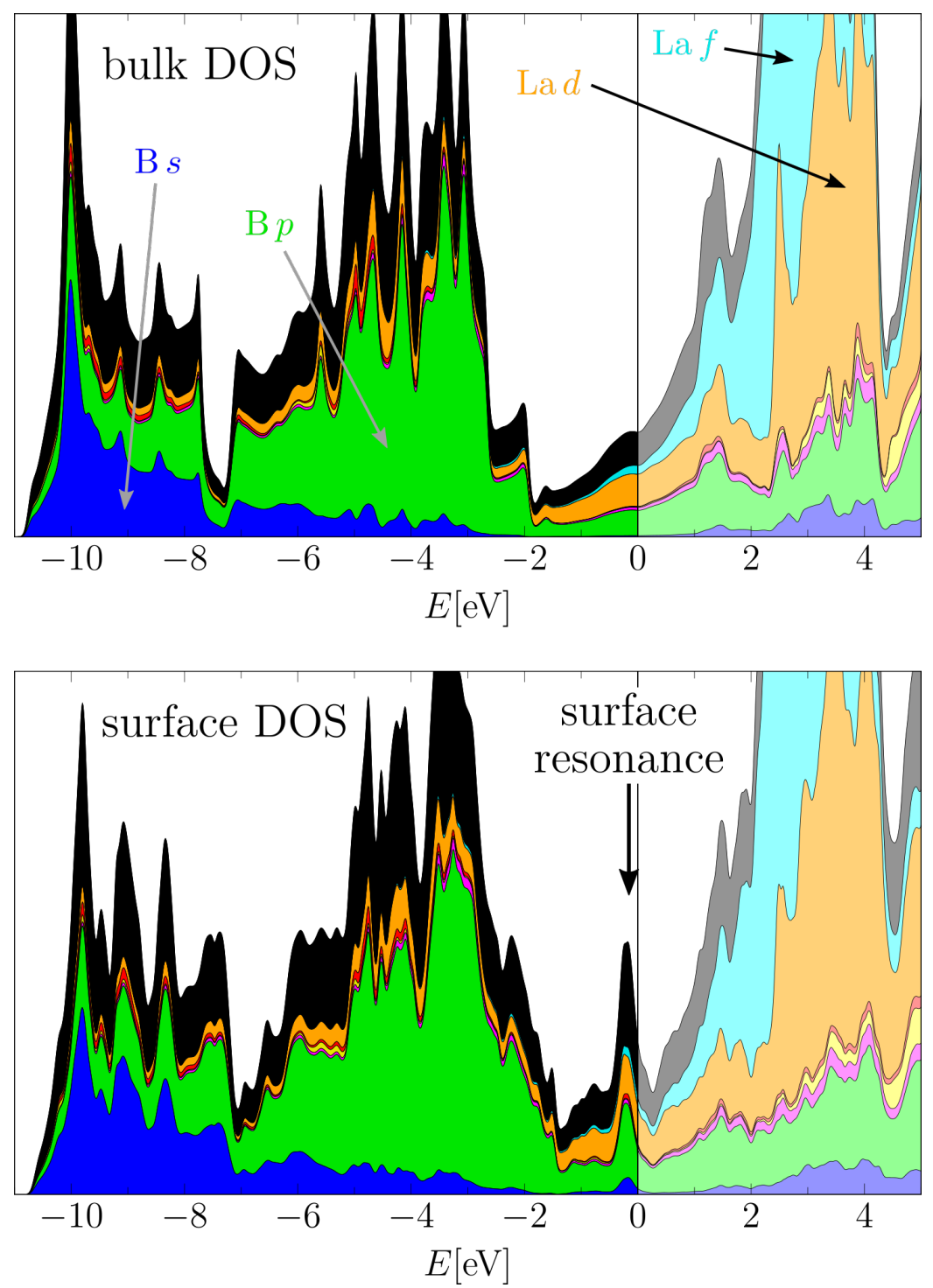

Figure 6.9: Angular momentum projected DOS of a $\mathrm{LaB}_{6}$ bulk simulation (top) and of $\mathrm{a} \mathrm{LaB}_{6}$ surface simulation (bottom). The Fermi energy is set to zero. Projected contributions are stacked. Color code: Total DOS (not stacked, black), B $s$ (blue), B $p$ (green), B $d$ (magenta), La $s$ (yellow), La $p$ (red), La $d$ (orange), La $f$ (cyan). Figure originally published in [157]. Reprinted with permission from the American Physical Society. (C) (2019) American Physical Society. 

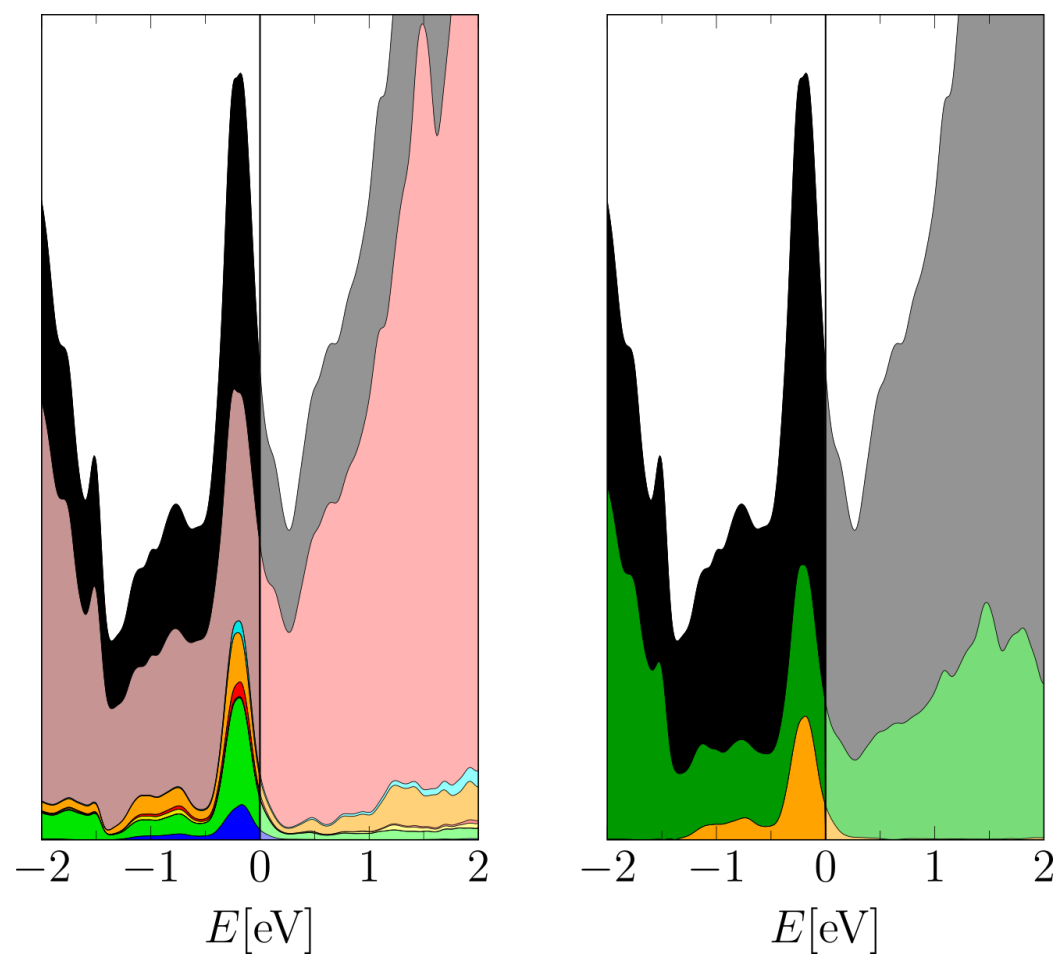

Figure 6.10: Projections of the DOS onto atomic and molecular orbitals within the slab. DOS data in arbitrary units. Individual contributions are stacked. Left: DOS projected onto shells of the outermost La ions and $\mathrm{B}_{6}$ octahedra. Color code: Total DOS (not stacked, black), B $s$ (blue), B $p$ (green), B $d$ (magenta), La $s$ (yellow), La $p$ (red), La $d$ (orange), La $f$ (cyan). The contribution of the bulk is added on top (reddish brown). Right: projection of the DOS onto boron orbitals. A large contributions of the boron dangling bonds (orange) is found at the peak at $-0.2 \mathrm{eV}$. The projection onto all other boron orbitals of the slab (green) is shown for comparison. Total DOS (black). Figure originally published in [157]. Reprinted with permission from the American Physical Society. (C) (2019) American Physical Society. 


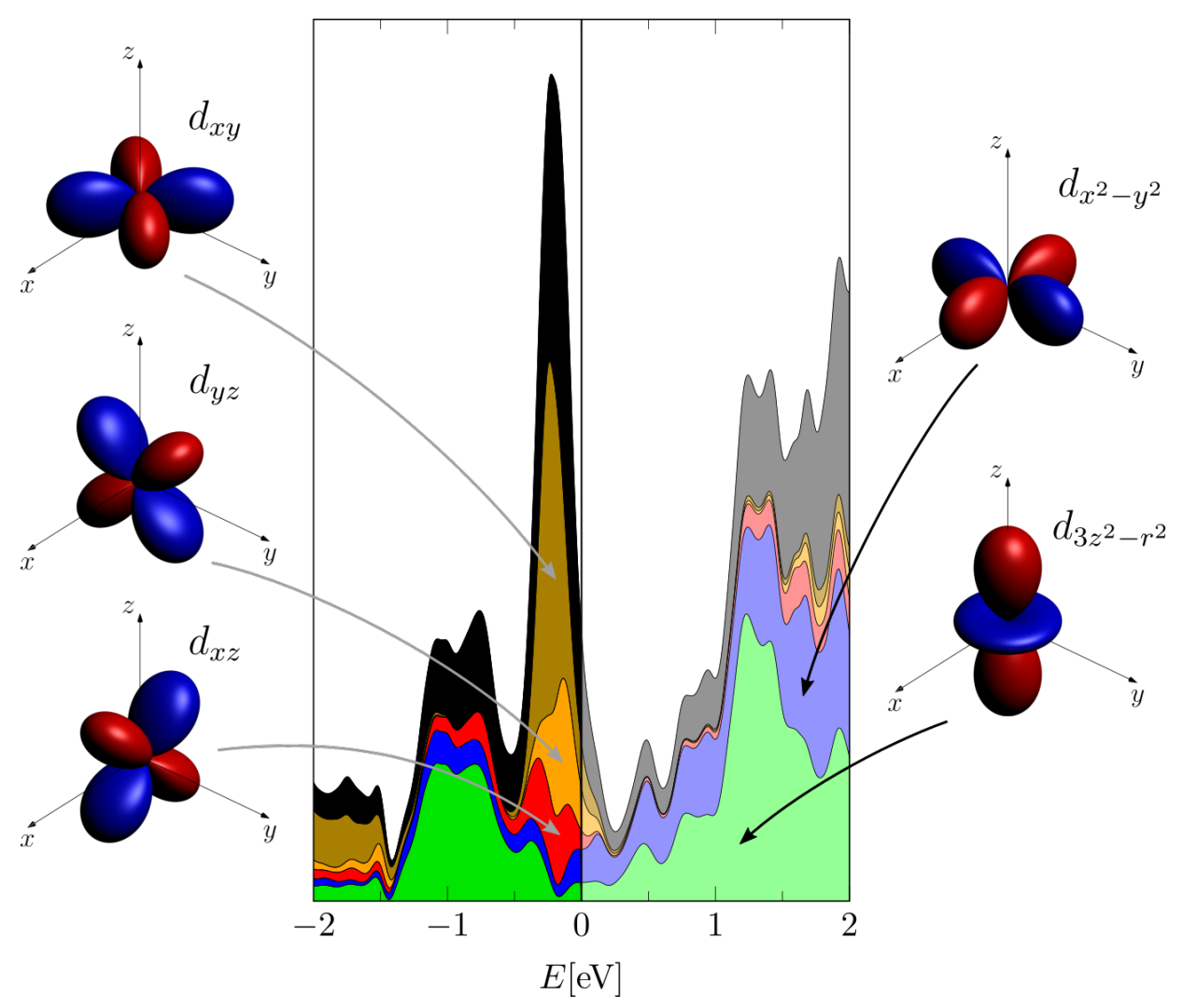

Figure 6.11: Projection of the DOS onto the individual $d$ orbitals of the lanthanum surface chains. Color code: $d_{3 z^{2}-r^{2}}$ (green), $d_{x^{2}-y^{2}}$ (blue), $d_{x z}$ (red), $d_{y z}$ (orange), $d_{x y}$ (dark gold), total DOS of the La surface ions (black). Figure originally published in [157]. Reprinted with permission from the American Physical Society. (c) (2019) American Physical Society. 
the bulk DOS. A projection of the DOS onto the electron shells of the slab's outermost La atoms and $\mathrm{B}_{6}$ octahedra (fig. 6.10, left plot) confirms that the feature is dominated by orbitals of the surface atoms. Moreover, the largest contribution originates from the boron $s p$-hybrid orbitals at the surface level that are perpendicularly sticking out of the surface and which are lacking a bonding partner due to the vacuum. This is revealed by a projection of the DOS onto these orbitals, henceforth referred to as boron dangling bonds. The respective projection of the DOS is depicted in fig. 6.10, right plot.

Another notable portion of the surface resonance at $E_{\mathrm{F}}-0.2 \mathrm{eV}$ comes from the La $d$ orbitals of the surface chains. Figure 6.11, shows the projection of the DOS onto the individual $d$ orbitals of the La ions in the surface chains. Evidently, the largest portion to the peak is contributed by the $d_{x y}$ orbitals. The lobes of the $d_{x y}$ orbital point diagonally between the crystal axes and bind with the boron dangling bonds. In the energy regions below and above the surface resonance a dominant contribution of the $d_{3 z^{2}-r^{2}}$ orbitals is found among the lanthanum $d$ orbitals. While the weights of the boron dangling bonds and the lanthanum $d$ orbitals are of equal size between $E_{\mathrm{F}}-1.29 \mathrm{eV}$ and $E_{\mathrm{F}}-0.53 \mathrm{eV}$ (see fig. 6.10, left plot), above $E_{\mathrm{F}}+0.28 \mathrm{eV}$ the boron dangling bonds vanish from the DOS and the lanthanum $d$ orbitals dominate the surface DOS.

\subsubsection{Electronic states above the surface}

According to the theory of Tersoff and Hamann with the approximation of a constant DOS of the scanning tunneling microscope (STM) tip, the tunneling current is proportional to the integrated local density of states (LDOS) of the sample at the tip apex, eq. (3.253). The spatially and energetically resolved LDOS can be extracted from the DFT data by computing the densities that originate from individual Kohn-Sham states. The contributions of states within a specified energy range are summed up to obtain the integrated LDOS for the chosen energy window. A two-dimensional slice of the integrated LDOS data above and parallel to the sample surface then produces a simulated STM topography image.

Instead of computing the integrated LDOS for the energy range $\left[E_{\mathrm{F}}+e V, E_{\mathrm{F}}\right]$ at $V<0$ and $\left[E_{\mathrm{F}}, E_{\mathrm{F}}+e V\right]$ at $V>0$, as suggested by eq. (3.253), we integrated the LDOS over energy windows that were selected based on feature boundaries within the DOS. On the one hand, choosing energy ranges according to the features in the DOS makes it easier to identify their individual contributions to the topography. On the other hand, eq. (3.253) is only an approximate relation between the tunneling current derivative and the LDOS. In fact, STM measurements are not equally sensitive across the full energy interval $\left[E_{\mathrm{F}}+\right.$ $\left.e V, E_{\mathrm{F}}\right]$ at $V<0$ and $\left[E_{\mathrm{F}}, E_{\mathrm{F}}+e V\right]$ at $V>0$, respectively, but react more strongly to features at higher energies because of the energy dependence of the tunneling exponential.

In figs. 6.12 and 6.13 we show simulated images of STM topographies for distances of $2 \AA$ and $4 \AA$ from the surface. For each distance, we include a topography image for the surface feature from $E_{\mathrm{F}}-0.53 \mathrm{eV}$ to $E_{\mathrm{F}}+0.28 \mathrm{eV}$ (center) and for the energy intervals from $E_{\mathrm{F}}-1.29 \mathrm{eV}$ to $E_{\mathrm{F}}-0.53 \mathrm{eV}$ (left) and 

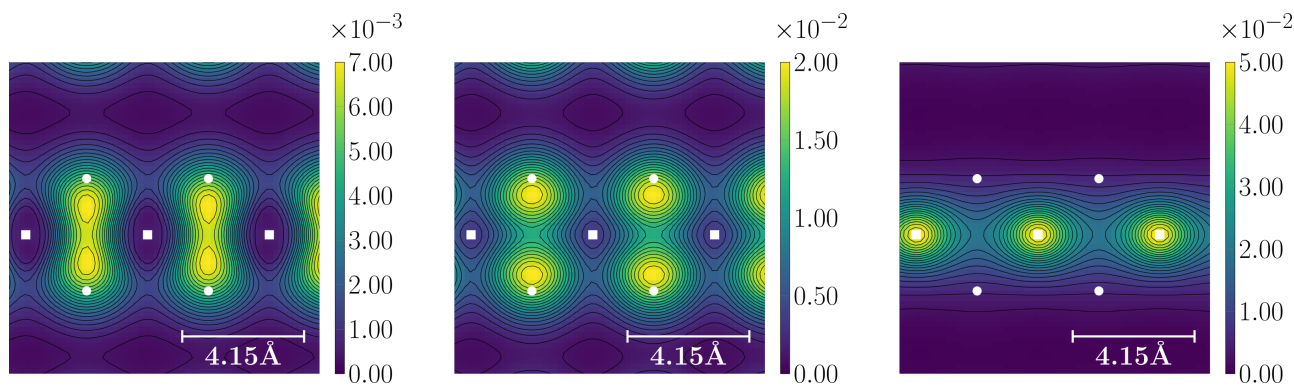

Figure 6.12: Topographic images of the electron density, integrated over three selected energy ranges at $2 \AA$ above the surface slab. Left: $-1.29 \mathrm{eV}$ to $-0.53 \mathrm{eV}$, center: $-0.53 \mathrm{eV}$ to $0.28 \mathrm{eV}$, right: $0.28 \mathrm{eV}$ to $1.64 \mathrm{eV}$. White squares mark the positions of the lanthanum atoms. Positions of boron octahedra are indicated by white circles. LDOS data in arbitrary units.
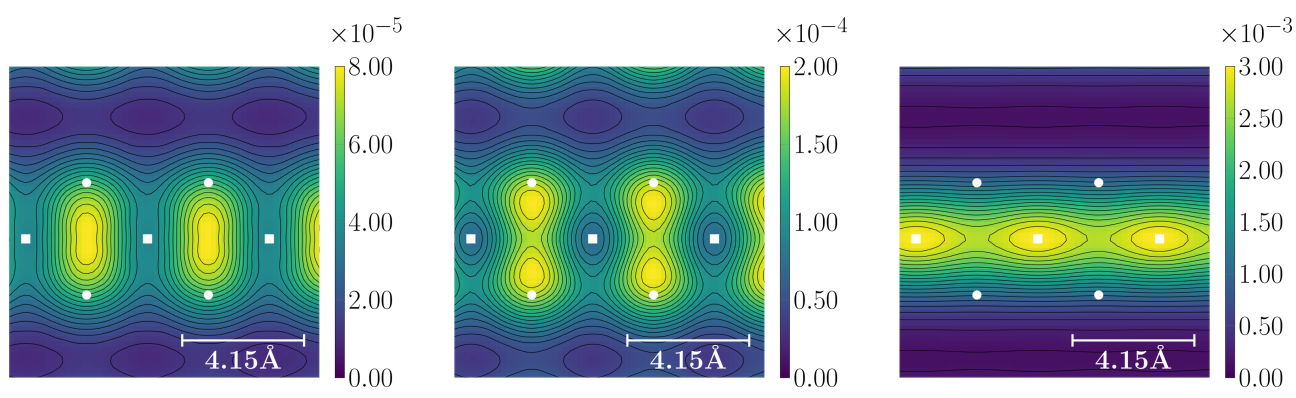

Figure 6.13: Topographic images of the electron density, integrated over three selected energy ranges at $4 \AA$ above the surface slab. Left: $-1.29 \mathrm{eV}$ to $-0.53 \mathrm{eV}$, center: $-0.53 \mathrm{eV}$ to $0.28 \mathrm{eV}$, right: $0.28 \mathrm{eV}$ to $1.64 \mathrm{eV}$. White squares mark the positions of the lanthanum atoms. Positions of boron octahedra are indicated by white circles. LDOS data in arbitrary units. Figure originally published in [157]. Reprinted with permission from the American Physical Society. (C) (2019) American Physical Society. 
from $E_{\mathrm{F}}+0.28 \mathrm{eV}$ to $E_{\mathrm{F}}+1.64 \mathrm{eV}$ (right), which are the energy ranges below and above the surface resonance, respectively. In the energy ranges below $E_{\mathrm{F}}+0.28 \mathrm{eV}$ an enhanced LDOS is located above the boron atoms, where the largest density values above the boron atoms are found in the energy range of the surface resonance from $E_{\mathrm{F}}-0.53 \mathrm{eV}$ to $E_{\mathrm{F}}+0.28 \mathrm{eV}$. Moving further away from the surface, the maxima of the integrated LDOS tend to move to the center position in between the boron atoms which are located at opposite sides of the chain structure. In both energy ranges below $E_{\mathrm{F}}+0.28 \mathrm{eV}$ the lanthanum ions are not visible. The situation changes in the energy window above $E_{\mathrm{F}}+0.28 \mathrm{eV}$, where the maxima of the integrated LDOS are located above the lanthanum ions. In this energy range, when increasing the distance to the sample surface, the maxima of the integrated LDOS are broadened and the topography receives a more chainlike structure along the underlying lanthanum chains.

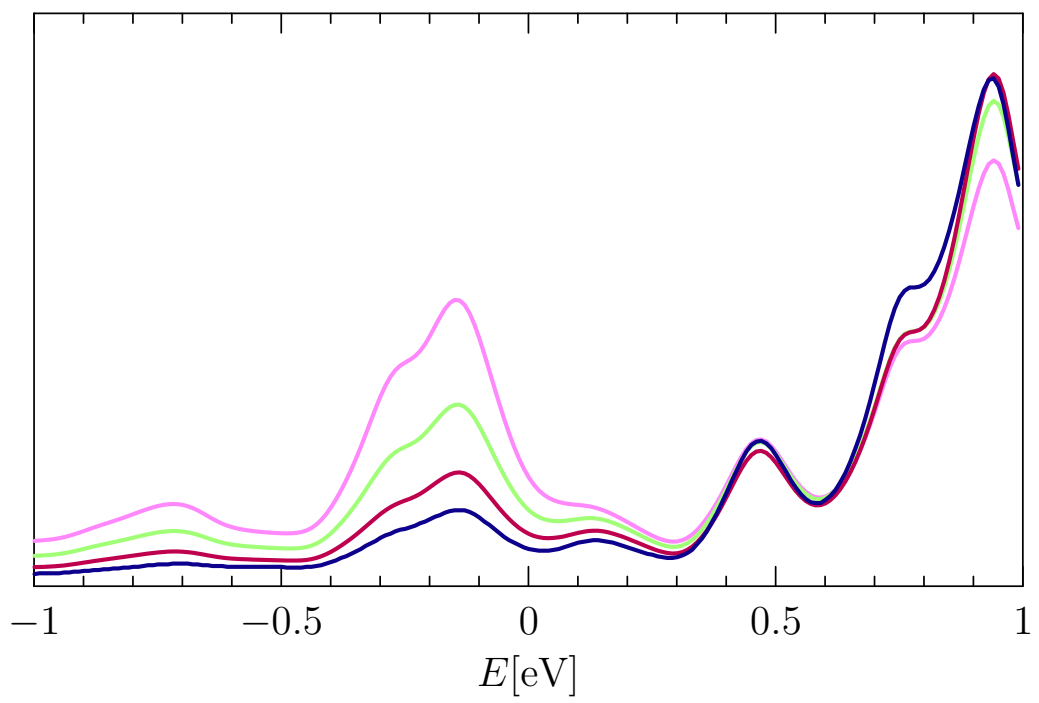

Figure 6.14: DOS projected onto layers of empty atoms parallel to the surface in arbitrary units. The layer distance from the surface is colorcoded: $4 \AA$ (pink), $5 \AA$ (green, rescaled $\times 6$ ), $6 \AA$ (red, rescaled $\times 34), 7 \AA$ (blue, rescaled $\times 200$ ). Figure originally published in [157]. Reprinted with permission from the American Physical Society. (C) (2019) American Physical Society.

For a comparison with experimental STS data we computed the spatially integrated LDOS above the surface. For this purpose, additional empty atoms were introduced in the vacuum region of the slab unit cell. These empty atoms are auxiliary entities, which provide additional orbitals in the vacuum region while leaving the physics of the system unchanged. The LDOS at the position of an empty atom is then evaluated by projecting the DOS onto the orbitals of the empty atom. Instead of computing the LDOS for several empty atoms separately, we calculated a spatially integrated LDOS by summing over multiple empty atoms that were placed in a plane parallel to the surface. We chose this approach of a plane-averaged theoretical LDOS because features coming from specific atoms will be spatially broadened at a distance of several $\AA$ from the surface. Additionally, eq. (3.254), which allows the interpretation 
of the experimental $\mathrm{d} I / \mathrm{d} V$ curves as the experimental LDOS, is a rather crude approximation. With these restrictions, a per-empty-atom-resolved LDOS is of minor additional value.

The plane-averaged LDOS is depicted in fig. 6.14 for multiple distances from the surface. Similar to the surface resonance in the DOS, the planeaveraged LDOS shows a feature at $-0.2 \mathrm{eV}$ below the Fermi energy, which can be attributed to the boron dangling bonds. Above the Fermi level a small peak occurs at $0.47 \mathrm{eV}$, which is followed by a dip and a steep rise beyond. This shape of the LDOS matches the shape of the DOS projected onto the $d_{3 z^{2}-r^{2}}$ surface states, which equally exhibits a peak at $0.47 \mathrm{eV}$ and an increasing DOS beyond. Note that, according to tunneling theory (cf. section 3.13.1), the wave functions extend further into the vacuum the higher their energy is. This is reflected in the LDOS values and has to be taken into account when comparing with the projected DOS. Because the slab wave functions decay exponentially in the vacuum region, accurate numerical data can only be obtained up to a distance of $\sim 7 \AA$ from the surface.

\subsection{Discussion}

Let us start the discussion of the results by combining the information gathered from the projected DOS, the simulated STM plots, and the LDOS curves. The appearance of the simulated topography images can be explained as follows. For energies smaller than $E_{\mathrm{F}}+0.28 \mathrm{eV}$ the boron dangling bonds dominate the surface DOS. As they point into the vacuum this leads to an enhanced density above the topmost boron positions, which is observed in the simulated topographies in the respective energy ranges. This is especially evident in the energy range $\left[E_{\mathrm{F}}-0.53 \mathrm{eV}, E_{\mathrm{F}}+0.28 \mathrm{eV}\right]$ that contains the surface resonance. Further, the lanthanum ions are not visible in the topographies at energies below $E_{\mathrm{F}}+0.28 \mathrm{eV}$ because the lanthanum $d$ orbitals are more localized than the boron $s p$-hybrid dangling bonds. Additionally, in the $\left[E_{\mathrm{F}}-0.53 \mathrm{eV}, E_{\mathrm{F}}+\right.$ $0.28 \mathrm{eV}$ ] energy range of the surface resonance the predominant $d$ orbitals cannot contribute to the density directly above the lanthanum ions because of their nodal shapes (cf. fig. 6.11). In the DOS of the surface states, the contribution of the boron dangling bonds vanishes at energies above $E_{\mathrm{F}}+$ $0.28 \mathrm{eV}$ and the lanthanum $d$ states provide the largest share. Among the lanthanum $d$ states, the $d_{3 z^{2}-r^{2}}$ orbital dominates in the energy range from $E_{\mathrm{F}}+0.28 \mathrm{eV}$ to $E_{\mathrm{F}}+1.64 \mathrm{eV}$. Since the $d_{3 z^{2}-r^{2}}$ orbital points perpendicularly out of the surface this leads to an enhanced integrated LDOS above the lanthanum ions and to the chainlike structure in the topography images. Note that the lanthanum $d$ states from the energy range $\left[E_{\mathrm{F}}+0.28 \mathrm{eV}, E_{\mathrm{F}}+1.64 \mathrm{eV}\right]$ extend further into the vacuum than the lanthanum $d$ states from the energy range $\left[E_{\mathrm{F}}-1.29 \mathrm{eV}, E_{\mathrm{F}}-0.53 \mathrm{eV}\right]$ because the wave function has an exponential dependence on the energy in the vacuum region.

The exponential dependence of the wave functions is especially clear in the LDOS curves of fig. 6.14. Above $E_{\mathrm{F}}+0.28 \mathrm{eV}$ the main contribution to the LDOS comes from the $d_{3 z^{2}-r^{2}}$ orbital, which is evident from a comparison of 
the projected DOS in fig. 6.11 and the LDOS in fig. 6.14. The LDOS rises more steeply at larger energies than the projected DOS of the $d_{3 z^{2}-r^{2}}$ orbital because it is affected by the tunneling exponential. Below the Fermi level the spatial extension of the $d_{3 z^{2}-r^{2}}$ orbital is rather small because of the comparatively small tunneling exponential. This is a reason for the small density above the lanthanum ions in the simulated STM images of the lower lying energy ranges. Moreover, at these energies the boron dangling bonds are dominant in the LDOS and the surface resonance at $E_{\mathrm{F}}-0.2 \mathrm{eV}$ can be clearly identified.

The LDOS data also clearly shows that the surface resonance decays faster at increased distance than the features produced by the lanthanum orbitals above the Fermi level. This can be rationalized by a faster decay of the tunneling exponential for an increased distance from the surface at smaller energies. The further extent of the lanthanum $d_{3 z^{2}-r^{2}}$ states into the vacuum in comparison to the boron dangling bonds is also seen in the simulated STM images by the larger density values of the $\left[E_{\mathrm{F}}+0.28 \mathrm{eV}, E_{\mathrm{F}}+1.64 \mathrm{eV}\right]$ energy window in comparison with the $\left[E_{\mathrm{F}}-1.29 \mathrm{eV}, E_{\mathrm{F}}-0.53 \mathrm{eV}\right]$ and $\left[E_{\mathrm{F}}-0.53 \mathrm{eV}, E_{\mathrm{F}}+0.28 \mathrm{eV}\right]$ ranges.

In experimental STM topographies chainlike and labyrinthlike $(2 \times 1)$ reconstructions were observed in measurements with a positive sample bias voltage, such that the preferential tunneling direction is from the tip to the sample and sample states above the Fermi level are addressed. Our simulated STM images from the energy range $\left[E_{\mathrm{F}}+0.28 \mathrm{eV}, E_{\mathrm{F}}+1.64 \mathrm{eV}\right]$ match the experimental observations and exhibit a chainlike arrangement of density maxima along the lanthanum chains at a distance of several $\AA$ above the lanthanum ions. The dominant contribution to the increased density above the lanthanum ions is shown to come from the lanthanum $d_{3 z^{2}-r^{2}}$ orbitals.

In the energy ranges $\left[E_{\mathrm{F}}-1.29 \mathrm{eV}, E_{\mathrm{F}}-0.53 \mathrm{eV}\right]$ and $\left[E_{\mathrm{F}}-0.53 \mathrm{eV}, E_{\mathrm{F}}+\right.$ $0.28 \mathrm{eV}]$ the LDOS maxima appear above the boron octahedra. It is clear from the projected DOS that these maxima originate from the boron dangling bonds that are shown to lie within these energy windows. Unfortunately, unstable tunneling conditions at negative bias voltages did not allow to record experimental CCTs at these energies for a direct comparison with our simulated STM topographies. However, experimental measurements at negative bias voltage were successful with STS. The shapes of the experimental $\mathrm{d} I / \mathrm{d} V$ curves and our theoretical LDOS are in good agreement. Note, that the feature at $-0.6 \mathrm{eV}$ in the experimental $\mathrm{d} I / \mathrm{d} V$ curves in fig. 6.6(b) and the feature at $0 \mathrm{eV}$ to $0.1 \mathrm{eV}$ in fig. 6.6(d) would match the peaks at $-0.2 \mathrm{eV}$ and $0.45 \mathrm{eV}$ in our theoretical LDOS, if the Fermi level in the theoretical data was shifted upwards by $0.4 \mathrm{eV}$. Such a shift of the Fermi level can occur when the atomic ratio La/B in the crystal is slightly larger than $1 / 6$. In that case a surplus of lanthanum leads to additional electrons in the material and thus to a higher Fermi level.

It is worth mentioning that the position of the surface resonance is resistant to defects. This conclusion arises from the fact that in the spatially resolved STS data the surface resonance is observed at $-0.6 \mathrm{~V}$ bias voltage, regardless of morphological details of the surface. Furthermore, the $\mathrm{d} I / \mathrm{d} V$ data agrees well with the LDOS data of our theoretical model, which has a perfectly ordered, defect-free surface morphology. Additionally, we emphasize that the position of the surface resonance is unaltered when changing the DFT functional from 
PBE0r to PBE. This is to be expected since the surface resonance is mostly coming from boron states, which are barely affected by the exchange mixing within the PBE0r hybrid functional.

Following the above discussion, we find a good agreement of the experimental STM and STS data with our theoretical results of DFT simulations of a $\mathrm{LaB}_{6}(001)$ surface slab with chains of lanthanum ions at the surface. Hence, we conclude that the chainlike and labyrinthlike structures in the STM CCTs of atomically flat surface areas at positive sample bias voltage can indeed be regarded as chainlike and labyrinthlike arrangements of La ions at the surface, respectively. For two such areas that are separated by a step, the step height has to be an integer multiple of the lattice constant. This is in agreement with observed steps. Furthermore, we suggest that the area in the lower left corner of figure fig. 6.5 is boron terminated since it is separated from the lanthanumterminated surface area by a step of a fraction of the lattice constant.

We confirm the realization of a $(2 \times 1)$ surface reconstruction on the $\mathrm{LaB}_{6}$ (001) cleavage plane. This is backed by the observation of $(2 \times 1)$ and $(1 \times 2)$ spot patterns in LEED measurements, where a $(1 \times 2)$ pattern corresponds to a $(2 \times 1)$ pattern of a surface area with lanthanum chains that is rotated by $90^{\circ}$. The dominant $(1 \times 1)$ spots can be ascribed to the penetration of the electrons into the first few bulk layers beneath the surface.

In previous experimental studies of the $\mathrm{LaB}_{6}(001)$ surface with polished and heat-treated samples, surfaces states have been observed at $-2 \mathrm{eV}$ below the Fermi level [50]. These surface states have been attributed to the boron dangling bonds and have been suggested to bind with orbitals of terminal lanthanum ions. Since polished and heat-treated samples showed an unreconstructed $(1 \times 1)$ surface, additional lanthanum states exist at the surface for bonding with the boron dangling bonds in comparison with a lanthanum-terminated $(2 \times 1)$ surface reconstruction. Hence, for an unreconstructed surface, the boron dangling bonds are more tightly bound to the surface and will have lower energies. This explains the different energies of the boron dangling bonds of $-2 \mathrm{eV}$ for an unreconstructed $(1 \times 1)$ surface and of $-0.6 \mathrm{eV}$ for a $(2 \times 1)$ surface reconstruction. 


\section{Summary and perspective}

The research in the context of this thesis had two main aims:

1. Assess whether the PBE0r local hybrid functional is suitable for density functional theory (DFT) simulations of the rare-earth hexaborides as representatives of strongly correlated, metallic electron systems.

2. Clarify and explain the atomic and electronic structure of the lanthanum hexaboride (001) cleavage plane.

Concerning the first goal, we performed DFT bulk simulations of the light rareearth hexaborides $\mathrm{LaB}_{6}, \mathrm{CeB}_{6}, \mathrm{PrB}_{6}$, and $\mathrm{NdB}_{6}$, for which we employed the PBE0r local hybrid functional. The PBE0r functional is based on the PerdewBurke-Ernzerhof (PBE) functional, a functional within the generalized gradient approximation, and mixes the PBE exchange contribution with a portion of local Hartree-Fock exchange. The exchange mixing within the PBE0r functional improves upon the incomplete compensation of the self-interaction that plagues conventional functionals within the local spin density approximation and the generalized gradient approximation. A set of parameters is used to fix the exchange mixing strength on a per-atom basis.

With the PBE0r hybrid functional the densities of states from our simulations of $\mathrm{CeB}_{6}, \mathrm{PrB}_{6}$, and $\mathrm{NdB}_{6}$ show an energy separation of the occupied and unoccupied $4 f$ states, which is in contrast to simulations with the PBE functional. The energy separation is an expected consequence of the improved self-interaction compensation. Its width can be tuned by adjusting the exchange mixing strength.

In the case of $\mathrm{CeB}_{6}$ a single occupied $4 f$ state is split from the unoccupied $4 f$ orbitals. This state can be shifted to the binding energy at which it is found in experimental photoemission spectra by choosing a suitable value for the exchange mixing strength within the hybrid functional.

For $\mathrm{PrB}_{6}$, the DFT computational scheme is found to converge into three different types of final states of the set of Kohn-Sham states and their occupancies. One of these states was nearly spin-degenerate and identified to give a local, but not global minimum of the total energy. The other two states, termed TYPE II and III, were found at the same minimum total energy, which indicates a degenerate ground state for certain exchange mixing strengths. Also for $\mathrm{PrB}_{6}$ the occupied $4 f$ levels can be shifted to the energy of the $4 f^{2} \rightarrow 4 f^{1}$ ionization peak in experimental photoemission data. At the respective exchange mixing strength only the TYPE II ground state is found. This ground state possesses two occupied $4 f$ levels, which are energetically well separated from the unoccupied levels. 
For $\mathrm{NdB}_{6}$, we find a single ground state with a fractional occupancy of the $4 f$ shell, which is similar to the TyPE III state of $\operatorname{PrB}_{6}$. In particular, the $4 f$ projected DOS has a three-peak shape. Two peaks are comprised of the fully occupied and unoccupied states, respectively. Moreover, a third peak coming from a fractionally occupied $4 f$ level is pinned to the Fermi level. From the simulation results of $\mathrm{NdB}_{6}$ it is clear that the DOS cannot be brought into agreement with spectra from photoemission experiments by tuning the exchange mixing strength within the PBE0r functional. The occupied $4 f$ states are located at least $3.5 \mathrm{eV}$ above the experimentally determined binding energy.

The unclear origin of the three-peak shape of the $4 f$-projected DOSs in $\mathrm{PrB}_{6}$ and $\mathrm{NdB}_{6}$ and, in particular, the occurrence of the fractionally occupied $4 f$ level at the Fermi level motivated further investigations on the basis of a simple model system. Based on the results of our model study we find the following conclusion. The $4 f$-projected DOS is to be understood as the spectral function of an effective non-interacting system. This is owed to the Kohn-Sham formalism of DFT, in which an interacting material is described in terms of an effective system of non-interacting Kohn-Sham states, in conjunction with the exchange mixing of the PBE0r functional. However, for a fractional occupancy of the $4 f$ shell, this spectral function of the effective non-interacting system does not represent the $4 f$ spectral function of the real material and hence cannot be taken as a prediction for experimental photoemission spectra. This finding is especially important for simulations of materials in which noninteracting metallic states and interacting localized orbitals are coupled via a small hybridization. In such systems a fractional occupancy of the localized orbitals can occur. On the other hand, our model results do not suggest that this problem occurs in the case of an integer occupancy of the localized orbitals. An example for such a material is $\mathrm{CeB}_{6}$. Moreover, one can expect that the DOS of an insulator is not affected by this problem.

While it is clear that the $4 f$-projected DOS of $\mathrm{NdB}_{6}$ does not represent the photoemission spectrum, our model calculation allows to propose a prescription for the reconstruction of the spectral function of the interacting material. Employing this "rule of thumb" we recover a $4 f$-projected spectral function for $\mathrm{NdB}_{6}$, which is in agreement with available photoemission data. In particular, the reconstructed spectral function exhibits an excitation peak at the energy of the $4 f^{3} \rightarrow 4 f^{2}$ ionization peak in experimental photoemission spectra. Further, the reconstructed spectral function also shows an excitation peak at the Fermi level. Interestingly, an unusual renormalization has been observed in photoemission spectra of $\mathrm{PrB}_{6}$ and $\mathrm{NdB}_{6}$ in the vicinity of the Fermi level [30]. However, from our present model a link between the excitation peak in the spectral function of the model and the enhanced spectral features found in the photoemission data in the vicinity of the Fermi level is not evident.

A shortcoming of the model is the rather crude treatment of the hybridization between the localized and metallic states. In this regard, an improved version of our simple model would be a multi-orbital Anderson impurity model (AIM), which can be solved numerically with quantum Monte Carlo (QMC) algorithms. As a follow-up on the present study, a continuous-time QMC solver [154] in the hybridization expansion formulation can be utilized to compute an improved 
$4 f$-projected spectral function. Parameters for the AIM can be obtained from our present model and the DOS of the metallic states of the hexaboride. Modern versions of the QMC solver even allow for complex hopping matrices in the impurity Hamiltonian, which opens the possibility to include the spin-orbit interaction [155]. We expect that a study of the AIM by means of QMC will allow to assess the quality of our proposed "rule of thumb" for the reconstruction of the $4 f$-projected DOS of the hexaborides. Moreover, it certainly seems interesting to apply the computational scheme to further materials with strongly localized electronic states in order to test our "rule of thumb" for general validity. Because of the full many-particle treatment of the AIM in the QMC algorithm, a QMC study might also shed some light on the origin of the unusual spectral renormalization near the Fermi level in the photoemission spectra of $\mathrm{PrB}_{6}$ and $\mathrm{NdB}_{6}$.

One of the main successes of our model study is that the effect of the local exchange term onto the DOSs of the rare-earth hexaborides is now well understood. Moreover, the study also shows that the effect of the double counting term, which in the PBE0r hybrid functional is formulated on the DFT level, can be rationalized with the help of the double counting models of the LDA $+U$ method.

As the second pillar of this thesis we conducted a joint study of the $\mathrm{LaB}_{6}$ (001) cleavage plane, in which the data of our experimental collaborators go hand in hand with the results of our DFT simulations. Our simulation data of a $\mathrm{LaB}_{6}$ slab that is terminated by chains of lanthanum ions on top of a $\mathrm{B}_{6}$ layer are in agreement with experimental data on the $\mathrm{LaB}_{6}$ cleavage plane, which have been obtained from scanning tunneling microscopy, scanning tunneling spectroscopy, and low-energy electron diffraction measurements. In particular, the results of our study show that the chainlike and labyrinthlike structures within the STM constant current topographies of atomically flat areas of the $\mathrm{LaB}_{6}(001)$ cleavage plane at positive sample bias voltage correspond to the underlying arrangement of lanthanum ions on top of a $\mathrm{B}_{6}$ layer at the sample surface. The enhanced local density of states above the lanthanum positions is traced back to the La $d_{3 z^{2}-r^{2}}$ orbitals, which dominate the energy range above the Fermi level. A major finding of our study is a surface resonance in the DOS at $0.2 \mathrm{eV}$ below the Fermi level. This surface resonance originates from unpaired $s p$-hybrid states of the boron framework at the surface level. These "boron dangling bonds" lead to an enhanced local density of states above the boron positions. An additional contribution to the surface resonance comes from the lanthanum $d_{x y}$ orbitals, which bind into the boron dangling bonds.

Our study of the $\mathrm{LaB}_{6}$ cleavage plane shows that the electronic structure of the $\mathrm{LaB}_{6}$ surface differs significantly from the electronic structure of the $\mathrm{LaB}_{6}$ bulk. Moreover, it is clear that the surface morphology of the $\mathrm{LaB}_{6}$ cleavage plane is different from that of polished and heat-treated $\mathrm{LaB}_{6}$ surface samples that have been investigated in earlier studies.

We expect the present study of the $\mathrm{LaB}_{6}$ surface to help towards a general understanding of the surface physics of the rare-earth hexaborides. All rareearth hexaborides have the same crystal structure with only small variations in the lattice constants. We can hence assume a similar electronic structure 
coming from the boron states, if a similar surface morphology is found. Our surface study of $\mathrm{LaB}_{6}$, which does not possess occupied $4 f$ levels, could help to identify spectral features originating from $4 f$ levels in future surface studies of rare-earth hexaborides with lanthanide constituents that possess a partially occupied $4 f$ shell.

In regard to surface simulations of the rare-earth hexaborides with a partially occupied $4 f$ shell by means of DFT with the PBE0r hybrid functional, further work is required before reliable theoretical spectral functions can be obtained for comparison with experimental spectra, e. g. from scanning tunneling spectroscopy. Here, the correct theoretical description of the bulk spectra should be regarded as a necessary precursor. 


\section{Appendices}





\section{A Supplemental material on the deriva- tion of the Hartree-Fock approxima- tion}

In the derivation of the Hartree-Fock approximation in section 3.7 we consider an interacting fermionic system, which is coupled to a bath. The interacting system can exchange energy and particles with the bath. An auxiliary noninteracting system with an effective potential that approximately captures the interaction effects is introduced as an approximate model to the interacting system. In the course of the derivation we need to compute the expectation value $\langle\hat{H}\rangle_{\mathrm{HF}}$ of the interacting Hamiltonian with respect to the density operator $\hat{\Gamma}_{\mathrm{HF}}$, see eq. (3.61), of the effective non-interacting Hamiltonian. The evaluation of $\langle\hat{H}\rangle_{\mathrm{HF}}$ requires the evaluation of the expectation value $\langle\hat{W}\rangle_{\mathrm{HF}}$ of the interaction operator $\hat{W}$.

Here, we show that the expectation value of the interaction operator $\hat{W}$ with respect to the effective non-interacting system, indicated by a subscript $\mathrm{HF}$, can be expressed in the following way:

$$
\begin{aligned}
\langle\hat{W}\rangle_{\mathrm{HF}} & =\frac{1}{2} \sum_{a b c d} U_{a b c d}\left\langle\hat{c}_{a}^{\dagger} \hat{c}_{b}^{\dagger} \hat{c}_{d} \hat{c}_{c}\right\rangle_{\mathrm{HF}} \\
& =\frac{1}{2} \sum_{a b c d} U_{a b c d}\left(\left\langle\hat{c}_{a}^{\dagger} \hat{c}_{c}\right\rangle_{\mathrm{HF}}\left\langle\hat{c}_{b}^{\dagger} \hat{c}_{d}\right\rangle_{\mathrm{HF}}-\left\langle\hat{c}_{a}^{\dagger} \hat{c}_{d}\right\rangle_{\mathrm{HF}}\left\langle\hat{c}_{b}^{\dagger} \hat{c}_{c}\right\rangle_{\mathrm{HF}}\right) \\
& =\frac{1}{2} \sum_{a b c d}\left(U_{d a c b}-U_{d a b c}\right)\left\langle\hat{c}_{d}^{\dagger} \hat{c}_{c}\right\rangle_{\mathrm{HF}}\left\langle\hat{c}_{a}^{\dagger} \hat{c}_{b}\right\rangle_{\mathrm{HF}}
\end{aligned}
$$

To prove the above equations we first use Wick's theorem to show that

$$
\left\langle\Phi\left|\hat{c}_{a}^{\dagger} \hat{c}_{b}^{\dagger} \hat{c}_{c} \hat{c}_{d}\right| \Phi\right\rangle=\rho_{d a} \rho_{c b}-\rho_{c a} \rho_{d b}
$$

The expectation value of the operator sequence within the interaction operator $\hat{W}$ with respect to a single Slater determinant can be expressed in terms of elements of the one-particle density matrix $\rho$. This result is used in the second step, where we show relation (A.3). 


\section{A.1 Expectation value of the interaction Hamilto- nian with respect to a Slater determinant by Wick's theorem}

Wick's theorem [162-164] is a rule for expressing a sequence of time-ordered creation and annihilation operators in terms of a sum of so-called normalordered operator sequences. The time-ordering of a sequence of operators $\hat{A}$, $\hat{B}, \hat{C}$, and $\hat{D}$ which act a different moments in time is often expressed by the time-ordering operator $\hat{\mathcal{T}}$. We are interested in the case, where the operators $\hat{A}$, $\hat{B}, \hat{C}$, and $\hat{D}$ are either fermionic creation operators $\hat{c}^{\dagger}$ or fermionic annihilation operators $\hat{c}$. Then, an example for the time ordering of a sequence of operators is

$$
\hat{\mathcal{T}}\left(\hat{A}\left(t_{2}\right) \hat{B}\left(t_{3}\right) \hat{C}\left(t_{1}\right) \hat{D}\left(t_{2}\right)\right)=-\hat{C}\left(t_{1}\right) \hat{A}\left(t_{2}\right) \hat{D}\left(t_{2}\right) \hat{B}\left(t_{3}\right), \quad \text { where } t_{1}>t_{2}>t_{3} .
$$

The sign is chosen according to $(-1)^{N_{P}}$, where $N_{P}$ is the number of operator exchanges that need to be made to arrive at the correct time ordering. Operators with the same time argument are time-ordered in their existing order.

A sequence of creation and annihilation operators is said to be normalordered, if all creation operators occur on the left of all annihilation operators. Again, we are interested in the case for fermions,

$$
\hat{\mathcal{N}}\left(\hat{c}_{i} \hat{c}_{j}^{\dagger} \hat{c}_{k} \hat{c}_{l}^{\dagger}\right)=-\hat{c}_{j}^{\dagger} \hat{c}_{l}^{\dagger} \hat{c}_{i} \hat{c}_{k}
$$

where the sign is positive for an even permutation and negative for an odd permutation of the operators.

We also define the contraction of two operators as the difference of the time-ordered and the normal-ordered sequence of two operators,

$$
\hat{A} \hat{B}=\hat{\mathcal{T}}(\hat{A} \hat{B})-\hat{\mathcal{N}}(\hat{A} \hat{B})
$$

For fermionic creation and annihilation operators this gives the following rules:

$$
\hat{c}_{i}^{\dagger} \hat{c}_{j}^{\dagger}=0, \quad \hat{c}_{i} \hat{c}_{j}=0, \quad \hat{c}_{i}^{\dagger} \hat{c}_{j}=0, \quad \text { and } \quad \hat{c}_{i} \hat{c}_{j}^{\dagger}=\hat{c}_{i} \hat{c}_{j}^{\dagger}+\hat{c}_{j}^{\dagger} \hat{c}_{i}=\delta_{i j} .
$$

According to Wick's theorem, a sequence of time-ordered operators can be expressed as

$$
\begin{aligned}
\hat{\mathcal{T}}(\hat{A} \hat{B} \hat{C} \hat{D} \hat{E} \hat{F} \ldots)= & \hat{\mathcal{N}}(\hat{A} \hat{B} \hat{C} \hat{D} \hat{E} \hat{F} \ldots) \\
& +\underbrace{\hat{\mathcal{N}}(\hat{A} \hat{B} \hat{C} \hat{D} \hat{E} \hat{F} \ldots)+\ldots}_{\text {single contractions }}
\end{aligned}
$$


A.1 Expectation value of the interaction Hamiltonian with respect to a Slater determinant by Wick's theorem

$$
\begin{aligned}
& +\underbrace{\hat{\mathcal{N}}(\hat{A} \hat{B} \hat{C} \hat{D} \hat{E} \hat{F} \ldots)+\ldots}_{\text {double contractions }} \\
& +\ldots \quad \\
& +\underbrace{\hat{\mathcal{N}}(\hat{A} \hat{B} \hat{C} \hat{D} \hat{E} \hat{F} \hat{F} \ldots)+\ldots}_{\text {full contractions }}
\end{aligned}
$$

In the following we apply Wick's theorem to $\left\langle\Phi\left|\hat{c}_{i}^{\dagger} \hat{c}_{j}^{\dagger} \hat{c}_{k} \hat{c}_{l}\right| \Phi\right\rangle$, where $|\Phi\rangle$ is a Slater determinant. For simplicity we choose the creation and annihilation operators to be in the basis in which the one-particle density matrix associated with $|\Phi\rangle$ is diagonal. The expectation value $\left\langle\Phi\left|\hat{c}_{i}^{\dagger} \hat{c}_{j}^{\dagger} \hat{c}_{k} \hat{c}_{l}\right| \Phi\right\rangle$ is equivalent to the vacuum expectation value

$$
\left\langle\Phi\left|\hat{c}_{i}^{\dagger} \hat{c}_{j}^{\dagger} \hat{c}_{k} \hat{c}_{l}\right| \Phi\right\rangle=\left\langle 0\left|\hat{c}_{1} \ldots \hat{c}_{N} \hat{c}_{i}^{\dagger} \hat{c}_{j}^{\dagger} \hat{c}_{k} \hat{c}_{l} \hat{c}_{N}^{\dagger} \ldots \hat{c}_{1}^{\dagger}\right| 0\right\rangle .
$$

Since the operators in eq. (A.10) do not have a time-dependence, the given operator sequence is already time-ordered. Applying Wick's theorem, all contractions other than full contractions vanish because the vacuum expectation value of a normal-ordered operator sequence is zero. From the remaining full contractions all those contractions vanish where a contraction occurs with two operators with different quantum numbers. For simplicity and without loss of generality we sort the Slater determinant $|\Phi\rangle$ such that the orbitals $i$ and $j$ are the orbitals 1 and 2 of the Slater determinant. In this case, we need to consider only the following remaining contractions,

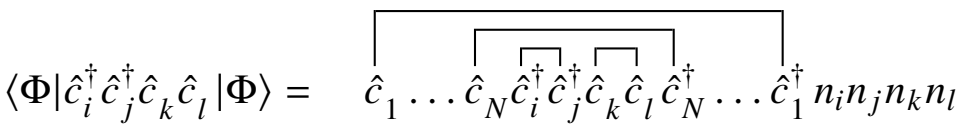

$$
\begin{aligned}
& +\hat{c}_{1} \ldots \hat{c}_{N} \hat{c}_{i}^{\dagger} \hat{c}_{j}^{\dagger} \hat{c}_{k} \hat{c}_{l} \hat{c}_{N}^{\dagger} \ldots \hat{c}_{1}^{\dagger} n_{i} n_{j} n_{k} n_{l} \\
& +\hat{c}_{1} \ldots \hat{c}_{N} \hat{c}_{i}^{\dagger} \hat{c}_{j}^{\dagger} \hat{c}_{k} \hat{c}_{l} \hat{c}_{N}^{\dagger} \ldots \hat{c}_{1}^{\dagger} n_{i} n_{j} n_{k} n_{l}
\end{aligned}
$$

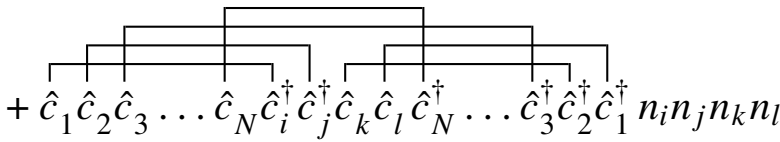

$$
\begin{aligned}
& +\hat{c}_{1} \hat{c}_{\hat{c}_{2} \hat{c}_{3} \ldots \hat{c}_{N} \hat{c}_{i}^{\dagger} \hat{c}_{j}^{\dagger} \hat{c}_{k} \hat{c}_{k} \hat{c}_{l} \hat{c}_{N}^{\dagger} \ldots \hat{c}_{3}^{\dagger} \hat{c}_{2}^{\dagger}} \hat{c}_{1}^{\dagger} n_{i} n_{j} n_{k} n_{l} \\
& +\hat{\hat{c}}_{1} \hat{c}_{2} \hat{c}_{3} \ldots \hat{c}_{N} \hat{c}_{i}^{\dagger} \hat{c}_{j}^{\dagger} \hat{c}_{k} \hat{c}_{k} \hat{c}_{l} \hat{c}_{N}^{\dagger} \ldots \hat{c}_{3}^{\dagger} \hat{c}_{2}^{\dagger} \hat{c}_{1}^{\dagger} n_{i} n_{j} n_{k} n_{l} \\
& +\hat{c}_{1} \hat{c}_{\hat{c}_{2} \hat{c}_{3} \ldots \hat{c}_{N} \hat{c}_{i}^{\dagger} \hat{c}_{j}^{\dagger} \hat{c}_{k} \hat{c}_{l} \hat{c}_{N}^{\dagger} \ldots \hat{c}_{3}^{\dagger} \hat{c}_{2}^{\dagger}} \hat{c}_{1}^{\dagger} n_{i} n_{j} n_{k} n_{l}
\end{aligned}
$$


(A.12)

Note that the product of the occupation numbers with $n \in\{0,1\}$ occurs after each contraction because the expectation value vanishes if one of the orbitals $i, j, k$, and $l$ is unoccupied. The first three full contractions are zero according to the contraction rules in eq. (A.8). Next, we untangle the remaining full contractions,

$$
\begin{aligned}
& \left\langle\Phi\left|\hat{c}_{i}^{\dagger} \hat{c}_{j}^{\dagger} \hat{c}_{k} \hat{c}_{l}\right| \Phi\right\rangle=\widehat{\hat{c}}_{1} \hat{c}_{2} \hat{c}_{i}^{\dagger} \hat{c}_{j}^{\dagger} \hat{c}_{k} \hat{c}_{l} \hat{c}_{2}^{\dagger} \hat{c}_{1}^{\dagger} \hat{c}_{3} \hat{c}_{3}^{\dagger} \ldots \hat{c}_{N} \hat{c}_{N}^{\dagger} n_{i} n_{j} n_{k} n_{l} \\
& +\hat{c}_{1} \hat{c}_{2} \hat{c}_{i}^{\dagger} \hat{c}_{j}^{\dagger} \hat{c}_{k} \hat{c}_{l} \hat{c}_{2}^{\dagger} \hat{c}_{1}^{\dagger} \hat{c}_{3} \hat{c}_{3}^{\dagger} \ldots \widehat{c}_{N} \hat{c}_{N}^{\dagger} n_{i} n_{j} n_{k} n_{l} \\
& +\widehat{c}_{1} \hat{c}_{2} \hat{c}_{i}^{\dagger} \hat{c}_{j}^{\dagger} \hat{c}_{k} \hat{c}_{l} \hat{c}_{2}^{\dagger} \hat{c}_{1}^{\dagger} \widehat{c}_{3} \hat{c}_{3}^{\dagger} \ldots \hat{c}_{N} \hat{c}_{N}^{\dagger} n_{i} n_{j} n_{k} n_{l} \\
& +\hat{c}_{1} \hat{c}_{2} \hat{c}_{i}^{\dagger} \hat{c}_{j}^{\dagger} \hat{c}_{k} \hat{c}_{l} \hat{c}_{2}^{\dagger} \hat{c}_{1}^{\dagger} \hat{c}_{3} \hat{c}_{3}^{\dagger} \ldots \hat{c}_{N} \hat{c}_{N}^{\dagger} n_{i} n_{j} n_{k} n_{l} \\
& =\hat{c}_{1} \hat{c}_{i}^{\dagger} \hat{c}_{2} \hat{c}_{j}^{\dagger} \hat{c}_{k} \hat{c}_{2}^{\dagger} \hat{c}_{l} \hat{c}_{1}^{\dagger} n_{i} n_{j} n_{k} n_{l} \\
& +\hat{c}_{1} \hat{c}_{j}^{\dagger} \hat{c}_{2} \hat{c}_{i}^{\dagger} \hat{c}_{k} \hat{c}_{1}^{\dagger} \hat{c}_{l} \hat{c}_{2}^{\dagger} n_{i} n_{j} n_{k} n_{l} \\
& -\hat{c}_{1} \hat{c}_{i}^{\dagger} \hat{c}_{2} \hat{c}_{j}^{\dagger} \hat{c}_{k} \hat{c}_{1}^{\dagger} \hat{c}_{l} \hat{c}_{2}^{\dagger} n_{i} n_{j} n_{k} n_{l} \\
& -\hat{c}_{1} \hat{c}_{j}^{\dagger} \hat{c}_{2} \hat{c}_{i}^{\dagger} \hat{c}_{k} \hat{c}_{2}^{\dagger} \hat{c}_{l} \hat{c}_{1}^{\dagger} n_{i} n_{j} n_{k} n_{l} \\
& =\left(\delta_{i 1} \delta_{j 2} \delta_{k 2} \delta_{l 1}+\delta_{i 2} \delta_{j 1} \delta_{k 1} \delta_{l 2}-\delta_{i 1} \delta_{j 2} \delta_{k 1} \delta_{l 2}-\delta_{i 2} \delta_{j 1} \delta_{k 2} \delta_{l 1}\right) \\
& \times n_{i} n_{j} n_{k} n_{l} \\
& =\left(\delta_{i 1} \delta_{j 2} \delta_{i l} \delta_{j k}+\delta_{j 1} \delta_{i 2} \delta_{i l} \delta_{j k}-\delta_{i 1} \delta_{j 2} \delta_{i k} \delta_{j l}-\delta_{j 1} \delta_{i 2} \delta_{i k} \delta_{j l}\right) \\
& \times n_{i} n_{j} n_{k} n_{l} \\
& =\left(\delta_{i l} \delta_{j k}-\delta_{i k} \delta_{j l}\right)\left(\delta_{i 1} \delta_{j 2}+\delta_{j 1} \delta_{i 2}\right) n_{i} n_{j} n_{k} n_{l} \text {. }
\end{aligned}
$$

Because we can only have either $i=1, j=2$ or $i=2, j=1$, the result is

$$
\left\langle\Phi\left|\hat{c}_{i}^{\dagger} \hat{c}_{j}^{\dagger} \hat{c}_{k} \hat{c}_{l}\right| \Phi\right\rangle=\left(\delta_{i l} \delta_{j k}-\delta_{i k} \delta_{j l}\right) n_{i} n_{j}
$$

Next, we use the expansions

$$
\hat{c}_{a}^{\dagger}=\sum_{i} S_{i a} \hat{c}_{i}^{\dagger}
$$

and

$$
\hat{c}_{a}=\sum_{i} S_{i a}^{*} \hat{c}_{i}
$$

of the creation and annihilation operators, respectively, to transform the result into a different basis. The one-particle density matrix in the new basis can be expressed by the occupation numbers $n_{i}$ of the basis states for which the 
A.2 Expectation values of occupation number operators in the grand canonical ensemble for a non-interacting Hamiltonian

one-particle density matrix is diagonal,

$$
\begin{aligned}
\rho_{b a} & =\left\langle\Phi\left|\hat{c}_{a}^{\dagger} \hat{c}_{b}\right| \Phi\right\rangle \\
& =\sum_{i j} S_{i a} S_{j b}^{*}\left\langle\Phi\left|\hat{c}_{i}^{\dagger} \hat{c}_{j}\right| \Phi\right\rangle \\
& =\sum_{i j} S_{i a} S_{j b}^{*} n_{i} \delta_{i j} \\
& =\sum_{i} S_{i a} n_{i} S_{i b}^{*} .
\end{aligned}
$$

In the new basis, the expectation value of the Slater determinant becomes

$$
\begin{aligned}
\left\langle\Phi\left|\hat{c}_{a}^{\dagger} \hat{c}_{b}^{\dagger} \hat{c}_{c} \hat{c}_{d}\right| \Phi\right\rangle= & \sum_{i j k l} S_{i a} S_{j b} S_{k c}^{*} S_{l d}^{*}\left\langle\Phi\left|\hat{c}_{i}^{\dagger} \hat{c}_{j}^{\dagger} \hat{c}_{k} \hat{c}_{l}\right| \Phi\right\rangle \\
= & \sum_{i j k l} S_{i a} S_{j b} S_{k c}^{*} S_{l d}^{*}\left(\delta_{i l} \delta_{j k}-\delta_{i k} \delta_{j l}\right) n_{i} n_{j} \\
= & \sum_{i j} S_{i a} S_{j b} S_{j c}^{*} S_{i d}^{*} n_{i} n_{j}-\sum_{i j} S_{i a} S_{j b} S_{i c}^{*} S_{j d}^{*} n_{i} n_{j} \\
= & {\left[\sum_{i} S_{i a} n_{i} S_{i d}^{*}\right]\left[\sum_{j} S_{j b} n_{j} S_{j c}^{*}\right] } \\
& -\left[\sum_{i} S_{i a} n_{i} S_{i c}^{*}\right]\left[\sum_{j} S_{j b} n_{j} S_{j d}^{*}\right] \\
= & \rho_{d a} \rho_{c b}-\rho_{c a} \rho_{d b} .
\end{aligned}
$$

This completes the proof of eq. (A.4).

\section{A.2 | Expectation values of occupation number oper- ators in the grand canonical ensemble for a non- interacting Hamiltonian}

Consider a system of $N_{\text {orb }}$ fermionic orbitals $\left|\phi_{1}\right\rangle \ldots\left|\phi_{N_{\text {orb }}}\right\rangle$ with the noninteracting Hamiltonian

$$
\hat{H}_{0}=\sum_{k} \varepsilon_{k} \hat{a}_{k}^{\dagger} \hat{a}_{k}=\sum_{k} \varepsilon_{k} \hat{n}_{k}
$$

The operators $\hat{a}_{k}^{\dagger}$ and $\hat{a}_{k}$ create and annihilate a particle in a single-particle orbital $\left|\phi_{k}\right\rangle$, respectively. $\hat{n}_{k}=\hat{a}_{k}^{\dagger} \hat{a}_{k}$ is the occupation number operator for orbital $\left|\phi_{k}\right\rangle$. An eigenstate to the Hamiltonian (A.29) is a Slater determinant, which in occupation number representation can be written as $\left|n_{1}, \ldots, n_{N_{\text {orb }}}\right\rangle$. Each occupation number can be either 0 or 1 . Suppose the system can exchange energy and particles with a bath. In this case, we describe the system with a 
grand canonical ensemble. Our aim is to show

$$
\left\langle\hat{n}_{i} \hat{n}_{j}\right\rangle_{0}=\left\langle\hat{n}_{i}\right\rangle_{0}\left\langle\hat{n}_{j}\right\rangle_{0},
$$

where $\langle\cdot\rangle_{0}$ denotes the expectation value with respect to the grand canonical ensemble for a non-interacting Hamiltonian (A.29). Subsequently, this result is used in conjunction with the findings of the previous section to complete the proof for eq. (A.3).

A sum over all eigenstates of the Hamiltonian (A.29) can be expressed by a sum over all possible combinations of the occupation numbers of the individual one-particle orbitals. Hence, the grand canonical partition function can be written as

$$
\begin{aligned}
Z_{0} & =\operatorname{Tr}\left[e^{-\beta \sum_{k}\left(\varepsilon_{k}-\mu\right) \hat{n}_{k}}\right] \\
& =\sum_{n_{1} \in\{0,1\}} \cdots \sum_{n_{N_{\mathrm{orb}}} \in\{0,1\}} e^{-\beta \sum_{k}\left(\varepsilon_{k}-\mu\right) n_{k}} \\
& =\sum_{n_{1} \in\{0,1\}} \cdots \sum_{n_{\mathrm{orb}_{\mathrm{orb}} \in\{0,1\}}} \prod_{k} e^{-\beta\left(\varepsilon_{k}-\mu\right) n_{k}} \\
& =\prod_{k} \sum_{n_{k} \in\{0,1\}} e^{-\beta\left(\varepsilon_{k}-\mu\right) n_{k}} \\
& =\prod_{k}\left(1+e^{-\beta\left(\varepsilon_{k}-\mu\right) n_{k}}\right)
\end{aligned}
$$

Because each sum over an occupation number belongs to one factor of the product, the sum can be moved into the product.

In a similar fashion, we obtain the Fermi distribution function as a result for the expectation value of the occupation number operator for one of the single-particle orbitals:

$$
\begin{aligned}
\left\langle\hat{n}_{i}\right\rangle_{0} & =\sum_{n_{1} \in\{0,1\}} \cdots \sum_{n_{N_{\mathrm{orb}}} \in\{0,1\}}\left\langle n_{1}, \ldots, n_{N_{\mathrm{orb}}}\left|\frac{1}{Z_{0}} e^{-\beta \sum_{k}\left(\varepsilon_{k}-\mu\right) \hat{n}_{k}} \hat{n}_{i}\right| n_{1}, \ldots, n_{N_{\mathrm{orb}}}\right\rangle \\
& =\sum_{n_{1} \in\{0,1\}} \cdots \sum_{n_{N_{\mathrm{orb}}} \in\{0,1\}} \frac{1}{Z_{0}} e^{-\beta \sum_{k}\left(\varepsilon_{k}-\mu\right) n_{k}} n_{i} \\
& =\frac{1}{Z_{0}} \sum_{n_{1} \in\{0,1\}} \cdots \sum_{n_{N_{\mathrm{orb}}} \in\{0,1\}}\left[\prod_{k} e^{-\beta\left(\varepsilon_{k}-\mu\right) n_{k}}\right] n_{i} \\
& =\frac{1}{Z_{0}}\left[\prod_{k \neq i} \sum_{n_{k} \in\{0,1\}} e^{-\beta\left(\varepsilon_{k}-\mu\right) n_{k}}\right]\left[\sum_{n_{i} \in\{0,1\}} e^{-\beta\left(\varepsilon_{i}-\mu\right) n_{i}} n_{i}\right] \\
& =\frac{e^{-\beta\left(\varepsilon_{i}-\mu\right)}}{1+e^{-\beta\left(\varepsilon_{i}-\mu\right)}} \\
& =\frac{1}{1+e^{\beta\left(\varepsilon_{i}-\mu\right)}}
\end{aligned}
$$


A.2 Expectation values of occupation number operators in the grand canonical ensemble for a non-interacting Hamiltonian

Moreover, with the same procedure we find for the expectation value of a product of two occupation number operators:

$$
\begin{aligned}
\left\langle\hat{n}_{i} \hat{n}_{j}\right\rangle_{0} & =\sum_{n_{1} \in\{0,1\}} \ldots \sum_{n_{N_{\mathrm{orb}}} \in\{0,1\}}\left\langle n_{1}, \ldots, n_{N_{\mathrm{orb}}}\left|\frac{1}{Z_{0}} e^{-\beta \sum_{k}\left(\varepsilon_{k}-\mu\right) \hat{n}_{k}} \hat{n}_{i} \hat{n}_{j}\right| n_{1}, \ldots, n_{N_{\mathrm{orb}}}\right\rangle \\
& =\sum_{n_{1} \in\{0,1\}} \cdots \sum_{n_{N_{\mathrm{orb}}} \in\{0,1\}} \frac{1}{Z_{0}} e^{-\beta \sum_{k}\left(\varepsilon_{k}-\mu\right) n_{k}} n_{i} n_{j} \\
& =\frac{1}{Z_{0}} \sum_{n_{1} \in\{0,1\}} \cdots \sum_{n_{N_{\mathrm{orb}}} \in\{0,1\}}\left[\prod_{k} e^{-\beta\left(\varepsilon_{k}-\mu\right) n_{k}}\right] n_{i} n_{j} \\
& =\frac{1}{Z_{0}}\left[\prod_{k \neq i, j} \sum_{n_{k} \in\{0,1\}} e^{-\beta\left(\varepsilon_{k}-\mu\right) n_{k}}\right] \\
& =\frac{e^{-\beta\left(\varepsilon_{i}-\mu\right)}}{1+e^{-\beta\left(\varepsilon_{i}-\mu\right)}} \frac{e^{-\beta\left(\varepsilon_{j}-\mu\right)}}{1+e^{-\beta\left(\varepsilon_{j}-\mu\right)}} \\
& =\frac{1}{1+e_{n_{i} \in\{0,1\}} e^{-\beta\left(\varepsilon_{i}-\mu\right)} \frac{1}{1+e^{\beta\left(\varepsilon_{j}-\mu\right)}}} \\
& =\left\langle\hat{n}_{i}\right\rangle_{0}\left\langle\hat{n}_{j}\right\rangle_{0} \cdot
\end{aligned}
$$

We see that, in the case of a non-interacting Hamiltonian, the expectation value $\left\langle\hat{n}_{i} \hat{n}_{j}\right\rangle_{0}$ factorizes, which proves eq. (A.30).

The above result can be used to evaluate the expectation value $\left\langle\hat{c}_{a}^{\dagger} \hat{c}_{b}^{\dagger} \hat{c}_{c} \hat{c}_{d}\right\rangle_{\mathrm{HF}}$, which occurs in the derivation of the Hartree-Fock approximation. First, we use eq. (A.17) and eq. (A.48) to determine the expectation value $\left\langle\hat{c}_{i}^{\dagger} \hat{c}_{j}^{\dagger} \hat{c}_{k} \hat{c}_{l}\right\rangle_{\mathrm{HF}}$ in the single-particle eigenbasis $\{|\phi\rangle\}$ of the matrix $h_{a b}$ :

$$
\begin{aligned}
\left\langle\hat{c}_{i}^{\dagger} \hat{c}_{j}^{\dagger} \hat{c}_{k} \hat{c}_{l}\right\rangle_{\mathrm{HF}} & =\left(\delta_{i l} \delta_{j k}-\delta_{i k} \delta_{j l}\right)\left\langle\hat{n}_{i} \hat{n}_{j}\right\rangle_{\mathrm{HF}} \\
& =\left(\delta_{i l} \delta_{j k}-\delta_{i k} \delta_{j l}\right)\left\langle\hat{n}_{i}\right\rangle_{\mathrm{HF}}\left\langle\hat{n}_{j}\right\rangle_{\mathrm{HF}} .
\end{aligned}
$$

We then transform the result into the basis $\{|\varphi\rangle\}$ :

$$
\begin{aligned}
\left\langle\hat{c}_{a}^{\dagger} \hat{c}_{b}^{\dagger} \hat{c}_{c} \hat{c}_{d}\right\rangle_{\mathrm{HF}}= & \sum_{i j k l} S_{i a} S_{j b} S_{k c}^{*} S_{l d}^{*}\left\langle\hat{c}_{i}^{\dagger} \hat{c}_{j}^{\dagger} \hat{c}_{k} \hat{c}_{l}\right\rangle_{\mathrm{HF}} \\
= & \sum_{i j k l} S_{i a} S_{j b} S_{k c}^{*} S_{l d}^{*}\left(\delta_{i l} \delta_{j k}-\delta_{i k} \delta_{j l}\right)\left\langle\hat{n}_{i}\right\rangle_{\mathrm{HF}}\left\langle\hat{n}_{j}\right\rangle_{\mathrm{HF}} \\
= & \sum_{i j} S_{i a} S_{j b} S_{j c}^{*} S_{i d}^{*}\left\langle\hat{n}_{i}\right\rangle_{\mathrm{HF}}\left\langle\hat{n}_{j}\right\rangle_{\mathrm{HF}} \\
& \quad-\sum_{i j} S_{i a} S_{j b} S_{i c}^{*} S_{j d}^{*}\left\langle\hat{n}_{i}\right\rangle_{\mathrm{HF}}\left\langle\hat{n}_{j}\right\rangle_{\mathrm{HF}}
\end{aligned}
$$


A Supplemental material on the derivation of the Hartree-Fock approximation

$$
\begin{aligned}
= & {\left[\sum_{i} S_{i a}\left\langle\hat{n}_{i}\right\rangle_{\mathrm{HF}} S_{i d}^{*}\right]\left[\sum_{j} S_{j b}\left\langle\hat{n}_{j}\right\rangle_{\mathrm{HF}} S_{j c}^{*}\right] } \\
& -\left[\sum_{i} S_{i a}\left\langle\hat{n}_{i}\right\rangle_{\mathrm{HF}} S_{i c}^{*}\right]\left[\sum_{j} S_{j b}\left\langle\hat{n}_{j}\right\rangle_{\mathrm{HF}} S_{j d}^{*}\right] \\
= & \left\langle\hat{c}_{a}^{\dagger} \hat{c}_{d}\right\rangle_{\mathrm{HF}}\left\langle\hat{c}_{b}^{\dagger} \hat{c}_{c}\right\rangle_{\mathrm{HF}}-\left\langle\hat{c}_{a}^{\dagger} \hat{c}_{c}\right\rangle_{\mathrm{HF}}\left\langle\hat{c}_{b}^{\dagger} \hat{c}_{d}\right\rangle_{\mathrm{HF}} .
\end{aligned}
$$

We insert this result into the expectation value for the interaction operator $\langle\hat{W}\rangle_{\mathrm{HF}}$ and, after relabeling the indices, we arrive at eq. (A.3). 


\section{B Atomic species setups for the CP-PAW simulation code}

In this section we list the setup parameters of the atomic species $\mathrm{B}, \mathrm{La}, \mathrm{Ce}, \mathrm{Pr}$, and $\mathrm{Nd}$ within the . strc input file for the CP-PAW code, that have been used in our DFT simulations. Values of NPRO for the set of projector functions and LHFWEIGHT for the species-dependent exchange mixing in the PBE0r hybrid functional are example values.

Setup parameters for boron:

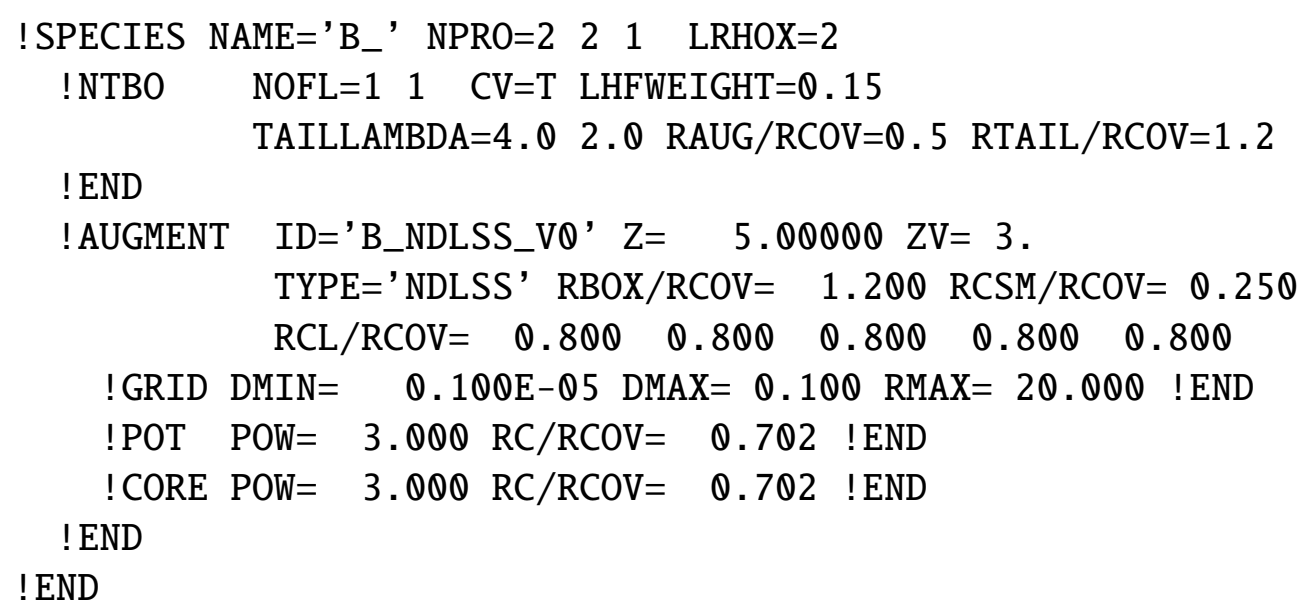

Setup parameters for lanthanum:

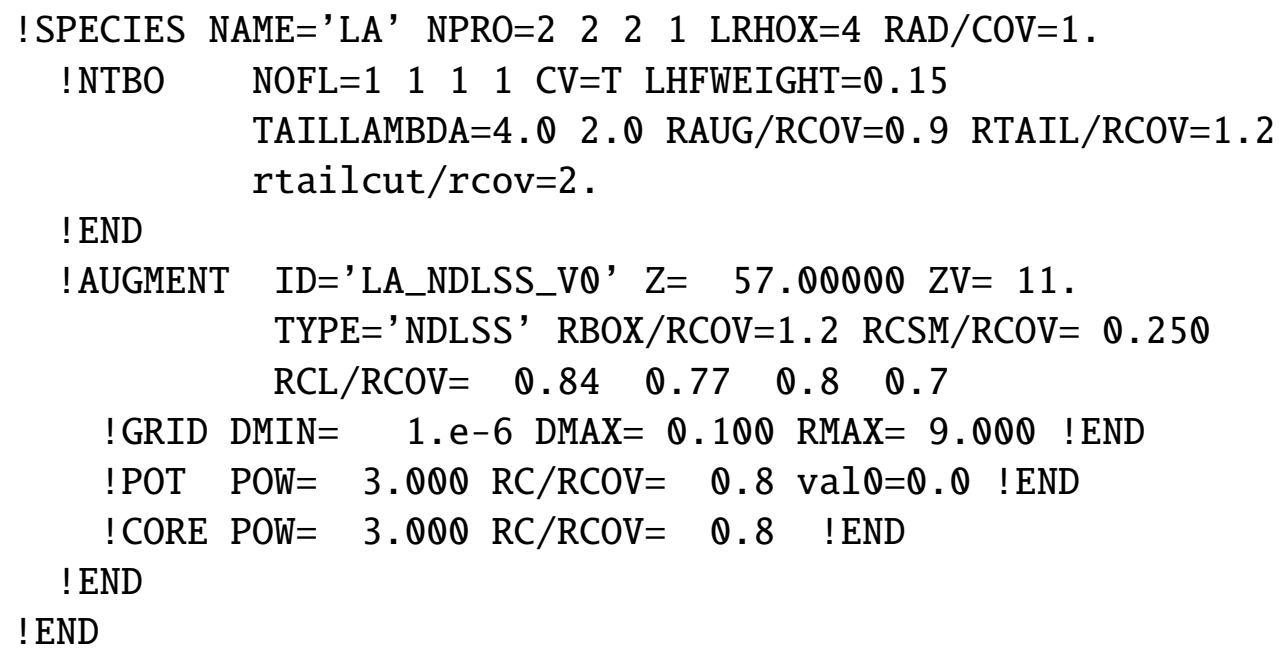


Setup parameters for cerium:

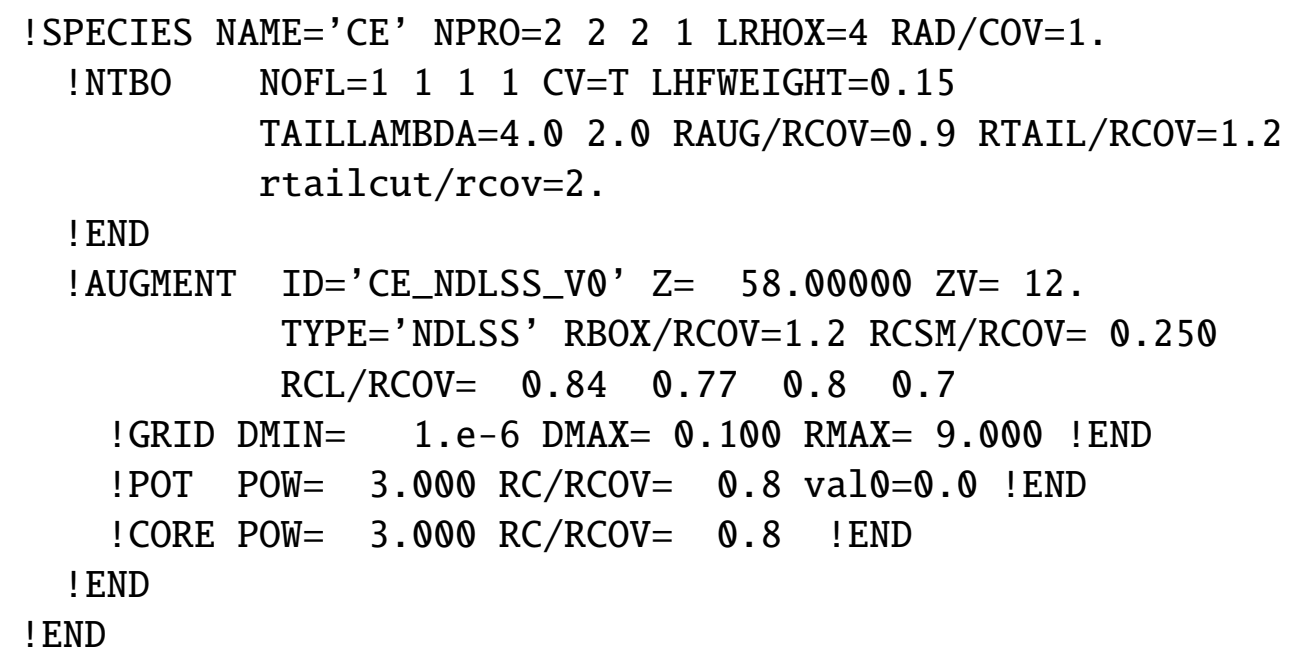

Setup parameters for praseodymium:

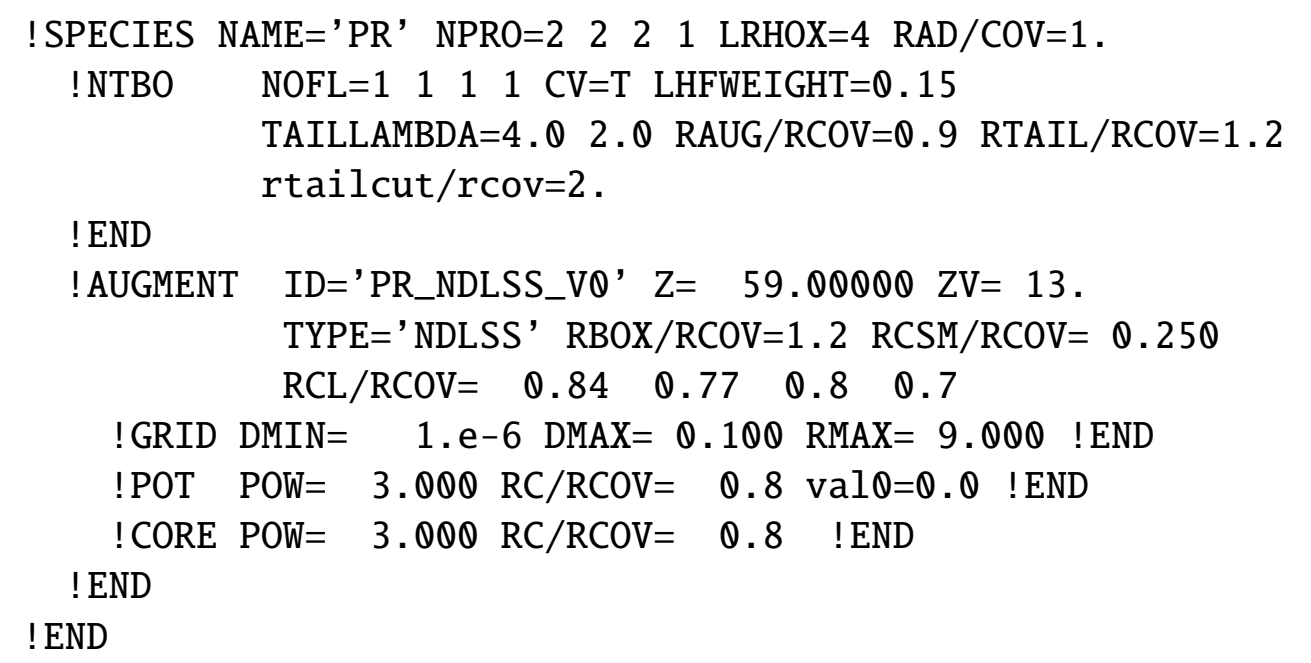

Setup parameters for neodymium:

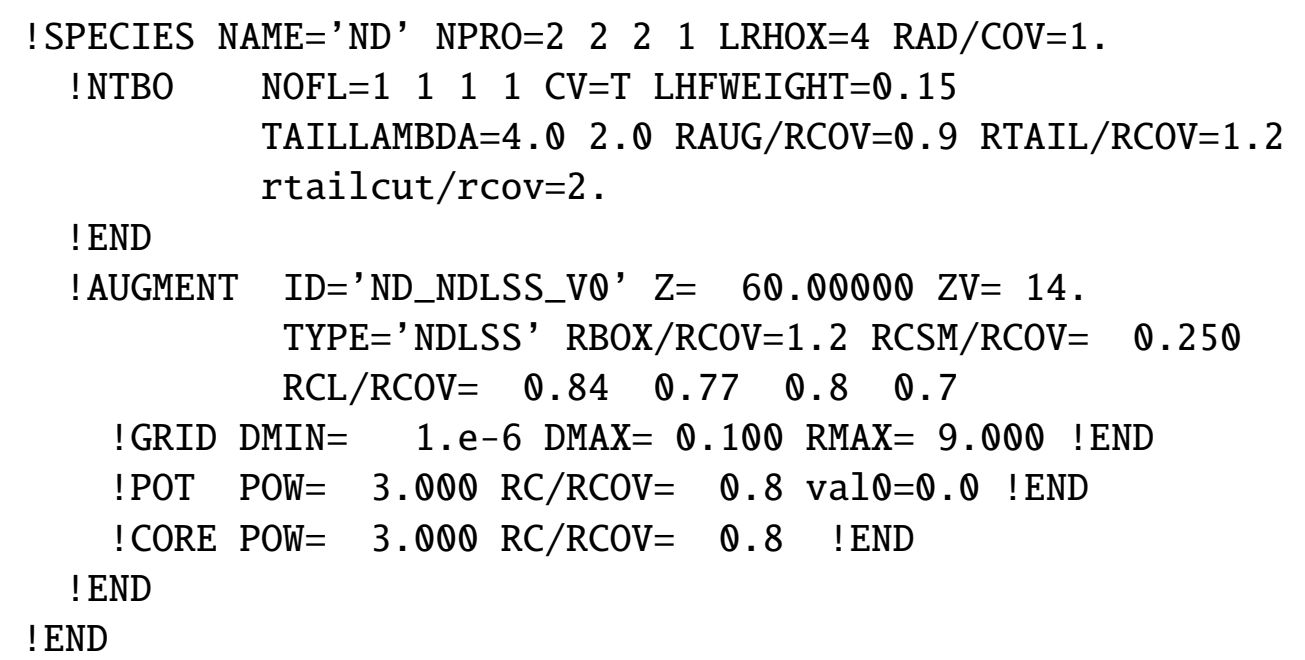


Supplemental information on the simulations of $\mathrm{PrB}_{6}$

\begin{tabular}{|c|c|c|c|}
\hline \multicolumn{4}{|c|}{$\vec{k}$-points: $(7 \times 7 \times 7)$} \\
\hline & TYPE I & Type II & TYPE III \\
\hline$(1,1,1)(2,1,1,1)$ & -65.16441 & -65.18034 & 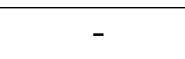 \\
\hline$(1,1,1)(2,2,1,1)$ & -65.15523 & -65.17239 & -65.17227 \\
\hline$(1,1,1)(2,2,2,1)$ & -65.16074 & -65.17803 & -65.17803 \\
\hline$(2,2,1)(2,2,2,1)$ & -65.23710 & - & -65.25484 \\
\hline \multicolumn{4}{|c|}{$\vec{k}$-points: $(9 \times 9 \times 9)$} \\
\hline & TyPe I & TyPE II & Tyре III \\
\hline$(1,1,1)(2,1,1,1)$ & -65.16438 & -65.18043 & 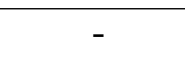 \\
\hline$(1,1,1)(2,2,1,1)$ & -65.15519 & -65.17242 & -65.17231 \\
\hline$(1,1,1)(2,2,2,1)$ & -65.16069 & -65.17804 & -65.17799 \\
\hline$(2,2,1)(2,2,2,1)$ & -65.23704 & - & -65.25479 \\
\hline \multicolumn{4}{|c|}{$\vec{k}$-points: $(11 \times 11 \times 11)$} \\
\hline & TyPe I & Type II & Type III \\
\hline$(1,1,1)(2,1,1,1)$ & -65.16440 & -65.18043 & - \\
\hline$(1,1,1)(2,2,1,1)$ & -65.15512 & -65.17234 & - \\
\hline$(1,1,1)(2,2,2,1)$ & -65.16062 & -65.17797 & -65.17797 \\
\hline$(2,2,1)(2,2,2,1)$ & -65.23697 & - & -65.25473 \\
\hline
\end{tabular}

\begin{tabular}{c|c|c|c}
$\vec{k}$-points: $(13 \times 13 \times 13)$ \\
& TYPE I & TYPE II & TYPE III \\
\hline$(1,1,1)(2,1,1,1)$ & -65.16438 & -65.18039 & - \\
$(1,1,1)(2,2,1,1)$ & -65.15509 & -65.17233 & - \\
$(1,1,1)(2,2,2,1)$ & -65.16057 & -65.17796 & -65.17795 \\
$(2,2,1)(2,2,2,1)$ & -65.23692 & - & -65.25472
\end{tabular}

Table C.1: Compilation of total energies from simulations of $\operatorname{PrB}_{6}$ with the PBE0r hybrid functional at an exchange mixing factor of 0.15 . TYPE I-III label the converged final states according to fig. 4.11. The rows are labeled with the sets of projector function for the boron $(s, p, d)$ and praseodymium $(s, p, d, f)$ states. Energies in Hartree. 



\section{Study of the radial extent of the $5 s, 5 p$, and $4 f$ orbitals of the $\mathrm{La}^{3+}$ ion}

The results of our DFT bulk simulations of the rare-earth hexaborides, which are presented in chapter 4 , show downward shifts of the rare earth $5 s$ and $5 p$ energy levels when switching from the PBE functional to the PBE0r hybrid functional. The shift width increases with larger values of the exchange mixing factor in the PBE0r hybrid functional. As discussed in chapter 4 and confirmed in the model analysis of chapter 5 this downward shift in energy is due to the correction of the self-interaction error in the PBE0r hybrid functional.

Moreover, we also observe that the unoccupied rare earth $4 f$ levels are shifted to higher energies. Again, the shift width becomes wider the larger we set the exchange mixing factor in the PBE0r hybrid functional. The compensation of a self-interaction error in the $4 f$ shell through the exact exchange term does not appear to be a plausible rationale for the upward shift in energy from a simple mean-field perspective, since a self-interaction only occurs in the case of occupied orbitals. However, this does not rule out that the exact exchange could affect the $4 f$ states indirectly. For instance, the subtraction of the selfinteraction can affect the spatial extension of the rare earth $5 s$ and $5 p$ states. In turn, the $4 f$ states could contract in response to the new environment. This would increase the interaction strength between the $4 f$ states and could result in the observed upward shift.

Following the reasoning of the preceding paragraph, it is important to check what effect the admixture of exact exchange in the PBE0r hybrid functional has on the size of the localized orbitals of the rare-earth ion in comparison to the results with the PBE functional. This is investigated in the following. At this occasion we like to mention that the hypothesis of spatially contracting $4 f$ states will be falsified, as no change in the spatial extension of the $4 f$ states is observed when switching from the PBE functional to the PBE0r hybrid functional. The most plausible origin of the increase in the $4 f$ energies appears to be the the double counting term of the PBE0r hybrid functional as discussed in section 5.9.2.

In this appendix we focus on lanthanum as rare-earth constituent. Since the $\mathrm{LaB}_{6}$ crystal is a rather complicated system, we instead study the simpler system of a single $\mathrm{La}^{3+}$ ion in a homogeneous negative charge background. In the $\mathrm{La}^{3+}$ ion the occupied valence orbitals are the fully occupied $5 s$ and $5 p$ orbitals. The system is spin-degenerate and also the individual orbitals within the $5 s$, the $5 p$, the $4 f$, and the $5 d$ shells are degenerate. We simulate the system with the PBE functional and with the PBE0r functional at an exchange mixing factor of 0.2. Our unit cell repeats along the face-centered cubic lattice vectors $(0, a, a),(a, 0, a),(a, a, 0)$, where $a=9 \AA$. For the La constituent we take the 
atomic setup given in appendix B. The plane-wave cutoff is set to 50 Ry for the wave functions and to 200 Ry for the density.

The Kohn-Sham wave functions have the shapes of atomic wave functions and their energy levels show the expected degeneracies. For the $5 s, 5 p$, and $4 f$ states, which are the most localized valence states, we find the energies listed in table D.1. Concordant with the simulations of $\mathrm{LaB}_{6}$ we find downward shifts of the $5 s$ and $5 p$ levels and an upward shift of the $4 f$ levels when switching from the PBE functional to the PBE0r hybrid functional. The $5 s$ orbitals have the same shift width in the $\mathrm{LaB}_{6}$ crystal and the $\mathrm{La}^{3+}$ ion. This can be rationalized by the fact that these states are far below other energy levels and a hybridization with other orbitals of the $\mathrm{LaB}_{6}$ crystal does not occur. As for the $5 p$ states, the smaller energetic difference in the $\mathrm{LaB}_{6}$ system is likely due to a small hybridization with boron $s$ states, see fig. 4.7. For the $4 f$ orbitals the shift width differs by a factor of 2 , i. e. $0.7 \mathrm{eV}$, between the $\mathrm{LaB}_{6}$ crystal and the $\mathrm{La}^{3+}$ ion. Hence, the impact of the La ion's surroundings in the $\mathrm{LaB}_{6}$ crystal onto the $4 f$ levels appears to be significant.

\begin{tabular}{l|c|c|c} 
& $5 s$ & $5 p$ & $4 f$ \\
\hline PBE functional & $-50.65 \mathrm{eV}$ & $-33.71 \mathrm{eV}$ & $-16.43 \mathrm{eV}$ \\
PBE0r functional & $-54.52 \mathrm{eV}$ & $-36.51 \mathrm{eV}$ & $-15.76 \mathrm{eV}$ \\
\hline difference & $-3.87 \mathrm{eV}$ & $-2.80 \mathrm{eV}$ & $+0.67 \mathrm{eV}$ \\
\hline difference in $\mathrm{LaB}_{6}$ & $-3.9 \mathrm{eV}$ & $-2.5 \mathrm{eV}$ & $+1.4 \mathrm{eV}$
\end{tabular}

Table D.1: Energy levels of the $5 s, 5 p$, and $4 f$ orbitals from our simulations of the $\mathrm{La}^{3+}$ in a homogeneous negative charge background. First row: simulation with the PBE functional. Second row: simulation with the PBE0r hybrid functional at an exchange mixing factor of 0.2. Third row: difference PBE-PBE0r data. Forth row: same difference for the features in the projected DOS from simulations of $\mathrm{LaB}_{6}$ with read-off errors of $0.1 \mathrm{eV}, 0.2 \mathrm{eV}$, and $0.2 \mathrm{eV}$ for the $5 s$, $5 p$, and $4 f$ features, respectively.

Because the Kohn-Sham wave functions have the shape of atomic wave functions, we can compare the radial extent of the most localized $5 s, 5 p$, and $4 f$ orbitals by taking the following approach. We write $n_{l m}(\vec{r})$ for the density that comes from an atomic wave function $\psi_{l m}(\vec{r})$ with quantum number of angular momentum $l$ and magnetic quantum number $m$. The density $n_{l m}(\vec{r})$ can be split into a radial contribution and an angular contribution,

$$
n_{l m}(\vec{r})=\left|\psi_{l m}(\vec{r})\right|^{2}=\left|R_{l}(r)\right|^{2}\left|Y_{l m}(\vartheta, \varphi)\right|^{2} .
$$

$R_{l}(r)$ is the radial function, and $Y_{l m}(\vartheta, \varphi)$ denotes a spherical harmonic. The spherical harmonics have the property (see e.g. [117])

$$
\sum_{m=-l}^{l}\left|Y_{l m}(\vartheta, \varphi)\right|^{2}=\frac{2 l+1}{4 \pi}
$$

which can be used to find the following relation between the density $n_{l m}(\vec{r})$ and 
the radial function $R_{l}(r)$ :

$$
\frac{4 \pi}{2 l+1} \sum_{m=-l}^{l} n_{l m}(\vec{r})=\left|R_{l}(r)\right|^{2} .
$$

We obtain the squares of the radial functions for the $5 s, 5 p$, and $4 f$ orbitals by computing the densities coming from each of the relevant Kohn-Sham wave functions and summing over the magnetic quantum number. This gives the left-hand side of eq. (D.3). Differences in the radial extents of the respective Kohn-Sham wave functions from the simulation with the PBE functional and from the simulations with the PBE0r hybrid functional at an exchange mixing factor of 0.2 are revealed in a comparison of the radial function data. Such comparisons are shown in fig. D. 1 for the $5 s, 5 p$, and $4 f$ orbitals respectively. Furthermore, the associated radial probability distributions are depicted in fig. D.2. Note that we first compute the density data on a three-dimensional cubic grid in real space with a grid spacing of 0.025 Bohr radii via a Fourier interpolation of the DFT Kohn-Sham data. Subsequently, we set up a radial grid of $50 \times 50$ angular coordinates $(\vartheta, \varphi)$ with equally spaced angles $\vartheta$ and $\varphi$. Further, we use a set of radial coordinates $r$ with a spacing of $0.01 \mathrm{Bohr}$ radii. We then employ a trilinear interpolation to obtain values for the density at the coordinates of the radial grid. For each radial coordinate we compute the mean value over the set of angular coordinates. In each plot we also show the square of the all-electron partial wave of the PAW method that corresponds to the respective radial function.

In fig. D. 1 we see that the squares of the radial functions as calculated from the Kohn-Sham densities follow the graphs of the all-electron partial wave functions. This means that the Kohn-Sham wave functions are close to the atomic wave functions of the respective orbitals. Close to the origin, the data of $\left|R_{l}(r)\right|^{2}$, with $l=s, p$, from the Kohn-Sham densities deviates from that of the all-electron partial wave function because the strong oscillations of the atomic wave functions are not reproduced in detail by the Fourier interpolation. However, the curves are in good agreement beyond $r=0.3$ Bohr radii. In comparison to the data from the simulation with the PBE functional, the data from the simulation with the PBE0r hybrid functional is smaller at small $r$ and slightly larger at large $r$. This indicates a slightly larger spatial extent of the $5 s$-like and $5 p$-like Kohn-Sham states when using the PBE0r functional, see also fig. D.2. The curves for the $4 f$ orbitals are virtually identical, exhibiting no change in the spatial extent of the $4 f$ states when switching on a quite large exchange mixing with a mixing factor of 0.2 .

The data in figs. D. 1 and D. 2 show that switching from the PBE functional to the PBE0r hybrid functional does not lead to a contraction of the $4 f$ states. Hence, based on our findings, we can exclude that an increase of the interaction in the $4 f$ shell due to a contraction of the $4 f$ states leads to the upward shift of the $4 f$ levels. The most plausible rationale for the upward shift of the unoccupied $4 f$ levels appears to be the subtraction of the double counting term in the PBE0r hybrid functional as detailed in section 5.9.2. 

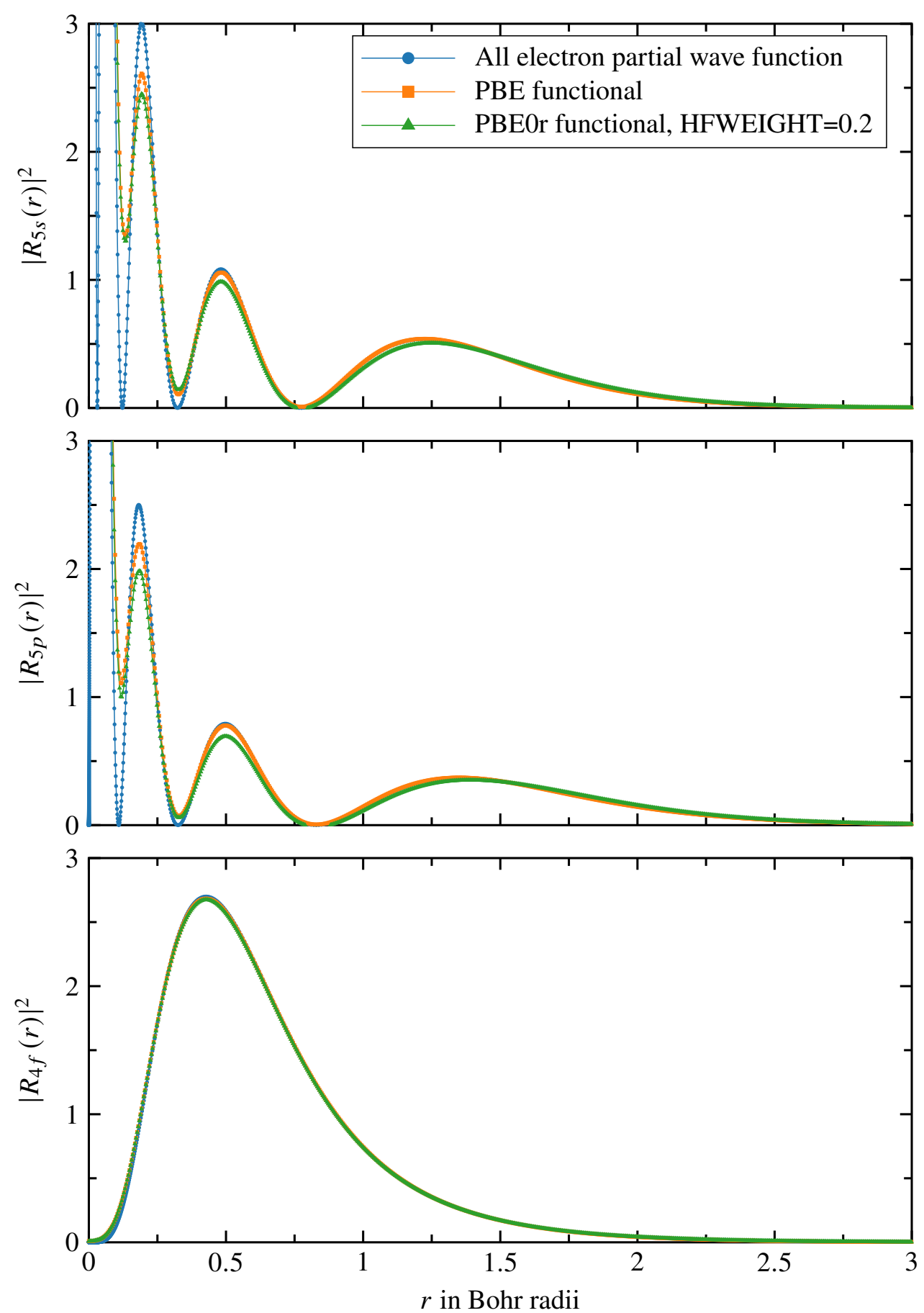

Figure D.1: Squares of the radial functions of the $5 s$ (top), $5 p$ (center), and $4 f$ (bottom) orbitals of a $\mathrm{La}^{3+}$ ion, obtained with the PAW method and DFT. Details are given in the text. For each orbital shell we show the square of the all electron partial wave function from the PAW method, the square of the radial function obtained with the PBE functional, and the square of the radial function obtained with the PBE0r hybrid functional at an exchange mixing factor HFWEIGHT $=0.2$. 

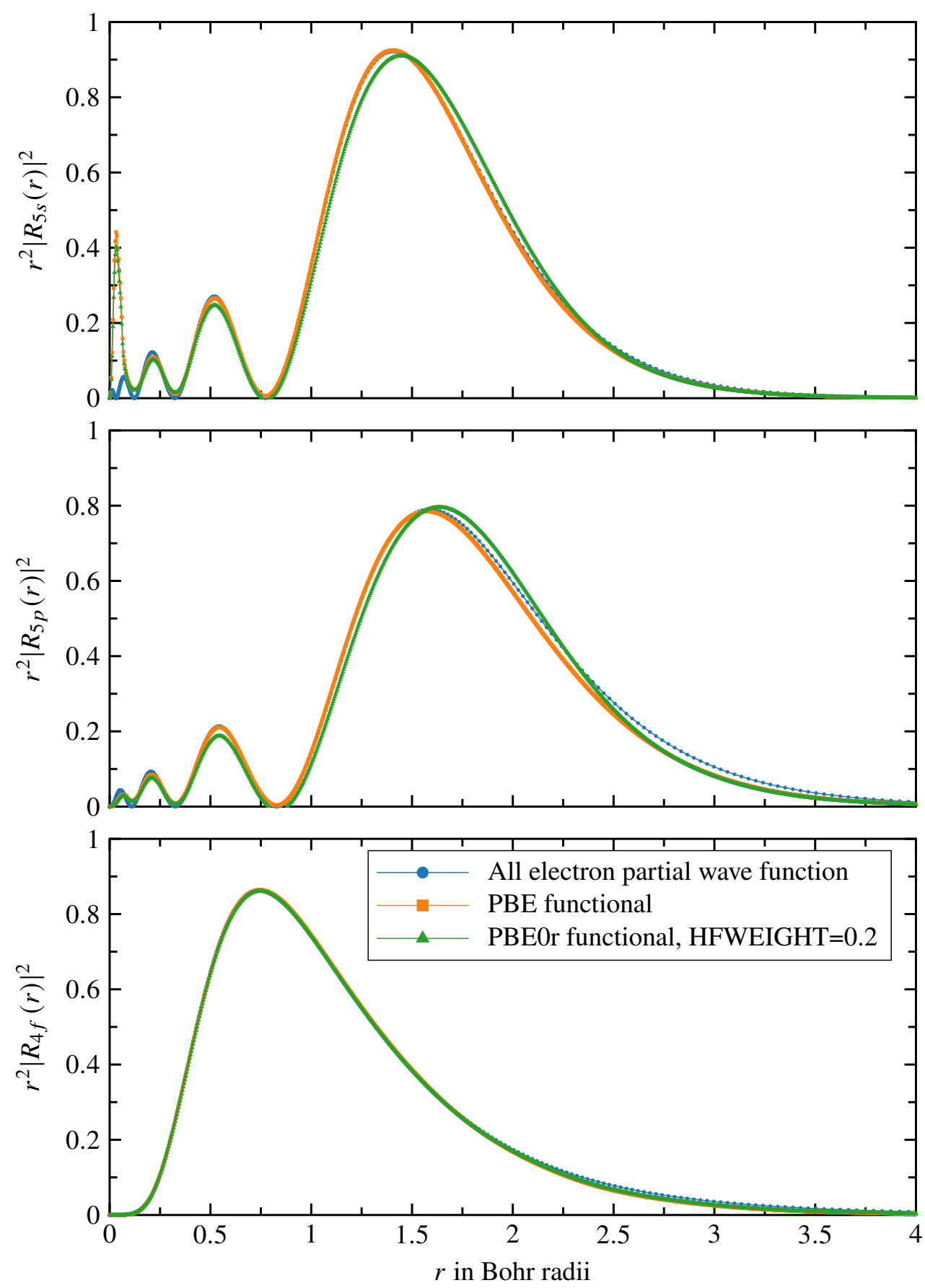

Figure D.2: Radial probability distribution functions from the radial data in fig. D.1. 



\section{Additional material on the scanning tunneling spectra}

In scanning tunneling microscopy (STM) and spectroscopy, a fundamental quantity is the tunneling barrier between the STM tip and the sample. It depends on the atomic and electronic structures of both, the tip and the sample. In appendix E.1, we briefly present the definition of the so-called apparent barrier height, which is a measure for the tunneling barrier between sample and tip, derived from a simple one-dimensional model.

For our study of the $\mathrm{LaB}_{6}$ (001) cleavage plane, spatially resolved scanning tunneling spectra have been recorded quasi-simultaneously to the constant current topography by our experimental collaborators. In the measurement procedure, the STM tip is generally not at the same height for consecutive measurements of the scanning tunneling spectra because it follows the contour of the constant current topography. A topography normalization is applied to the scanning tunneling spectra by our experimental collaborators to approximately project the spectra onto a plane of constant height. For this, the apparent barrier height is employed. The topography normalization is explained in appendix E.2.

We also show the constant current topography, the apparent barrier height, a spatially resolved scanning tunneling spectrum, and the area-averaged spectrum for a second data set, that was recorded to resolve the energy range close to the Fermi energy.

\section{E.1 The apparent barrier height}

The apparent barrier height is usually defined with the help of the simple one-dimensional model of section 3.13.1, which approximately captures the tunneling process. Within the model, the sample surface is located at position $z_{0}$ and the tip apex at position $z_{1}$. The system is governed by the potential (3.205), which represents a constant tunneling barrier between the tip and the sample and is zero otherwise. If the tip states are neglected, the tunneling current between the sample and the tip is proportional to the sum of the tunneling probabilities eq. (3.210) of the states at the sample surface close to the Fermi level $E_{\mathrm{F}}[124]$,

$$
I \propto \sum_{\substack{\mu \\ E_{\mu} \in\left[E_{\mathrm{F}}-e V, E_{\mathrm{F}}\right]}} P_{\mu}\left(z_{1}\right)=\sum_{\substack{\mu \\ E_{\mu} \in\left[E_{\mathrm{F}}-e V, E_{\mathrm{F}}\right]}}\left|\psi_{\mu}\left(z_{0}\right)\right|^{2} e^{-\sqrt{8 m} \sqrt{\phi} \Delta z / \hbar} .
$$


Here, $\mu$ labels the sample states, $V$ is the bias voltage, $m$ is the electron mass, $\phi$ is the sample work function, $\Delta z=z_{1}-z_{0}$ is the tip-sample distance, and $E_{\mu}$ denotes the energy eigenvalue of the wave function $\psi_{\mu}$. After insertion of a $\delta$-function eq. (E.1) becomes

$$
I \propto \int \mathrm{d} \varepsilon e^{-\sqrt{8 m} \sqrt{\phi} \Delta z / \hbar} \sum_{E_{\mu} \in\left[E_{\mathrm{F}}-e V, E_{\mathrm{F}}\right]}\left|\psi_{\mu}\left(z_{0}\right)\right|^{2} \delta\left(\varepsilon-E_{\mu}\right) .
$$

With the definition (3.204) of the local density of states (LDOS) of the sample $D_{\text {loc }}^{\mathrm{S}}\left(z_{0}, \varepsilon\right)$, we find

$$
I \propto \int_{E_{\mathrm{F}}-e V}^{E_{\mathrm{F}}} \mathrm{d} \varepsilon e^{-\sqrt{8 m} \sqrt{\phi} \Delta z / \hbar} D_{\mathrm{loc}}^{\mathrm{S}}\left(z_{0}, \varepsilon\right) .
$$

For an LDOS at the sample surface, that is approximately constant for typical values of the bias voltage $V$, i.e. $D_{\mathrm{loc}}^{\mathrm{S}}\left(z_{0}, \varepsilon\right) \approx D_{\mathrm{loc}}^{\mathrm{S}}\left(z_{0}, E_{\mathrm{F}}\right)$ for $\varepsilon \in\left[E_{\mathrm{F}}-\right.$ $\left.e V, E_{\mathrm{F}}\right]$, we can execute the integration to find

$$
I \propto e^{-\sqrt{8 m} \sqrt{\phi} \Delta z / \hbar} D_{\mathrm{loc}}^{\mathrm{S}}\left(z_{0}, E_{\mathrm{F}}\right) V .
$$

Within simple model, $\phi$ denotes the sample work function, which at the same time is a measure for the tunneling barrier. In real STM measurements the potential is more complicated, than in the simple model and both the tip and the sample possess individual work functions. If we assume that the tunneling current of the real system is still of the form (E.4), we have to replace the sample work function $\phi$ by the apparent barrier height $\phi_{\text {app }}$, which represents an effective tunneling barrier of the real system. The apparent barrier height is defined as

$$
\phi_{\text {app }}=\frac{\hbar^{2}}{8 m}\left[\frac{\mathrm{d} \ln I}{\mathrm{~d}(\Delta z)}\right]^{2} .
$$

The apparent barrier height can be measured in the following way. From a position above the surface, the tip is moved vertically towards the surface and simultaneously the tunneling current is recorded. The apparent barrier height is then computed via eq. (E.5) from the tip movement and the tunneling current data. In practice the measurement of the apparent barrier height can be combined with the quasi-simultaneous measurements of the constant current topography and the spatially resolved $I(V)$ data, that is described in section 3.13.6. At each measurement location, while the feedback loop for the tip height adjustment is deactivated, i. e. before or after the measurement of the $I(V)$ curve, the apparent barrier height can be measured as described above. 


\section{E.2 The topography normalization for spatially re- solved scanning tunneling spectra}

The scanning tunneling spectroscopy data presented in fig. 6.6 was recorded quasi-simultaneously to the constant current topography as described in section 3.13.6. Because the tip follows the constant current topography contour, individual measurements of the tunneling spectra are not performed at the same tip height. If tunneling spectra at the same tip height are desired, e.g. for a comparison to theoretical data, the spatially resolved tunneling spectra can be projected onto a two-dimensional plane of constant height via a topography normalization.
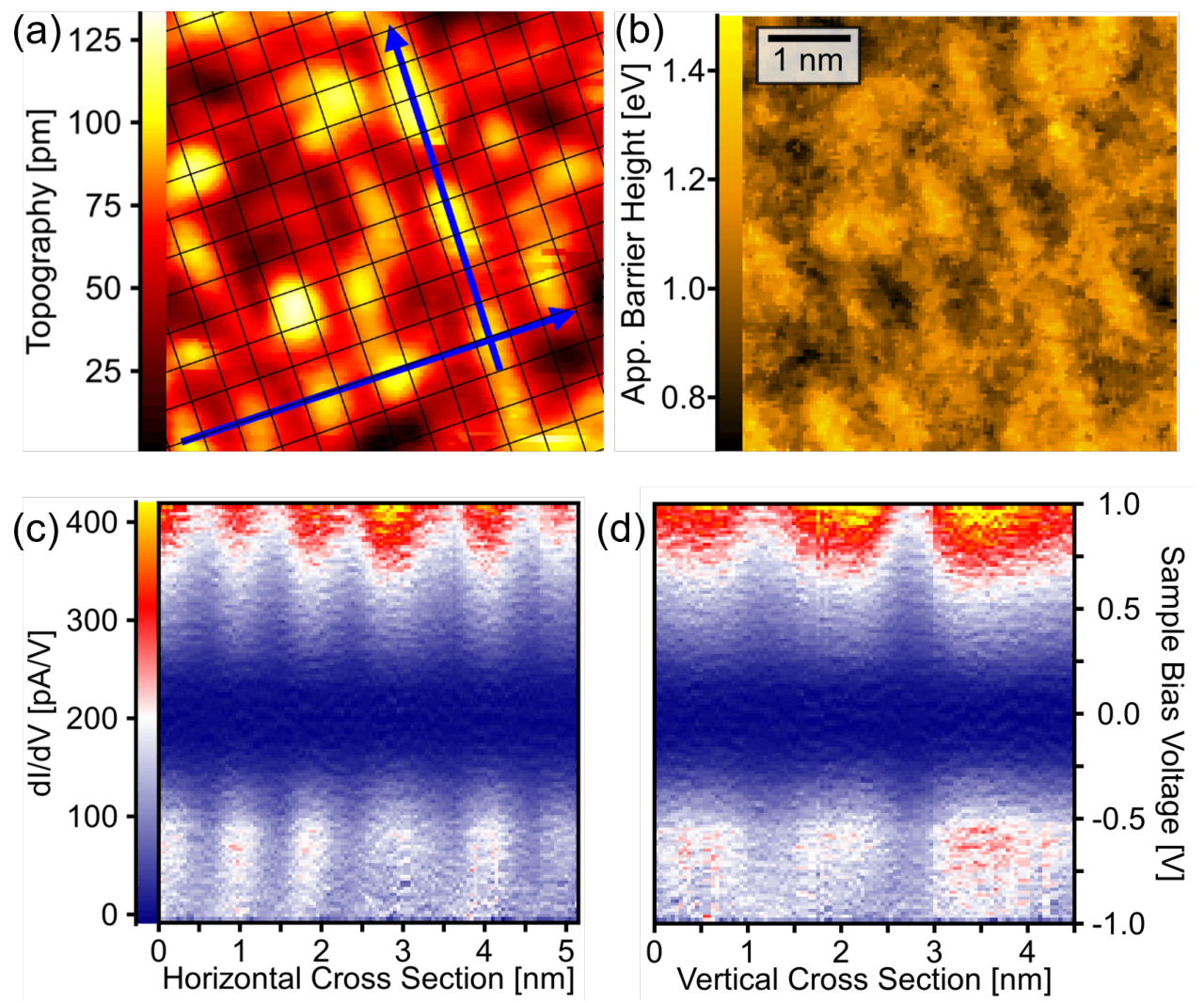

Figure E.1: (a) Same CCT as in fig. 6.6(a). An additional grid with a spacing of one lattice constant in both directions is added to visualize the lattice periodicity. Spatial dependences of the ST spectra along the sideward and upward pointing arrows are depicted in (c) and (d), respectively. (b) Spatially resolved apparent barrier height for the region in (a). Figure originally published in [157]. Reprinted with permission from the American Physical Society. (C) (2019) American Physical Society.

For the spatially resolved scanning tunneling spectra, a topography normalization can be performed by multiplying the measured tunneling current with the exponential $\exp \left[+\sqrt{8 m \phi_{\text {app,avg }}} \zeta(x, y) / \hbar\right](\mathrm{cf}$. [165]), where $\zeta(x, y)$ 
is the topography and $\phi_{\text {app,avg }}$ is the map average of the spatially resolved apparent barrier height. In this way, within the approximation of the simple one-dimensional tunneling theory of appendix E.1, the topography normalization approximately cancels the exponential dependence of the tunneling current on a displacement of the tip from a constant reference height. Effectively, the scanning tunneling spectroscopy data is approximately projected onto a plane of constant height. Such a topography normalization was applied to the data presented in fig. 6.6 and in the remainder of this appendix.

In fig. E.1(a) the constant current topography of fig. 6.6(a) is shown again, together with the spatially resolved apparent barrier height of the same surface area in fig. E.1(b). A topography normalization was performed and two spatially resolved tunneling spectra were obtained along the arrows indicated in fig. E.1(a). The resulting spectra are depicted in fig. E.1(c) and (d). It is clear that the largest values of the $\mathrm{d} I / \mathrm{d} V$ data for both, the negative as well as the positive bias voltage ranges, are found at the positions of the protrusions in the constant current topography. Importantly, increased $\mathrm{d} I / \mathrm{d} V$ values are found at the protrusions at approximately $-0.6 \mathrm{eV}$ sample bias voltage.

Scanning tunneling spectroscopy data of a second data set is shown in fig. E.2. Figure E.2(a) shows a constant current topography of a surface area with a chainlike $(2 \times 1)$ surface reconstruction. The apparent barrier height of this surface section is depicted in fig. E.2(b). Figure E.2(c) shows the spatially resolved tunneling spectrum along the arrow in fig. E.2(a) that is obtained after a topography normalization. Increased values of the $\mathrm{d} I / \mathrm{d} V$ data are found at the position of the protruding chains. The average tunneling spectrum of the surface area in fig. E.2(a) is plotted in fig. E.2(d). This plot is identical to the plot in fig. 6.6(d). It shows a $\mathrm{d} I / \mathrm{d} V$ curve, that is non-zero over the entire sample bias voltage range. Between $0.0 \mathrm{~V}$ and $0.1 \mathrm{~V}$, a feature is indicated by the raised shape of the curve. 
(a)

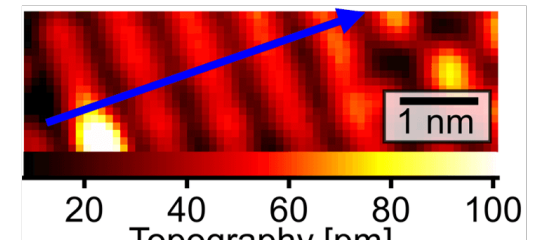

(c)
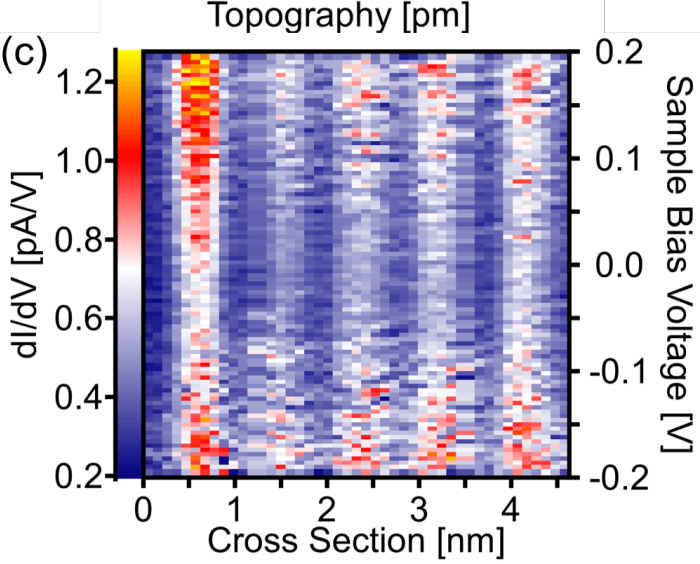

(b)

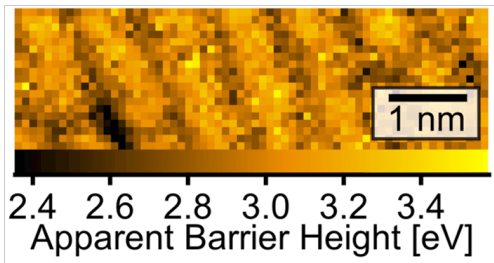

(d)

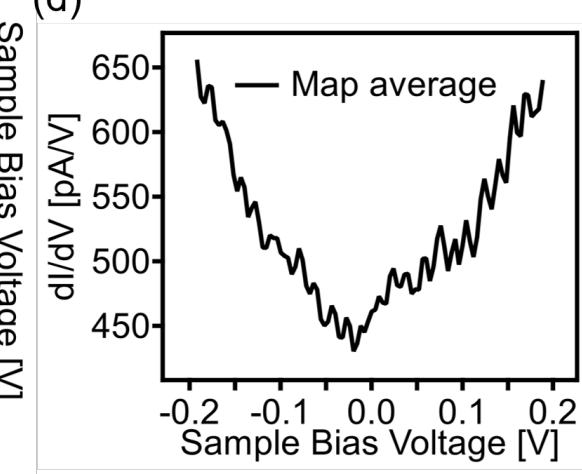

Figure E.2: (a) CCT of a surface area, that was selected for STS measurements. bias voltage: $0.2 \mathrm{~V}$, tunneling current: $0.1 \mathrm{nA}$. (c) Spatially resolved ST spectrum along the arrow in (a). (d) Spatially averaged ST spectrum over the region in (a). (b) Spatially resolved apparent barrier height for the region in (a). Figure originally published in [157]. Reprinted with permission from the American Physical Society. (C) (2019) American Physical Society. 



\section{Bibliography}

[1] Takase, A., Kojima, K., Komatsubara, T., and Kasuya, T., "Electrical resistivity and magnetoresistance of $\mathrm{CeB}_{6}$ ", Solid State Communications 36, 461-464 (1980).

[2] Effantin, J. M., Rossat-Mignod, J., Burlet, P., Bartholin, H., Kunii, S., and Kasuya, T., "Magnetic phase diagram of $\mathrm{CeB}_{6}$ ", Journal of Magnetism and Magnetic Materials 47-48, 145-148 (1985).

[3] Cameron, A. S., Friemel, G., and Inosov, D. S., "Multipolar phases and magnetically hidden order: review of the heavy-fermion compound $\mathrm{Ce}_{1-x} \mathrm{La}_{x} \mathrm{~B}_{6}$ ", Reports on Progress in Physics 79, 066502 (2016).

[4] Matthias, B. T., Geballe, T. H., Andres, K., Corenzwit, E., Hull, G. W., and Maita, J. P., "Superconductivity and Antiferromagnetism in BoronRich Lattices”, Science 159, 530 (1968).

[5] Cooley, J. C., Aronson, M. C., Sarrao, J. L., and Fisk, Z., "High pressures and ferromagnetic order in EuB 6 ", Physical Review B 56, 14541-14546 (1997).

[6] Süllow, S., Prasad, I., Aronson, M. C., Sarrao, J. L., Fisk, Z., Hristova, D., Lacerda, A. H., Hundley, M. F., Vigliante, A., and Gibbs, D., "Structure and magnetic order of EuB 6 ", Physical Review B 57, 5860-5869 (1998).

[7] Dzero, M., Sun, K., Galitski, V., and Coleman, P., “Topological Kondo Insulators", Physical Review Letters 104, 106408 (2010).

[8] Rößler, S., Jang, T.-H., Kim, D.-J., Tjeng, L. H., Fisk, Z., Steglich, F., and Wirth, S., "Hybridization gap and Fano resonance in $\mathrm{SmB}_{6}$ ", Proceedings of the National Academy of Sciences 111, 4798-4802 (2014).

[9] Hlawenka, P., Siemensmeyer, K., Weschke, E., Varykhalov, A., SánchezBarriga, J., Shitsevalova, N. Y., Dukhnenko, A. V., Filipov, V. B., Gabáni, S., Flachbart, K., Rader, O., and Rienks, E. D. L., "Samarium hexaboride is a trivial surface conductor", Nature Communications $\mathbf{9}$, 517 (2018).

[10] Herrmann, H., Hlawenka, P., Siemensmeyer, K., Weschke, E., SánchezBarriga, J., Varykhalov, A., Shitsevalova, N. Y., Dukhnenko, A. V., Filipov, V. B., Gabáni, S., Flachbart, K., Rader, O., Sterrer, M., and Rienks, E. D. L., "Contrast Reversal in Scanning Tunneling Microscopy and Its Implications for the Topological Classification of $\mathrm{SmB}_{6}$ ", Advanced Materials 32, 1906725 (2020). 
[11] Wirth, S., Rößler, S., Jiao, L., Crivillero, M. V. A., Rosa, P. F. S., and Fisk, Z., "Comparative Scanning Tunneling Microscopy Study on Hexaborides", physica status solidi (b), 2000022 (2020).

[12] Xia, M., Jiang, J., Ye, Z. R., Wang, Y. H., Zhang, Y., Chen, S. D., Niu, X. H., Xu, D. F., Chen, F., Chen, X. H., Xie, B. P., Zhang, T., and Feng, D. L., "Angle-resolved Photoemission Spectroscopy Study on the Surface States of the Correlated Topological Insulator $\mathrm{YbB}_{6}$ ", Scientific Reports 4, 5999 (2014).

[13] Neupane, M., Xu, S.-Y., Alidoust, N., Bian, G., Kim, D. J., Liu, C., Belopolski, I., Chang, T.-R., Jeng, H.-T., Durakiewicz, T., Lin, H., Bansil, A., Fisk, Z., and Hasan, M. Z., "Non-Kondo-like Electronic Structure in the Correlated Rare-Earth Hexaboride $\mathrm{YbB}_{6}$ ", Physical Review Letters 114, 016403 (2015).

[14] Kang, C.-J., Denlinger, J. D., Allen, J. W., Min, C.-H., Reinert, F., Kang, B. Y., Cho, B. K., Kang, J.-S., Shim, J. H., and Min, B. I., "Electronic Structure of $\mathrm{YbB}_{6}$ : Is it a Topological Insulator or Not?", Physical Review Letters 116, 116401 (2016).

[15] Lafferty, J. M., "Boride Cathodes", Journal of Applied Physics 22, 299309 (1951).

[16] Ahmed, H. and Broers, A. N., "Lanthanum Hexaboride Electron Emitter", Journal of Applied Physics 43, 2185-2192 (1972).

[17] Swanson, L. W. and Dickinson, T., "Single-crystal work-function and evaporation measurements of $\mathrm{LaB}_{6}$ ", Applied Physics Letters 28, 578580 (1976).

[18] Oshima, C., Bannai, E., Tanaka, T., and Kawai, S., "Thermionic work function of $\mathrm{LaB}_{6}$ single crystals and their surfaces", Journal of Applied Physics 48, 3925-3927 (1977).

[19] Nishitani, R., Aono, M., Tanaka, T., Oshima, C., Kawai, S., Iwasaki, H., and Nakamura, S., "Surface structures and work functions of the $\mathrm{LaB}_{6}$ (100), (110) and (111) clean surfaces”, Surface Science 93, 535-549 (1980).

[20] Broers, A. N., "Electron Gun using Long-Life Lanthanum Hexaboride Cathode", Journal of Applied Physics 38, 1991-1992 (1967).

[21] Broers, A. N., "Some experimental and estimated characteristics of the lanthanum hexaboride rod cathode electron gun", Journal of Physics E: Scientific Instruments 2, 273-276 (1969).

[22] Nakamoto, M. and Fukuda, K., "Field electron emission from $\mathrm{LaB}_{6}$ and TiN emitter arrays fabricated by transfer mold technique", Applied Surface Science 202, 289-294 (2002).

[23] Zhang, H., Tang, J., Zhang, Q., Zhao, G., Yang, G., Zhang, J., Zhou, O., and Qin, L.-C., "Field Emission of Electrons from Single $\mathrm{LaB}_{6}$ Nanowires", Advanced Materials 18, 87-91 (2005). 
[24] Zhang, H., Zhang, Q., Tang, J., and Qin, L.-C., "Single-Crystalline $\mathrm{LaB}_{6}$ Nanowires", Journal of the American Chemical Society 127, 2862-2863 (2005).

[25] Zhang, H., Zhang, Q., Zhao, G., Tang, J., Zhou, O., and Qin, L.-C., "Single-Crystalline $\mathrm{GdB}_{6}$ Nanowire Field Emitters", Journal of the American Chemical Society 127, 13120-13121 (2005).

[26] Takeda, H., Kuno, H., and Adachi, K., "Solar Control Dispersions and Coatings With Rare-Earth Hexaboride Nanoparticles", Journal of the American Ceramic Society 91, 2897-2902 (2008).

[27] Mattox, T. M. and Urban, J. J., "Tuning the Surface Plasmon Resonance of Lanthanum Hexaboride to Absorb Solar Heat: A Review", Materials 11, 2473 (2018).

[28] Mitterer, C., Komenda-Stallmaier, J., Losbichler, P., Schmölz, P., and Störi, H., "Decorative boride coatings based on $\mathrm{LaB}_{6}$ ", Surface and Coatings Technology, Fourth International Conference on Plasma Surface Engineering Part 2 74-75, 1020-1027 (1995).

[29] Mitterer, C., "Borides in Thin Film Technology", Journal of Solid State Chemistry 133, 279-291 (1997).

[30] Patil, S., Adhikary, G., Balakrishnan, G., and Maiti, K., "Unusual spectral renormalization in hexaborides", Journal of Physics: Condensed Matter 23, 495601 (2011).

[31] Schmidt, K., Jaime, O., Cahill, J., Edwards, D., Misture, S., Graeve, O., and Vasquez, V., "Surface termination analysis of stoichiometric metal hexaborides: Insights from first-principles and XPS measurements", Acta Materialia 144, 187-201 (2018).

[32] Ozcomert, J. S. and Trenary, M., "Atomically resolved surface structure of $\mathrm{LaB}_{6}(100)$ ", Surface Science 265, L227-L232 (1992).

[33] Ozcomert, J. S. and Trenary, M., "Atomic structure of the $\mathrm{LaB}_{6}(100)$ surface as observed with scanning tunneling microscopy", Journal of Vacuum Science \& Technology A 10, 2581-2584 (1992).

[34] Sotoudeh, M., Rajpurohit, S., Blöchl, P., Mierwaldt, D., Norpoth, J., Roddatis, V., Mildner, S., Kressdorf, B., Ifland, B., and Jooss, C., "Electronic structure of $\operatorname{Pr}_{1-x} \mathrm{Ca}_{x} \mathrm{MnO}_{3}$ ", Physical Review B 95, 235150 (2017).

[35] Krishnamurthy, N., Extractive Metallurgy of Rare Earths (CRC Press, 2004).

[36] Cardarelli, F., Materials Handbook: A Concise Desktop Reference (Springer London, 2008).

[37] Greenwood, N. N. and Earnshaw, A., Chemie der Elemente, 1st corrected reprint of the 1 st edition ( $\mathrm{VCH}, 1990)$.

[38] Muetterties, E. L., The chemistry of boron and its compounds (Wiley, 1967). 
[39] Stackelberg, M. v. and Neumann, F., "Die Kristallstruktur der Boride der Zusammensetzung $\mathrm{MeB}_{6}$ ", Zeitschrift für Physikalische Chemie 19B, 314-320 (1932).

[40] Samsonov, G. V., Paderno, Y. B., and Fomenko, V. S., "Hexaborides of the rare-earth metals", Soviet Powder Metallurgy and Metal Ceramics 2, 449-454 (1963).

[41] Mackinnon, I. D. R., Alarco, J. A., and Talbot, P. C., "Metal Hexaborides with Sc, Ti or Mn", Modeling and Numerical Simulation of Material Science 3, 158-169 (2013).

[42] Etourneau, J. and Hagenmuller, P., "Structure and physical features of the rare-earth borides", Philosophical Magazine Part B 52, 589-610 (1985).

[43] Perkins, P. G., "The Electronic Structures of the Hexaborides and the Diborides", in Boron and Refractory Borides (Springer-Verlag, Berlin, Heidelberg, New York, 1977).

[44] Gallagher, H. E., "Poisoning of $\mathrm{LaB}_{6}$ Cathodes", Journal of Applied Physics 40, 44-51 (1969).

[45] Goldstein, B. and Szostak, D. J., "Characterization of clean and oxidized (100) $\mathrm{LaB}_{6}$ ”, Surface Science 74, 461-478 (1978).

[46] Swanson, L. W. and McNeely, D. R., "Work functions of the (001) face of the hexaborides of Ba, La, Ce and Sm", Surface Science 83, 11-28 (1979).

[47] Chambers, S. A. and Swanson, L. W., "Angle-resolved Auger electron emission from $\mathrm{LaB}_{6}(001)$ with and without chemisorbed oxygen", Surface Science 131, 385-402 (1983).

[48] Futamoto, M., Nakazawa, M., and Kawabe, U., "High temperature surface composition of hexaboride thermionic electron emitters", Vacuum 33, 727-732 (1983).

[49] Aono, M., Nishitani, R., Oshima, C., Tanaka, T., Bannai, E., and Kawai, S., " $\mathrm{LaB}_{6}$ and $\mathrm{SmB}_{6}(001)$ surfaces studied by angle-resolved XPS, LEED and ISS", Surface Science, Proceedings of the International Conference on Solid Films and Surfaces Tokyo, Japan, 5-8 July 1978 86, 631-637 (1979).

[50] Aono, M., Tanaka, T., Bannai, E., Oshima, C., and Kawai, S., "Surface states of $\mathrm{LaB}_{6}(001)$ as revealed by angular-resolved ultraviolet photoelectron spectroscopy", Physical Review B 16, 3489-3492 (1977).

[51] Monnier, R. and Delley, B., "Properties of $\mathrm{LaB}_{6}$ elucidated by density functional theory”, Physical Review B 70, 193403 (2004).

[52] Uijttewaal, M. A., Wijs, G. A. de, and Groot, R. A. de, "Ab Initio and Work Function and Surface Energy Anisotropy of LaB6", The Journal of Physical Chemistry B 110, 18459-18465 (2006).

[53] Born, M. and Oppenheimer, R., "Zur Quantentheorie der Molekeln", Annalen der Physik 389, 457-484 (1927). 
[54] Max Born, Kun Huang, Dynamical Theory of Crystal Lattices (Clarendon Press, Oxford, 1968).

[55] Martin, R. M., Electronic Structure: basic theory and practical methods (Cambridge University Press, Cambridge, UK, 2004).

[56] Blöchl, P. E., ФSX: Advanced Topics of Theoretical Physics I: Introduction to Solid-State Theory (Lecture Notes) (Clausthal-Zellerfeld, 2020), http: //www2 . pt . tu-clausthal .de/atp/phisx.html (visited on 10/04/2020).

[57] Car, R. and Parrinello, M., "Unified Approach for Molecular Dynamics and Density-Functional Theory", Physical Review Letters 55, 24712474 (1985).

[58] Anderson, P. W., "Localized Magnetic States in Metals", Physical Review 124, 41-53 (1961).

[59] Czycholl, G., Theoretische Festkörperphysik: Von den klassischen Modellen zu modernen Forschungsthemen, 3rd, updated edition (SpringerVerlag, Berlin, Heidelberg, 2008).

[60] Hohenberg, P. and Kohn, W., "Inhomogeneous Electron Gas", Physical Review 136, B864-B871 (1964).

[61] Eschrig, H., The Fundamentals of Density Functional Theory, Vol. 32, Teubner-Texte zur Physik (Teubner, Stuttgart, Leipzig, 1996).

[62] Lieb, E. H., "Density functionals for coulomb systems", International Journal of Quantum Chemistry 24, 243-277 (1983).

[63] Gilbert, T. L., "Hohenberg-Kohn theorem for nonlocal external potentials", Physical Review B 12, 2111-2120 (1975).

[64] Harriman, J. E., "Orthonormal orbitals for the representation of an arbitrary density”, Physical Review A 24, 680-682 (1981).

[65] Percus, J. K., "The role of model systems in the few-body reduction of the N-fermion problem", International Journal of Quantum Chemistry 13, 89-124 (1978).

[66] Levy, M., "Universal variational functionals of electron densities, firstorder density matrices, and natural spin-orbitals and solution of the v-representability problem", Proceedings of the National Academy of Sciences 76, 6062-6065 (1979).

[67] Kohn, W. and Sham, L. J., "Self-Consistent Equations Including Exchange and Correlation Effects", Physical Review 140, A1133-A1138 (1965).

[68] Dreizler, R. M. and Gross, E. K. U., Density Functional Theory: An Approach to the Quantum Many-Body Problem (Springer-Verlag, Berlin, Heidelberg, 1990).

[69] Parr, R. G. and Yang, W., Density-Functional Theory of Atoms and Molecules (Oxford University Press, Oxford, New York, 1989). 
[70] Harris, J., "Adiabatic-connection approach to Kohn-Sham theory”, Physical Review A 29, 1648-1659 (1984).

[71] Perdew, J. P. and Zunger, A., "Self-interaction correction to densityfunctional approximations for many-electron systems", Physical Review B 23, 5048-5079 (1981).

[72] Barth, U. v. and Hedin, L., "A local exchange-correlation potential for the spin polarized case. i", Journal of Physics C: Solid State Physics 5, 1629-1642 (1972).

[73] Rajagopal, A. K. and Callaway, J., "Inhomogeneous Electron Gas", Physical Review B 7, 1912-1919 (1973).

[74] Janak, J. F., "Proof that $\partial E / \partial n_{i}=\varepsilon_{i}$ in density-functional theory", Physical Review B 18, 7165-7168 (1978).

[75] Dirac, P. A. M., "Note on Exchange Phenomena in the Thomas Atom", Mathematical Proceedings of the Cambridge Philosophical Society 26, 376-385 (1930).

[76] Ceperley, D. M. and Alder, B. J., "Ground State of the Electron Gas by a Stochastic Method”, Physical Review Letters 45, 566-569 (1980).

[77] Vosko, S. H., Wilk, L., and Nusair, M., "Accurate spin-dependent electron liquid correlation energies for local spin density calculations: a critical analysis", Canadian Journal of Physics 58, 1200-1211 (1980).

[78] Perdew, J. P. and Wang, Y., "Accurate and simple analytic representation of the electron-gas correlation energy", Physical Review B 45, 1324413249 (1992).

[79] Perdew, J. P. and Wang, Y., "Erratum: Accurate and simple analytic representation of the electron-gas correlation energy [Phys. Rev. B 45, 13244 (1992)]", Physical Review B 98, 079904 (2018).

[80] Gunnarsson, O. and Lundqvist, B. I., "Exchange and correlation in atoms, molecules, and solids by the spin-density-functional formalism", Physical Review B 13, 4274-4298 (1976).

[81] Gunnarsson, O., Jonson, M., and Lundqvist, B. I., "Descriptions of exchange and correlation effects in inhomogeneous electron systems", Physical Review B 20, 3136-3164 (1979).

[82] Jones, R. O. and Gunnarsson, O., "The density functional formalism, its applications and prospects", Reviews of Modern Physics 61, 689-746 (1989).

[83] Perdew, J. P. and Kurth, S., "Density Functionals for Non-relativistic Coulomb Systems in the New Century", in A Primer in Density Functional Theory, edited by Fiolhais, C., Nogueira, F., and Marques, M. A. L., Lecture Notes in Physics (Springer-Verlag, Berlin, Heidelberg, 2003).

[84] Ernzerhof, M., Perdew, J. P., and Burke, K., "Coupling-constant dependence of atomization energies", International Journal of Quantum Chemistry 64, 285-295 (1997). 
[85] Becke, A. D., "Density-functional exchange-energy approximation with correct asymptotic behavior", Physical Review A 38, 3098-3100 (1988).

[86] Perdew, J. P., Burke, K., and Ernzerhof, M., "Generalized Gradient Approximation Made Simple”, Physical Review Letters 77, 3865-3868 (1996).

[87] Sawatzky, G. A. and Allen, J. W., "Magnitude and Origin of the Band Gap in NiO”, Physical Review Letters 53, 2339-2342 (1984).

[88] Terakura, K., Oguchi, T., Williams, A. R., and Kübler, J., "Band theory of insulating transition-metal monoxides: Band-structure calculations", Physical Review B 30, 4734-4747 (1984).

[89] Anisimov, V. I., Solovyev, I. V., Korotin, M. A., Czyżyk, M. T., and Sawatzky, G. A., "Density-functional theory and NiO photoemission spectra”, Physical Review B 48, 16929-16934 (1993).

[90] Perdew, J. P., Parr, R. G., Levy, M., and Balduz, J. L., "DensityFunctional Theory for Fractional Particle Number: Derivative Discontinuities of the Energy", Physical Review Letters 49, 1691-1694 (1982).

[91] Sham, L. J. and Schlüter, M., "Density-Functional Theory of the Energy Gap", Physical Review Letters 51, 1888-1891 (1983).

[92] Perdew, J. P. and Levy, M., "Physical Content of the Exact Kohn-Sham Orbital Energies: Band Gaps and Derivative Discontinuities", Physical Review Letters 51, 1884-1887 (1983).

[93] Gunnarsson, O. and Schönhammer, K., "Density-Functional Treatment of an Exactly Solvable Semiconductor Model", Physical Review Letters 56, 1968-1971 (1986).

[94] Svane, A. and Gunnarsson, O., "Transition-metal oxides in the selfinteraction-corrected density-functional formalism", Physical Review Letters 65, 1148-1151 (1990).

[95] Anisimov, V. I., Zaanen, J., and Andersen, O. K., "Band theory and Mott insulators: Hubbard $U$ instead of Stoner I", Physical Review B 44, 943-954 (1991).

[96] Anisimov, V. I., Aryasetiawan, F., and Lichtenstein, A. I., "First-principles calculations of the electronic structure and spectra of strongly correlated systems: the LDA $+U$ method", Journal of Physics: Condensed Matter 9, 767-808 (1997).

[97] Metzner, W. and Vollhardt, D., "Correlated Lattice Fermions in $d=\infty$ Dimensions”, Physical Review Letters 62, 324-327 (1989).

[98] Georges, A., Kotliar, G., Krauth, W., and Rozenberg, M. J., "Dynamical mean-field theory of strongly correlated fermion systems and the limit of infinite dimensions", Reviews of Modern Physics 68, 13-125 (1996). 
[99] Lechermann, F., Georges, A., Poteryaev, A., Biermann, S., Posternak, M., Yamasaki, A., and Andersen, O. K., "Dynamical mean-field theory using Wannier functions: A flexible route to electronic structure calculations of strongly correlated materials", Physical Review B 74, 125120 (2006).

[100] Amadon, B., Lechermann, F., Georges, A., Jollet, F., Wehling, T. O., and Lichtenstein, A. I., "Plane-wave based electronic structure calculations for correlated materials using dynamical mean-field theory and projected local orbitals", Physical Review B 77, 205112 (2008).

[101] Aichhorn, M., Pourovskii, L., Vildosola, V., Ferrero, M., Parcollet, O., Miyake, T., Georges, A., and Biermann, S., "Dynamical meanfield theory within an augmented plane-wave framework: Assessing electronic correlations in the iron pnictide LaFeAsO", Physical Review B 80, 085101 (2009).

[102] Becke, A. D., "A new mixing of Hartree-Fock and local densityfunctional theories", The Journal of Chemical Physics 98, 1372-1377 (1993).

[103] Becke, A. D., "Density-functional thermochemistry. III. The role of exact exchange", The Journal of Chemical Physics 98, 5648-5652 (1993).

[104] Stephens, P. J., Devlin, F. J., Chabalowski, C. F., and Frisch, M. J., "Ab Initio Calculation of Vibrational Absorption and Circular Dichroism Spectra Using Density Functional Force Fields", The Journal of Physical Chemistry 98, 11623-11627 (1994).

[105] Lee, C., Yang, W., and Parr, R. G., "Development of the Colle-Salvetti correlation-energy formula into a functional of the electron density", Physical Review B 37, 785-789 (1988).

[106] Becke, A. D., "Density-functional thermochemistry. IV. A new dynamical correlation functional and implications for exact-exchange mixing", The Journal of Chemical Physics 104, 1040-1046 (1996).

[107] Perdew, J. P., Ernzerhof, M., and Burke, K., "Rationale for mixing exact exchange with density functional approximations", The Journal of Chemical Physics 105, 9982-9985 (1996).

[108] Adamo, C. and Barone, V., "Toward reliable density functional methods without adjustable parameters: The PBE0 model", The Journal of Chemical Physics 110, 6158-6170 (1999).

[109] Ernzerhof, M. and Scuseria, G. E., "Assessment of the Perdew-BurkeErnzerhof exchange-correlation functional", The Journal of Chemical Physics 110, 5029-5036 (1999).

[110] Jochen Heyd and Gustavo E. Scuseria, "Efficient hybrid density functional calculations in solids: Assessment of the Heyd-Scuseria-Ernzerhof screened Coulomb hybrid functional", The Journal of Chemical Physics 121, 1187-1192 (2004). 
[111] Paier, J., Marsman, M., Hummer, K., Kresse, G., Gerber, I. C., and Ángyán, J. G., "Screened hybrid density functionals applied to solids", The Journal of Chemical Physics 124, 154709 (2006).

[112] Jochen Heyd, Gustavo E. Scuseria, and Matthias Ernzerhof, "Hybrid functionals based on a screened Coulomb potential", The Journal of Chemical Physics 118, 8207-8215 (2003).

[113] Jochen Heyd, Gustavo E. Scuseria, and Matthias Ernzerhof, "Erratum: "Hybrid functionals based on a screened Coulomb potential" [J. Chem. Phys. 118, 8207 (2003)]", The Journal of Chemical Physics 124, 219906 (2006).

[114] Jaramillo, J., Scuseria, G. E., and Ernzerhof, M., "Local hybrid functionals", The Journal of Chemical Physics 118, 1068-1073 (2003).

[115] Maier, T. M., Arbuznikov, A. V., and Kaupp, M., "Local hybrid functionals: Theory, implementation, and performance of an emerging new tool in quantum chemistry and beyond", WIREs Computational Molecular Science 9, e1378 (2019).

[116] Blöchl, P. E., Private Communication, 2018.

[117] Varshalovich, D. A., Moskalev, A. N., and Khersonskiı̌, V. K., Quantum Theory of Angular Momentum: Irreducible Tensors, Spherical Harmonics, Vector Coupling Coefficients, 3nj Symbols (World Scientific Pub., 1988).

[118] Blöchl, P. E., Walther, C. F. J., and Pruschke, T., "Method to include explicit correlations into density-functional calculations based on densitymatrix functional theory”, Physical Review B 84, 205101 (2011).

[119] Blöchl, P. E., Manual for the Projector Augmented Wave Method (included with the CP-PAW simulation code) (Sept. 2018).

[120] Blöchl, P. E., "Projector augmented-wave method", Physical Review B 50, 17953-17979 (1994).

[121] Blöchl, P. E., Först, C. J., and Schimpl, J., "The Projector Augmented Wave Method: ab-initio molecular dynamics with full wave functions", arXiv:cond-mat/0201015 (2002).

[122] Binnig, G., Rohrer, H., Gerber, C., and Weibel, E., "Surface Studies by Scanning Tunneling Microscopy", Physical Review Letters 49, 57-61 (1982).

[123] Binnig, G., Rohrer, H., Gerber, C., and Weibel, E., “7×7 Reconstruction on Si(111) Resolved in Real Space", Physical Review Letters 50, 120 123 (1983).

[124] Chen, C. J., Introduction to Scanning Tunneling Microscopy (Oxford University Press, New York, May 20, 1993).

[125] Bardeen, J., “Tunnelling from a Many-Particle Point of View”, Physical Review Letters 6, 57-59 (1961). 
[126] Tersoff, J. and Hamann, D. R., "Theory and Application for the Scanning Tunneling Microscope", Physical Review Letters 50, 1998-2001 (1983).

[127] Tersoff, J. and Hamann, D. R., "Theory of the scanning tunneling microscope", Physical Review B 31, 805-813 (1985).

[128] Lounis, S., “Theory of Scanning Tunneling Microscopy”, arXiv:1404.0961 [cond-mat] (2014).

[129] Chen, C. J., "Tunneling matrix elements in three-dimensional space: The derivative rule and the sum rule", Physical Review B 42, 88418857 (1990).

[130] Blöchl, P. E., ФSX: Quantum Mechanics of the Chemical Bond (Lecture Notes) (Clausthal-Zellerfeld, 2020), http : / / www2 . pt . tu clausthal.de/atp/phisx.html (visited on 10/04/2020).

[131] Tinkham, M., Group Theory and Quantum Mechanics, Dover Books on Chemistry (Dover Publications, 2012).

[132] Longuet-Higgins, H. C. and Roberts, M. d. V., "The electronic structure of the borides $M B_{6}$ ", Proc. R. Soc. Lond. A 224, 336-347 (1954).

[133] Singh, N., Saini, S. M., Nautiyal, T., and Auluck, S., "Electronic structure and optical properties of rare earth hexaborides $\mathrm{RB}_{6}(\mathrm{R}=\mathrm{La}, \mathrm{Ce}$, Pr, Nd, Sm, Eu, Gd)", Journal of Physics: Condensed Matter 19, 346226 (2007).

[134] Harima, H., Sakai, O., Kasuya, T., and Yanase, A., "New interpretation of the de Haas-van Alphen signals of $\mathrm{LaB}_{6}$ ", Solid State Communications 66, 603-607 (1988).

[135] Kimura, S., Nanba, T., Tomikawa, M., Kunii, S., and Kasuya, T., "Electronic structure of rare-earth hexaborides", Physical Review B 46, 12196-12204 (1992).

[136] Shino, N., Suga, S., Imada, S., Saitoh, Y., Yamada, H., Nanba, T., Kimura, S., and Kunii, S., "Photoemission, Inverse Photoemission and Optical Studies of Rare-Earth Hexaborides", Journal of the Physical Society of Japan 64, 2980-2987 (1995).

[137] Ōnuki, Y. and Hasegawa, A., "Fermi Surfaces of Intermetallic Compounds", in Handbook on the Physics and Chemistry of Rare Earths, Vol. 20, edited by Karl A. Gschneidner, Jr. and Eyring, L. (Elsevier, Amsterdam, 1995).

[138] Tanaka, K. and Ōnuki, Y., “Observation of 4 f electron transfer from Ce to $\mathrm{B}_{6}$ in the Kondo crystal $\mathrm{CeB}_{6}$ and its mechanism by multi-temperature X-ray diffraction", Acta Crystallographica Section B: Structural Science 58, 423-436 (2002).

[139] Blomberg, M. K., Merisalo, M. J., Korsukova, M. M., and Gurin, V. N., "Single-crystal X-ray diffraction study of $\mathrm{NdB}_{6}, \mathrm{EuB}_{6}$ and $\mathrm{YbB}_{6}$ ", Journal of Alloys and Compounds 217, 123-127 (1995). 
[140] Neupane, M., Alidoust, N., Belopolski, I., Bian, G., Xu, S.-Y., Kim, D.-J., Shibayev, P. P., Sanchez, D. S., Zheng, H., Chang, T.-R., Jeng, H.-T., Riseborough, P. S., Lin, H., Bansil, A., Durakiewicz, T., Fisk, Z., and Hasan, M. Z., "Fermi surface topology and hot spot distribution in the Kondo lattice system CeB 6 ”, Physical Review B 92, 104420 (2015).

[141] Chazalviel, J. N., Campagna, M., Wertheim, G. K., Schmidt, P. H., and Yafet, Y., "Electronic Structure and $4 f$-Hole Lifetime in Rare-Earth Borides", Physical Review Letters 37, 919-922 (1976).

[142] Speier, W., Zeller, R., and Fuggle, J. C., "Studies of total density of states of metals up to $70 \mathrm{eV}$ above $E_{\mathrm{F}}$ ", Physical Review B 32, 35973603 (1985).

[143] Aryasetiawan, F. and Gunnarsson, O., "The GW method", Reports on Progress in Physics 61, 237 (1998).

[144] Jaynes, E. T., "Information Theory and Statistical Mechanics", Physical Review 106, 620-630 (1957).

[145] Jaynes, E. T., "Information Theory and Statistical Mechanics. II", Physical Review 108, 171-190 (1957).

[146] Sethna, J. P., Statistical Mechanics: Entropy, Order Parameters, and Complexity, Oxford Master Series in Physics 14 (Oxford University Press, Oxford, New York, 2012).

[147] Kardar, M., Statistical Physics of Particles (Cambridge University Press, Cambridge, UK, 2017).

[148] Vainshtein, V. D. and Gol'fand, Y. A., "Quantum Measurements and the Theory of Macroscopic Processes", in Quantum Mechanics and Statistical Methods, edited by Sushchinskiy, M. M., red. by Basov, N. G., Proceedings of the Lebedev Physics Institute of the Academy of Sciences of the USSR 173 (Nova Science Publishers, Commack, New York, 1988).

[149] Kanamori, J., "Electron Correlation and Ferromagnetism of Transition Metals", Progress of Theoretical Physics 30, 275-289 (1963).

[150] Georges, A., Medici, L. de', and Mravlje, J., "Strong Correlations from Hund's Coupling", Annual Review of Condensed Matter Physics 4, 137-178 (2013).

[151] Czyżyk, M. T. and Sawatzky, G. A., "Local-density functional and onsite correlations: The electronic structure of $\mathrm{La}_{2} \mathrm{CuO}_{4}$ and $\mathrm{LaCuO}_{3}$ ", Physical Review B 49, 14211-14228 (1994).

[152] Petukhov, A. G., Mazin, I. I., Chioncel, L., and Lichtenstein, A. I., "Correlated metals and the LDA $+U$ method", Physical Review B 67, 153106 (2003).

[153] Himmetoglu, B., Floris, A., Gironcoli, S. d., and Cococcioni, M., "Hubbard-corrected DFT energy functionals: The LDA+U description of correlated systems", International Journal of Quantum Chemistry 114, 14-49 (2014). 
[154] Gull, E., Millis, A. J., Lichtenstein, A. I., Rubtsov, A. N., Troyer, M., and Werner, P., "Continuous-time Monte Carlo methods for quantum impurity models", Reviews of Modern Physics 83, 349-404 (2011).

[155] Shinaoka, H., Gull, E., and Werner, P., "Continuous-time hybridization expansion quantum impurity solver for multi-orbital systems with complex hybridizations", Computer Physics Communications 215, 128-136 (2017).

[156] Jarrell, M. and Gubernatis, J. E., "Bayesian inference and the analytic continuation of imaginary-time quantum Monte Carlo data", Physics Reports 269, 133-195 (1996).

[157] Buchsteiner, P., Sohn, F., Horstmann, J. G., Voigt, J., Ciomaga Hatnean, M., Balakrishnan, G., Ropers, C., Blöchl, P. E., and Wenderoth, M., "Surface resonance of the $(2 \times 1)$ reconstructed lanthanum hexaboride (001)-cleavage plane: A combined STM and DFT study", Physical Review B 100, 205407 (2019).

[158] Balakrishnan, G., Lees, M. R., and Paul, D. McK., "Growth of large single crystals of rare earth hexaborides", Journal of Crystal Growth 256, 206-209 (2003).

[159] Balakrishnan, G., Lees, M. R., and Paul, D. M'K., "Rare earth hexaborides: large single crystals", Journal of Magnetism and Magnetic Materials, Proceedings of the International Conference on Magnetism (ICM 2003) 272-276, 601-602 (2004).

[160] Vogelgesang, S., Storeck, G., Horstmann, J. G., Diekmann, T., Sivis, M., Schramm, S., Rossnagel, K., Schäfer, S., and Ropers, C., "Phase ordering of charge density waves traced by ultrafast low-energy electron diffraction", Nature Physics 14, 184-190 (2018).

[161] Rößler, S., Jiao, L., Kim, D. J., Seiro, S., Rasim, K., Steglich, F., Tjeng, L. H., Fisk, Z., and Wirth, S., "Surface and electronic structure of $\mathrm{SmB}_{6}$ through scanning tunneling microscopy", Philosophical Magazine 96, 3262-3273 (2016).

[162] Wick, G. C., "The Evaluation of the Collision Matrix", Physical Review 80, 268-272 (1950).

[163] Ring, P. and Schuck, P., The nuclear many-body problem (SpringerVerlag, New York, Heidelberg, Berlin, 1980).

[164] Fetter, A. L. and Walecka, J. D., Quantum Theory of Many-Particle Systems (Dover Publications, Mineola, New York, 2003).

[165] Garleff, J. K., Wenderoth, M., Sauthoff, K., Ulbrich, R. G., and Rohlfing, M., " $(2 \times 1)$ reconstructed $\mathrm{Si}(111)$ surface: STM experiments versus $a b$ initio calculations”, Physical Review B 70, 245424 (2004). 


\section{Acknowledgments}

First of all I would like to express my gratitude to Thomas Pruschke (†2016), who kindly gave me the opportunity to work in his group and to do the research in the context of this thesis. Even under his most dire health conditions he was ready and happy to give advice in his most memorable friendly way. He stayed involved in the research projects of his students and continued to support me and the other students of his group, even when he was in hospital.

I very much thank Peter Blöchl, who, after co-supervising me in the beginning of the $\mathrm{PhD}$, took over responsibility as my main supervisor. After Thomas's sad demise, Peter took me into his group and made it possible for me to continue my $\mathrm{PhD}$ project under his most appreciated and valuable guidance. Throughout my $\mathrm{PhD}$ he was always open for discussions and happy to give advice.

Many thanks also to Salvatore Manmana who took over responsibilities for me and Thomas's other students, and who stayed involved in my $\mathrm{PhD}$ project as my third supervisor. Especially his various helpful contributions in our discussions and also his help in administrative tasks, including applications for HPC access, are much appreciated.

I would very much like to thank Philipp Buchsteiner and Martin Wenderoth for many fruitful discussions and a great and close collaboration. Our meetings were always enjoyable and I gained many insights into the experimental work with the scanning tunneling microscope. Moreover, a special thanks goes to Martin, who became my second supervisor and took over administrative responsibilities concerning my $\mathrm{PhD}$, which is much appreciated.

I thank Stefan Kehrein for his willingness to co-referee my thesis and I thank Vasile Moshnyaga and Marcus Müller for being members of my thesis defense commission.

Thanks also go to Roser Valentí at the University of Frankfurt and to Emanuel Gull at the University of Michigan, Ann Arbor, MI, for their hospitality and for our fruitful discussions. A big thanks also to Steffen Backes for our instructive and enjoyable discussions on the various aspects of DFT+DMFT.

I very much thank all my past and present colleagues for the enjoyable and friendly atmosphere at the Institute for Theoretical Physics in Göttingen, and for the various opportunities for physics-related (and sometimes also non-physicsrelated discussions). Furthermore, I would like to mention that I had a great time during our leisure activities and will remember our coffee breaks, our group outings, our sushi dinners, our barbecues, and our Thursday evening dinners with subsequent bowling session.

For proofreading parts of my thesis and for giving valuable comments I thank my colleagues Michael ten Brink, Constantin Meyer, and Philipp Buchsteiner.

Moreover, I gratefully acknowledge financial support by the German Research Foundation, Grants No. BL539/10-1 and No. PR298/19-1. I also thank 
Bibliography

Jürgen Holm and the GWDG for their IT services and support.

Finally, big thanks to my family, especially my parents, who supported me through all the ups and downs of my $\mathrm{PhD}$ studies. 
This thesis has been typeset in IATEX.

Figures have been created using the following software:

- Inkscape, https://inkscape.org/

- GIMP, https://www.gimp.org/

- VESTA, http: //www.jp-minerals.org/vesta/en/

Momma, K. and Izumi, F., "VESTA 3 for three-dimensional visualization of crystal, volumetric and morphology data", J. Appl. Crystallogr., 44, 1272-1276 (2011)

- POV-ray, Persistence of Vision Pty. Ltd. (2004), http: //www . povray . org/

- Asymptote, https://asymptote. sourceforge.io/

- PGF/TikZ, https://github.com/pgf-tikz/pgf

- PGFPlots http://pgfplots.sourceforge.net/

- Matplotlib, https://matplotlib.org/ 


\section{Publication in the context of this thesis}

P. Buchsteiner, F. Sohn, J. G. Horstmann, J. Voigt, M. Ciomaga Hatnean, G. Balakrishnan, C. Ropers, P. E. Blöchl, and M. Wenderoth. "Surface resonance of the $(2 \times 1)$ reconstructed lanthanum hexaboride $(001)$-cleavage plane: A combined STM and DFT study", Physical Review B 100, 205407 (Nov 2019)

DoI: $10.1103 /$ PhysRevB . 100.205407

URL: https://journals.aps.org/prb/abstract/10.1103/

PhysRevB. 100.205407 